\title{
Life-Cycle Transformations of Metal and Metal Oxide Nanoparticles from Commercial Products
}

\author{
By
}

Selvan Mohan

A thesis submitted to the Faculty of Graduate and Postdoctoral Affairs in partial fulfillment of the requirements for the degree of

Doctor of Philosophy

in

Chemistry

Carleton University

Ottawa, Ontario

(C) 2019, Selvan Mohan 


\begin{abstract}
The environmental fate and effects of engineered nanomaterials have become a topic of interest to researchers due to their increased incorporation within consumer products. Current research into the effects of nanoparticles towards the environment focuses almost entirely on using the pristine form of the nanoparticles for testing. However, due to the countless evidence of physical and chemical transformations to which nanoparticles are often subjected, the appropriateness of using pristine nanoparticles for environmental testing is being questioned. Through this research, we examine the chemical and physical transformations of nanoparticles in commercial products by modeling typical use scenarios that could occur throughout the product lifecycle. Nanomaterial-containing commercial products were put through conditions mimicking a typical lifecycle from product incorporation to leaching into the wastewater collection stream. A combination of transmission electron microscopy (TEM), energy dispersive X-ray spectroscopy (EDS), X-ray photoelectron spectroscopy (XPS), and inductively coupled plasma-mass spectrometry (ICP-MS) was used to examine the morphological and compositional effects of the product lifecycle on the nanoparticles.
\end{abstract}

Silver nanoparticles (AgNPs) have seen widespread use in consumer products due to their exceptional antimicrobial properties. We studied the transformation of AgNPs from the production of AgNPs-laden textiles, through day-to-day use scenarios and leaching into the wastewater collection system. The day-to-day use includes exposure to sweat due to the market for the AgNP-containing sports clothing, as well as laundering, which would expose the nanoparticles to detergent and bleach. Exposure to these weathering conditions showed the aggregation and agglomeration of AgNPs as well as 
transformation into $\mathrm{AgCl}$ and $\mathrm{Ag}_{2} \mathrm{~S}$. Despite the transformations above, the nanoparticle form of $\mathrm{Ag}$ was still present after the various use conditions.

Zinc Oxide nanoparticles (ZnO NPs) have been widely used for their antimicrobial properties and, more recently as a UV blocker. The lifecycle of ZnO NPs was tested within the medicated baby powder, athletic socks, sunscreen, and UV protective textiles as well as in the pristine nanoparticle form. The nanoparticlecontaining products were weathered through day-to-day use, which included sweat, chlorinated water from swimming pools, and UV exposure, followed by laundering in detergent and bleach, and finally making its way into wastewater collection. ZnO NPs were observed to dissolve into solution, a process accelerated by an acidic environment. Transformation to $\mathrm{ZnCl}_{2}$ and $\mathrm{ZnS}$ salts were also observed through the various weathering processes.

Cerium oxide nanoparticles $\left(\mathrm{CeO}_{2} \mathrm{NPs}\right)$ have been gaining attention in recent years due to their remarkable catalytic antioxidant properties. Moreover, $\mathrm{CeO}_{2} \mathrm{NPs}_{\text {save }}$ the ability to block UV rays and are transparent in the visible spectrum. $\mathrm{CeO}_{2} \mathrm{NPs}$ have not been widely used in consumer products; however, due to the many benefits of $\mathrm{CeO}_{2}$ NPs, it had been suggested to be used as a UV blocker by various sources. Assuming the inevitable use of $\mathrm{CeO}_{2}$ nanoparticles in sunscreens, we have impregnated current consumer sunscreens with $\mathrm{CeO}_{2}$ NPs and analyzed the chemical and physical transformation through the lifecycle of the product. $\mathrm{CeO}_{2} \mathrm{NP}$ impregnated sunscreens, and pristine nanoparticles were weathered through day-to-day use scenarios, which included exposure to sweat, chlorinated pool water, and UV exposure followed by the leaching of nanoparticles into the wastewater collection system. The $\mathrm{CeO}_{2} \mathrm{NPs}$ were 
observed to agglomerate and chemically associate with chlorine and sulfur after these treatments.

Overall, this thesis provides data that can be used to develop a more relevant model "weathered nanoparticle" sample that can be used in place of pristine nanoparticles for environmental studies seeking to inform future regulatory decision making. 


\section{Preface}

This preface provides full bibliographical details for each article included in this thesis, as well as whether the article is reproduced in whole or in part. The use of copyrighted material is likewise acknowledged here. When citing material from this thesis, please cite the article relevant to the chapter, if the chapter is based on a publication.

This thesis is constructed in the form of an integrated thesis and it consists of a total of 6 chapters as follows:

Chapter 1 is a brief general introduction to the research topics, including research objectives, methodology, approach, a summary review of the literature that presents the relevant theoretical background information, as well as the previous works most related to the research, and a list of hypotheses for examination. The chapter is prepared for thesis purposes only.

Chapter 2 provides a general description of the analytical techniques used throughout this research. The chapter is prepared for thesis purposes only.

\section{Chapter 3}

Mohan, S.; Princz, J.; Ormeci, B.; DeRosa, M.C. Morphological Transformation of Silver Nanoparticles from Commercial Products: Modeling from Product Incorporation, Weathering through Use Scenarios, and Leaching into Wastewater. Nanomaterials 2019, 9, 1258. 
The article is wholly reproduced and edited for formatting and clarity of presentation. Selvan Mohan performed all the experimental work. This article was written by Selvan Mohan. Maria DeRosa assisted with result interpretation. Juliska Princz, Banu Ormeci, and Maria DeRosa assisted with the experimental design and editing of the article.

\section{Chapter 4}

Mohan, S.; Phillips, K.; Princz, J.; DeRosa, M.C. Transformation of Zinc Oxide nanoparticles during potential use scenarios from product incorporation to collection in the wastewater stream. (pending submission).

The article was written for publication purposes. Selvan Mohan performed the majority of experimental work with the assistance of Kelsey Philip on experimental and sample preparations. This article was written by Selvan Mohan. Maria DeRosa assisted with result interpretation and editing the article. Juliska Princz and Maria DeRosa assisted with the experimental design.

\section{Chapter 5}

Mohan, S.; Phillips, K.; Princz, J.; DeRosa, M.C. Transformation of Cerium Oxide nanoparticles during potential use scenarios from product incorporation to collection in the wastewater stream. (pending submission).

The article was written for publication purposes. Selvan Mohan performed the majority of experimental work with the assistance of Kelsey Philip on experimental and sample preparations. This article was written by Selvan Mohan. Maria DeRosa assisted 
with result interpretation and editing the article. Juliska Princz and Maria DeRosa assisted with the experimental design.

Chapter 6 provides the overall conclusions, application of the research, and approach of future work. The chapter is prepared for thesis purposes only.

Note: Since this thesis is in a paper-based format, it is important to note that some of the information in the introduction and materials and methods of the papers might be repeated.

Pursuant to the Integrated Thesis policy of Carleton University, the "supervisor" (Maria DeRosa) and the "student" (Selvan Mohan) confirm that the student was fully involved in setting up and conducting the research, obtaining data and analyzing results, as well as preparing and writing the material presented in the co-authored article(s) integrated in the thesis. Additionally, the supervisor confirms the information provided by the student in this preface. 


\section{Acknowledgement}

First and foremost, I want to thank my supervisor, Dr. Maria DeRosa, for being extremely patient and encouraging through my doctorate journey. Every meeting with her energizes me and provides me with new hope to keep trying until my experiments succeed. Her guidance through every step got me towards completing this thesis. She had not only taught me how to be a scientist but also had been a part of my personal development.

I would like to thank Dr. Juliska Princz at Environment Canada for her guidance and insight towards understanding the significance of my research. I would also like to thank Dr. Dina Schwertfeger and Jessica Velicogna at Environment Canada for training me and trusting me to use the various instruments at the Nano-Laboratory of Environment Canada.

I would like to thank Environment Canada and Carleton University for funding my research and me through my studies.

I would like to thank Dr. Jianqun Wang for the hundreds of hours spent together with the TEM and SEM. You had taught me so much about those instruments and had always been very entertaining during all the time we spend with a "not very" cooperative instrument.

Thank you to everyone at the DeRosa Lab for being extremely supportive of my research even though I was the only one who did not work with aptamers. I would like to especially thank Kelsey Philip, David Tong and Fuad Alami for being great undergraduate students. You guys dedicated countless evenings and weekends towards 
the success of various projects, some not part of this thesis. I would also like to thank the Chemistry department of Carleton University for providing such an amazing facility and countless resources that had helped in my academic career.

A very special thanks for the depths of my heart to my mom and dad, despite being in the literal opposite side of the world, had checked on me every day and helped me keep my sanity. Despite not understanding anything about my research, they would listen to my rants of every failed experiment and kept reassuring me of my capabilities.

Finally, Katrin, you had been by my side through my entire Ph.D., putting up with all the ups and downs that it had brought us. Couldn't have done this without you. Thank you! 


\section{Table of Content}

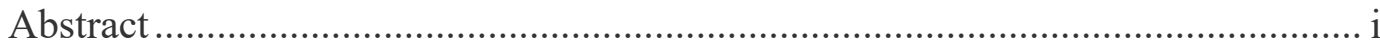

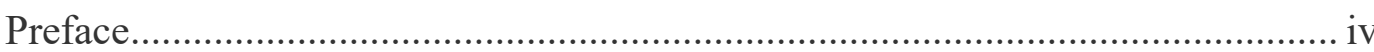

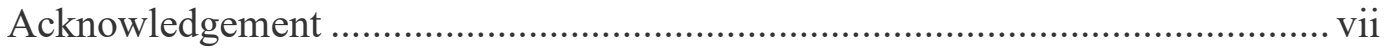

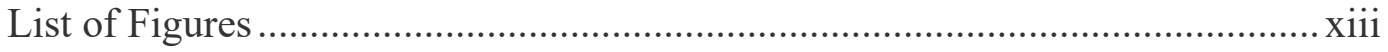

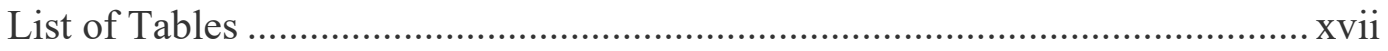

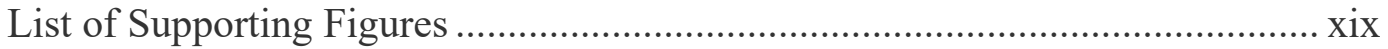

List of Supporting Tables........................................................................ xxii

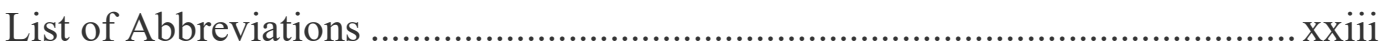

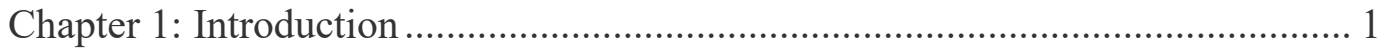

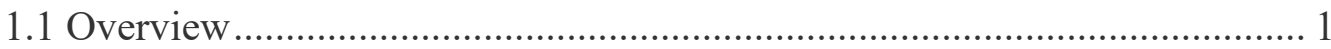

1.2 Objective of the study ..................................................................... 2

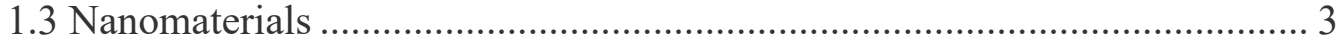

1.3.1 Current Standing on Nanoparticle Toxicity ..................................... 6

1.3.2 Nanoparticle Transformations ....................................................... 9

1.3.2.1 Morphological Transformation ................................................... 9

1.3.2.2 Compositional Transformation .............................................. 10

1.4 Nanoparticles Researched .................................................................. 10

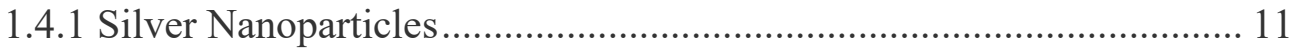

1.4.1.1 Nanoparticle Properties ........................................................ 11

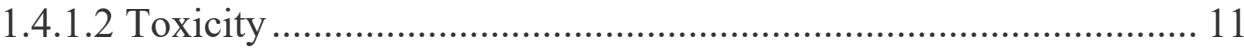

1.4.1.3 Known Transformations ........................................................ 13

1.4.2 Zinc Oxide Nanoparticles ............................................................ 14

1.4.2.1 Nanoparticle Properties ............................................................. 14

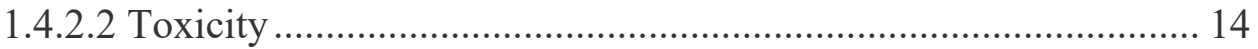

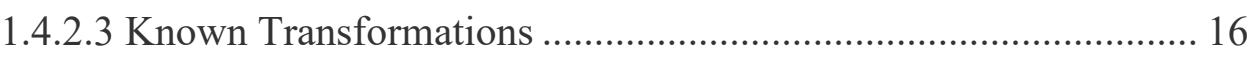

1.4.3 Cerium Oxide Nanoparticles........................................................... 16

1.4.3.1 Nanoparticle Properties................................................................ 16

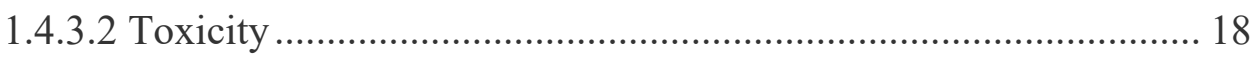

1.4.3.3 Known Transformations ........................................................ 18

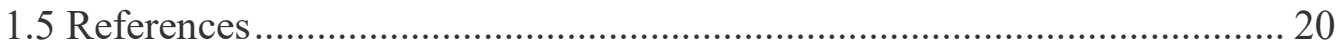


Chapter 2: Analysis Techniques ……………………....................................... 29

2.1 Transmission Electron Microscope (TEM) ……………………………..... 29

2.2 Energy-Dispersive X-ray Spectroscopy (EDS) ………………………..... 33

2.3 Inductively coupled plasma, mass spectrometry (ICP-MS) ........................ 35

2.4 X-Ray Photoelectron Spectrometer (XPS) ……………………………….. 38

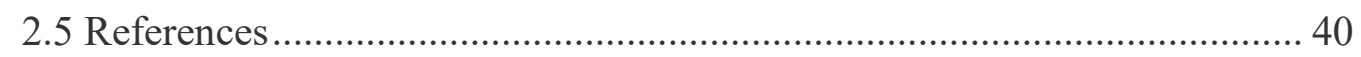

Chapter 3: Morphological transformation of silver nanoparticles from commercial products: modeling from product incorporation, weathering through use scenarios, and

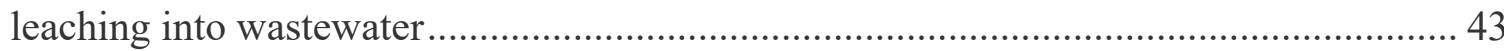

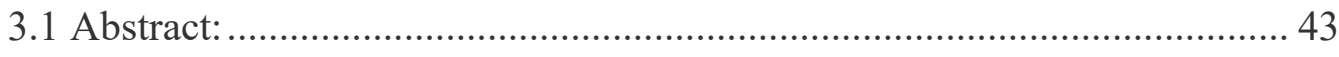

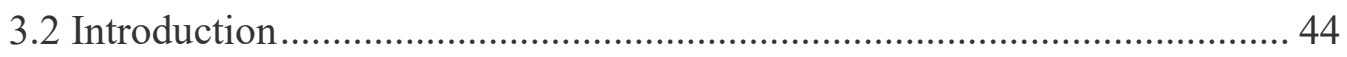

3.3 Materials and Methods.......................................................................... 47

3.3.1 Preparing socks for in-situ impregnation (Kier boiling)........................ 48

3.3.2 In-situ impregnation of Ag nanoparticles into socks (laboratory-

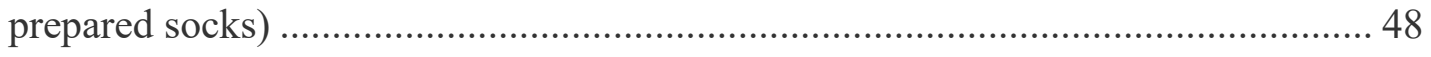

3.3.3 Day-to-day wear with sweat, bleach, and detergent ............................ 49

3.3.4 Wastewater exposure ........................................................................ 49

3.3.5 Sample preparation for ICP-MS analysis .......................................... 50

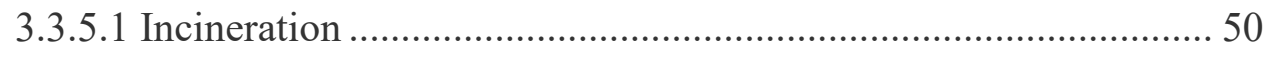

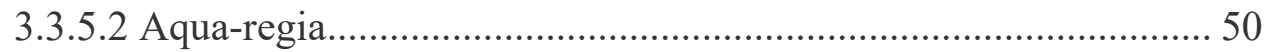

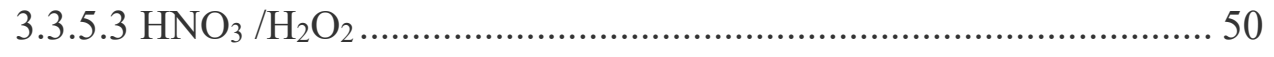

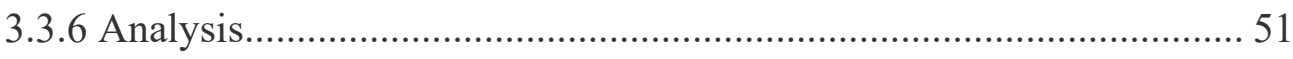

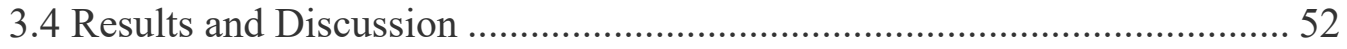

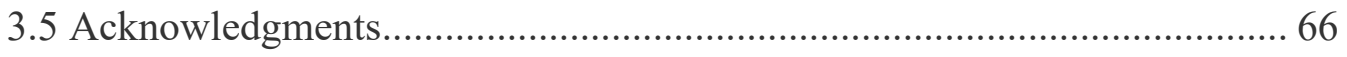

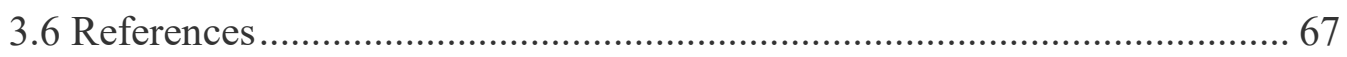

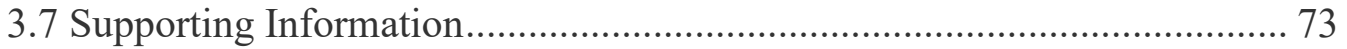

Chapter 4: Release and Transformation of Zinc Oxide Nanoparticles from Commercial Products Throughout Their Life Cycle ...................................................... 81

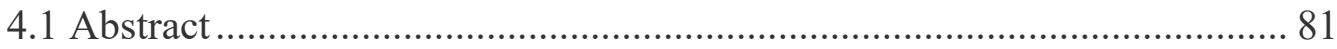

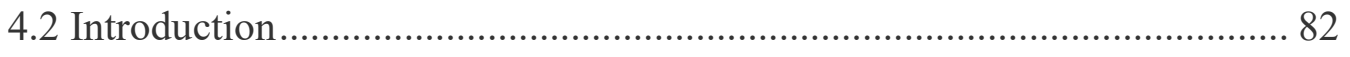

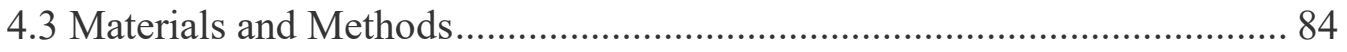

4.3.1 $\mathrm{ZnO}$ nanoparticle synthesis ................................................................. 85 


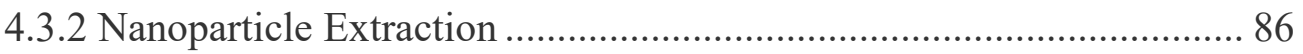

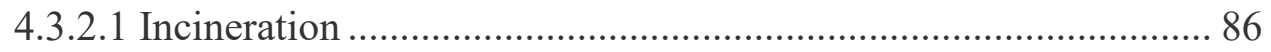

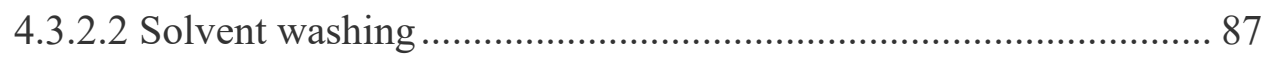

4.3.3 Day-to-day use scenarios with bleach, chlorine, detergent, sweat. ..... 87

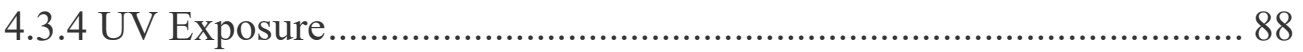

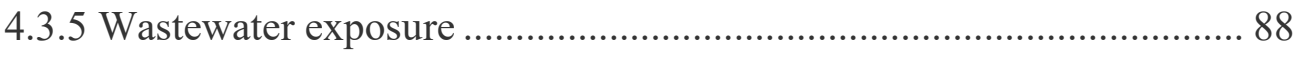

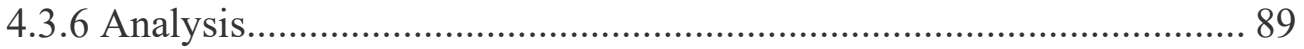

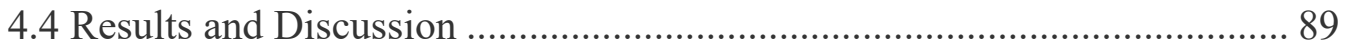

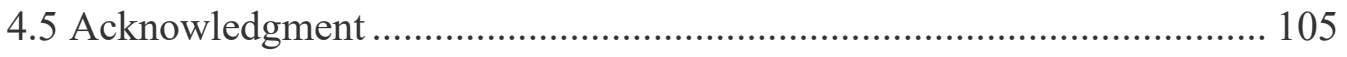

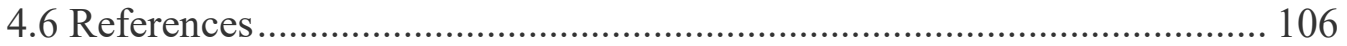

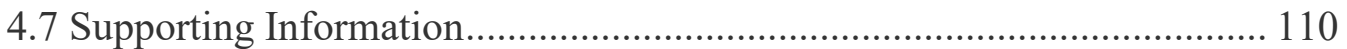

Chapter 5: Transformation of Cerium Oxide nanoparticles during potential use scenarios from product incorporation to collection in the wastewater stream................ 127

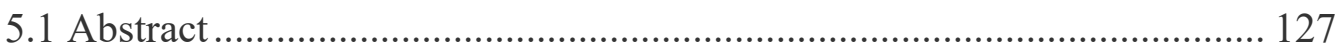

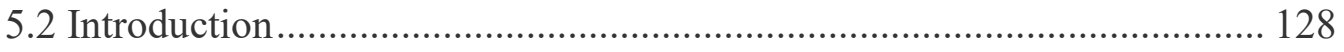

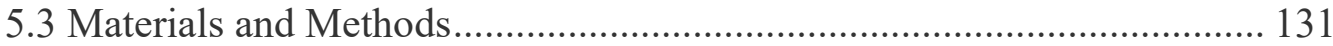

5.3.1 Cleaning of Glassware with Aqua-Regia.......................................... 132

5.3.2 Synthesis of Cerium Oxide Nanoparticles ........................................ 132

5.3.3 Washing of Cerium Oxide Nanoparticles ......................................... 133

5.3.4 Preparation of Ceria-doped Sunscreen.................................................... 134

5.3.5 Nanoparticle Extraction ................................................................... 134

5.3.5.1 Extraction Through Incineration................................................. 134

5.3.5.2 Water Extraction ..................................................................... 134

5.3.5.3 Liquid-Liquid Extraction ............................................................ 135

5.3.6 Weathering of nanoparticles ............................................................ 135

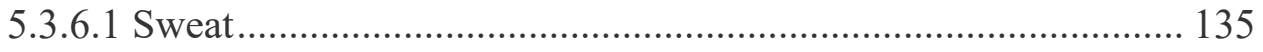

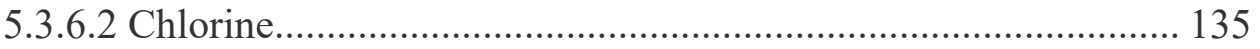

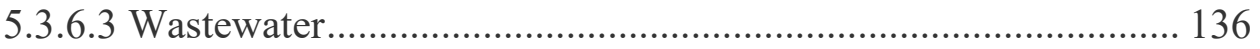

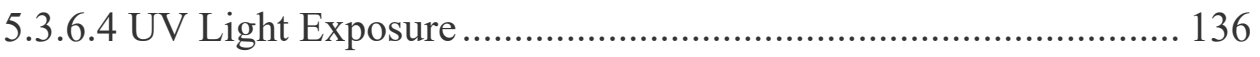

5.3.7 Preparation of Samples for TEM, EDS, and XPS Analysis .............. 136

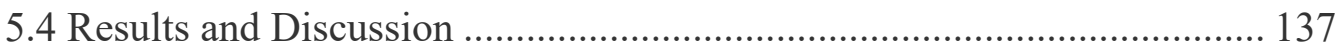


5.4.1 Synthesis of Pure Ceria ...................................................................... 137

5.4.2 Doped-Sunscreens with nanoceria ..................................................... 139

5.4.3 Weathering of $\mathrm{CeO}_{2}$ nanoparticles through day-to-day use, $\mathrm{UV}$ and wastewater exposure ....................................................................................... 142

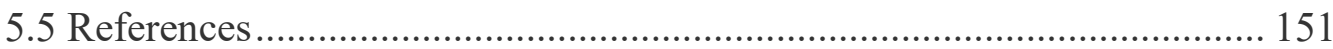

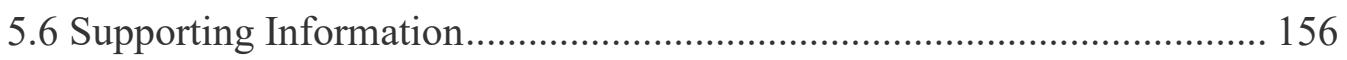

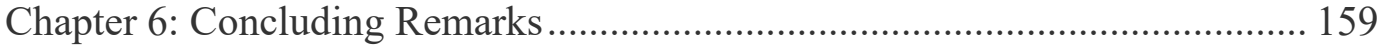

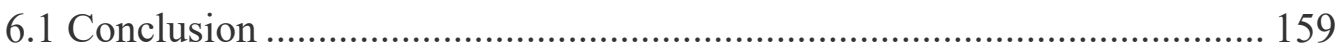

6.2 Limitations and Applications of this Research ............................................ 161

6.3 Future work recommendations ............................................................... 162

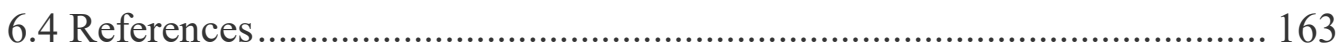




\section{List of Figures}

Figure 1-1: Pie chart showing the percentage of research articles of various classes of nanoparticles published from 2010 to 2019 from a total of 207806 articles. Data obtained from SciFinder (Last accessed December 12, 2019)

Figure 1-2: Pie chart showing the percentage of research articles of various commercial applications of nanoparticles 8284 articles. Data obtained from SciFinder (Last accessed

December 15, 2019)

Figure 1-3: The image shows the uptake of AgNPs from the soil. The toxic effects of AgNPs on the various parts of the plant cell are demonstrated ${ }^{83}$. The figure is used with permission from Tripathi under the terms of the Creative Commons Attribution License (CC BY).

Figure 1-4: TEM images of a) control, b-d) cells exposed to AgNPs. White arrows show the nanoparticles and the black arrows show the nanoparticles causing the cell membrane to rupture ${ }^{85}$. This image is used with permission from the American Chemical Society. 13

Figure 1-5: Stick and ball representation of $\mathrm{ZnO}$ crystal structures. The grey spheres are zinc atoms, and the black spheres are oxygen atoms ${ }^{92}$. This image is used with permission from AIP Publishing. 14

Figure 1-6: Shows the Standard solar spectrum (1), and a transmission spectrum of $10 \% \mathrm{CeO}_{2}$ NP sol in $20 \mu \mathrm{m}$ cuvette (4). Dashed lines correspond to the left axis; the solid line corresponds to the right axis ${ }^{107}$. This image is used with permission from Elsevier. 17

Figure 1-7: Shows the appearance of voids on the surface of $\mathrm{CeO}_{2} \mathrm{NPs}_{\mathrm{s}}$ due to oxygen vacancies ${ }^{109}$ used with permission from AIP Publishing......

Figure 2-1: Diagram of TEM components ${ }^{4}$ used with permission from North Carolina State University.

Figure 2-2: The process of generation of X-rays. (1) The electron beam knocks off one of the electrons in the atom, leaving a void. (2) The void is filled by an electron from a 
higher energy shell, causing a very specific release of energy ${ }^{16}$. This image was used with permission from ThermoFisher Scientific. 34

Figure 2-3: Schematic of the ICP-MS system ${ }^{24}$ used with permission from Springer

Vienna. 36

Figure 2-4: Photoelectron release upon irradiation with an X-ray beam in $\mathrm{XPS}^{33}$ used with permission from Elsevier. 38

Figure 3-1. Overview of the path of potential transformations for AgNPs studied in this paper. 46

Figure 3-2. TEM images of $25 \mathrm{~nm}$ pristine AgNPs from Nanocomposix in $\mathrm{ddH}_{2} \mathrm{O}$ Left: Close up view showing particles of mostly spherical shape. Right: A full view of the nanoparticle sample, indicating a reasonably monodisperse sample with little to no aggregation.

Figure 3-3. Summary of the lifecycle path and analysis of different AgNP sources studied in this paper. All analysis consists of TEM, EDS, and ICP-MS. The commercial socks were only analyzed through ICP-MS.

Figure 3-4. Mean ( \pm standard deviation) total Ag, derived from five samples, as measured through ICP-MS, based on different Ag extraction procedures on commercial socks before day-to-day use and wastewater exposure.

Figure 3-5. TEM and EDS image of a) AgNPs found in laboratory-prepared socks b) Only $\mathrm{TiO}_{2}$ and $\mathrm{ZnO}$ NPs found in commercial socks. 56

Figure 3-6. Representative TEM images of laboratory-prepared socks weathered with of a) sweat b) bleach, c) detergent for 24-hours and TEM images of pristine NPs weathered with d) sweat e) bleach and f) detergent for 24-hours to mimic day-to-day use. See supporting information Figure S 3-1 and Figure S 3-2 for EDS data

Figure 3-7. Representative TEM images of a) sweat b) bleach, c) detergent solution from laboratory-prepared socks and TEM images of d) sweat e) bleach f) detergent solution 
from pristine NPs after 24-hour incubation with wastewater. See supporting information Figure S 3-3 and Figure S 3-4 for EDS data.

Figure 3-8. XPS spectra showing the binding energy of Ag of pristine AgNPs (red) and those incubated in wastewater for 24-hours (black) obtained through high-resolution XPS.

Figure 4-1: Overview of the path of potential transformation for $\mathrm{ZnO}$ nanoparticles studied in this paper.

Figure 4-2: TEM images and EDS spectrum of ZnO NPs of sources: a) White Nanosilver Sports socks, b) White Coolibar Long Sleeve T-shirt, c) Johnson's medicated baby powder, d) Aveeno Active Naturals sensitive skin mineral sunscreen SPF 50 and e) Labsynthesized pristine nanoparticles. Samples were incinerated for the extraction of nanoparticles. 91

Figure 4-3: TEM images EDS spectrum of ZnO NPs of sources, a) Johnson's medicated baby powder b) Aveeno Active Naturals sensitive skin mineral sunscreen SPF 50. Samples were extracted through the solvent washing process. 92

Figure 4-4: TEM images and EDS spectrum of control sources: a)White Hanes Sports Cut Socks (incineration), b)White George Men's Short-Sleeve Basic T-shirt (incineration) c) Johnson's baby powder (solvent washing) d)Aveeno Baby sunscreen lotion SPF 55 (solvent washing), e)Garnier Ombrella Kids hypoallergenic lotion SPF 50+ (solvent washing) and f)Neutrogena Ultra Sheer face sunscreen SPF 60. Samples were extracted through the solvent washing process

Figure 4-5: Leaching of nanoparticles from socks, t-shirt, powder, and sunscreen when incubated in bleach, detergent, chlorine, and sweat solution for 24-hours to mimic day-today use conditions with and without UV treatment analyzed by ICP-MS. Error bars represent standard deviation with $n=9$. 101 
Figure 4-6: TEM images of a) powder, b) sunscreen, and c) pristine nanoparticles incubated in raw wastewater for 24-hours to mimic wastewater collection. Please refer to supporting information (Figure S 4-12) for EDS peaks.

Figure 5-1: Transformation of nanoparticles (left) may occur when introduced into a consumer product and then exposed to chlorination, sweat, light, and wastewater (right).

Figure 5-2: (left to right) Solution of Cerium nitrate and water; 10 seconds after the ammonium hydroxide added to the solution; Colour change to purple minutes after stirring the solution; Colour change to yellowish-white colour overnight.

Figure 5-3: DLS distribution nanoceria from initial synthesis (left) and modified synthesis technique (right). 138

Figure 5-4: TEM and EDS image of synthesized $\mathrm{CeO}_{2}$ nanoparticles. A large-scale view image can be found in Figure S 5-1 of the supporting information....

Figure 5-5: TEM images and EDS peaks of Sunscreen A (a,d), Sunscreen B (b,e), and Pristine $\mathrm{CeO}_{2}$ nanoparticles (c,f), after incubated with sewage water for 24 hours to mimic wastewater collection. Chlorine and sulfur were found in all samples via EDS analysis.

Figure 5-6: XPS spectrum (860- $935 \mathrm{eV}$ ) of cerium peaks in synthesized pristine $\mathrm{CeO}_{2}$ nanoparticles (red) compared to $\mathrm{CeO}_{2}$ nanoparticles incubated in wastewater for 24-hours (grey). The oxidation state of cerium represented by the peaks are labeled directly over the peaks ${ }^{49-51}$ 149 


\section{List of Tables}

Table 1-1: The use of the terms agglomerate and aggregate from four literature sources ${ }^{63}$.

Table 3-1. ICP-MS result of Ag content in AgNP containing socks before any use or wastewater exposure. 56

Table 3-2. Mean concentration ( \pm standard deviation), derived from triplicates, as measured through ICP-MS for Ag extracted from socks into solution after 24-hours of day-to-day use 58

Table 3-3. Summary of the type of morphological changes and chemical associations of AgNPs when pristine or embedded into the fabric and applied to various use and exposure conditions. 65

Table 4-1: TEM images of nanoparticles extracted from commercial products after subjected to day-to-day use conditions for 24-hours. Please refer to supporting information (Figure S 4-4, Figure S 4-5, Figure S 4-6, and Figure S 4-7) for EDS spectra.

Table 4-2: TEM images of nanoparticles extracted from commercial products that had been subjected to day-to-day use conditions for 24-hours after being exposed to UV light for 24-hours. Please refer to supporting information (Figure S 4-8, Figure S 4-9, Figure S 4-10, and Figure $\mathbf{S}$ 4-11) for EDS spectra. 99

Table 4-3: TEM images of nanoparticles extracted from commercial products that had been incubated with raw wastewater for 24-hours after being subjected to day-to-day use conditions. Please refer to supporting information (Figure S 4-13, Figure S 4-14, Figure S 4-15, and Figure S 4-16) for EDS spectra. Arrows indicate agglomerated particles due to dissolution. 103

Table 5-1: TEM images and EDS spectrum of $\mathrm{CeO}_{2}$ nanoparticles after impregnation into sunscreen A and sunscreen B at 24 hours, 1-week, and 2-week duration to show the stability of the nanoparticles in the sunscreen matrix. 
Table 5-2: TEM images and EDS spectrum of $\mathrm{CeO}_{2}$ nanoparticles after incubation of sunscreen A, sunscreen B, and pristine nanoparticles in sweat and chlorine for 24 hours to mimic day-to-day use. Elemental association with chlorine was observed on all samples treated with sweat through EDS.

Table 5-3: TEM images and EDS spectrum of $\mathrm{CeO}_{2}$ nanoparticles after incubation of sunscreen A, sunscreen B, and pristine nanoparticles in sweat and chlorine for 24 hours while under UV light to mimic day-to-day use. Elemental association with chlorine was observed on all samples treated with sweat + UV through EDS. Elemental association with sulfur was observed in both sunscreens treated with sweat + UV 


\section{List of Supporting Figures}

Figure S 3-1: TEM image and EDS data of extracted samples from laboratory-prepared socks exposed to a) sweat b) bleach, c) detergent. Circles indicate areas probed for EDS.

Figure S 3-2: TEM image and EDS data of pristine NPs weathered with a) sweat b) bleach and c) detergent. Circles indicate areas probed for EDS.

Figure S 3-3: TEM image and EDS data of extracted samples from a) sweat b) bleach c) detergent solutions after treatment with wastewater. Circles indicate areas probed for EDS

Figure S 3-4: TEM image and EDS data of pristine NPs treated with wastewater after weathering with a) sweat b) bleach c) detergent. Circles indicate areas probed for EDS.

Figure S 3-5: TEM image and EDS data of pristine AgNPs in a) wastewater b) sulfurrich solution c) nitrogen-rich solution d) wastewater + sulfur-rich solution e) wastewater + nitrogen-rich solution. Circles indicate areas probed for EDS. 79

Figure S 3-6: TEM image and EDS data of pristine AgNPs in wastewater with $\mathrm{pH}$ altered to a) $\mathrm{pH} 4.0 \mathrm{~b}$ ) $\mathrm{pH} 6.0 \mathrm{c}$ ) $\mathrm{pH}$ 8.0. Circles indicate areas probed for EDS.

Figure S 4-1: (top) TEM images and (bottom) EDS peaks of BEHR Ultra white paint extracted through incineration.

Figure S 4-2: SEM image and EDS spectrum of Johnson's baby powder showing ZnO around the silicon particles present in talc.

Figure S 4-3: TEM images and EDS peaks of control sources prepared by incineration: a) Johnson's baby powder, b)Aveeno Baby sunscreen lotion SPF 55, c)Garnier Ombrella Kids hypoallergenic lotion SPF 50+ and d)Neutrogena Ultra Sheer face sunscreen SPF 60 113 
Figure S 4-4: TEM images and EDS peaks of solutions of $\mathrm{ZnO}$ from t-shirt after day-today use conditions: a)sweat, b)chlorine, c)detergent and d)bleach.

Figure S 4-5: TEM images and EDS peaks of solutions of $\mathrm{ZnO}$ from baby powder after day-to-day use conditions: a) sweat, b) chlorine, c) detergent and d) bleach. 115

Figure S 4-6: TEM images and EDS peaks of solutions of $\mathrm{ZnO}$ from sunscreen after dayto-day use conditions: a) sweat, b) chlorine, c) detergent and d) bleach. 116

Figure S 4-7: TEM images and EDS peaks of solutions of $\mathrm{ZnO}$ from pristine nanoparticles after day-to-day use conditions: a)sweat, b)chlorine, c)detergent and d)bleach. 117

Figure S 4-8: TEM images and EDS peaks of solutions of $\mathrm{ZnO}$ from t-shirt after exposed to UV light and day-to-day use conditions: a)sweat, b)chlorine, c)detergent and d)bleach.

Figure S 4-9: TEM images and EDS peaks of solutions of $\mathrm{ZnO}$ from baby powder after exposed to UV light and day-to-day use conditions: a)sweat, b)chlorine, c)detergent and d)bleach. 119

Figure S 4-10: TEM images and EDS peaks of solutions of $\mathrm{ZnO}$ from sunscreen after exposed to UV light and day-to-day use conditions: a)sweat, b)chlorine, c)detergent and d)bleach. 120

Figure S 4-11: TEM images and EDS peaks of solutions of $\mathrm{ZnO}$ from pristine nanoparticles after exposed to UV light and day-to-day use conditions: a)sweat, b)chlorine, c)detergent and d)bleach.

Figure S 4-12: TEM images and EDS peaks of a) baby powder, b) sunscreen and c) pristine nanoparticles in wastewater.

Figure S 4-13: TEM images and EDS peaks of mixture of wastewater and solutions of tshirt after day-to-day conditions are applied a) sweat, b) chlorine, c) detergent and d) bleach. 
Figure S 4-14: TEM images and EDS peaks of mixture of wastewater and solutions of baby powder after day-to-day conditions are applied a) sweat, b) chlorine, c) detergent

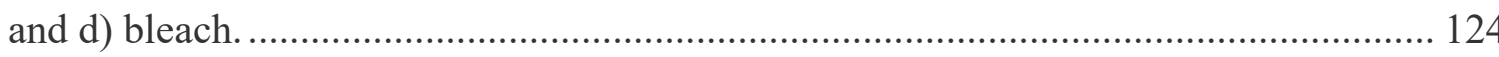

Figure S 4-15: TEM images and EDS peaks of mixture of wastewater and solutions of sunscreen after day-to-day conditions are applied a) sweat, b) chlorine, c) detergent and d) bleach. 125

Figure S 4-16: TEM images and EDS peaks of mixture of wastewater and solutions of pristine nanoparticles after day-to-day conditions are applied a) sweat, b) chlorine, c) detergent and d) bleach.

Figure S 5-1: Cerium oxide nanoparticles imaged from the TEM. The EDS reveals the pure cerium oxide. 158 


\section{List of Supporting Tables}

Table S 3-1: The test results for raw wastewater composition. Sample 1 of raw wastewater was used for the pristine NPs. Sample 2 of raw wastewater was used for the solutions from day-to-day wear of laboratory-prepared socks and pristine NPs............. 73

Table S4-1: The test results for the content raw wastewater .................................. 110

Table S5-1: The test results for the content of raw wastewater ................................. 156

Table S 5-2: TEM Images and EDS peaks of $\mathrm{CeO}_{2}$ nanoparticles extracted from sunscreen A and sunscreen B after 4-weeks of incubation by incineration, solvent extraction and water extraction. 


\section{List of Abbreviations}

\begin{tabular}{|c|c|}
\hline$\%$ & percentage \\
\hline \& & and \\
\hline \pm & plus/minus \\
\hline${ }^{\circ} \mathrm{C}$ & Celsius \\
\hline$\mu g$ & Microgram \\
\hline$\mu \mathrm{L}$ & Microliter \\
\hline$\mu \mathrm{m}$ & Micrometer \\
\hline a.k.a. & also known as \\
\hline A549 & Adenocarcinomic human alveolar basal epithelial cells \\
\hline $\mathrm{Ag}$ & Silver \\
\hline $\mathrm{Ag}(\mathrm{NH} 3)^{2+}$ & Silver(I) Diamine Cation \\
\hline $\mathrm{Ag}^{+}$ & Silver Cation \\
\hline $\mathrm{Ag}_{2} \mathrm{~S}$ & Silver Sulfide \\
\hline $\mathrm{AgCl}$ & Silver Chloride \\
\hline $\mathrm{AgNO}_{3}$ & Silver Nitrate \\
\hline Ag NP & Silver Nanoparticle \\
\hline $\mathrm{Ag}$ NPs & Silver Nanoparticles \\
\hline ANOVA & ANalysis Of VAriance \\
\hline ATP & Adenosine Triphosphate \\
\hline $\mathrm{Au}$ & Gold \\
\hline B. barbaricus & Bacillus Barbaricus \\
\hline B. subtilis & Bacillus Subtilis \\
\hline BEAS-2B & Human bronchial epithelial cells \\
\hline BS & British Standards \\
\hline $\mathrm{Ca}^{2+}$ & Calcium Cation \\
\hline CCD & Charge-Coupled Device \\
\hline $\mathrm{Ce}$ & Cerium \\
\hline $\mathrm{Ce}_{2} \mathrm{~S}_{3}$ & Cerium Sulfide \\
\hline $\mathrm{Ce}^{3+}$ & Cerium $(3+)$ ion \\
\hline $\mathrm{Ce}^{4+}$ & Cerium $(4+)$ ion \\
\hline $\mathrm{CeCl}_{3}$ & Cerium (III) Chloride \\
\hline $\mathrm{CeO}_{2}$ & Cerium Oxide \\
\hline $\mathrm{CeO}_{2} \mathrm{NP}$ & Cerium Oxide Nanoparticle \\
\hline $\mathrm{CeO}_{2} \mathrm{NPS}$ & Cerium Oxide Nanoparticles \\
\hline CF300-CU & Carbon Film 300 Cupper \\
\hline $\mathrm{Cl}$ & Chlorine \\
\hline CNT & Carbon Nanotube \\
\hline CNTs & Carbon Nanotubes \\
\hline COD & Chemical Oxygen Demand \\
\hline
\end{tabular}




\begin{tabular}{|c|c|}
\hline $\mathrm{CuO}$ & Copper Oxide \\
\hline CuO NPs & Copper Oxide Nanoparticles \\
\hline D. magna & Daphnia magna \\
\hline $\mathrm{dd}_{2} \mathrm{O}$ & Double Deionized Water \\
\hline $\mathrm{dH}_{2} \mathrm{O}$ & Deionized Water \\
\hline DLD & Delay-Line Detector \\
\hline DLS & Dynamic Light Scattering \\
\hline Dr. & Doctor \\
\hline $\mathrm{E}$ & Energy \\
\hline $\mathrm{e}^{-}$ & electron \\
\hline E. coli & Escherichia coli \\
\hline e.g. & exempli gratia means "for example" \\
\hline $\mathrm{ECHA}$ & European Chemicals Agency \\
\hline EDS & Energy Dispersive X-ray Spectroscopy \\
\hline etc. & et cetera means "and other similar things" \\
\hline eV & Electron volt \\
\hline $\mathrm{Fe}_{2} \mathrm{O}_{3}$ & Iron (III) Oxide \\
\hline FEI & Field Electron and Ion \\
\hline g & Gram \\
\hline $\mathrm{H}$ & Hydrogen \\
\hline $\mathrm{h}$ & constant factor \\
\hline $\mathrm{H}_{2} \mathrm{O}$ & water \\
\hline $\mathrm{H}_{2} \mathrm{O}_{2}$ & Hydrogen Peroxide \\
\hline $\mathrm{H}_{2} \mathrm{~S}$ & Hydrogen Sulfide \\
\hline $\mathrm{HCl}$ & Hydrogen Chloride \\
\hline $\mathrm{He}$ & Helium \\
\hline HepG2 & human liver cancer cell line \\
\hline $\mathrm{HNO}_{3}$ & Nitric Acid \\
\hline ICP & Inductively Coupled Plasma \\
\hline ICP-MS & Inductively Coupled Plasma-Mass Spectrometry \\
\hline in vitro & "within the glass" \\
\hline in vivo & "within the living" \\
\hline ISO & International Organization for Standardization \\
\hline K & Kelvin \\
\hline $\mathrm{K}^{+}$ & Potassium cation \\
\hline K. pneumoniae & Klebsiella Pneumoniae \\
\hline KE & Kinetic Energy \\
\hline kV & kilovolt \\
\hline $\mathrm{L}$ & Liter \\
\hline M & Mole \\
\hline $\mathrm{m}$ & Meter \\
\hline M. luteus & Micrococcus Luteus \\
\hline
\end{tabular}




\begin{tabular}{|c|c|}
\hline MA & Massachusetts \\
\hline $\mathrm{Mg}$ & Milligram \\
\hline $\mathrm{Mg}^{2+}$ & Magnesium Cation \\
\hline Min & Minute \\
\hline Mins & Minutes \\
\hline $\mathrm{mL} / \mathrm{ml}$ & Milliliter \\
\hline $\mathrm{mM}$ & Millimole \\
\hline Mmol & Millimole \\
\hline MRI & Magnetic Resonance Imaging \\
\hline $\mathrm{mV}$ & Millivolt \\
\hline $\mathrm{N}$ & number \\
\hline $\mathrm{N}$ & Nitrogen \\
\hline $\mathrm{Na}^{+}$ & Sodium Cation \\
\hline $\mathrm{Na}_{2} \mathrm{CO}_{3}$ & Sodium Carbonate \\
\hline $\mathrm{NaCl}$ & Sodium Chloride \\
\hline $\mathrm{NaClO}$ & Sodium Hypochlorite \\
\hline $\mathrm{NaOH}$ & Sodium Hydroxide \\
\hline $\mathrm{Ng}$ & Nanogram \\
\hline $\mathrm{NH}_{3}-\mathrm{N}$ & Ammonia \\
\hline $\mathrm{Nm}$ & Nanometer \\
\hline $\mathrm{NO}_{3}-\mathrm{N}$ & Nitrate \\
\hline NP & Nanoparticle \\
\hline NPs & Nanoparticles \\
\hline NTU & nephelometric turbidity unit \\
\hline $\mathrm{O}_{2}$ & Oxygen \\
\hline OECD & Organization for Economic Co-operation and Development \\
\hline $\mathrm{OH}^{-}$ & Hydroxide ion \\
\hline $\mathrm{P}$ & Probability \\
\hline $\mathrm{P}$ & Phosphorus \\
\hline PA & Pennsylvania \\
\hline $\mathrm{Pg}$ & Picogram \\
\hline $\mathrm{pH}$ & is a measure of the hydrogen ion concentration of a solution \\
\hline Ph.D. & Doctor of Philosophy \\
\hline $\mathrm{PO}_{4}{ }^{3-}$ & Phosphate \\
\hline Ppb & Parts Per Billion \\
\hline Psi & Pounds per Square Inch \\
\hline Q & Quality \\
\hline REACH & $\begin{array}{l}\text { Registration, Evaluation, Assessment and Restriction of } \\
\text { Chemicals }\end{array}$ \\
\hline ROS & Reactive Oxygen Species \\
\hline Rpm & Rotations Per Minute \\
\hline S & Supporting \\
\hline
\end{tabular}




\begin{tabular}{|c|c|}
\hline S & Sulfur \\
\hline SEM & Scanning Electron Microscope \\
\hline $\mathrm{SiO}_{2} \mathrm{NPs}$ & Silica Nanoparticles \\
\hline $\mathrm{SO}_{4}^{-}$ & Sulphate \\
\hline sol & Solution \\
\hline SPF & Sun Protection Factor \\
\hline SP-ICP-MS & $\begin{array}{l}\text { Single Particle Inductively Coupled Plasma-Mass } \\
\text { Spectrometry }\end{array}$ \\
\hline TEM & Transmission Electron Microscopy \\
\hline TEMCO & Tasmanian Electro Metallurgical Company \\
\hline $\mathrm{TiO}_{2}$ & Titanium Dioxide \\
\hline $\mathrm{TiO}_{2} \mathrm{NPs}$ & Titanium Dioxide Nanoparticles \\
\hline UK & United Kingdom \\
\hline USA & United States of America \\
\hline USP & United States Pharmacopoeia \\
\hline UV & Ultraviolet \\
\hline UVA & Ultraviolet A \\
\hline UVB & Ultraviolet B \\
\hline UVC & Ultraviolet C \\
\hline W & Watt \\
\hline$w / v$ & Weight by Volume \\
\hline$w / w$ & Weight Per Weight \\
\hline $\mathrm{x}$ & times \\
\hline XANES & X-ray Absorption Near Edge Spectroscopy \\
\hline XPS & X-ray Photoelectron Spectroscopy \\
\hline$X X L$ & Extra Extra Large \\
\hline $\mathrm{Zn}$ & Zink \\
\hline $\mathrm{Zn}\left(\mathrm{O}_{2} \mathrm{CCH}_{3}\right)_{2}\left(\mathrm{H}_{2} \mathrm{O}\right)_{2}$ & Zinc acetate dihydrate \\
\hline $\mathrm{Zn}^{2+}$ & Zink Cation \\
\hline $\mathrm{ZnCl}_{2}$ & Zinc Chloride \\
\hline $\mathrm{ZnO}$ & Zinc Oxide \\
\hline ZnO NP & Zinc Oxide nanoparticle \\
\hline ZnO NPs & Zinc Oxide nanoparticles \\
\hline $\mathrm{ZnS}$ & Zinc Sulfide \\
\hline v & frequency \\
\hline
\end{tabular}




\section{Chapter 1: Introduction}

\subsection{Overview}

Nanotechnology, the science and engineering conducted at the nanoscale, has been transformative and its innovations have enabled products that are touching every aspect of our lives, from energy, to health, to electronics, and beyond ${ }^{1-5}$. Inevitably, the incorporation of nanomaterials into consumer products has led to the release of nanoparticles into the terrestrial and aqueous environments ${ }^{6-8}$. It is estimated that globally, 69200, 51000, and 8100 metric tons per year of engineered nanomaterials are released into water, soil, and air respectively ${ }^{9}$. Coupled with studies that nanomaterials may have toxic impacts on our ecosystems and our own health, greater efforts are being made to understand the behavior of released nanoparticles, the potential transformations that could take place during their lifecycle, and their ultimate effects on our health and environment $^{10-12}$.

Widespread adoption of nanomaterials in commercial applications is made possible by more efficient and cost-effective synthesis methods ${ }^{13}$. Silver nanoparticles (AgNPs) are found in a variety of consumer products due to their antibacterial capabilities ${ }^{14}$. Zinc oxide nanoparticles (ZnONPs) are widely used in commercial products for their antimicrobial ${ }^{15}$ and UV absorbing capability ${ }^{16}$. Industrial-scale production of cerium oxide nanoparticles $\left(\mathrm{CeO}_{2} \mathrm{NPs}\right)$ is expected to increase due to its use as a reducing agent to catalyze reactions ${ }^{17}$, UV light absorbent in UV light blocking products $^{18}$, and an abrasive in the glass ${ }^{19}$ and semiconductor ${ }^{20}$ industry. 
One can predict that a variety of chemical and morphological transformations could take place as a nanomaterial is first prepared in pristine form, then incorporated into a nano-enabled product, then that product is used, and eventually, the product is disposed of. For example, nanoparticles might aggregate upon introduction in a product matrix $^{21}$. During use, exposure to UV light can alter the size, surface charge, surface chemistry, and dissolution rate of $\mathrm{AgNPs}^{22}$. The pristine or modified nanoparticles might also be released from their consumer product of origin into the environment, for example, during laundering of textiles ${ }^{23,24}$ or from washing off personal care and cosmetic products $^{25}$. The majority of released nanoparticles are discharged into wastewater sewer systems and transported to wastewater treatment plants. Within the wastewater collection

stream, they will be exposed to a variety of chemical agents ${ }^{26}$, which could lead to further transformations ${ }^{27}$.

\subsection{The objective of the study}

The objective of this study is to explore the preparation of a more relevant model "weathered nanoparticle" sample that can be used in place of pristine nanoparticles for environmental studies seeking to inform future regulatory decision making. This is achieved by investigating the morphological and compositional transformations of nanoparticles in commercial products as they are put through product-specific day-to-day use scenarios followed by the leaching of these nanoparticles into the wastewater collection stream. Towards this goal, the following chapters demonstrate that Ag (Chapter 3), $\mathrm{ZnO}$ (Chapter 4), and $\mathrm{CeO}_{2}$ (Chapter 5) nanoparticles exhibit both physical 
and chemical changes when exposed to a simulated product life cycle. The commercial products are exposed to sweat as a result of human contact, UV light to mimic the sun's rays, chlorinated water to mimic a swimming pool, bleach, and detergent to simulate laundering and incubation in wastewater as the leached nanoparticles will make its way to the wastewater treatment facility. Nanoparticle transformation comparisons will be made between the use of pristine nanoparticles and commercial products containing such nanoparticles. Moreover, the extent to which each weathering step affects the physical and chemical integrity of the nanoparticles will be explored.

It is expected that identifying the modified form of the nanoparticles will result in new findings and contributions regarding a more appropriate model system for studying the health and environmental impacts of nanoparticles. Moreover, an insight towards the transformed form of nanoparticle entering the sewage treatment plant will help in the development of new treatment techniques. The weathering of commercial products containing nanoparticles with day-to-day use scenarios and through the wastewater collection system will expose the nanoparticles to varying $\mathrm{pH}$, UV radiation, various organic and inorganic molecules,, as well as an oxidizing and reducing environment which will cause the nanoparticles to morphologically and compositionally transform.

\subsection{Nanomaterials}

The International Organization for Standardization (ISO) denotes an object as a nanomaterial when one of the dimensions of the material is between $1 \mathrm{~nm}$ to $100 \mathrm{~nm}^{28}$. Nano-objects can be classified as nanoplates, nanofibers, and nanoparticles, with the 
latter having all 3 dimensions less than $100 \mathrm{~nm}^{28}$. Nanoparticles are playing an important part in shaping the $21^{\text {st }}$ century, where many countries are heavily investing in the research and development of nanoparticle technology ${ }^{29}$.Error! Reference source not found. Figure 1-1 shows the percentage of research articles published from 2010 to 2019, curated from the SciFinder database, for carbon-based, metal and metal-metal oxide, ceramic, semiconductor, and polymer nanoparticles from a total of 207806 articles on nanoparticles.

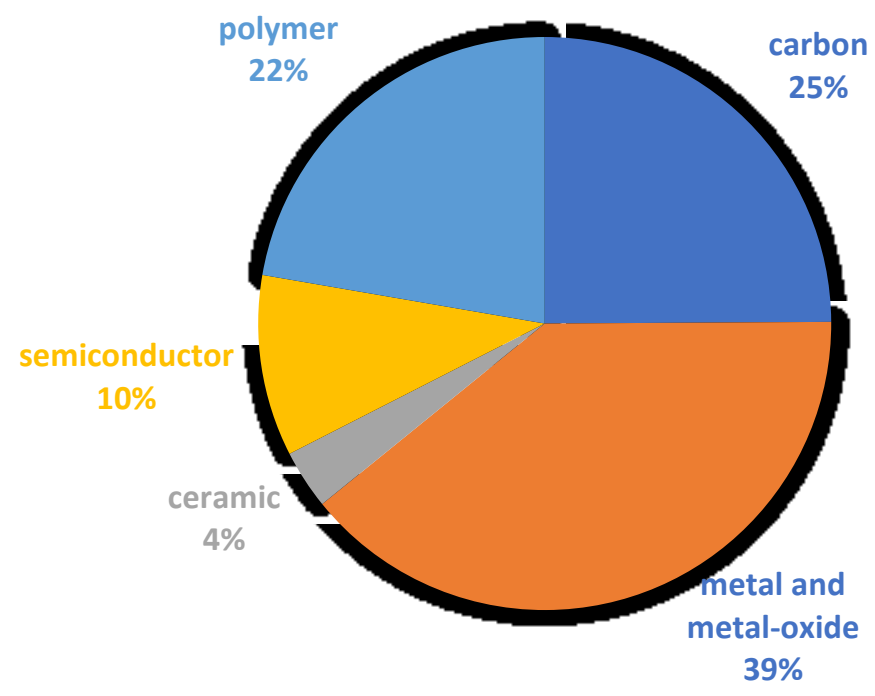

Figure 1-1: Pie chart showing the percentage of research articles of various classes of nanoparticles published from 2010 to 2019 from a total of 207806 articles. Data obtained from SciFinder (Last accessed December 12, 2019)

Given the ever-increasing discoveries in nanotechnology and their favorable properties, nanomaterials are increasingly adopted into commercial products.

Nanoparticles are integrated into commercial products for their color, antimicrobial properties, conductivity, and UV absorbance property, which can be vastly superior to 
that of the analogous bulk materials ${ }^{30-32}$. Figure 1-2 shows the percentage of research articles on the commercial applications of nanoparticles, curated from the SciFinder database, for electronics, foods and beverages, home and garden, health and fitness, the clothing industry, and the automotive industry from a total of 8284 articles.

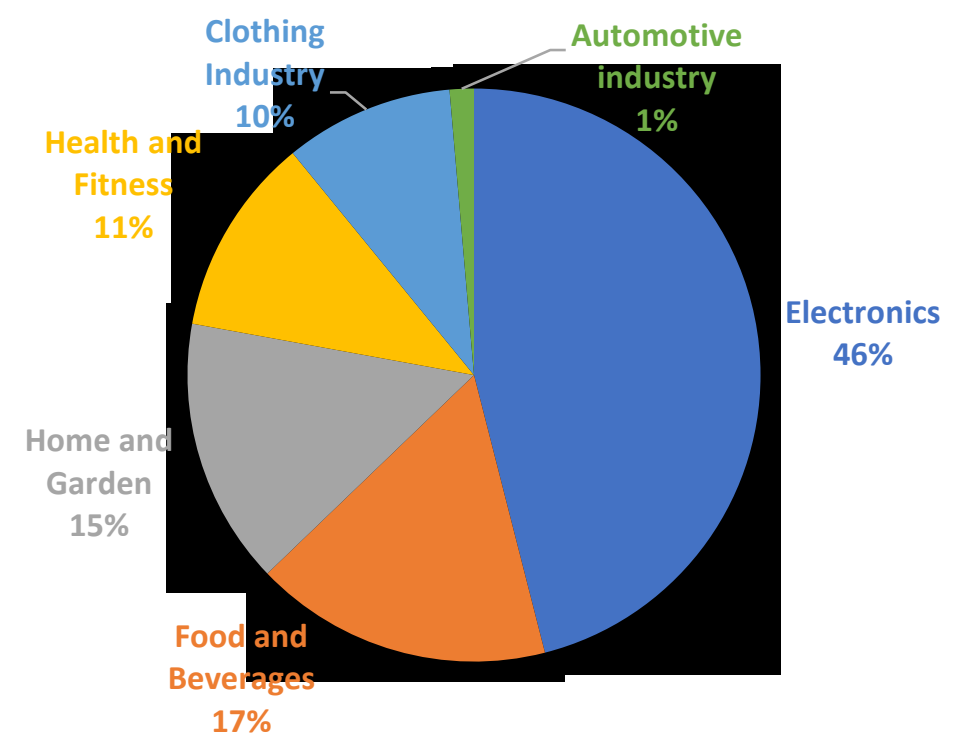

Figure 1-2: Pie chart showing the percentage of research articles of various commercial applications of nanoparticles 8284 articles. Data obtained from SciFinder (Last accessed December 15, 2019)

The small dimensions of nanoparticles instill new properties that are unique from the bulk material. These distinct properties have enabled the discovery of new inventions and further improvement to many existing products ${ }^{33}$. These properties are usually sizedependent, unlike a bulk material where the physical property stays constant regardless of size. For example, as the material gets smaller in size, the surface area to size ratio increases. Approaching the nanoscale, the surface area to volume ratio increases drastically, causing interesting and unexpected properties to arise. For example, quantum dot semiconductor nanoparticles with different core sizes $^{34}$ and different core elements ${ }^{35}$ 
would emit different distinct wavelengths of visible light when excited by UV light, due to quantum confinement within the nanoparticle. Gold as a bulk material melts at $1337 \mathrm{~K}$; however, as a $2 \mathrm{~nm}$ nanoparticle, the melting point drops to $600 \mathrm{~K}^{36}$. Iron oxide, when made in the nano-scale, would be able to exhibit superparamagnetism, which is the ability to switch its magnetization direction based on temperature ${ }^{37}$.

Nanoparticles that are integrated into commercial products may eventually leach out into the soil and waterways or be washed into the wastewater collection/treatment system and eventually be released into the environment as treated sewage sludge and water. Various studies had shown that nanoparticles leach out of commercial products with only water contact ${ }^{38-40}$. Nanoparticles contaminated water and sewage sludge might be respectively used to irrigate ${ }^{41}$ and to fertilize $e^{42}$ crops, causing nanoparticle exposure. When nanoparticles make their way to the environment, bioaccumulation into the food chain had been shown by various studies. For example, $\mathrm{CeO}_{2} \mathrm{NPs}$ had been shown to accumulate in cilantro ${ }^{43}$, soy bean ${ }^{44}$, corn $^{45}$, and tomato ${ }^{46}$ plants. The uptake of nanoparticles by plants is not just limited to $\mathrm{CeO}_{2} \mathrm{NPs}^{47} \cdot \mathrm{Ag}^{48}$ and $\mathrm{CuO}^{49} \mathrm{NP}$ accumulation had also been found in plants.

\subsubsection{Current Standing on Nanoparticle Toxicity}

Numerous standardized methods exist for the ecotoxicological regulation of Chemical Substances. Internationally harmonized standardized tests include the Organization for Economic Co-operation and Development (OECD) tests ${ }^{50}$ and International Organization for Standardization (ISO), water quality acute toxicity test ${ }^{51}$. 
Ecotoxicological test protocols are also developed by individual countries, for example, American Society of Testing and Materials Committee on Biological Effects and Environmental Fate has written protocols for testing substances on a range of aquatic invertebrates and fish ${ }^{52}$, as well as the Registration, Evaluation, Assessment and Restriction of CHemicals (REACH) regulation by the European Chemicals Agency (ECHA) for alternative substance testing to reduce test on animals ${ }^{53}$. Research on the toxicity of nanomaterials is striving to keep up with the fast pace of research on the properties and applications of nanoparticles. Standardized toxicity testing methods developed for bulk materials may not be applicable for nanoparticles, creating the need to develop new testing methods ${ }^{54,55}$. Early research on nanoparticle toxicity had demonstrated the problem with the absence of standardized toxicity testing methods for nanotoxicity studies ${ }^{56}$. Recently, the Organization for Economic Co-operation and Development (OECD) has published new guidelines for the toxicological testing of nanomaterials ${ }^{57,58}$.

There have been many mechanistic cellular injury responses documented that are thought to be caused by nanoparticles. To list a few, $\mathrm{TiO}_{2} \mathrm{NPs}$ and $\mathrm{CuO}$ NPs are known to cause oxidative stress, $\mathrm{ZnO}$ NPs and $\mathrm{CuO}$ NPs can release toxic metal ions through dissolution, polystyrene NPs can cause cationic injury to surface membranes and organelles, Carbon nanotubes (CNTs) can cause lung fibrosis, CuO NPs causes mitochondrial dysfunction that prevents Zebrafish embryo from hatching, and AgNPs and $\mathrm{SiO}_{2} \mathrm{NPs}$ can cause cell membrane lysis ${ }^{59}$. A study focused on ranking the potency of nanomaterials had shown high toxicity levels and a similar toxicity pathway between various forms of $\mathrm{CNT}$ and $\mathrm{TiO}_{2} \mathrm{NPs}$ when in vivo studies were done in the lungs of mice. 
All variant nanoparticles will need to be treated equally in toxicity testing as there is not enough data to isolate certain particles as low in toxicity ${ }^{60}$.

Nanoparticles impart their toxicity by its nanoparticle form and by its ionic form achieved through dissolution. AgNPs had been shown to be lethally toxic to Daphnia Magna in both powder and colloidal form. However, the colloidal form of AgNPs was 47 times more toxic than the powder form ${ }^{61}$. Another study on the mechanisms of toxicity of AgNPs towards HeLA cells had shown that AgNPs penetrating the cells get accumulated in the lysosomes causing some membrane damage. The acidic environment in the cell leads to the formation of $\mathrm{Ag}^{+}$ions which then induces cell death ${ }^{62}$. In another study, rats were exposed to $\mathrm{CuNPs}$ and $\mathrm{Cu}$ ions. The $\mathrm{Cu}$ ions required 3 times less in concentration to induce liver and kidney failure when compared to $\mathrm{CuNPs}^{63}$. The ionic form of nanoparticles had been shown to be more toxic than the nanoparticle form.

Priority is often given to studying how nanoparticles could have an impact on human health. It is also important that consideration is given to the fate of nanoparticles when they enter the environment ${ }^{64}$. While in recent years, a large focus has been on the aquatic environment, the impact on the terrestrial environment is increasingly under scrutiny ${ }^{55}$. Studying the long-term effects of nanoparticles, either for human health or for the environment, requires not only understanding the pristine form of the nanoparticles that are being used, but consideration must also be placed on the life cycle of the nanoparticles that can cause various transformations. The reactive and unstable nature of most nanoparticles makes transformations of these nanoparticles inevitable throughout the lifecycle of the nanoparticle ${ }^{65}$. It is important to thoroughly investigate these transformations to identify the effects of all the by-products of the nanoparticle lifecycle. 


\subsubsection{Nanoparticle Transformations}

Nanoparticles in commercial products are exposed to different stimuli that weather the nanoparticles throughout the product lifecycle. The term weather is used in this research to state the occurrence of a morphological or compositional change due to an external stimulus. With products like paint, sunscreen, sports clothing, baby powder, and cosmetics, the nanoparticles can be exposed to UV light, sweat, chlorinated water (swimming pool), laundry detergent, and laundry bleach through everyday use. Moreover, nanoparticles that leach out of the products will wash into the sewer system and be exposed to various treatments at the wastewater treatment plant. The various life cycle scenarios will cause the morphological and compositional transformation of the nanoparticles.

\subsubsection{Morphological Transformation}

Nanoparticles had been found to aggregate and agglomerate when exposed to various external factors. The definition of the terms aggregates and agglomerates had been used interchangeably, causing confusion in various applications (Table 1-1Error!

Reference source not found.). In this thesis, the term aggregate is defined as particles that are loosely bound by contact and are readily dispersible. The term agglomerate is defined as particles that are rigidly bound by fusion and are not readily dispersible. Aggregation in nanoparticles occurs when surface interactions like Van der Walls forces hold the nanoparticles together. Agglomeration can be initiated by the dissolution of nanoparticles into solution followed by precipitation into a large agglomerate. 
Table 1-1: The use of the terms agglomerate and aggregate from four literature sources ${ }^{66}$.

\begin{tabular}{|c|c|c|}
\hline Sources & $\begin{array}{c}\text { Particles that are loosely } \\
\text { bound by contact. } \\
\text { Readily dispersed. }\end{array}$ & $\begin{array}{c}\text { Particles that are rigidly } \\
\text { bound by fusion. } \\
\text { Not readily dispersed }\end{array}$ \\
\hline $\begin{array}{c}\text { Chambers Science and } \\
\text { Technology Dictionary }\end{array}$ & Aggregate & Agglomerate \\
\hline $\begin{array}{c}\text { The United States } \\
\text { Pharmacopoeia, USP 2468 }\end{array}$ & Aggregate & Agglomerate \\
\hline $\begin{array}{c}\text { British Standards } \\
\text { Institution, BS 2955 }\end{array}$ & Aggregate & Agglomerate \\
\hline $\begin{array}{c}\text { International Organization } \\
\text { for Standards, ISO 14887 }\end{array}$ & Agglomerate & Aggregate \\
\hline
\end{tabular}

\subsubsection{Compositional Transformation}

Compositional change that can occur to nanoparticles includes the reduction or oxidation of the nanoparticles. The oxidation or reduction of nanoparticles can cause ion formation that would lead to the dissolution of nanoparticles ${ }^{71,72}$. Redox reactions with metal nanoparticles can cause the formation of sulfide, chloride, amine, and thiol salts ${ }^{73,74}$. Moreover, the cations like $\mathrm{Ca}^{2+}$ and $\mathrm{Mg}^{2+}$ had been found to aggregate on the surface of nanoparticles ${ }^{75}$. Furthermore, nanoparticles are also known to form passivation layers on their surface due to sulfidation, thiol interaction, and metal halide formation ${ }^{76}$.

In order to further understand the properties of $\mathrm{Ag}, \mathrm{ZnO}$, and $\mathrm{CeO}_{2} \mathrm{NPs}$, their unique properties, toxicity mechanisms will be further discussed.

\subsection{Nanoparticles Researched}

The criteria used to choose nanoparticles to be researched are as follows; nanoparticles are being mass-produced for introduction to commercial products, the 
nanoparticle has known toxic effects, a high number of research of the nanoparticle on environmental impact, and the nanoparticle undergo known transformations when exposed to external stimuli. AgNPs, $\mathrm{ZnO}$ NPs, and $\mathrm{CeO}_{2} \mathrm{NPs}$ were chosen as the first three nanoparticles to analyze the possible transformations.

\subsubsection{Silver Nanoparticles}

\subsubsection{Nanoparticle Properties}

Ag had historically been used for its hygienic and healing purposes ${ }^{77}$. AgNPs exhibit a strong antimicrobial activity, hence, leading to its presence in various consumer products like clothing, medical devices, and even home appliances ${ }^{78,79}$. Moreover, the existence of low-cost synthesis methods using various silver salts, help streamline the adoption of AgNPs in commercial products ${ }^{79}$.

\subsubsection{Toxicity}

AgNPs had been shown to be toxic to aquatic invertebrate D. magna and to trout and human hepatocyte cell lines ${ }^{47}$. The various toxic effects of AgNPs towards plant root cells is shown in Figure 1-3Error! Reference source not found.. The uptake of AgNPs into the plant cell is caused by the particles puncturing holes through the cell wall ${ }^{80}$. Translocation of AgNPs into the cell by endocytosis occurs by the creation of vesicles on the surface of the cell ${ }^{81,82}$. Upon entering, AgNPs impart physiological, biochemical, and structural toxicity within plant cells ${ }^{83}$. AgNPs also damages the DNA and RNA by breaking the phosphodiester bonds ${ }^{84,85}$. 


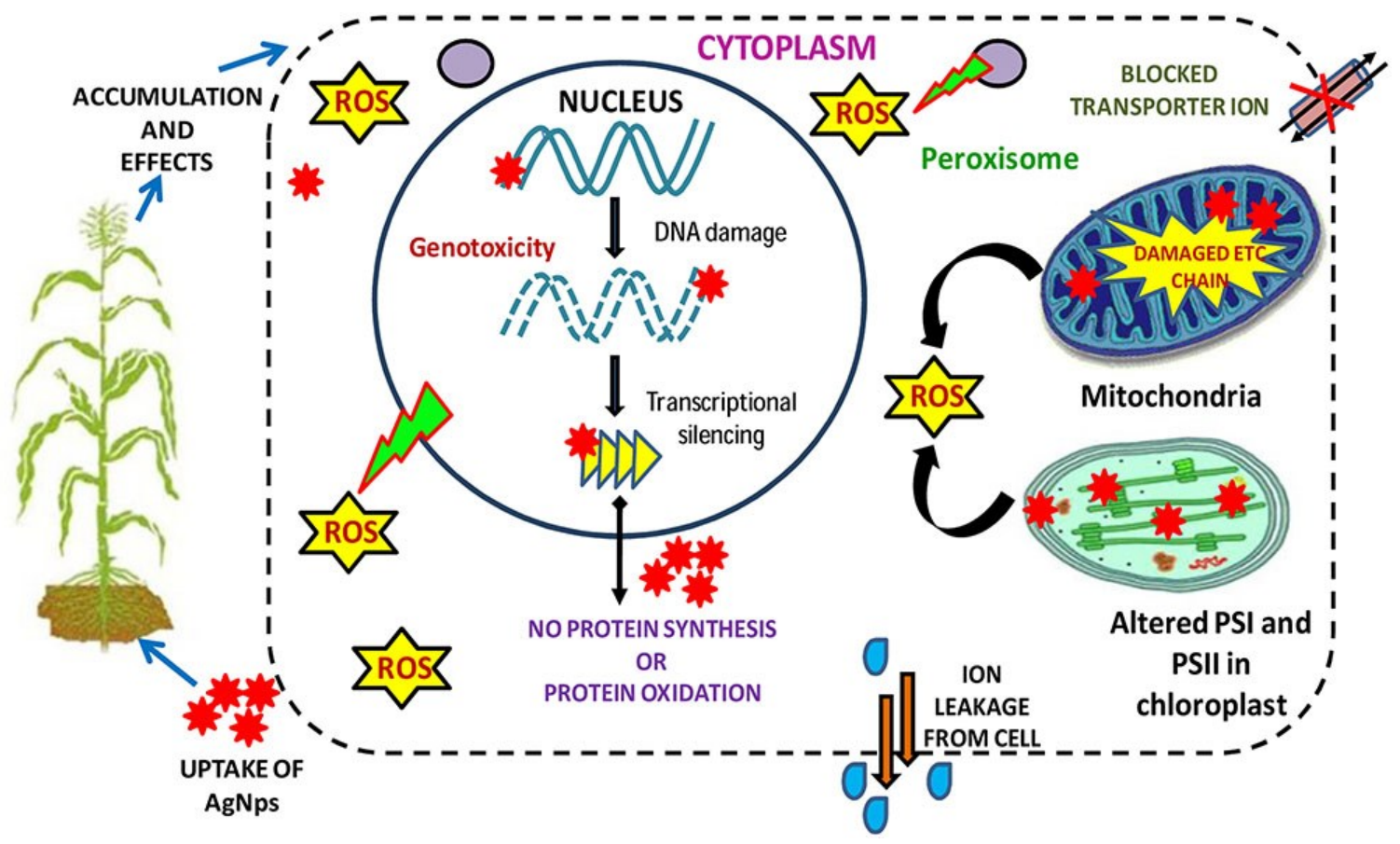

Figure 1-3: The image shows the uptake of AgNPs from the soil. The toxic effects of AgNPs on the various parts of the plant cell are demonstrated ${ }^{86}$. The figure is used with permission from Tripathi under the terms of the Creative Commons Attribution License (CC BY).

There are major ways that AgNPs exhibits its toxicity. Surface oxidation on AgNPs causes the release of toxic $\mathrm{Ag}^{+}$ions. It had been found that ionic $\mathrm{Ag}+$ inhibits basolateral $\mathrm{Na}+, \mathrm{K}+-\mathrm{ATPase}$ activity, causing acute toxicity at the gills of freshwater trout $^{87}$. AgNPs causes cellular membrane rupture when it comes in direct contact with cells. Researchers had been able to capture TEM images (Figure 1-4) of AgNPs, causing a rupture to the membrane of a gram-positive bacteria $^{88}$. Damages to the cell membrane can lead to the production of lethal ROS species with the cells ${ }^{89,90}$. 

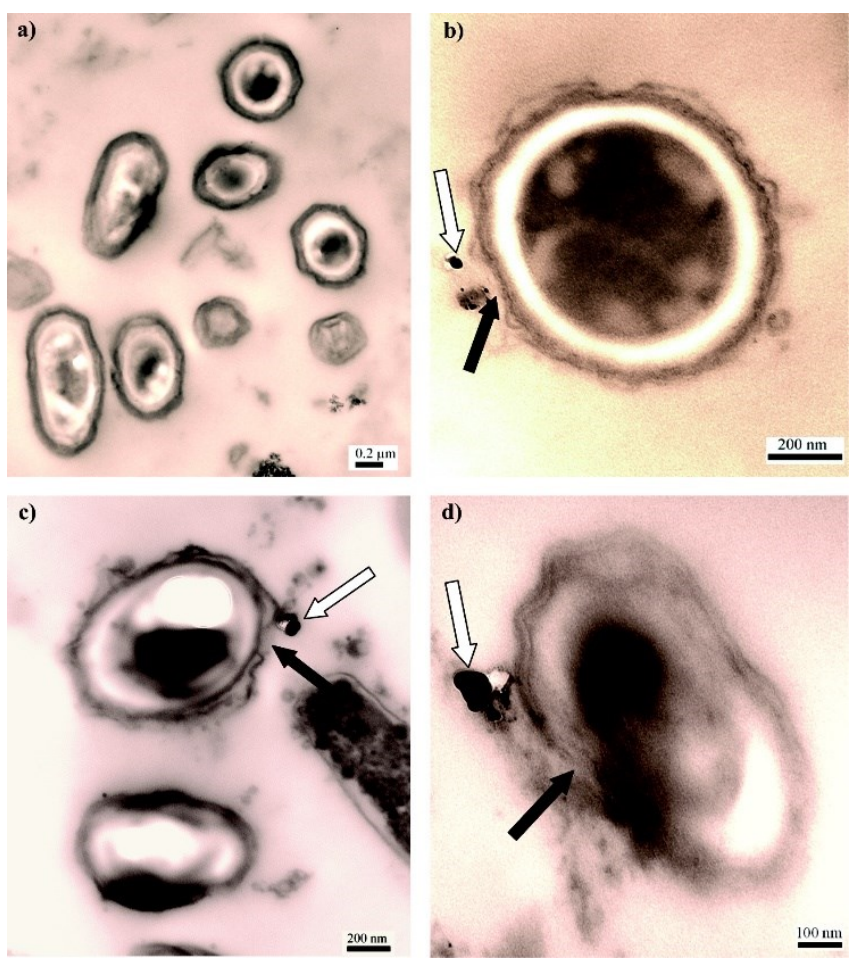

Figure 1-4: TEM images of a) control, b-d) cells exposed to AgNPs. White arrows show the nanoparticles and the black arrows show the nanoparticles causing the cell membrane to rupture ${ }^{88}$. This image is used with permission from the American Chemical Society.

\subsubsection{Known Transformations}

AgNPs oxidizes into $\mathrm{Ag}^{+}$ions in water, leading to the dissolution of $\mathrm{AgNPs}^{91}$. The ionization of AgNPs is aided by the presence of chloride ions in solution ${ }^{91}$. The transformation of $\mathrm{AgNPs}$ to $\mathrm{Ag}_{2} \mathrm{~S}$ upon a 2-hour exposure to sewage sludge had been previously reported ${ }^{92}$. Moreover, $\mathrm{AgNPs}$ had been to form $\mathrm{Ag}_{2} \mathrm{CO}_{3}, \mathrm{Ag}_{3} \mathrm{PO}_{4}$, and $\mathrm{AgCl}$ when incubated in environmental and biological solutions ${ }^{93}$. AgNPs had also been shown to form a passivation layer on the surface of the nanoparticle. The passivation layer can be composed of $\mathrm{Ag}_{2} \mathrm{~S}, \mathrm{Ag}_{2} \mathrm{O}$, silver halides, and ionic interactions with thiol groups ${ }^{76}$. 


\subsubsection{Zinc Oxide Nanoparticles}

\subsubsection{Nanoparticle Properties}

$\mathrm{ZnO}$ exists in one of three possible crystal structures; wurtzite, zinc-blende, and rock-salt, as shown in Figure 1-5Error! Reference source not found.. In the nanoparticle form, the wurtzite structure is most common ${ }^{94}$.

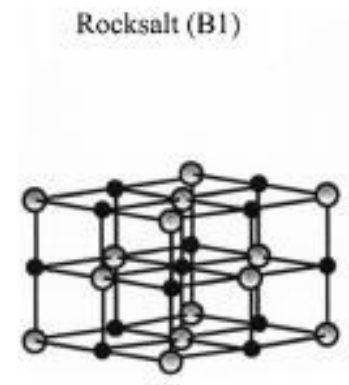

(a)

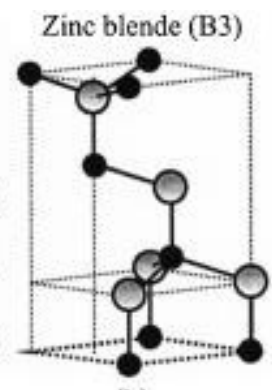

(b)

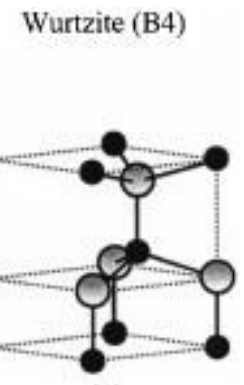

(c)

Figure 1-5: Stick and ball representation of $\mathrm{ZnO}$ crystal structures. The grey spheres are zinc atoms, and the black spheres are oxygen atoms ${ }^{95}$. This image is used with permission from AIP Publishing.

The commercial uses of ZnO NPs are due to two very important properties; being effective in absorbing UVA and UVB light ${ }^{96}$ and being an excellent antimicrobial agent $^{15}$. The UV absorbing property of $\mathrm{ZnO}$ NPs exists due to the wide, $3.37 \mathrm{eV}$ bandgap that exists at room temperature ${ }^{96}$.

\subsubsection{Toxicity}

There is a large array of literature on the adverse toxic effects of $\mathrm{ZnO}$ NPs. The cytotoxic effect of ZnO NPs had been demonstrated on numerous occasions. A complete inhibition towards the growth of $E$. coli had been shown at concentrations higher than 3.4 
$\mathrm{mM} \mathrm{ZnO} \mathrm{NPs}^{97}$. In the case of P. aeruginosa, B. subtilis, B. barbaricus, K. pneumonia, and $M$. luteus, $60 \%, 100 \%, 100 \%, 60 \%$, and $68 \%$ inhibition in bacterial growth was observed in agar plates supplemented with $100 \mu \mathrm{g}$ per plate of $\mathrm{ZnO} \mathrm{NPs}^{98}$. The $\mathrm{ZnO}$ NPs had been shown to cause acute pulmonary inflammation and peripheral immune reactions in mouse models with the severity of the immune response being dependent on the size and coating of the NPs ${ }^{99}$. Therefore, any transformation that takes place within the $\mathrm{ZnO}$ NPs will alter the degree of toxicity of the NP. The level of toxicity of ZnO NPs varies between different cell types due to the varying internal $\mathrm{pH}$ between cell types. It had been shown that $\mathrm{ZnO}$ NPs are more toxic in an acidic environment as the low $\mathrm{pH}$ promotes the dissolution of the nanoparticle ${ }^{100}$.

The dissolution chemistry of $\mathrm{ZnO}$ nanoparticles is a major contributor to its toxicity ${ }^{59}$. Researchers are working on the development of $\mathrm{ZnO}$ NP variants like zinc-iron oxides, that are more resistant to dissolution than zinc $^{59}$. The ability of ZnO NPs to generate reactive oxygen species also contributes towards the toxicity of the nanoparticles. Hydroxyl radicals are generated by ZnO NPs in aqueous suspension due to the interaction of water molecules with the surface of the nanoparticles ${ }^{101,102}$. It had been observed that $\mathrm{ZnO}$ NPs showed extreme cytotoxicity, causing apoptosis in mammalian cancer cells (HepG2, A549, and BEAS-2B) by the production of ROS while having no effects on normal cells (rat astrocytes and hepatocytes) ${ }^{103}$ suggesting the potential of $\mathrm{ZnO}$ NP as an alternative for cancer therapy. ZnO NPs can cause damage to bacterial cell membrane when it comes in direct contact. The damaged cell membrane causes an increase in membrane permeability, which leads to the accumulation of $\mathrm{ZnO}$ NPs within 
the cell ${ }^{104}$. The damage on the cell membrane is caused by electrostatic forces that attract the nanoparticle to the bacterial membrane ${ }^{105}$.

\subsubsection{Known Transformations}

$\mathrm{ZnO}$ NPs had been found to soluble in water, where the solubility increases with the decreasing particle size and a decreasing $\mathrm{pH} .7 \mathrm{~nm} \mathrm{ZnO}$ NPs at $\mathrm{pH} 7.5$ has a solubility constant of $3.2 \times 10^{-6} \mathrm{~mol} / \mathrm{L}^{106}$. Due to the highly soluble nature of ZnO NPs, under morphological conditions, $\mathrm{Zn}^{2+}$ ions bind with phosphate ions to make zinc phosphate $\left(\mathrm{Zn}_{3}\left(\mathrm{PO}_{4}\right)_{2}\right)^{107}$. Moreover, heating spherical $\mathrm{ZnO}$ NPs to $600{ }^{\circ} \mathrm{C}$ had shown to transform the nanoparticles to nanorods ${ }^{108}$.

\subsubsection{Cerium Oxide Nanoparticles}

\subsubsection{Nanoparticle Properties}

Cerium $(\mathrm{Ce})$ is the most abundant rare-earth element in the Earth's crust ${ }^{109} . \mathrm{Ce}$ can stably exist as $\mathrm{Ce}^{3+}$ and $\mathrm{Ce}^{4+}$ in solution, as well as cycle between the oxidation states depending on the environmental condition ${ }^{110}$. Figure 1-6 Error! Reference source not found. illustrates the UV shielding property of $\mathrm{CeO}_{2} \mathrm{NPs}$, where the UV irradiation wavelengths of the standard solar spectrum (Error! Reference source not found. (1)) are absorbed by the nanoparticle, resulting in the transmission spectrum (Error! Reference source not found. (4)) shown ${ }^{111}$. 


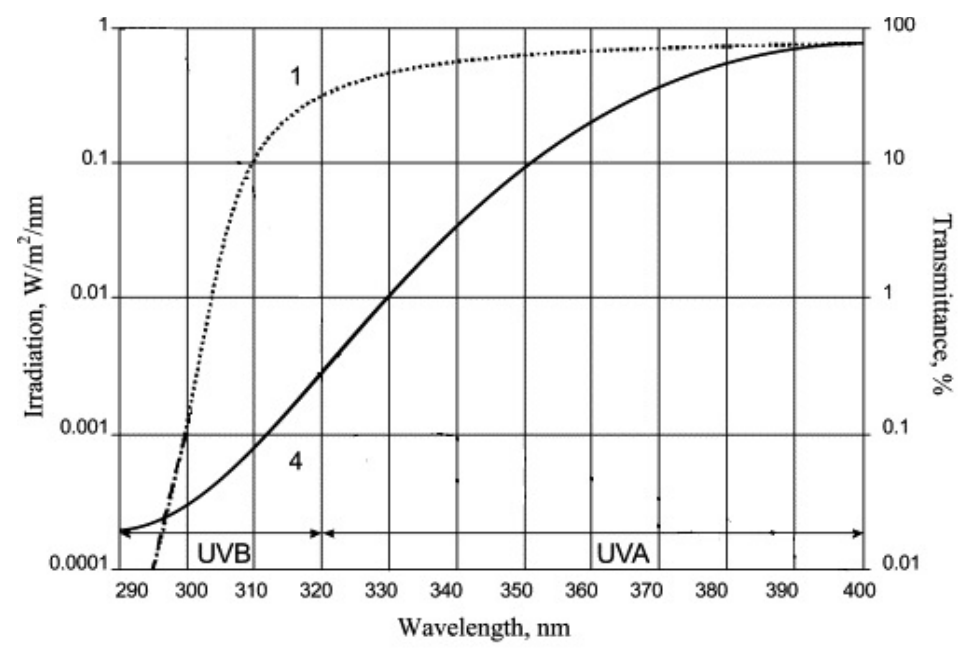

Figure 1-6: Shows the Standard solar spectrum (1), and a transmission spectrum of $10 \% \mathrm{CeO}_{2} \mathrm{NP}$ sol in $20 \mu \mathrm{m}$ cuvette (4). Dashed lines correspond to the left axis; the solid line corresponds to the right axis ${ }^{111}$. This image is used with permission from Elsevier.

$\mathrm{CeO}_{2} \mathrm{NPs}$ have the ability to exist in either a +3 (reduced) or +4 (oxidized) state at the nanoparticle surface. At the $\mathrm{Ce}^{3+}$ state, oxygen vacancies are introduced onto the surface of the nanoparticle ${ }^{112}$ (Figure 1-7Error! Reference source not found.).
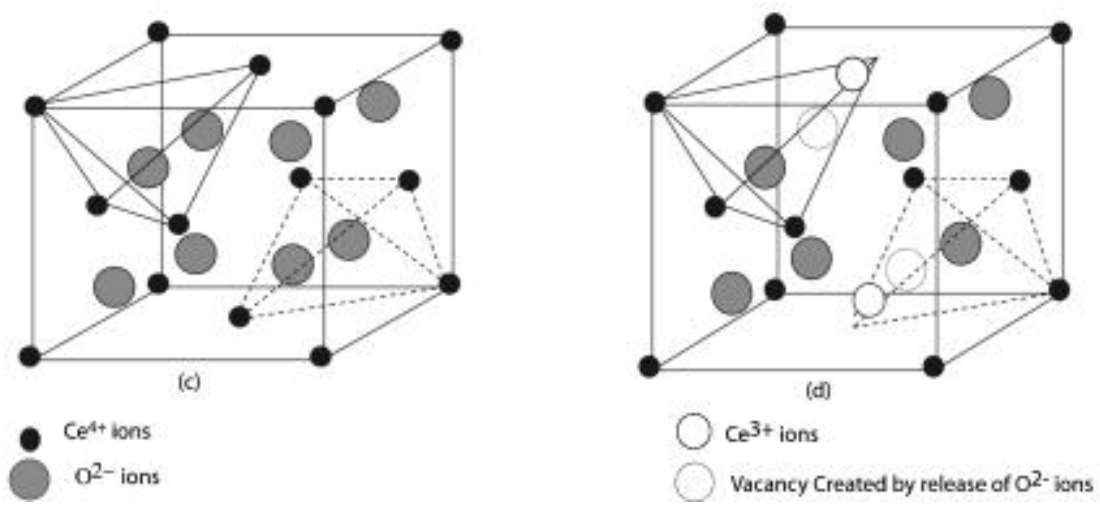

Figure 1-7: Shows the appearance of voids on the surface of $\mathrm{CeO}_{2} \mathrm{NPs}$ due to oxygen vacancies $^{113}$ used with permission from AIP Publishing. 
$\mathrm{CeO}_{2}$ are non-stoichiometric when in a nanoparticle form, giving it the ability to participate in various redox processes ${ }^{114,115} \cdot \mathrm{CeO}_{2} \mathrm{NPs}$, with its superoxide dismutase and catalase enzyme mimetic property, is able to inactivate many reactive oxygen species (ROS) like superoxide radicals ${ }^{116}$, hydrogen peroxide ${ }^{112}$, and nitroxyl radicals ${ }^{117}$.

\subsubsection{Toxicity}

There are many studies that reported the non-toxic nature of $\mathrm{CeO}_{2} \mathrm{NPs}^{43,111,118,119}$. $\mathrm{CeO}_{2}$ NPs had been shown to prevent retinal disorders leading to blindness ${ }^{120}$, increase the life span of brain cell cultures ${ }^{121}$, and inhibit cellular aging ${ }^{122}$. However, $\mathrm{CeO}_{2} \mathrm{NPs}$ do exhibit a toxic nature in some cases of extreme exposure. When tested with a Daphnia magna assay and a bioluminescent test, $\mathrm{CeO}_{2} \mathrm{NPs}$ were shown to be very toxic ${ }^{123} \cdot \mathrm{CeO}_{2}$ NPs were also shown to exhibit moderate toxicity towards human muscle tissues ${ }^{124}$, cause liver damage in rats ${ }^{125}$, and decrease the lifespan in roundworms ${ }^{126}$.

Due to the limited research resulting in the toxicity of $\mathrm{CeO}_{2} \mathrm{NPs}$, there are few findings on the mechanism of nanoparticle toxicity. Direct contact with $\mathrm{CeO}_{2} \mathrm{NPs}$ had been shown to damage the cytoplasmic membrane of algae cells ${ }^{127}$. In some cases, $\mathrm{CeO}_{2}$ NPS can also act as an oxidant that induces the formation of ROS within the cell, causing $\operatorname{apoptosis}^{128}$.

\subsubsection{Known Transformations}

$\mathrm{CeO}_{2}$ NPs had been found to dissolve into solution with a solubility constant of 23 $\mu \mathrm{mol} / \mathrm{L}$ for $9 \mathrm{~nm}$ nanoparticles when $\mathrm{pH}$ is below $4.6^{129}$. Elemental association with

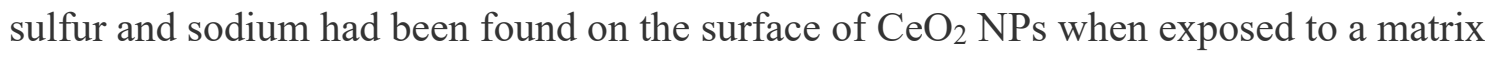


containing sulfate groups and sodium salts ${ }^{129}$. A recent study showed the transformations of $\mathrm{CeO}_{2} \mathrm{NPs}$ that took place during the combustion of a diesel engine as $\mathrm{CeO}_{2}$ nanoparticles are used as diesel fuel additives. 3-7 $\mathrm{nm} \mathrm{CeO} 2 \mathrm{NPs}$ had agglomerated to about $300 \mathrm{~nm}$ in size and a coating of various impurities had deposited on the surface of the agglomerated nanoparticle after the combustion of the diesel fuel ${ }^{130}$. 


\subsection{References}

1. Govindaraju, K., Dilip Itroutwar, P., Veeramani, V., Ashok Kumar, T. \& Tamilselvan, S. Application of Nanotechnology in Diagnosis and Disease Management of White Spot Syndrome Virus (WSSV) in Aquaculture. Journal of Cluster Science 1-9 (2019).

2. Zahedi, S. M., Karimi, M. \& Teixeira da Silva, J. A. The use of nanotechnology to increase quality and yield of fruit crops. J. Sci. Food Agric. 100, 25-31 (2020).

3. Tang, K. S. The current and future perspectives of zinc oxide nanoparticles in the treatment of diabetes mellitus. Life Sci. 239, 117011 (2019).

4. Zulfiqar, F., Navarro, M., Ashraf, M., Akram, N. A. \& Munné-Bosch, S. Nanofertilizer use for sustainable agriculture: Advantages and limitations. Plant Sci. 289, 110270 (2019).

5. Olafusi, O. S., Sadiku, E. R., Snyman, J., Ndambuki, J. M. \& Kupolati, W. K. Application of nanotechnology in concrete and supplementary cementitious materials: a review for sustainable construction. SN Appl. Sci. 1, 580 (2019).

6. Shi, W. et al. Ocean acidification increases the accumulation of titanium dioxide nanoparticles (nTiO2) in edible bivalve mollusks and poses a potential threat to seafood safety. Sci. Rep. 9, 3516 (2019).

7. Madeła, M. Impact of silver nanoparticles on wastewater treatment in the SBR. E3S Web Conf. 86, 00027 (2019).

8. Clar, J. G. et al. Release and transformation of $\mathrm{ZnO}$ nanoparticles used in outdoor surface coatings for UV protection. Sci. Total Environ. 670, 78-86 (2019).

9. Keller, A. A. \& Lazareva, A. Predicted Releases of Engineered Nanomaterials: From Global to Regional to Local. Environ. Sci. Technol. Lett. 1, 65-70 (2014).

10. Dev, A., Srivastava, A. K. \& Karmakar, S. Nanomaterial toxicity for plants. Environ. Chem. Lett. 16, 85-100 (2018).

11. Ganguly, P., Breen, A. \& Pillai, S. C. Toxicity of Nanomaterials: Exposure, Pathways, Assessment, and Recent Advances. ACS Biomater. Sci. Eng. 4, $2237-$ 2275 (2018).

12. Briffa, S. M., Lynch, I., Hapiuk, D. \& Valsami-Jones, E. Physical and chemical transformations of zirconium doped ceria nanoparticles in the presence of phosphate: Increasing realism in environmental fate and behaviour experiments. Environ. Pollut. 252, 974-981 (2019).

13. Rauwel, P., Küünal, S., Ferdov, S. \& Rauwel, E. A Review on the Green Synthesis of Silver Nanoparticles and Their Morphologies Studied via TEM. Adv. Mater. Sci. Eng. 2015, 1-9 (2015).

14. Prabhu, S. \& Poulose, E. K. Silver nanoparticles: mechanism of antimicrobial 
action, synthesis, medical applications, and toxicity effects. International Nano Letters 2, 32 (2012).

15. Pasquet, J. et al. The contribution of zinc ions to the antimicrobial activity of zinc oxide. Colloids Surfaces A Physicochem. Eng. Asp. 457, 263-274 (2014).

16. Smijs, T. G. \& Pavel, S. Titanium dioxide and zinc oxide nanoparticles in sunscreens: focus on their safety and effectiveness. Nanotechnol. Sci. Appl. 4, 95$112(2011)$.

17. Zhang, J. et al. Impacts of a Nanosized Ceria Additive on Diesel Engine Emissions of Particulate and Gaseous Pollutants. Environ. Sci. Technol. 47, 13077-13085 (2013).

18. Yabe, S. \& Sato, T. Cerium oxide for sunscreen cosmetics. J. Solid State Chem. 171, 7-11 (2003).

19. Sabia, R. \& Stevens, H. J. Performance Characterization of Cerium Oxide Abrasives for Chemical-Mechanical Polishing of Glass. Mach. Sci. Technol. 4, 235-251 (2000).

20. Feng, X. et al. Converting Ceria Polyhedral Nanoparticles into Single-Crystal Nanospheres. Science (80-. ). 312, 1504-1508 (2006).

21. Lu, P.-J., Huang, S.-C., Chen, Y.-P., Chiueh, L.-C. \& Shih, D. Y.-C. Analysis of titanium dioxide and zinc oxide nanoparticles in cosmetics. J. Food Drug Anal. 23, 587-594 (2015).

22. Mittelman, A. M., Fortner, J. D. \& Pennell, K. D. Effects of ultraviolet light on silver nanoparticle mobility and dissolution. Environ. Sci. Nano 2, 683-691 (2015).

23. Geranio, L., Heuberger, M. \& Nowack, B. The behavior of silver nanotextiles during washing. Environ. Sci. Technol. 43, 8113-8118 (2009).

24. Benn, T. M. \& Westerhoff, P. Nanoparticle silver released into water from commercially available sock fabrics. Environ. Sci. Technol. 42, 4133-9 (2008).

25. Zhang, Y., Leu, Y.-R., Aitken, R. \& Riediker, M. Inventory of Engineered Nanoparticle-Containing Consumer Products Available in the Singapore Retail Market and Likelihood of Release into the Aquatic Environment. Int. J. Environ. Res. Public Health 12, 8717-8743 (2015).

26. Gupta, V. K., Ali, I., Saleh, T. A., Nayak, A. \& Agarwal, S. Chemical treatment technologies for waste-water recycling - an overview. RSC Adv. 2, 6380 (2012).

27. Gogos, A., Wielinski, J., Voegelin, A., Emerich, H. \& Kaegi, R. Transformation of cerium dioxide nanoparticles during sewage sludge incineration. Environ. Sci. Nano 6, 1765-1776 (2019).

28. International Organization of Standards. ISO/TS 80004-2:2015(en), Nanotechnologies — Vocabulary - Part 2: Nano-objects. (2015). 
29. Hosokawa, M. Nanoparticle technology handbook. (Elsevier, 2012).

30. Khan, I., Saeed, K. \& Khan, I. Nanoparticles: Properties, applications and toxicities. Arab. J. Chem. 12, 908-931 (2019).

31. Klaus, T., Joerger, R., Olsson, E. \& Ran Granqvist, C.-G. Silver-based crystalline nanoparticles, microbially fabricated. PNAS 96, 13611-13614 (1999).

32. Li, R. et al. UV-shielding properties of zinc oxide-doped ceria fine powders derived via soft solution chemical routes. Mater. Chem. Phys. 75, 39-44 (2002).

33. Santos, C. S. C. et al. Industrial Applications of Nanoparticles - A Prospective Overview. Mater. Today Proc. 2, 456-465 (2015).

34. Ryman-Rasmussen, J. P., Riviere, J. E. \& Monteiro-Riviere, N. A. Surface Coatings Determine Cytotoxicity and Irritation Potential of Quantum Dot Nanoparticles in Epidermal Keratinocytes. J. Invest. Dermatol. 127, 143-153 (2007).

35. Zhang, F. et al. Brightly Luminescent and Color-Tunable Colloidal $\mathrm{CH}_{3} \mathrm{NH}_{3}$ $\mathrm{PbX}_{3}$ (X = Br, I, Cl) Quantum Dots: Potential Alternatives for Display Technology. ACS Nano 9, 4533-4542 (2015).

36. Schlexer, P. et al. Size-Dependence of the Melting Temperature of Individual Au Nanoparticles. Part. Part. Syst. Charact. 36, 1800480 (2019).

37. Singh, N., Jenkins, G. J. S., Asadi, R. \& Doak, S. H. Potential toxicity of superparamagnetic iron oxide nanoparticles (SPION). Nano Rev. 1, 5358 (2010).

38. Pasricha, A. et al. Comparative study of leaching of silver nanoparticles from fabric and effective effluent treatment. J. Environ. Sci. 24, 852-859 (2012).

39. Kaegi, R. et al. Release of silver nanoparticles from outdoor facades. Environ. Pollut. 158, 2900-2905 (2010).

40. Pourzahedi, L., Vance, M. \& Eckelman, M. J. Life Cycle Assessment and Release Studies for 15 Nanosilver-Enabled Consumer Products: Investigating Hotspots and Patterns of Contribution. Environ. Sci. Technol. 51, 7148-7158 (2017).

41. Norton-Brandão, D., Scherrenberg, S. M. \& van Lier, J. B. Reclamation of used urban waters for irrigation purposes - A review of treatment technologies. $J$. Environ. Manage. 122, 85-98 (2013).

42. Smith, S. R., Hall, J. E. \& Hadley, P. Composting Sewage Sludge Wastes in Relation to Their Solubility For Use As Fertilizer Materials For Vegetable Crop Production. Acta Hortic. 203-216 (1992). doi:10.17660/ActaHortic.1992.302.19

43. Morales, M. I. et al. Toxicity Assessment of Cerium Oxide Nanoparticles in Cilantro ( Coriandrum sativum L.) Plants Grown in Organic Soil. J. Agric. Food Chem. 61, 6224-6230 (2013).

44. López-Moreno, M. L. et al. Evidence of the Differential Biotransformation and

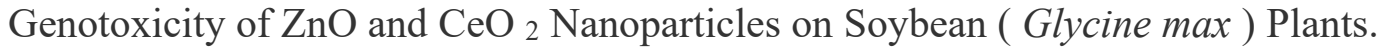


Environ. Sci. Technol. 44, 7315-7320 (2010).

45. Zhao, L. et al. Effect of surface coating and organic matter on the uptake of $\mathrm{CeO} 2$ NPs by corn plants grown in soil: Insight into the uptake mechanism. J. Hazard. Mater. 225-226, 131-138 (2012).

46. Wang, Q., Ma, X., Zhang, W., Pei, H. \& Chen, Y. The impact of cerium oxide nanoparticles on tomato (Solanum lycopersicum L.) and its implications for food safety. Metallomics 4, 1105 (2012).

47. Gaiser, B. K. et al. Assessing exposure, uptake and toxicity of silver and cerium dioxide nanoparticles from contaminated environments. Environ. Heal. 8, S2 (2009).

48. Stegemeier, J. P., Colman, B. P., Schwab, F., Wiesner, M. R. \& Lowry, G. V. Uptake and Distribution of Silver in the Aquatic Plant Landoltia punctata (Duckweed) Exposed to Silver and Silver Sulfide Nanoparticles. doi:10.1021/acs.est.6b06491

49. Peng, C. et al. Fate and Transformation of $\mathrm{CuO}$ Nanoparticles in the Soil-Rice System during the Life Cycle of Rice Plants. Environ. Sci. Technol. 51, 4907-4917 (2017).

50. Crane, M., Handy, R. D., Garrod, J. \& Owen, R. Ecotoxicity test methods and environmental hazard assessment for engineered nanoparticles. Ecotoxicology 17, 421-437 (2008).

51. International Organization for Standardization. ISO - ISO 6341:2012 - Water quality - Determination of the inhibition of the mobility of Daphnia magna Straus (Cladocera, Crustacea) - Acute toxicity test. (2018).

52. American Society for Testing and Materials. Standard Guide for Conducting Acute Toxicity Tests on Test Materials with Fishes, Macroinvertebrates, and Amphibians. (2014).

53. European Chemical Agency. Understanding REACH - ECHA. European Chemical Agency (2007). Available at: https://echa.europa.eu/regulations/reach/understanding-reach. (Accessed: 12th December 2019)

54. Labib, S. et al. Nano-risk Science: application of toxicogenomics in an adverse outcome pathway framework for risk assessment of multi-walled carbon nanotubes. Part. Fibre Toxicol. 13, 15 (2015).

55. Handy, R. D. et al. Ecotoxicity test methods for engineered nanomaterials: Practical experiences and recommendations from the bench. Environ. Toxicol. Chem. 31, 15-31 (2012).

56. Zhang, Y. et al. Critical experimental parameters related to the cytotoxicity of zinc oxide nanoparticles. J. Nanopart. Res. 16, 2440 (2014).

57. Organisation for Economic Co-operation and Development. Draft Guidance 
Document on Aquatic and Sediment Toxicological Testing of Nanomaterials. (2018).

58. Stoye, E. New guidelines to standardise toxicity testing for nanomaterials. Chemistry World (2017).

59. Nel, A. et al. Nanomaterial Toxicity Testing in the 21st Century: Use of a Predictive Toxicological Approach and High-Throughput Screening. Acc. Chem. Res. 46, 607-621 (2013).

60. Halappanavar, S. et al. Ranking of nanomaterial potency to induce pathway perturbations associated with lung responses. NanoImpact 14, 100158 (2019).

61. Asghari, S. et al. Toxicity of various silver nanoparticles compared to silver ions in Daphnia magna. J. Nanobiotechnology 10, 14 (2012).

62. De Matteis, V. et al. Negligible particle-specific toxicity mechanism of silver nanoparticles: The role of Ag+ ion release in the cytosol. Nanomedicine Nanotechnology, Biol. Med. 11, 731-739 (2015).

63. Lee, I.-C. et al. Comparative toxicity and biodistribution of copper nanoparticles and cupric ions in rats. Int. J. Nanomedicine 11, 2883-900 (2016).

64. Brar, S. K., Verma, M., Tyagi, R. D. \& Surampalli, R. Y. Engineered nanoparticles in wastewater and wastewater sludge - Evidence and impacts. Waste Manag. 30, 504-520 (2010).

65. Gottschalk, F., Sonderer, T., Scholz, R. W. \& Nowack, B. Modeled Environmental Concentrations of Engineered Nanomaterials ( $\mathrm{TiO}_{2}, \mathrm{ZnO}, \mathrm{Ag}, \mathrm{CNT}$, Fullerenes) for Different Regions. Environ. Sci. Technol. 43, 9216-9222 (2009).

66. Nichols, G. et al. A review of the terms agglomerate and aggregate with a recommendation for nomenclature used in powder and particle characterization. $J$. Pharm. Sci. 91, 2103-2109 (2002).

67. Walker, P. Chambers Dictionary of Science and Technology. (Chambers Harrap Publishers, 1999).

68. United States Pharmacopeia. USP \# 24, NF19. (United States Pharmacopeia Convention, 2000).

69. British Standards, I. BS 2955:1993, Glossary of terms relating to particle technology. (BSI Group, 1993).

70. International Organization of Standards. ISO 14887:2000(en), Sample preparation - Dispersing procedures for powders in liquids. (2000). Available at: https://www.iso.org/obp/ui/\#iso:std:iso:14887:ed-1:v1:en. (Accessed: 12th December 2019)

71. Schwabe, F. et al. Dissolution and transformation of cerium oxide nanoparticles in plant growth media. J. Nanoparticle Res. 16, 2668 (2014).

72. Bian, S.-W., Mudunkotuwa, I. A., Rupasinghe, T. \& Grassian, V. H. Aggregation 
and Dissolution of $4 \mathrm{~nm} \mathrm{ZnO}$ Nanoparticles in Aqueous Environments: Influence of pH, Ionic Strength, Size, and Adsorption of Humic Acid. Langmuir 27, 60596068 (2011).

73. Dale, A. L., Lowry, G. V \& Casman, E. A. Modeling nanosilver transformations in freshwater sediments. Environ. Sci. Technol. 47, 12920-8 (2013).

74. Fernando, I. \& Zhou, Y. Concentration dependent effect of humic acid on the transformations of silver nanoparticles. J. Mol. Liq. 284, 291-299 (2019).

75. Zhang, H., Smith, J. A. \& Oyanedel-Craver, V. The effect of natural water conditions on the anti-bacterial performance and stability of silver nanoparticles capped with different polymers. Water Res. 46, 691-699 (2012).

76. Le Ouay, B. \& Stellacci, F. Antibacterial activity of silver nanoparticles: A surface science insight. Nano Today 10, 339-354 (2015).

77. Chen, X. \& Schluesener, H. J. Nanosilver: A nanoproduct in medical application. Toxicol. Lett. 176, 1-12 (2008).

78. McShan, D., Ray, P. C. \& Yu, H. Molecular toxicity mechanism of nanosilver. $J$. Food Drug Anal. 22, 116-127 (2014).

79. Tolaymat, T. M. et al. An evidence-based environmental perspective of manufactured silver nanoparticle in syntheses and applications: A systematic review and critical appraisal of peer-reviewed scientific papers. Sci. Total Environ. 408, 999-1006 (2010).

80. Navarro, E. et al. Environmental behavior and ecotoxicity of engineered nanoparticles to algae, plants, and fungi. Ecotoxicology 17, 372-386 (2008).

81. Ovečka, M. et al. Endocytosis and vesicle trafficking during tip growth of root hairs. Protoplasma 226, 39-54 (2005).

82. AshaRani, P., Hande, M. P. \& Valiyaveettil, S. Anti-proliferative activity of silver nanoparticles. BMC Cell Biol. 10, 65 (2009).

83. Tripathi, D. K. et al. Nitric oxide alleviates silver nanoparticles (AgNps)-induced phytotoxicity in Pisum sativum seedlings. Plant Physiol. Biochem. 110, 167-177 (2017).

84. Ansari, M. A. et al. Interaction of silver nanoparticles with Escherichia coli and their cell envelope biomolecules. J. Basic Microbiol. 54, 905-915 (2014).

85. Hasani, A. et al. Metal nanoparticles and consequences on multi-drug resistant bacteria: reviving their role. SN Appl. Sci. 1, 360 (2019).

86. Tripathi, D. K. et al. Uptake, Accumulation and Toxicity of Silver Nanoparticle in Autotrophic Plants, and Heterotrophic Microbes: A Concentric Review. Front. Microbiol. 08, 7 (2017).

87. Wood, C. M., Playle, R. C. \& Hogstrand, C. Physiology and modeling of mechanisms of silver uptake and toxicity in fish. Environ. Toxicol. Chem. 18, 71- 
83 (1999).

88. El Badawy, A. M. et al. Surface Charge-Dependent Toxicity of Silver Nanoparticles. Environ. Sci. Technol. 45, 283-287 (2011).

89. Li, L., Wang, Y., Liu, Q. \& Jiang, G. Rethinking Stability of Silver Sulfide Nanoparticles (Ag2S-NPs) in the Aquatic Environment: Photoinduced Transformation of Ag2S-NPs in the Presence of Fe(III). Environ. Sci. Technol. 50, 188-196 (2016).

90. Fabrega, J., Fawcett, S. R., Renshaw, J. C. \& Lead, J. R. Silver nanoparticle impact on bacterial growth: effect of $\mathrm{pH}$, concentration, and organic matter. Environ. Sci. Technol. 43, 7285-90 (2009).

91. Kent, R. D. \& Vikesland, P. J. Controlled Evaluation of Silver Nanoparticle Dissolution Using Atomic Force Microscopy. Environ. Sci. Technol. 46, 69776984 (2012).

92. Kaegi, R. et al. Behavior of metallic silver nanoparticles in a pilot wastewater treatment plant. Environ. Sci. Technol. 45, 3902-3908 (2011).

93. Zook, J. M., Long, S. E., Cleveland, D., Geronimo, C. L. A. \& MacCuspie, R. I. Measuring silver nanoparticle dissolution in complex biological and environmental matrices using UV-visible absorbance. Anal. Bioanal. Chem. 401, 1993-2002 (2011).

94. Rekha, K., Nirmala, M., Nair, M. G. \& Anukaliani, A. Structural, optical, photocatalytic and antibacterial activity of zinc oxide and manganese doped zinc oxide nanoparticles. Phys. B Condens. Matter 405, 3180-3185 (2010).

95. Özgür, Ü. et al. A comprehensive review of $\mathrm{ZnO}$ materials and devices. J. Appl. Phys. 98, 041301 (2005).

96. Song, Z. et al. Characterization of optical properties of $\mathrm{ZnO}$ nanoparticles for quantitative imaging of transdermal transport. Biomed. Opt. Express 2, 3321 (2011).

97. Reddy, K. M. et al. Selective toxicity of zinc oxide nanoparticles to prokaryotic and eukaryotic systems. Appl. Phys. Lett. 90, 2139021-2139023 (2007).

98. Dhas, S. P., Shiny, P. J., Khan, S., Mukherjee, A. \& Chandrasekaran, N. Toxic behavior of silver and zinc oxide nanoparticles on environmental microorganisms. J. Basic Microbiol. 916-927 (2013). doi:10.1002/jobm.201200316

99. Zhang, Y. et al. Immune responses during single and repeated murine endotracheal exposures of zinc oxide nanoparticles. NanoImpact 7, 54-65 (2017).

100. Taccola, L. et al. Zinc oxide nanoparticles as selective killers of proliferating cells. Int. J. Nanomedicine 6, 1129-40 (2011).

101. Sawai, J. et al. Hydrogen peroxide as an antibacterial factor in zinc oxide powder slurry. J. Ferment. Bioeng. 86, 521-522 (1998). 
102. Applerot, G. et al. Enhanced Antibacterial Activity of Nanocrystalline ZnO Due to Increased ROS-Mediated Cell Injury. Adv. Funct. Mater. 19, 842-852 (2009).

103. Akhtar, M. J. et al. Zinc oxide nanoparticles selectively induce apoptosis in human cancer cells through reactive oxygen species. Int. J. Nanomedicine 7, 845-57 (2012).

104. Brayner, R. et al. Toxicological impact studies based on Escherichia coli bacteria in ultrafine $\mathrm{ZnO}$ nanoparticles colloidal medium. Nano Lett. 6, 866-870 (2006).

105. Zhang, L., Ding, Y., Povey, M. \& York, D. ZnO nanofluids - A potential antibacterial agent. Prog. Nat. Sci. 18, 939-944 (2008).

106. Mudunkotuwa, I. A., Rupasinghe, T., Wu, C.-M. \& Grassian, V. H. Dissolution of ZnO Nanoparticles at Circumneutral pH: A Study of Size Effects in the Presence and Absence of Citric Acid. Langmuir 28, 396-403 (2012).

107. Lv, J. et al. Dissolution and Microstructural Transformation of $\mathrm{ZnO}$ Nanoparticles under the Influence of Phosphate. Environ. Sci. Technol. 46, 7215-7221 (2012).

108. Archana, J., Navaneethan, M. \& Hayakawa, Y. Morphological transformation of $\mathrm{ZnO}$ nanoparticle to nanorods via solid-solid interaction at high temperature annealing and functional properties. Scr. Mater. 113, 163-166 (2016).

109. Dahle, J., Arai, Y., Dahle, J. T. \& Arai, Y. Environmental Geochemistry of Cerium: Applications and Toxicology of Cerium Oxide Nanoparticles. Int. J. Environ. Res. Public Health 12, 1253-1278 (2015).

110. Hayes, S. A., Yu, P., O’Keefe, T. J., O’Keefe, M. J. \& Stoffer, J. O. The Phase Stability of Cerium Species in Aqueous Systems. J. Electrochem. Soc. 149, C623 (2002).

111. Zholobak, N. M. et al. UV-shielding property, photocatalytic activity and photocytotoxicity of ceria colloid solutions. J. Photochem. Photobiol. B Biol. 102, 32-38 (2011).

112. Pirmohamed, T. et al. Nanoceria exhibit redox state-dependent catalase mimetic activity. Chem. Commun. 46, 2736 (2010).

113. Deshpande, S., Patil, S., Kuchibhatla, S. V. \& Seal, S. Size dependency variation in lattice parameter and valency states in nanocrystalline cerium oxide. Appl. Phys. Lett. 87, 133113 (2005).

114. Molinari, M., Parker, S. C., Sayle, D. C. \& Islam, M. S. Water Adsorption and Its Effect on the Stability of Low Index Stoichiometric and Reduced Surfaces of Ceria. J. Phys. Chem. C 116, 7073-7082 (2012).

115. Karakoti, A. S. et al. PEGylated Nanoceria as Radical Scavenger with Tunable Redox Chemistry. J. Am. Chem. Soc. 131, 14144-14145 (2009).

116. Korsvik, C., Patil, S., Seal, S. \& Self, W. T. Superoxide dismutase mimetic properties exhibited by vacancy engineered ceria nanoparticles. Chem. Commun. 
1056 (2007). doi:10.1039/b615134e

117. Ivanov, V. K. et al. Inactivation of the nitroxyl radical by ceria nanoparticles. Dokl. Chem. 430, 43-46 (2010).

118. Taylor, N. S. et al. Molecular toxicity of cerium oxide nanoparticles to the freshwater alga Chlamydomonas reinhardtii is associated with supraenvironmental exposure concentrations. Nanotoxicology 1-10 (2015). doi:10.3109/17435390.2014.1002868

119. Pulido-Reyes, G. et al. Untangling the biological effects of cerium oxide nanoparticles: the role of surface valence states. Sci. Rep. 5, 15613 (2015).

120. Chen, J., Patil, S., Seal, S. \& McGinnis, J. F. Nanoceria Particles Prevent ROIInduced Blindness. in 53-59 (Springer, New York, NY, 2008). doi:10.1007/978-0387-74904-4_5

121. Rzigalinski, B. A. n et al. Cerium oxide nanoparticles increase the lifespan of cultured brain cells and protect against free radical and mechanical trauma. FASEB J. 17, 37724 (2003).

122. Karakoti, A., Singh, S., Dowding, J. M., Seal, S. \& Self, W. T. Redox-active radical scavenging nanomaterials. Chem. Soc. Rev. 39, 4422 (2010).

123. García, A. et al. Acute toxicity of cerium oxide, titanium oxide and iron oxide nanoparticles using standardized tests. Desalination 269, 136-141 (2011).

124. Park, E.-J., Choi, J., Park, Y.-K. \& Park, K. Oxidative stress induced by cerium oxide nanoparticles in cultured BEAS-2B cells. Toxicology 245, 90-100 (2008).

125. Nalabotu, S. K. et al. Intratracheal instillation of cerium oxide nanoparticles induces hepatic toxicity in male Sprague-Dawley rats. Int. J. Nanomedicine 6 , 2327-35 (2011).

126. Zhang, H. et al. Nano-CeO 2 Exhibits Adverse Effects at Environmental Relevant Concentrations. Environ. Sci. Technol. 45, 3725-3730 (2011).

127. Pulido-Reyes, G. et al. Internalization and toxicological mechanisms of uncoated and PVP-coated cerium oxide nanoparticles in the freshwater alga Chlamydomonas reinhardtii. Environ. Sci. Nano 6, 1959-1972 (2019).

128. Mittal, S. \& Pandey, A. K. Cerium oxide nanoparticles induced toxicity in human lung cells: role of ROS mediated DNA damage and apoptosis. Biomed Res. Int. 2014, 891934 (2014).

129. Schwabe, F. et al. Dissolution and transformation of cerium oxide nanoparticles in plant growth media. J. Nanoparticle Res. 16, 2668 (2014).

130. Dale, J. G., Cox, S. S., Vance, M. E., Marr, L. C. \& Hochella, M. F. Transformation of Cerium Oxide Nanoparticles from a Diesel Fuel Additive during Combustion in a Diesel Engine. Environ. Sci. Technol. 51, 1973-1980 (2017). 


\section{Chapter 2: Analysis Techniques}

This chapter will focus on explaining the working principles of some of the major analytical techniques used in this research. These techniques were specifically chosen to provide the required data to help achieve the research objectives.

\subsection{Transmission Electron Microscope (TEM)}

The TEM was chosen to obtain images of the nanoparticles in this study. The leap into electron microscopy in 1931 helped overcome the resolution limits of light microscopy ${ }^{1}$. The use of electrons was possible due to Louis de Broglie's hypothesis that proposes all matter to exhibit wave-like property. The earlier electron microscopes were scanning electron microscopes (SEM), where the reflection of electrons on the surface of samples was imaged. Later, increasing the accelerating voltage of the electrons caused it to penetrate the samples at high speeds. The transmission of electrons through the sample lead to the making of a TEM. A modern TEM will be able to provide a practical resolution of $0.2 \mathrm{~nm}^{2}$, due to limitations from aberrations caused by the imperfections on electromagnetic lenses and ununiform nature of the electron beam. Attempts to combat aberrations by increasing operational voltage caused damage to fragile samples ${ }^{3}$. 


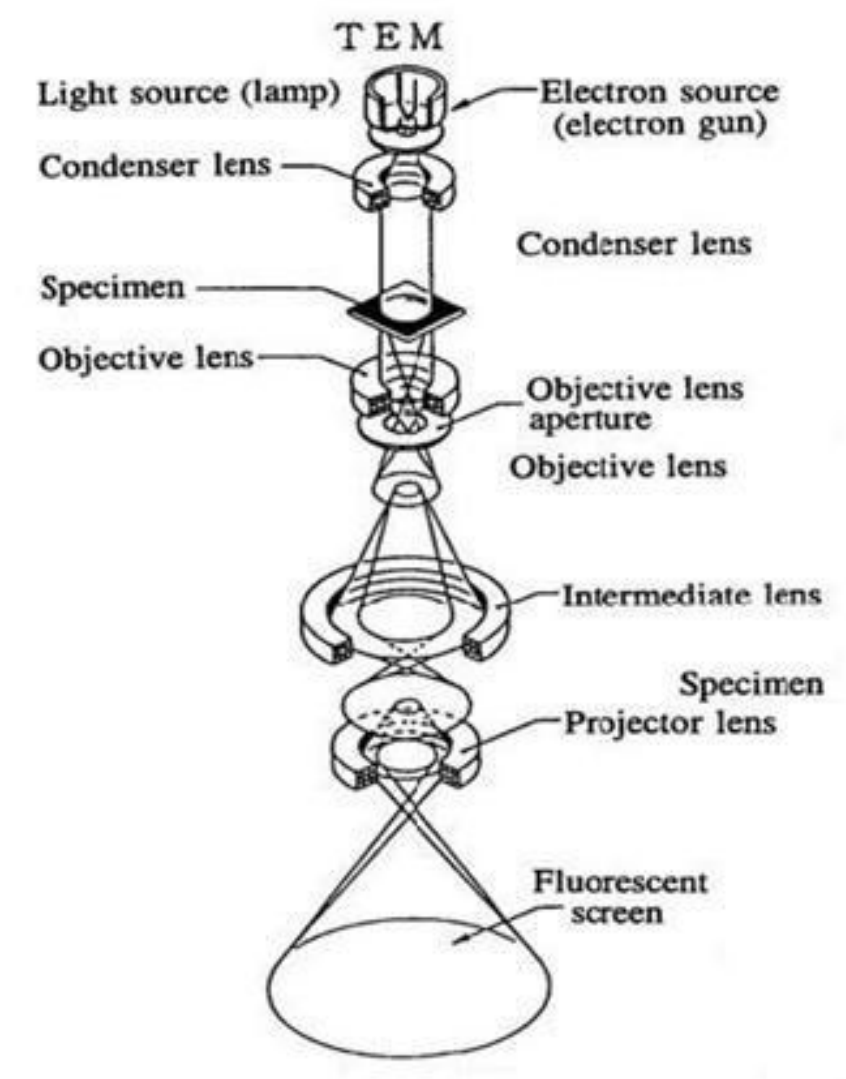

Figure 2-1: Diagram of TEM components ${ }^{4}$ used with permission from North Carolina State University.

A diagram of the various parts of the TEM can be seen in Figure 2-1 above. The first part of the TEM is the electron gun. Electrons are produced most commonly by heating a tungsten filament in the electron gun. The process of releasing electron by heat is called thermionic emission ${ }^{5,6}$. The electrons released are accelerated at high voltage $(100-400 \mathrm{kV})$ to achieve high electron velocity that would yield a sharper, highresolution image. ${ }^{7}$ The electrons then pass through the condenser lens that helps focus the electron beam onto the specimen. Typically, electron microscopes have two condenser lenses. The first lens will have a fixed aperture and the second lens will have a variable aperture. The variable aperture of the lens helps with obtaining images at different 
magnifications. Smaller aperture size is used to concentrate electrons into a small focal point on the specimen, which will produce a high magnification image ${ }^{8}$.

In order to produce an image on the TEM, some electrons will have to pass through the sample. The sample has to be very thin $(<100 \mathrm{~nm})$ for the electrons to pass through. It is a challenge to deposit a thin sample, especially when dealing with organisms. Moreover, samples must be completely dehydrated, and heat resistant to maintain its structure as being bombarded by high-speed electrons produces much heat. The sample inserted in the TEM also has to be stable in a vacuum. The vacuum environment in the TEM reduces noise that can be caused by electrons scattered by air particles. The electrons will then scatter as it encounters the various atoms in the sample. The sample is usually prepared on a carbon film within a mesh. The carbon atoms are small enough that the electrons will pass through with minimum interference ${ }^{9,10}$.

Electrons that are scattered by the sample passes through the objective lens to form the first intermediate image. The next lens that the electron pass-through is the intermediate lens. The intermediate lens is used to focus on a specific diffraction pattern produced by the samples. Images produced by strongly diffracting the electrons that pass through crystalline structures are called dark-field images ${ }^{11}$. Finally, electrons pass through the projector lens to be further magnified and projected onto the image sensor ${ }^{8}$.

Electrons projected from a TEM can be observed in real-time on a fluorescent screen. The image on a fluorescent screen is generated by the excitation of a phosphor caused by the collision of electrons. The phosphor that is excited exhibits a green color. The fluorescent screen also acts as a shutter that keeps the beam from entering the 
charge-coupled device (CCD) camera sensor, which is used to produce a digital image $^{12,13}$.

The TEM images in this study were obtained using FEI Tecnai G2 TEM (Thermo Fisher Scientific, Waltham, MA, USA) at $200 \mathrm{kV}$. Samples were prepared on copper mech TEM grids, CF300-CU, purchased from Electron Microscopy Sciences, Hatfield, PA, USA. Due to the TEM giving an image of a very small window of the sample, multiple replicates are were analyzed. Moreover, on each replicate, two TEM grids were prepared. At least 5 images were taken from different grid locations on the same TEM grid.

The TEM images provided evidence of a morphological transformation to the nanoparticles tested. The aggregation and agglomeration of nanoparticles were determined through the visual analysis of TEM images. The nanoparticles were determined to be aggregated when the nanoparticles are clustered together, the distinction between individual particles is clearly visible, and the size of individual particles remained unchanged. The nanoparticles were determined to be agglomerated when larger and pristine sized particles were observed. The samples prepared for TEM imaging needs to be diluted to have only a thin deposition of nanoparticles as highly dense clusters of nanoparticles formed during the drying process can be inaccurately analyzed as a large agglomeration. The transformations that had been presented in this research had been found to be consistent on the multiple replicates and on the various grid locations that were imaged. 


\subsection{Energy-Dispersive X-ray Spectroscopy (EDS)}

EDS was chosen to analyze the elemental composition of the nanoparticles and the nanoparticle matrix analyzed in this study. EDS analysis can be performed with high accuracy on specific locations on a sample with a spatial resolution of $0.03 \mathrm{~nm}$. The accuracy of EDS helps analyze distribution elements at various points in a sample. EDS spectroscopy is usually built into other instruments like TEM and SEM, where the elemental composition can help confirm the location of the desired sample. Furthermore, EDS works by exciting the surface of the sample with a high energy electron beam, which already exists in electron microscopes ${ }^{14}$. The electrons hitting the sample causes the emission of a specific wavelength of X-Ray dependent upon the atomic structure of the elements in the sample ${ }^{15}$. 


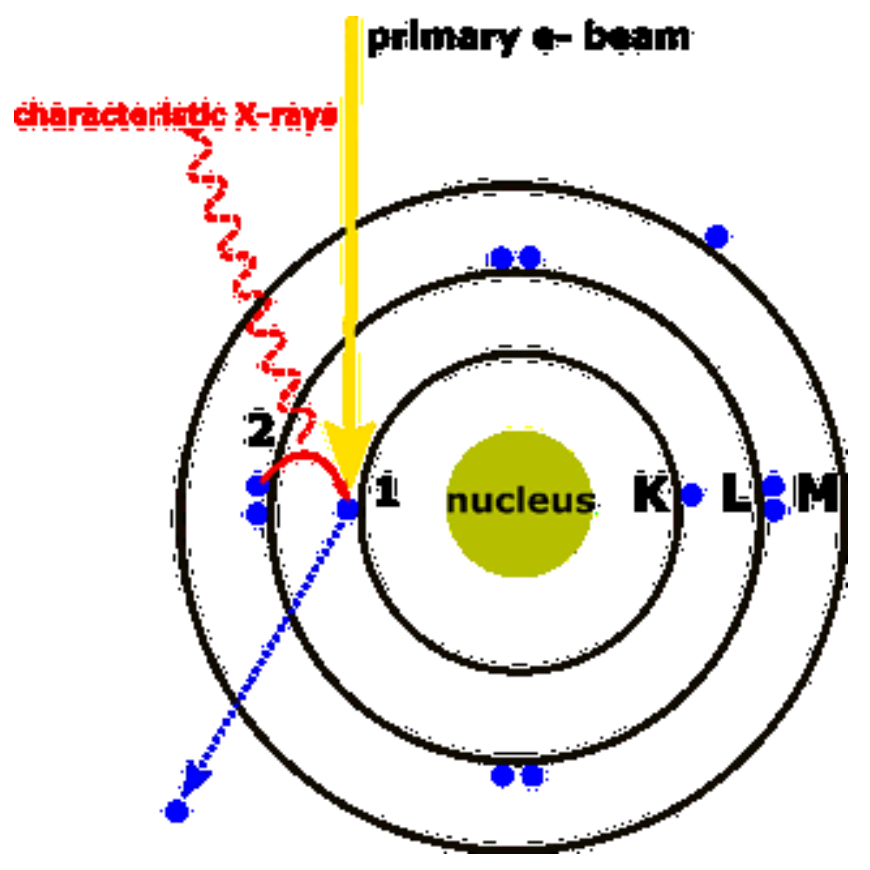

Figure 2-2: The process of generation of X-rays. (1) The electron beam knocks off one of the electrons in the atom, leaving a void. (2) The void is filled by an electron from a higher energy shell, causing a very specific release of energy ${ }^{16}$. This image was used with permission from ThermoFisher Scientific.

Elemental identification with EDS is very accurate due to the uniqueness of every atom having a specific number of electrons at fixed positions within the atom. The electrons are positioned in shells with different energies. As shown in Figure 2-2, the Xray detected by EDS is generated from an atom by first absorbing the energy from the electron beam to displace an electron to a higher energy level or out of the atom. The empty void left by the electron is later filled by a different electron from a higher energy level, which causes the release of energy as an X-ray ${ }^{16}$. The various energies of X-rays released are detected by an energy dispersive detector. Software is used to count the number of X-rays received (Y-axis) at specific energy levels (X-axis). Being able to 
identify the elements and count the signals received make EDS a qualitative and quantitative analysis ${ }^{17}$.

The EDS of the samples in this research was obtained via an attachment to the TEM, Aztec EDS from Oxford X-ray detection systems (Oxford Instruments, Tubney Woods, Abingdon, UK). For the purposes of this, EDS was used as a qualitative method only, as analyzing a complex matrix with varying thickness will provide inconsistent quantitative results ${ }^{18}$. The channeling effect, where individual atoms act as miniature lenses to the electron beam, will affect the EDS signal count when the thickness of the sample varies ${ }^{19}$. However, channeling is beneficial in providing a higher signal count when focused on a single point of the sample ${ }^{18}$.

EDS analysis were performed on all the TEM images obtained in this research. The EDS analysis showed the various elements that exist in the same location as the nanoparticles. The conclusion is drawn on the association of the element found by EDS with the nanoparticle when the result is consistent throughout the various replicated and grid locations. The background of the nanoparticles was also analyzed to confirm that the elements found are not part of the surrounding matrix.

\subsection{Inductively coupled plasma, mass spectrometry (ICP-MS)}

A mass spectrometer is an instrument that measures the mass to charge ratio and the relative abundance of an ion ${ }^{20}$. Molecules are first bombarded by a stream of highenergy electrons, which ionizes some of the molecules that are then accelerated in an 
electric field. Then, these accelerated ions are separated according to their mass-to-charge ratios in an electric or magnetic field. The ions that have a specific mass-to-charge ratio are recognized by a sensor, which will also count the number of ions hitting $\mathrm{it}^{21}$.

In an ICP-MS, samples are ionized by an inductively coupled plasma. The sample introduction system consists of the nebulizer, spray chamber, and ICP torch. Ion generation takes place in a high-temperature plasma $(>10000 \mathrm{~K})$ that atomizes the sample to creates atomic and small polyatomic ions ${ }^{22,23}$.

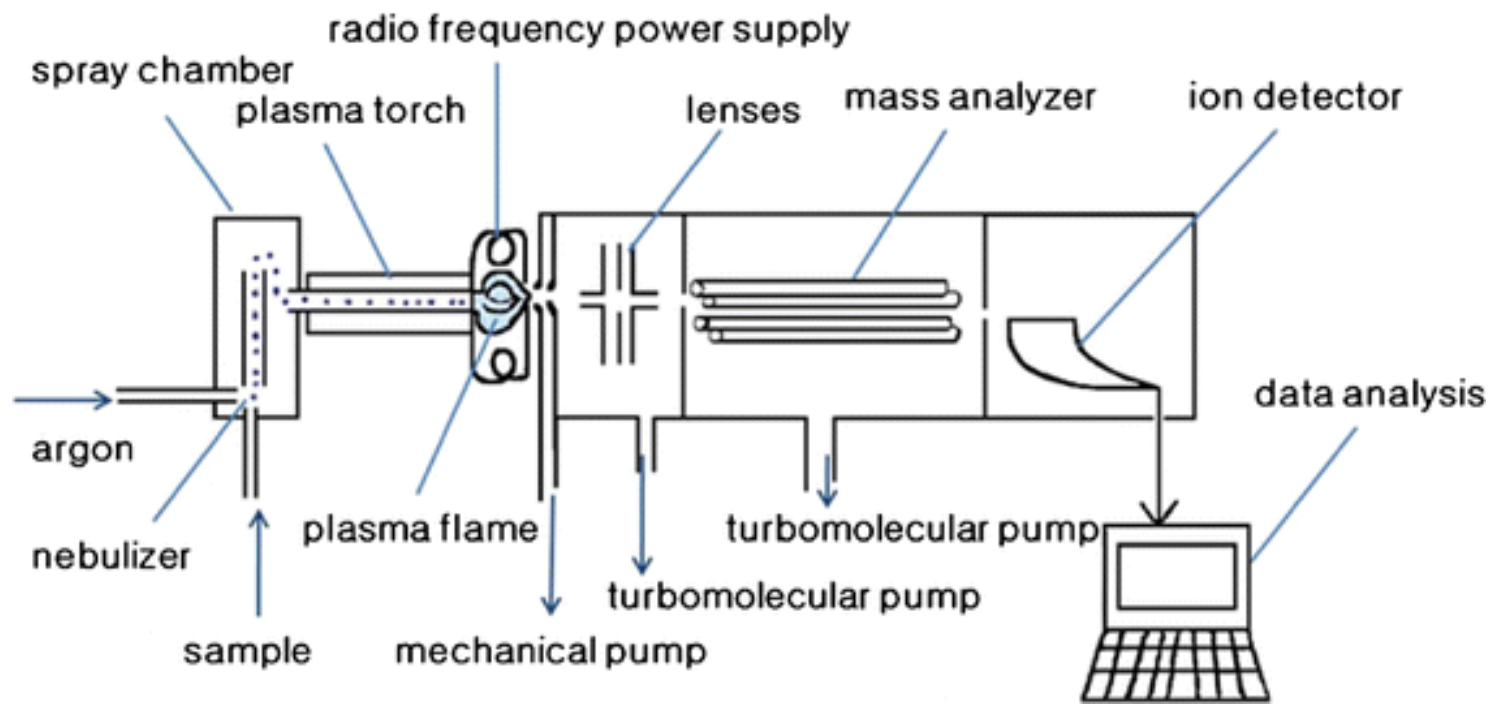

Figure 2-3: Schematic of the ICP-MS system ${ }^{24}$ used with permission from Springer Vienna.

Figure 2-3 above shows the operational schematics of an ICP-MS. The sample is pumped from sample vial into a nebulizer where it is mixed with argon gas to form an aerosol. The fine aerosol is swept into the central channel of the plasma torch. As they travel through the high-temperature argon plasma, the aerosol droplets are dried, 
decomposed, atomized, and finally ionized, producing a rich source of positively charged ions. The ions are extracted from the plasma into the interface through sampling orifice and then through the skimmer cone and to the high vacuum region containing the ion lenses and mass analyzer. Electrostatic lenses focus the ion beam and separate the positive ions from unwanted neutral species. The ion detector detects the ion and the data can be later analyzed ${ }^{24}$.

ICP-MS combined with a powerful data analysis software, is a widely used technique in nanoparticle characterization ${ }^{25-27}$. A variant of the ICP-MS, called the single-particle(SP)-ICP-MS, is unique in its ability to give information on NP size, size distribution, elemental composition, and number concentration in just one quick analysis ${ }^{28,29}$. However, the shortcoming of this technique techniques is the presence of a dissolved analyte that may produce a continuous signal that will mask the signals produced by individual particles entering the plasma ${ }^{29}$.

In this research, ICP-MS was used to measure the concentration of metal nanoparticles in commercial products as well as the number of nanoparticles leaching out when the products are put through simulated day-to-day use scenarios and incubated in wastewater. Attempts were made to size and count ZnO NPs using SP-ICP-MS; however, the high dissolution rate of $\mathrm{ZnO}$ NPs made a high background signal that masked the signals from the nanoparticles. 


\subsection{X-Ray Photoelectron Spectrometer (XPS)}

In this study, XPS was used to detect the change in the oxidation state of the elements comprising the nanoparticles. X-ray photoelectron spectroscopy (XPS) is a quantitative spectroscopic technique that is used for materials studies such as nanoparticles ${ }^{30}$ to determine the elemental composition of the surface (top $0-10 \mathrm{~nm}$ ), the empirical formula of pure materials, and the chemical or electronic state of each element in the surface ${ }^{31}$. A material under investigation is irradiated with a beam of low energy Xrays, causing electrons (a.k.a. photoelectrons) to escape from the top layer of the material being analyzed ${ }^{32,33}$, as shown in Figure 2-4 . The kinetic energy (KE) of the photoemitted electron is calculated to identify the elements in the analyzed material ${ }^{31}$.

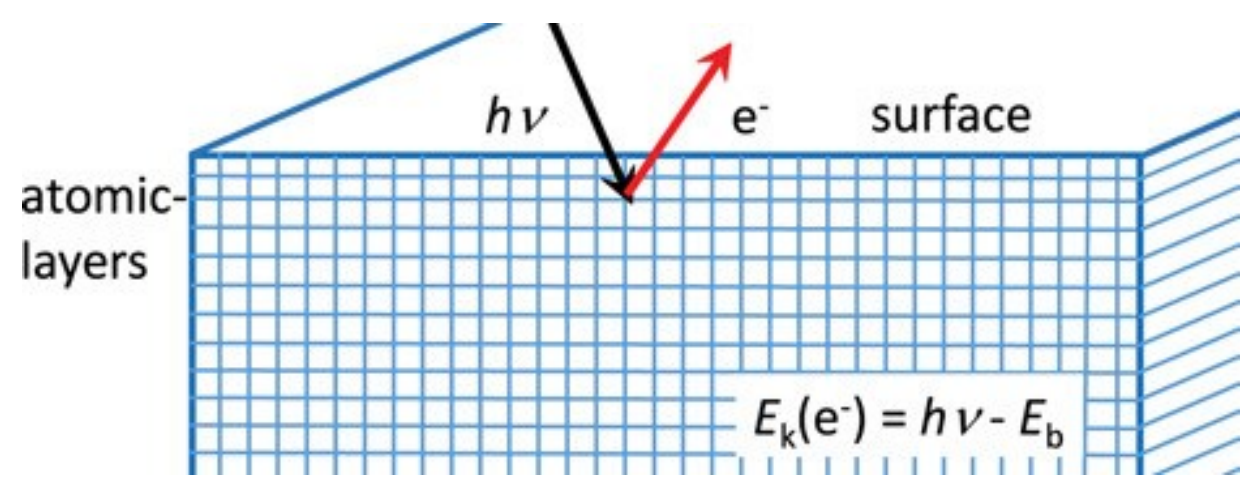

Figure 2-4: Photoelectron release upon irradiation with an X-ray beam in $\mathrm{XPS}^{33}$ used with permission from Elsevier.

XPS is a very sensitive and non-invasive technique that can detect all the elements with atomic number 3 and above ( $\mathrm{H}$ and $\mathrm{He}$ cannot be identified as it only has one shell of electrons $)^{34}$. The energies and intensities of the photoelectron peaks enable the identification and quantification of all surface elements ${ }^{35}$. Peaks appear in the XPS 
spectrum from atoms emitting electrons of characteristic kinetic energy $(\mathrm{eV})$ plotted against the number of counts ${ }^{30,33}$.

The ability of XPS to provide information on oxidation state changes is a piece of important information for this thesis ${ }^{36}$. The change in the oxidation state is used to support the occurrence of a compositional transformation within the nanoparticles analyzed. The transformation of the nanoparticle is only detected on the surface, as the Xrays do not penetrate the nanoparticles. Any oxidation state changes within the nanoparticle can not be deduced by only XPS ${ }^{37}$. 


\subsection{References}

1. Introduction to Electron Microscopy - History of Electron Microscopy. (2018). Available at: https://www.fei.com/introduction-to-electron-microscopy/history/. (Accessed: 10th November 2019)

2. Otten, M. T. \& Coene, W. M. J. High-resolution imaging on a field emission TEM. Ultramicroscopy 48, 77-91 (1993).

3. Ke, X., Bittencourt, C. \& Van Tendeloo, G. Possibilities and limitations of advanced transmission electron microscopy for carbon-based nanomaterials. Beilstein J. Nanotechnol. 6, 1541-1557 (2015).

4. NC State University. Transmission Electron Microscopy Laboratories Analytical Instrument Facility JEM-2000FX Scanning Transmission Electron Microscope (S/TEM) Basic TEM Operation Procedure Panel assignment for Left panels and Right Panels. (2013).

5. Johnson, T. L. Thermionic Emission vs Field Emission for Non-Relativistic Electron Guns. (2019).

6. Herring, C. \& Nichols, M. H. Thermionic Emission. Rev. Mod. Phys. 21, 185-270 (1949).

7. Egerton, R. F. Choice of operating voltage for a transmission electron microscope. Ultramicroscopy 145, 85-93 (2014).

8. Wang, C. Lenses and Apertures of A TEM. EMA 6518 Course Presentation (Florida International University).

9. Lambrecht, E. et al. Transmission electron microscopy sample preparation protocols for the ultrastructural study of cysts of free-living protozoa. Biotechniques 58, (2015).

10. Mielańczyk, Ł., Matysiak, N., Klymenko, O. \& Wojnicz, R. Transmission Electron Microscopy of Biological Samples. in The Transmission Electron Microscope Theory and Applications (InTech, 2015).

11. Tang, C. Y. \& Yang, Z. Transmission Electron Microscopy (TEM). in Membrane Characterization 145-159 (Elsevier, 2017).

12. Fan, G. Y. \& Ellisman, M. H. Digital imaging in transmission electron microscopy. J. Microsc. 200, 1-13 (2000).

13. Contarato, D., Denes, P., Doering, D., Joseph, J. \& Krieger, B. High Speed, Radiation Hard CMOS Pixel Sensors for Transmission Electron Microscopy. Phys. Procedia 37, 1504-1510 (2012). 
14. Mehrban, N. \& Bowen, J. Monitoring biomineralization of biomaterials in vivo. in Monitoring and Evaluation of Biomaterials and their Performance In Vivo 81-110 (Elsevier, 2017).

15. Ebnesajjad, S. \& Ebnesajjad, C. Surface Treatment of Materials for Adhesive Bonding. (Elsevier Science\& Technology Books, 2013).

16. Nanakoudis, A. EDX Analysis with a Scanning Electron Microscope (SEM): How Does it Work? (2019). Available at: https://blog.phenom-world.com/edx-analysissem. (Accessed: 1st November 2019)

17. Plant-Mediated Green Synthesis of Nanostructures: Mechanisms, Characterization, and Applications. Interface Sci. Technol. 28, 199-322 (2019).

18. MacArthur, K. E., Brown, H. G., Findlay, S. D. \& Allen, L. J. Probing the effect of electron channelling on atomic resolution energy dispersive X-ray quantification. Ultramicroscopy 182, 264-275 (2017).

19. Lugg, N. R., Kothleitner, G., Shibata, N. \& Ikuhara, Y. On the quantitativeness of EDS STEM. Ultramicroscopy 151, 150-159 (2015).

20. Ekman, R. Mass spectrometry: instrumentation, interpretation, and applications. (John Wiley \& Sons, 2009).

21. Pavia, D. L., Lampman, G. M., Kriz, G. S. \& Vyvyan, J. Introduction to spectroscopy. (Brooks/Cole, Cengage Learning, 2009).

22. Ammann, A. A. Inductively coupled plasma mass spectrometry (ICP MS): a versatile tool. J. Mass Spectrom. 42, 419-427 (2007).

23. Bogaerts, A. \& Aghaei, M. Inductively coupled plasma-mass spectrometry: insights through computer modeling. J. Anal. At. Spectrom. 32, 233-261 (2017).

24. Ha, Y., Tsay, O. G. \& Churchill, D. G. A tutorial and mini-review of the ICP-MS technique for determinations of transition metal ion and main group element concentration in the neurodegenerative and brain sciences. Monatshefte für Chemie - Chem. Mon. 142, 385-398 (2011).

25. Hu, J., Deng, D., Liu, R. \& Lv, Y. Single nanoparticle analysis by ICPMS: a potential tool for bioassay. J. Anal. At. Spectrom. 33, 57-67 (2018).

26. Correia, M., Uusimäki, T., Philippe, A. \& Loeschner, K. Challenges in Determining the Size Distribution of Nanoparticles in Consumer Products by Asymmetric Flow Field-Flow Fractionation Coupled to Inductively Coupled Plasma-Mass Spectrometry: The Example of $\mathrm{Al} 2 \mathrm{O} 3, \mathrm{TiO} 2$, and $\mathrm{SiO} 2$ Nanoparticles in Toothpaste. Separations 5, 56 (2018).

27. Stephan, C. Single Particle Inductively Coupled Plasma Mass Spectrometry : 
Understanding How and Why Authors. Perkin Elmer 1-5 (2014).

28. Wilbur, S., Yamanaka, M. \& Sannac, S. Characterization of nanoparticles in aqueous samples by ICP-MS White paper. (2015).

29. Schwertfeger, D. M., Velicogna, J. R., Jesmer, A. H., Scroggins, R. P. \& Princz, J. I. Single Particle-Inductively Coupled Plasma Mass Spectroscopy Analysis of Metallic Nanoparticles in Environmental Samples with Large Dissolved Analyte Fractions. Anal. Chem. 88, 9908-9914 (2016).

30. Baer, D. R., Gaspar, D. J., Nachimuthu, P., Techane, S. D. \& Castner, D. G. Application of surface chemical analysis tools for characterization of nanoparticles. Anal. Bioanal. Chem. 396, 983-1002 (2010).

31. Lefebvre, J., Galli, F., Bianchi, C. L., Patience, G. S. \& Boffito, D. C. Experimental methods in chemical engineering: X-ray photoelectron spectroscopyXPS. Can. J. Chem. Eng. 97, 2588-2593 (2019).

32. Gorgoi, M., Schäfers, F., Svensson, S. \& Mårtensson, N. Relative sub-shell photoionization cross-sections of nickel metal determined by hard X-ray high kinetic energy photoemission. J. Electron Spectros. Relat. Phenomena 190, 153158 (2013).

33. Fadley, C. S. X-ray photoelectron spectroscopy: Progress and perspectives. $J$. Electron Spectros. Relat. Phenomena 178-179, 2-32 (2010).

34. Stojilovic, N. Why Can't We See Hydrogen in X-ray Photoelectron Spectroscopy? J. Chem. Educ. 89, 1331-1332 (2012).

35. Smith, G. C. Quantification of Data from Homogeneous Materials. in Surface Analysis by Electron Spectroscopy 53-73 (Springer US, 1994).

36. Bêche, E., Charvin, P., Perarnau, D., Abanades, S. \& Flamant, G. Ce 3d XPS investigation of cerium oxides and mixed cerium oxide (CexTiyOz). Surf. Interface Anal. 40, 264-267 (2008).

37. Baer, D. R., Gaspar, D. J., Nachimuthu, P., Techane, S. D., \& Castner, D. G.. Application of surface chemical analysis tools for characterization of nanoparticles. Anal. and bioanal. chem. 396(3), 983-1002 (2010). 


\section{Chapter 3: Morphological transformation of silver nanoparticles from commercial products: modeling from product incorporation, weathering through use scenarios, and leaching into wastewater}

Selvan Mohan, Juliska Princz, Banu Ormeci, Maria C. DeRosa

\subsection{Abstract:}

There is increasing interest in the environmental fate and effects of engineered nanomaterials due to their ubiquitous use in consumer products. In particular, given the mounting evidence that dramatic transformations can occur to a nanomaterial throughout its product lifecycle, the appropriateness of using pristine nanomaterials in environmental testing is being questioned. Using a combination of transmission electron microscopy (TEM), energy dispersive X-ray spectroscopy (EDS), X-ray photoelectron spectroscopy (XPS), and inductively coupled plasma-mass spectrometry (ICP-MS), this work examines the morphological and compositional effects of conditions mimicking a typical lifecycle of a nano-enabled product, from the production of the silver nanoparticle (AgNP)-laden textiles, through its use, laundering, and then finally, its leaching and incubation in the wastewater collection system. These simulated weathering conditions showed evidence for the transformation of $\mathrm{AgNPs}$ into $\mathrm{AgCl}$ and $\mathrm{Ag}_{2} \mathrm{~S}$. Incubation in raw wastewater had the most dramatic effect on the AgNPs in terms of transformation, no matter what initial weathering was applied to the NPs prior to incubation. However, despite extensive transformation noted, AgNPs were still present within all the samples after the use scenarios. 


\subsection{Introduction}

The unique properties of nanoparticles (NPs) make them attractive additives in a variety of commercial products. With the heightened interest in this area of research has come a plethora of novel nanomaterials of varying composition and morphology, many with attractive possible applications ${ }^{1,2}$. Of the over 1600 products claiming to contain nanomaterials, 383 of them explicitly declare the incorporation of silver ${ }^{3}$. Although AgNPs are applied to many different commercial products worldwide, the majority of them are used in textiles for antimicrobial purposes ${ }^{4-7}$. The ubiquity of AgNP-laden products in the marketplace sparks the question of the eventual environmental fate and effects of these materials.

Studies on the environmental impact of nanoparticles are typically carried out using the material in pristine form and vary widely in size $(10-50 \mathrm{~nm})$ and coatings depending on the availability and ease of synthesis of the nanoparticles ${ }^{8-14}$. Numerous studies have established the potential ecotoxic effects of AgNPs. They inhibit the photosynthesis of algae, increase the mortality rate of aquatic zebrafish embryos, and disrupt microbial colonies in soil ${ }^{12-19}$. While an understanding of the potential impact of pristine nanoparticles on the environment is essential, particularly in simulating accidental spill scenarios, these results might not be universally applicable to all exposure situations, given the transformations that NPs could undergo throughout its life cycle ${ }^{22}$. Transformations can include the agglomeration or aggregation of the nanoparticle or a chemical/surface transformation triggered by exposure to other agents within the product or exposed to the product. Although often used interchangeably, aggregation is defined as the loose assembly of particles and agglomeration is when the more permanent assembly 
of the particles into tight clusters causing partial fusion ${ }^{23}$. Several studies have already established that transformations to $\mathrm{AgCl}$ or $\mathrm{Ag}_{2} \mathrm{~S}$ are possible for $\mathrm{AgNPs}^{24}$. This transformation has been previously described as oxidative dissolution by chlorine and sulfidation, respectively ${ }^{25,26}$. However, the presence of chloride, nitrate, selenide, and sulfide have also been known to lead to AgNPs transformations ${ }^{27-29}$. Indeed, analyzing transformed NPs and subsequent byproducts released from the use of products containing NPs will inform environmental fate and toxicological studies, as well as risk assessment initiatives ${ }^{30}$.

With regards to Ag, studies have shown that AgNPs are released from AgNPladen fabric using only water washes ${ }^{31,32}$. Treatments with sweat and detergent have also been shown to trigger the release of NPs ${ }^{33-35}$. The composition, $\mathrm{pH}$, and ionic strength of these solutions will contribute to the variability in the form of Ag extracted ${ }^{24}$. As the fabrics are exposed to these conditions (e.g., washing, sweat, etc.), the potential is high that the leachates from the consumer product, containing transformed AgNPs, will eventually end up in the wastewater treatment process ${ }^{36}$. However, even before reaching the wastewater treatment facility, incubation in the wastewater collection system could further transform the nanomaterials. Pristine nanoparticles are known to disrupt microbial colonies, biofilms, and alter the chemical properties of the wastewater, hindering the effectiveness of the wastewater treatment ${ }^{37-39}$. Further transformation of these AgNPs can be expected through the wastewater treatment, as well as with environmental weathering ${ }^{40-42}$. Finally, sludge from wastewater is treated for use in agricultural applications ${ }^{43}$. The transformation of AgNPs to $\mathrm{Ag}_{2} \mathrm{~S}$ when added directly to the sludge, 
has been reported ${ }^{44}$. $\mathrm{Ag}_{2} \mathrm{~S}$ nanoparticles of 5-10 $\mathrm{nm}$ have also been independently detected in sewage sludge ${ }^{45}$

A clearer understanding of the physical and chemical changes that take place throughout the lifecycle of a nano-enabled product would help inform toxicological studies and improve our risk assessment for nanomaterials. In this paper, the morphology and composition of AgNPs, when embedded into fabric, are compared following a variety of treatments meant to mimic several stages in the lifecycle of the nanomaterial, from initial product incorporation to use and washing, to right before the wastewater treatment process (Figure 3-1).

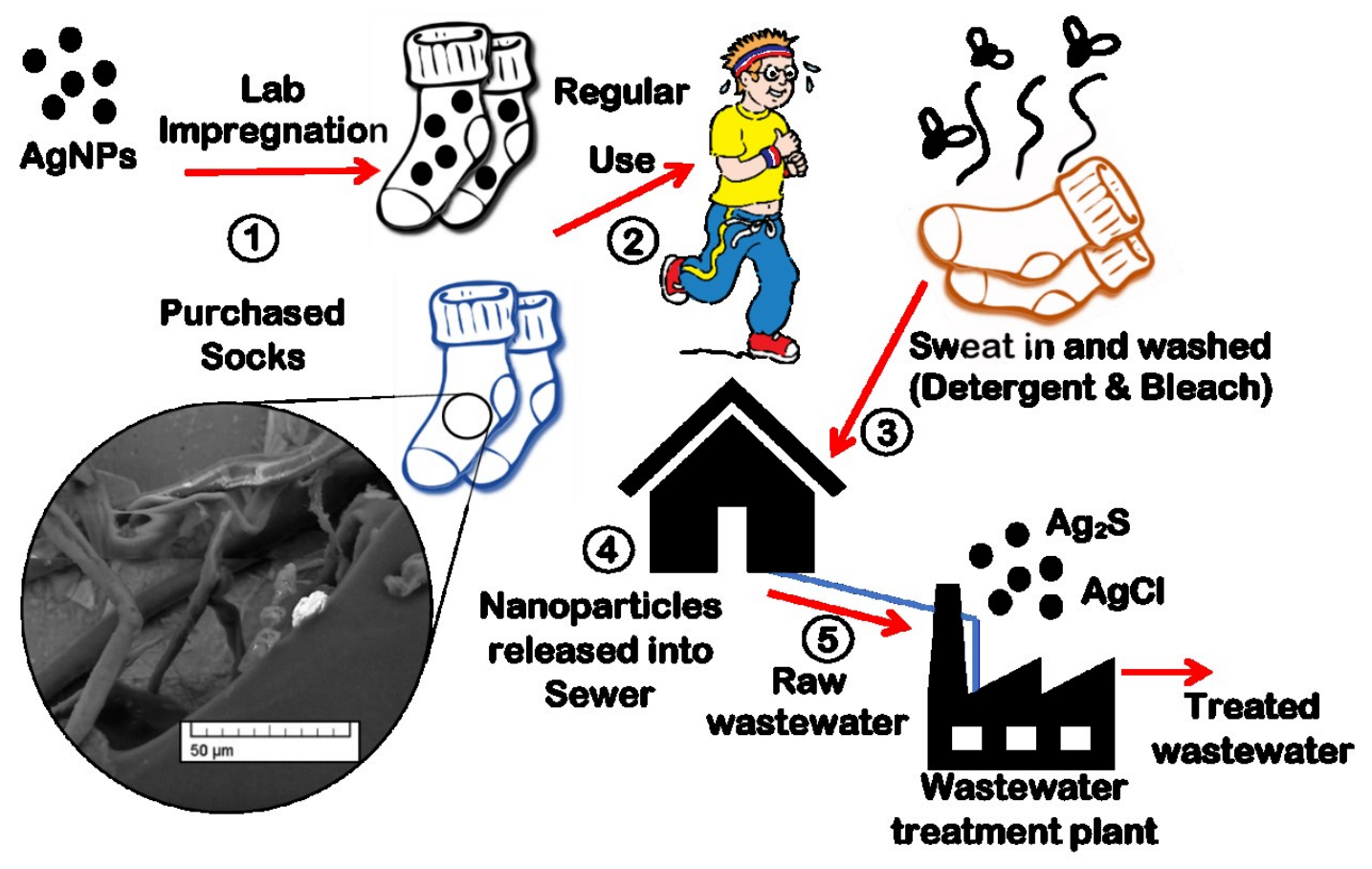

Figure 3-1. Overview of the path of potential transformations for AgNPs studied in this paper. 


\subsection{Materials and Methods}

Pristine AgNPs, $25 \pm 3 \mathrm{~nm}$ Econix Silver Nanospheres (5 mg/mL concentration, $23 \mathrm{mV}$ zeta potential) were purchased from Nanocomposix (Figure 3-2). Socks with AgNPs (Sports Socks) were purchased from Nanosilver (commercial socks). White Hanes sport cut socks, Clorox ${ }^{\circledR}$ bleach and Tide ${ }^{\circledR}$ Original laundry detergent were used as received. Raw wastewater (filtered to remove large solid matter) was obtained from the influent of the Robert O. Pickard Environmental Centre wastewater plant (Ottawa, Ontario, Canada). Transmission electron microscopy (TEM) grids were purchased from Electron Microscopy Sciences. All acids used were ultrapure reagent grade unless specified. Only double deionized water $\left(\mathrm{ddH}_{2} \mathrm{O}\right)$ was used in this experiment. All other chemicals were purchased from Sigma Aldrich and used as received.

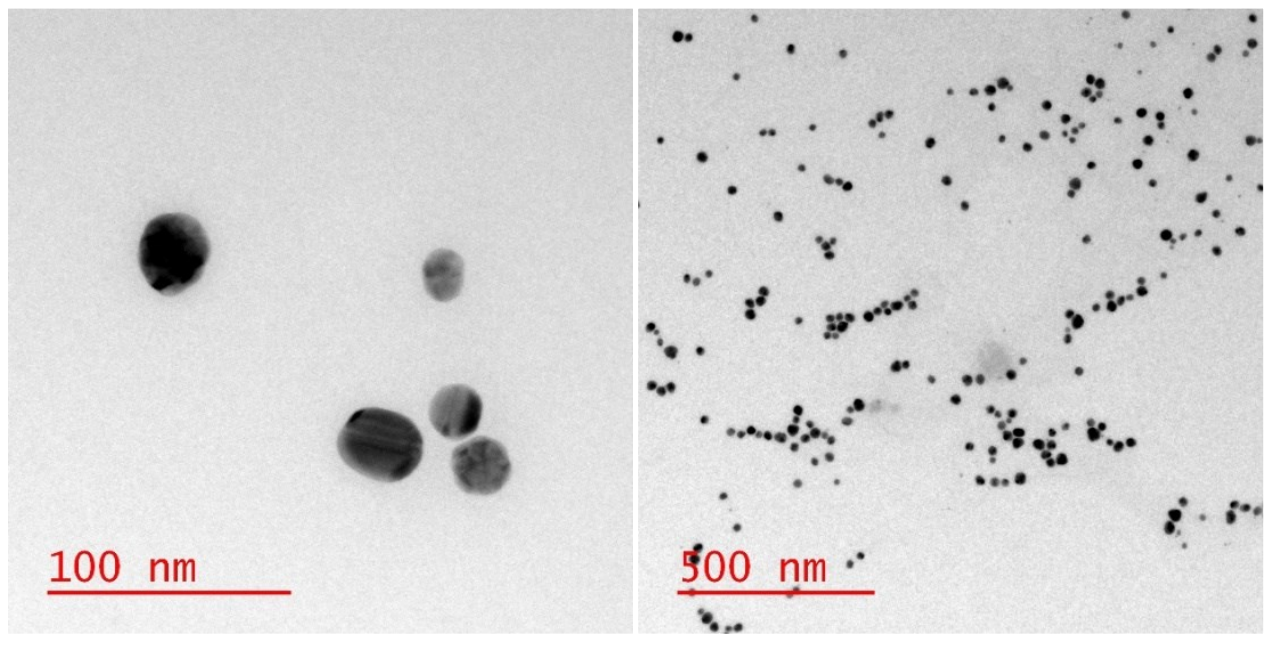

Figure 3-2. TEM images of $25 \mathrm{~nm}$ pristine AgNPs from Nanocomposix in $\mathrm{ddH}_{2} \mathrm{O}$ Left: Close up view showing particles of mostly spherical shape. Right: A full view of the nanoparticle sample, indicating a reasonably monodisperse sample with little to no aggregation. 


\subsubsection{Preparing socks for in-situ impregnation (Kier boiling)}

Kier boiling of the commercial socks was employed to remove the wax finishing and non-cellulosic materials on the cotton fibers ${ }^{46}$. In brief, $14.7 \mathrm{~g}$ of $\mathrm{Na}_{2} \mathrm{CO}_{3}$ and 33.3 grams of $\mathrm{NaOH}$ was added to $1 \mathrm{~L}$ of $\mathrm{ddH}_{2} \mathrm{O}$ in a $2 \mathrm{~L}$ round bottom flask. A pair of white Hanes socks were added to the solution and refluxed for 10 hours. The solution turned yellow when boiling and eventually turned brown, indicating the removal of waxy and non-cellulosic material ${ }^{46}$. The socks were removed from the solution and remained white in color. The socks were then rinsed five times in a $20 \%$ bleach solution and left to air dry.

\subsubsection{In-situ impregnation of Ag nanoparticles into socks (laboratory-prepared socks)}

A kier boiled sock was immersed in a $1 \mathrm{mM} \mathrm{AgNO}_{3}$ solution in a $4 \mathrm{~L}$ beaker at 1:20 (w/v) and then autoclaved for $15 \mathrm{~min}$ at $121^{\circ} \mathrm{C}$ and $15 \mathrm{psi}$. Thereafter, the solution

was left to cool to room temperature ${ }^{47}$. The socks were then rinsed with $\mathrm{ddH}_{2} \mathrm{O}(3 \times 1000$ $\mathrm{mL}$ ) to remove any free NPs and then air-dried. The socks turned from white to brown, indicating the deposition of AgNPs on the socks ${ }^{47}$. 


\subsubsection{Day-to-day wear with sweat, bleach, and detergent}

Each treatment was comprised of a single sock in a 1L beaker with $750 \mathrm{ml}$ of one of three solutions mimicking typical product use. For the sweat solution, a $1.08 \% \mathrm{NaCl}$, $0.12 \%$ lactic acid and $0.13 \%$ urea solution was prepared $(\mathrm{pH} 3)^{34}$. To mimic typical bleaching or laundering conditions, $10 \mathrm{~mL}$ bleach in $750 \mathrm{~mL}$ of water $(\mathrm{pH} \mathrm{11.3)}$ and six $\mathrm{mL}$ of detergent in $750 \mathrm{~mL}$ of water $(\mathrm{pH} 8.6)$, were used, respectively ${ }^{31}$. The solutions were then agitated on an Innova 40 Incubator Shaker at 100 oscillations per minute at 37 ${ }^{\circ} \mathrm{C}$ for 24 hours, to simulate multiple uses and wash cycles as well as the average wash temperature.

\subsubsection{Wastewater exposure}

Raw wastewater was characterized (see supporting information, Table S 3-1) and used the very same day unless indicated. $5 \mu \mathrm{L}$ of the pristine NPs solution $(5 \mathrm{mg} / \mathrm{mL})$ was added to $2 \mathrm{~mL}$ of raw wastewater. The leachate from the bleach, detergent, and sweat solutions was each mixed separately with wastewater at a 1:1 ratio to make up a $2 \mathrm{~mL}$ solution. The mixtures were then vortexed on a Vortex-Genie 2, (Scientific Industries) for 10 minutes, and then left for 24 hours at $21^{\circ} \mathrm{C}$ before the TEM grids were prepared.

A $15 \mathrm{mg} / \mathrm{L}$ aqueous solution of 1,6-hexanedithiol and a $5 \mathrm{mg} / \mathrm{L}$ aqueous solution of ammonium hydroxide were also used as model sulfur-rich and nitrogen-rich solutions, respectively. Pristine NPs and leachate from the bleach, detergent, and sweat solutions 
were directly added to the sulfur or nitrogen solutions, vortexed for $10 \mathrm{~min}$, and left for 24 hours at $21^{\circ} \mathrm{C}$.

The choices for the incubation time and temperatures were guided by the previous work done by our research group ${ }^{48}$.

\subsubsection{Sample preparation for ICP-MS analysis}

\subsubsection{Incineration}

A small pre-weighed piece of the sock $(\sim 100 \mathrm{mg})$ was placed in a $10 \mathrm{ml}$ Pyrex beaker and placed into a TEMCO 1520 benchtop muffle furnace. The furnace was heated to $600{ }^{\circ} \mathrm{C}$ and left for 3 hours or until the sample turned to white ash. The sample was let to cool to room temperature before removing from the furnace ${ }^{34}$. The ash was then dissolved in $100 \mu \mathrm{L} \mathrm{HNO}_{3}$ and diluted $10 \mathrm{X}$ with $\mathrm{ddH}_{2} \mathrm{O}$.

\subsubsection{2 $\underline{\text { Aqua-regia }}$}

A small pre-weighed piece of the sock $(\sim 100 \mathrm{mg})$ was soaked in a 1:3 ratio of $\mathrm{HNO}_{3}$ and $\mathrm{HCl}$ for 1 hour and then heated to $90{ }^{\circ} \mathrm{C}$ for 2 hours ${ }^{49}$. The sample was diluted $10 \mathrm{X}$ with $\mathrm{ddH}_{2} \mathrm{O}$ before analysis.

\subsubsection{3 $\underline{\mathrm{HNO}_{3} / \underline{\mathrm{H}}_{2}} \underline{\mathrm{O}_{2}}$}

A small pre-weighed piece of the sock $(\sim 100 \mathrm{mg})$ was submerged in a 1:1 ratio of $\mathrm{HNO}_{3}$ and $\mathrm{H}_{2} \mathrm{O}$. The solution was heated to $100{ }^{\circ} \mathrm{C}$, and more $\mathrm{HNO}_{3}$ was added slowly until most of the sock dissolved. The solution was allowed to cool, and $30 \% \mathrm{H}_{2} \mathrm{O}_{2}$ was 
added until the bubbling stopped ${ }^{31}$. The sample was diluted $10 \mathrm{X}$ with $\mathrm{ddH}_{2} \mathrm{O}$ before analysis.

\subsubsection{Analysis}

ICP-MS was performed on an Agilent 8800QQQ or an Agilent 7700x ICP-MS (used only to determine the best extraction method). The ICP-MS samples for the socks were prepared by dissolving the ash after incineration in $1 \mathrm{~mL} \mathrm{HNO}_{3}$ and diluting that in

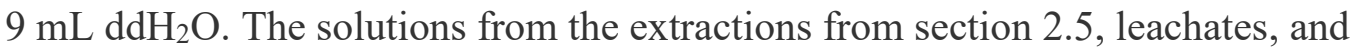
wastewater incubations were used as-is. Appropriate dilutions were made to ensure the Ag concentration was within the calibration curve and the results were normalized to compare the extraction methods.

The TEM images were taken using FEI Tecnai G2 TEM at $200 \mathrm{kV}$. Samples were first prepared the same way as for the ICP-MS. The solutions were then vortexed for two minutes, and thereafter, $6 \mu \mathrm{L}$ of the sample was placed on the grid and left for a minute. Residual liquid was removed using a pipette, and another $6 \mu \mathrm{L}$ of the sample was added. The residual sample was removed for the second time, and the grid was left to air dry for two hours. The CF300-Cu grid was used.

The EDS of the sample was taken through an attachment to the TEM, Aztec EDS, from Oxford X-ray detection systems. The beam was made top focus directly on the nanoparticle. 
The XPS were taken using Kratos analytical model Axis Ultra DLD using $\mathrm{AlK}_{\alpha}$ radiation, charge neutralizer, and a delay-line detector (DLD). The samples used for XPS were all in the liquid form, wherein $10 \mu \mathrm{L}$ of the sample was applied onto a glass coverslip. The sample was left to air dry for two hours. Once dried, another $10 \mu \mathrm{L}$ of the sample was applied to the exact same spot and again left to air dry for two hours. This step was repeated 10 times or more until a visible spot was observed on the glass coverslip.

\subsection{Results and Discussion}

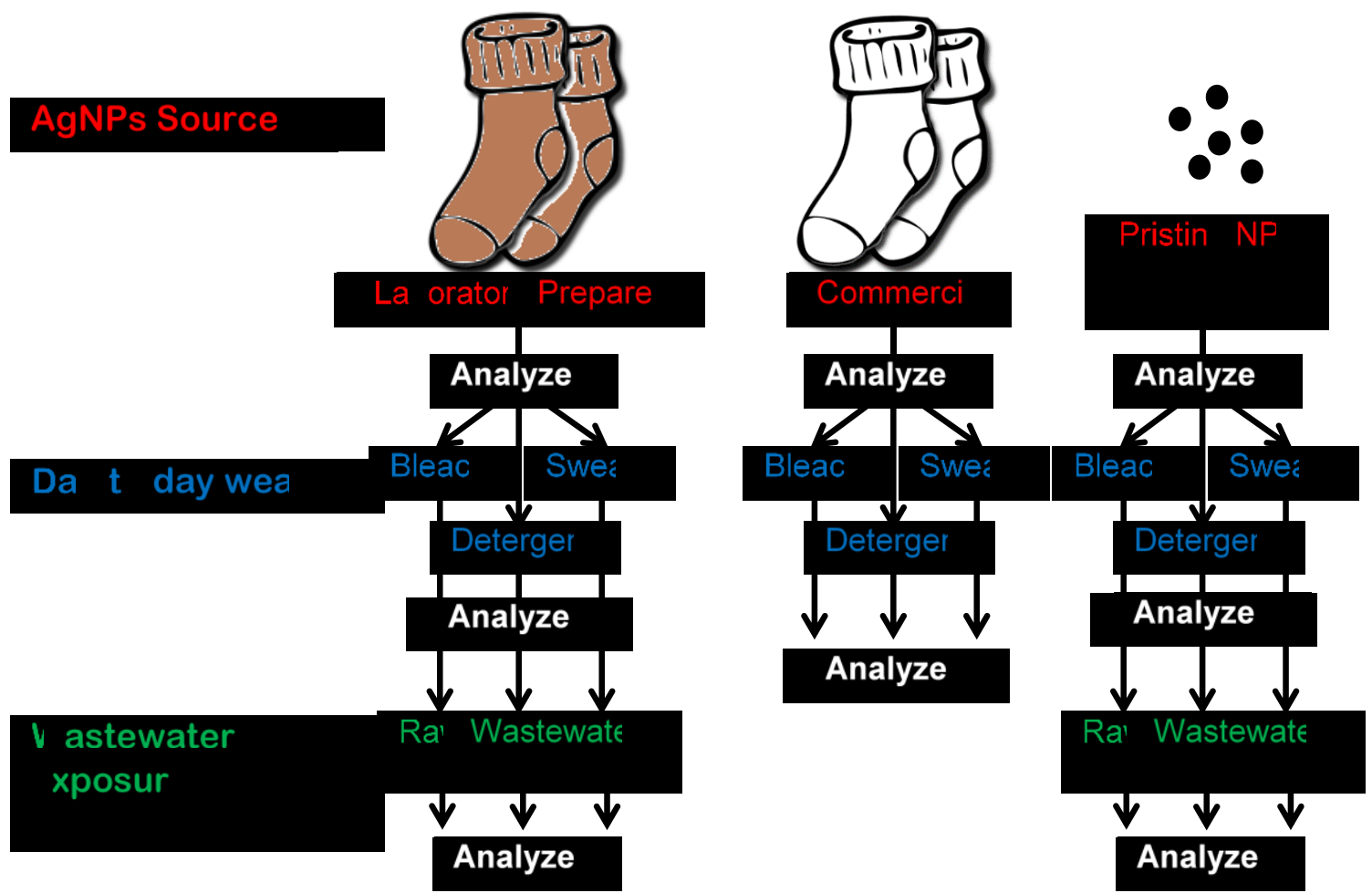

Figure 3-3. Summary of the lifecycle path and analysis of different AgNP sources studied in this paper. All analysis consists of TEM, EDS, and ICP-MS. The commercial socks were only analyzed through ICP-MS. 
The diagram in Figure 3-3 shows the experimental design and path taken in analyzing the transformations of AgNP from three different sources through different use scenarios. The sources of AgNPs studied were the commercial socks, laboratory-prepared AgNP socks (in-situ NP synthesis used in industry ${ }^{50,51}$ ), and pristine AgNPs.

However, in order to analyze the silver content in these socks, either prior to and subsequent to use and wastewater exposure, a suitable method of extraction was required. Three methods (incineration, aqua-regia digestion, and nitric acid/hydrogen peroxide digestion) were selected, and the effectiveness of each technique was evaluated by ICPMS results. The background Ag concentration of the instrument was measured to be 5 ng/L for the ICP-MS. Figure 3-4 shows the effectiveness of each preparation method in extracting Ag from the same commercial sock before day-to-day wear and wastewater exposure. Incinerating the socks yielded the most Ag extracted, confirmed by ANOVA statistical test ( $\mathrm{F}>\mathrm{F}$ crit, $\mathrm{P}=0.000294)$. As a result, incineration was selected as the preferred method of analysis and subsequently used to prepare the remainder of sock samples for analysis. 


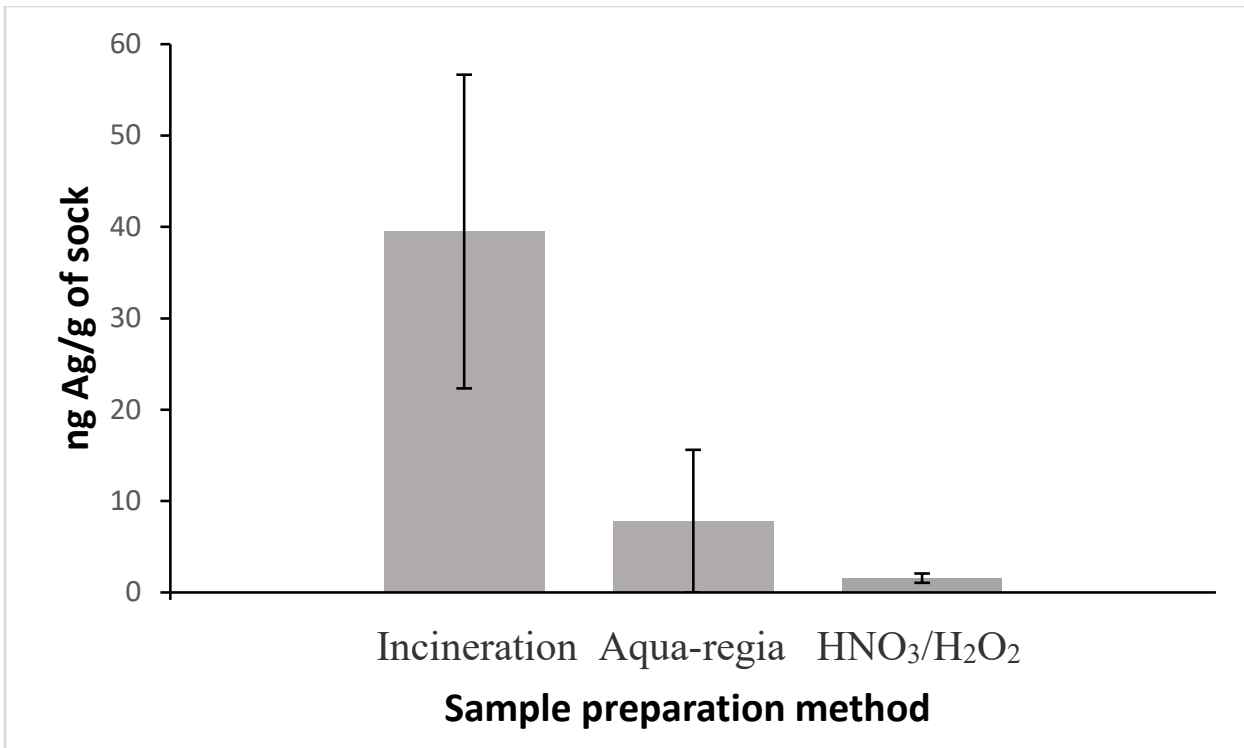

Figure 3-4. Mean ( \pm standard deviation) total Ag, derived from five samples, as measured through ICP-MS, based on different Ag extraction procedures on commercial socks before day-to-day use and wastewater exposure.

Transmission electron microscopy with Energy Dispersive X-ray Spectroscopy (TEM-EDS) was used throughout this study to confirm the presence of AgNPs and to note morphological and compositional changes. However, despite being able to quantify $\mathrm{Ag}$ in these textiles by ICP-MS, the AgNPs could not be found in the commercial socks through TEM and EDS analysis, as shown in Figure 3-5. The NPs found in the TEM images of commercial socks were determined to be a majority of $\mathrm{TiO}_{2} \mathrm{NPs}^{\mathrm{TiO}} \mathrm{Ti}_{2} \mathrm{NPs}$ can also be added as a white pigment within fabrics to mask the brown color of AgNP ${ }^{52}$. The overwhelming presence of $\mathrm{TiO}_{2}$ may have masked the ability to detect $\mathrm{Ag}$ through TEM-EDS. In contrast, the AgNPs could readily be observed in the laboratory-prepared socks with a size distribution of between $5-50 \mathrm{~nm}$ via TEM-EDS. The laboratoryprepared socks were brown as no $\mathrm{TiO}_{2}$ oxide was used to mask the color. 
The Ag-containing socks (commercial and laboratory-prepared socks) were then analyzed for Ag content through ICP-MS, and the results are shown in Table 3-1. The Ag content of the laboratory-prepared sock was about 5000x higher than that of the commercial socks. This low Ag content in the commercial socks was below the detection limit for TEM-EDS. However, the concentration of Ag in the commercial sock is consistent with what has been reported in the literature $2-1360 \mu \mathrm{g}-\mathrm{Ag} / \mathrm{g}$-socks was detected when analyzing 5 different Ag-containing commercial socks ${ }^{53}$. Further analysis of the commercial socks was only done by ICP-MS as the detection of Ag in the commercial socks was not possible via TEM-EDS. 


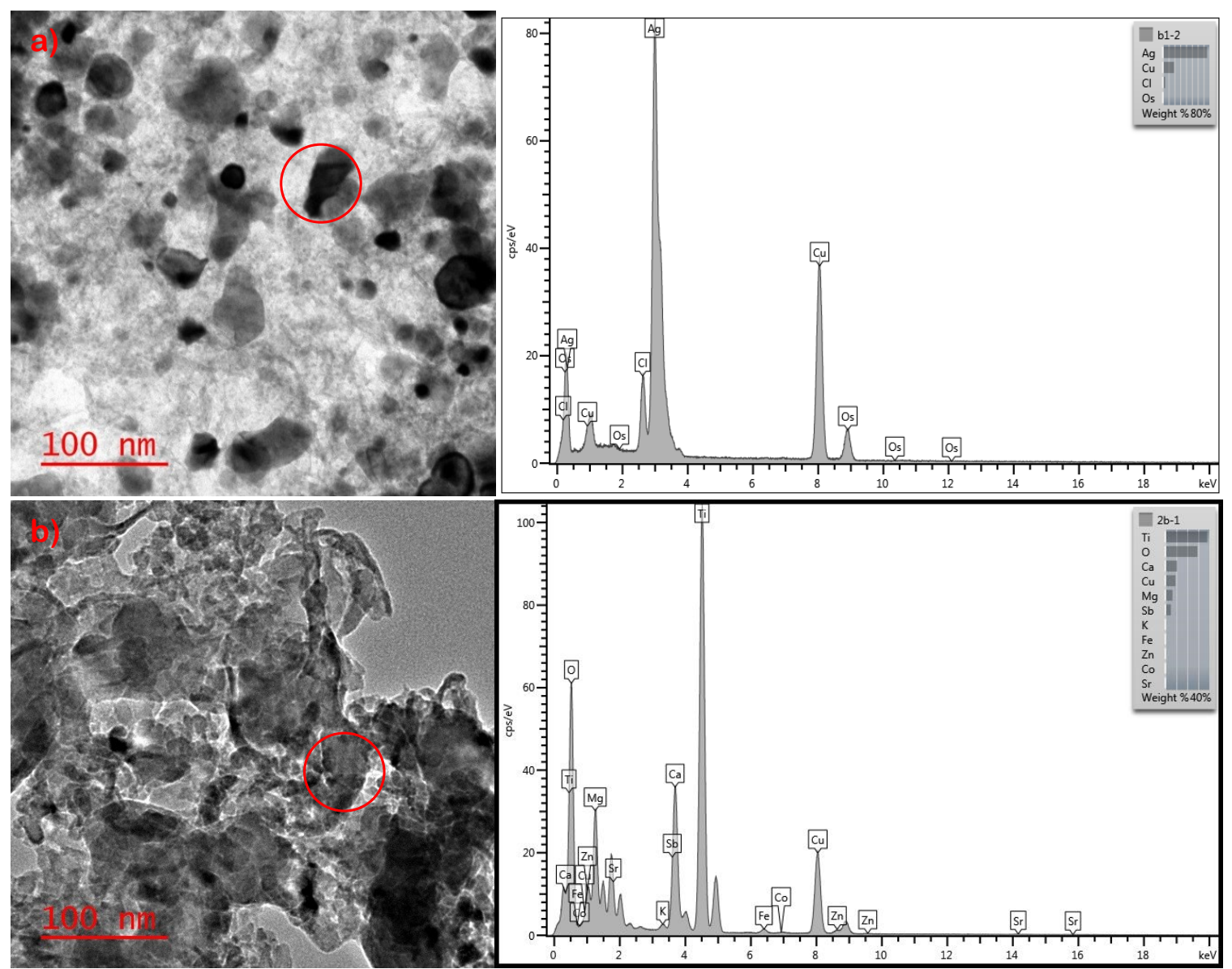

Figure 3-5. TEM and EDS image of a) AgNPs found in laboratory-prepared socks b) Only $\mathrm{TiO}_{2}$ and $\mathrm{ZnO}$ NPs found in commercial socks.

Table 3-1. ICP-MS result of Ag content in AgNP containing socks before any use or wastewater exposure.

\begin{tabular}{|c|c|}
\hline Sample & $\mathrm{mg} / \mathrm{g}$ of sock \\
\hline Commercial socks & $0.0063 \pm 0.0040$ \\
\hline Laboratory-prepared socks & $2.84 \pm 0.47$ \\
\hline
\end{tabular}

Three systems were evaluated through simulated day-to-day use conditions (sweating, washing, and bleaching): the commercial and laboratory-prepared socks, as 
well as free, pristine AgNPs (Figure 3-3). Human sweat varies in its $\mathrm{pH}$ and composition from different individuals. Kulthong et al. (2010) evaluated the effect of artificial sweat of different $\mathrm{pH}$ ranges on both laboratory-prepared and commercial fabrics, and determined that a pH of 6.5 released the most amount of AgNPs ${ }^{34}$. A similar synthetic sweat composition was therefore used in this study ${ }^{34}$. Commercial detergent and bleach were also used in the present study, and concentrations followed that prescribed for use by the commercial products to mimic realistic washing and bleaching conditions. The $\mathrm{pH}$ of bleach, detergent, and sweat solution were 11.3, 8.6 and 2.8 respectively. Another study had shown $\mathrm{pH}$ not to affect the chemical properties of $\mathrm{AgNPs}^{54}$. Exposing AgNPs to solutions of $\mathrm{pH} 2-10$ did not change the zeta potential of the nanoparticles ${ }^{54}$.

The commercial and laboratory-prepared socks were incubated with bleach, detergent, and sweat solutions for 24 hours to mimic day-to-day wear. These solutions were then analyzed using ICP-MS, of which results are shown in Table 3-2. Both the detergent and sweat solution had extracted Ag from the commercial socks, but there was no significant $\mathrm{Ag}$ in the bleach solution. The absence of Ag in the bleach solution could be due to the higher $\mathrm{pH}$ of the solution, limiting the extraction of $\mathrm{Ag}$ from the cotton fibers, or the extracted NPs could have agglomerated and settled, leading to an inhomogeneous sample for ICP-MS. Such large agglomerated Ag particles caused by the bleach solution were observed in TEM images of samples (Figure 3-6 and Figure 3-7). The observed agglomeration of Ag can be due to the oxidative dissolution of AgNPs, followed by the precipitation of $\mathrm{AgCl}^{25}$.

Since the initial concentrations of $\mathrm{Ag}$ were low, TEM images were not taken for any of the solutions from the commercial socks. The laboratory-prepared socks had much 
more silver extracted into solution. However, the ratio of Ag released compared to the total Ag content in the socks was similar between the commercial and laboratoryprepared socks, indicative that the binding strength between the AgNPs and the cotton fibers in the lab-prepared socks resembled the commercial socks. Having similar nanoparticle release ratio makes the laboratory prepared socks a suitable model to yield an accurate representation of the commercial socks for day-to-day use and wastewater exposure.

Table 3-2. Mean concentration ( \pm standard deviation), derived from triplicates, as measured through ICP-MS for Ag extracted from socks into solution after 24-hours of day-to-day use

\begin{tabular}{|c|c|c|c|}
\hline & \multicolumn{3}{|c|}{ Ag concentration } \\
\hline Day-to-day use & Sweat & Bleach & Detergent \\
\hline $\begin{array}{c}\text { Commercial socks (ng/L) } \\
( \pm 5 \text { ng/L) }\end{array}$ & 202 & 5 & 158 \\
\hline $\begin{array}{c}\text { Laboratory-prepared } \\
\text { socks (mg/L) }( \pm 10 \mathrm{mg} / \mathrm{L})\end{array}$ & 441 & 564 & 620 \\
\hline
\end{tabular}

Selected TEM images of the extracts from the laboratory-prepared socks sample exposed to bleach, detergent, and sweat conditions are shown in Figure 3-6 (a, b and c). Apart from the bleach solution, the AgNPs are seen to remain well-dispersed in the extracted solutions. The bleach solutions showed aggregated assemblies, appearing to be nucleated on salt crystals. EDS indicated the association of S and $\mathrm{Cl}$ with AgNPs in the sweat and detergent extracts, while only $\mathrm{Cl}$ could be co-detected with $\mathrm{Ag}$ in the bleach extracts. 
For comparison, pristine NPs were put through the same conditions as the laboratory-prepared socks (Figure 3-6 - d, e, and f). In the sweat solution, the AgNP were mostly well dispersed, and some association on $\mathrm{NaCl}$ salt crystals was observed. The AgNPs extracted from the bleach solution appeared to once again to be aggregated, and $\mathrm{Cl}$ could be co-detected by EDS. Finally, in detergent, small aggregates could be observed with only S co-detected. The pristine AgNPs show a similar transformation as the AgNPs that leach out of the laboratory-prepared socks (Figure 3-6 - a, b, and c).
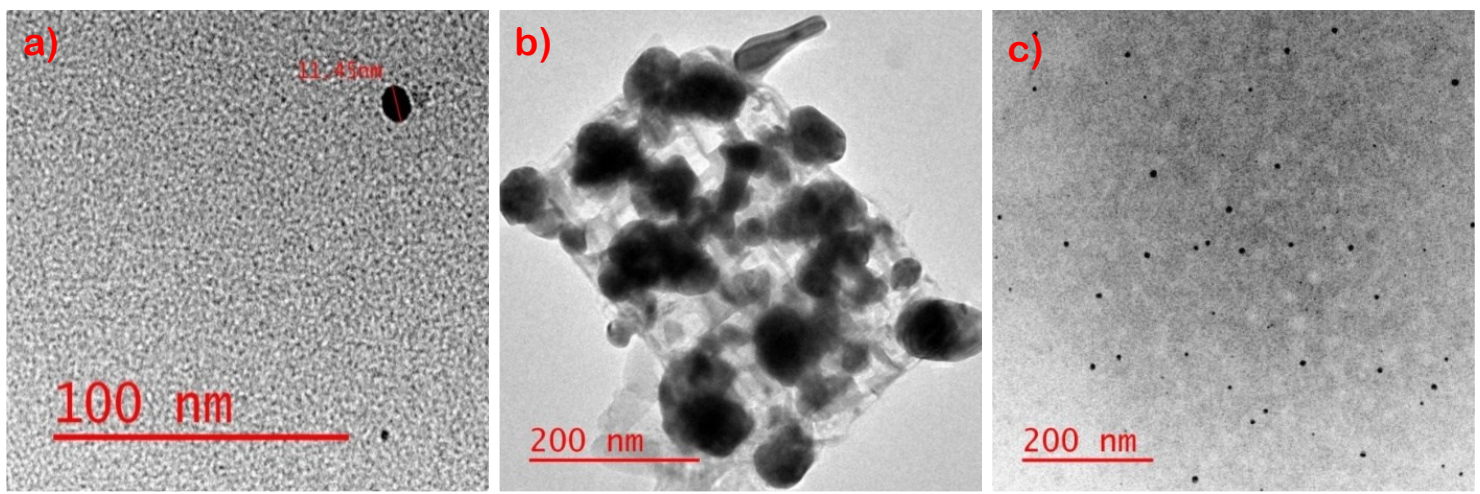

d)
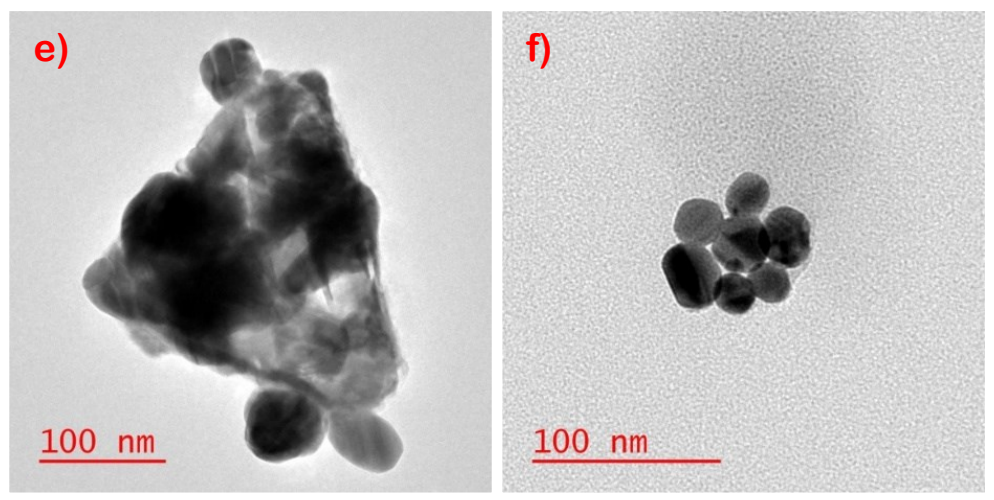

Figure 3-6. Representative TEM images of laboratory-prepared socks weathered with of a) sweat b) bleach, c) detergent for 24-hours and TEM images of pristine NPs weathered with d) sweat e) bleach and f) detergent for 24-hours to mimic day-to-day use. See supporting information Figure S 3-1 and Figure S 3-2 for EDS data

AgNPs extracted out of a textile by sweat, bleach, or detergent could find their way into the wastewater collection system by bathing of the user or washing of the 
textile. It is estimated that wastewater travels within the collection system for a U.S. national average of 3.3 hours up to as long as 15 hours in larger cities before reaching the wastewater treatment facility ${ }^{55}$. During this time, the NPs will be exposed to organic matter, nitrogen, phosphorus, sulfur, metals, and a wide range of chemical compounds and microorganisms. The chemical and microbial reactions, dissolved oxygen concentration, oxidation-reduction potential, ionic strength, and $\mathrm{pH}$ of wastewater will change as a function of residence time in the sewer, which can physically and chemically transform the AgNPs. The effect of incubation in raw wastewater was compared for the AgNPs that had been extracted through the various use-scenarios, as well as pristine NPs, to observe effects on morphology or composition.

The solutions obtained from bleach, detergent, and sweat extraction of laboratoryprepared socks were added 1:1 to wastewater to observe the effects on the AgNPs. (Figure 3-7). With the bleach solution, the AgNPs agglomerated as their average diameter increased from 5-50 $\mathrm{nm}$ to $>100 \mathrm{~nm}$. In contrast, the particle sizes detected from the detergent and sweat solutions post-wastewater incubation did not show an increase of particle size. However, halos with high $\mathrm{S}$ and $\mathrm{Cl}$ content could be observed around the dispersed particles suggesting a respective association with Ag and potentially dissolution and re-precipitation.

The solutions obtained from bleach, detergent, and sweat of pristine NPs were also examined after a 24-hour incubation in wastewater (Figure 3-7). Larger aggregates and agglomerates with low levels of $\mathrm{Cl}$ content could once again be observed in the bleach sample. NPs in the sweat solution appeared to be similar to those in the bleach 
solution. The detergent samples appeared more dispersed and significant association with $\mathrm{Cl}$ and $\mathrm{S}$ was observed.
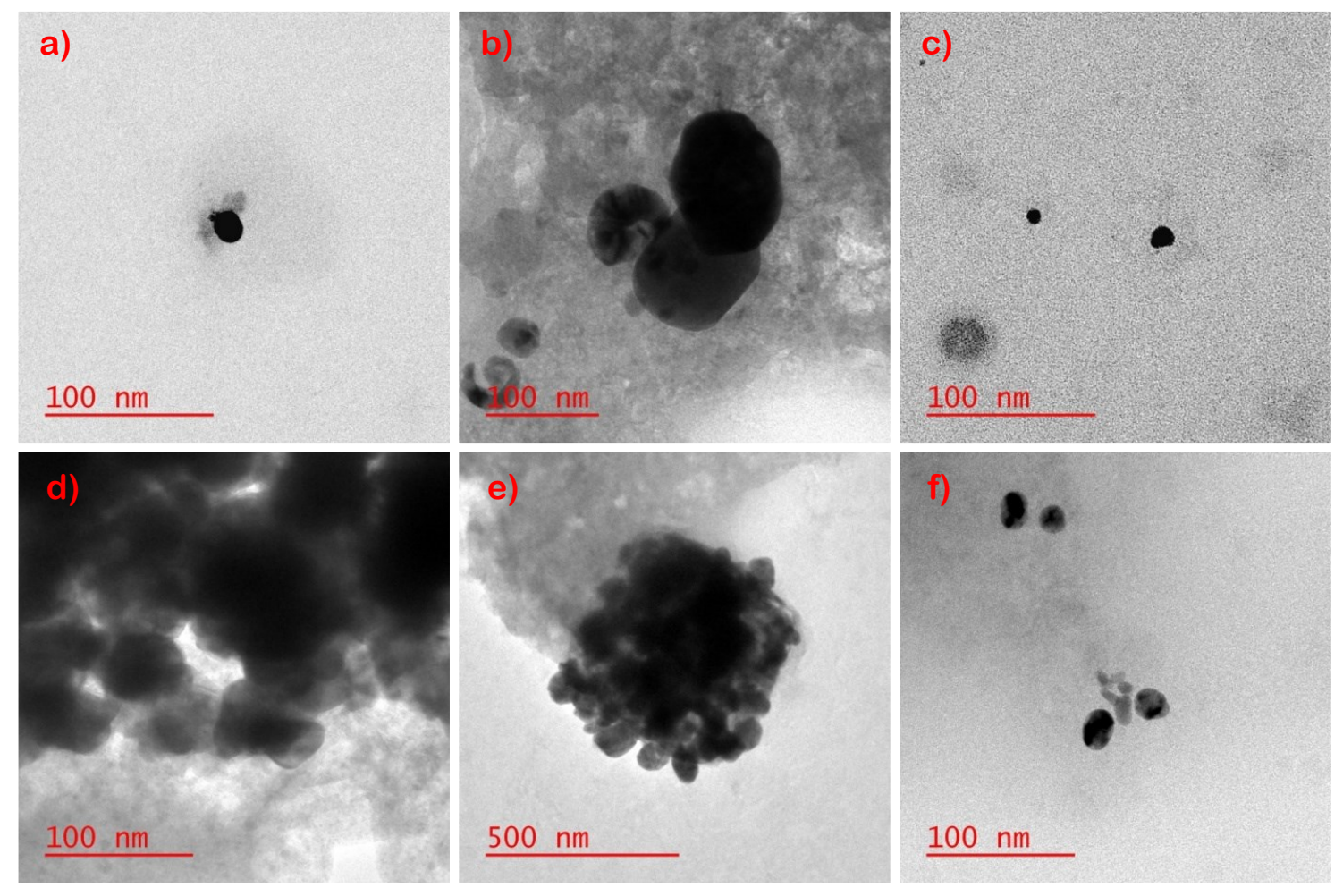

Figure 3-7. Representative TEM images of a) sweat b) bleach, c) detergent solution from laboratory-prepared socks and TEM images of d) sweat e) bleach f) detergent solution from pristine NPs after 24-hour incubation with wastewater. See supporting information Figure S 3-3 and Figure S 3-4 for EDS data.

In order to have a better understanding of the effect of wastewater composition on AgNP morphology and composition, pristine AgNPs that had not undergone any pretreatment were introduced to the wastewater, as well as sulfur-rich and nitrogen-rich solutions, and 1:1 wastewater/ sulfur solution and wastewater/nitrogen solutions (Figure S 3-5). Pristine AgNPs incubated in wastewater became aggregated, and sulfur-rich halos were observed around the particles. The introduction of the AgNPs to the model sulfur- 
rich solutions showed similar morphology effects to that observed with wastewater, while exposure to our nitrogen-rich solution did not lead to aggregation or show formations of halos. The detection of nitrogen could be because of ammonia forming a stable ligand with $\mathrm{Ag}, \mathrm{Ag}\left(\mathrm{NH}_{3}\right)_{2}^{+}$which is stabilized by the $\mathrm{OH}^{-}$ions in solution ${ }^{56}$. Similar results were observed by adding pristine NPs to mixtures of wastewater + sulfur-rich solution and wastewater + nitrogen-rich solution. The effects of different wastewater $\mathrm{pH}(4.0,6.0$ \& 8.0) were also tested (Figure $\mathbf{S}$ 3-6). The change in $\mathrm{pH}$ did not affect the physical form or aggregation state of AgNPs. Pristine nanoparticles had been shown to start aggregating when the $\mathrm{pH}$ drops below $\mathrm{pH} 3.0^{54}$.

To further analyze these chemical changes, samples of pristine AgNPs incubated in wastewater were analyzed using XPS. In Figure 3-8, a shift in binding energy was observed for Ag when the nanoparticles were incubated with wastewater. The shift to higher binding energy is caused by the oxidation of the $\mathrm{Ag}$ atom ${ }^{57}$. The shift of the binding energy further supports the association of $\mathrm{S}$ and $\mathrm{Cl}$ with $\mathrm{AgNPs}$ observed in EDS are due to the AgNPs going through a chemical transformation ${ }^{58}$. 


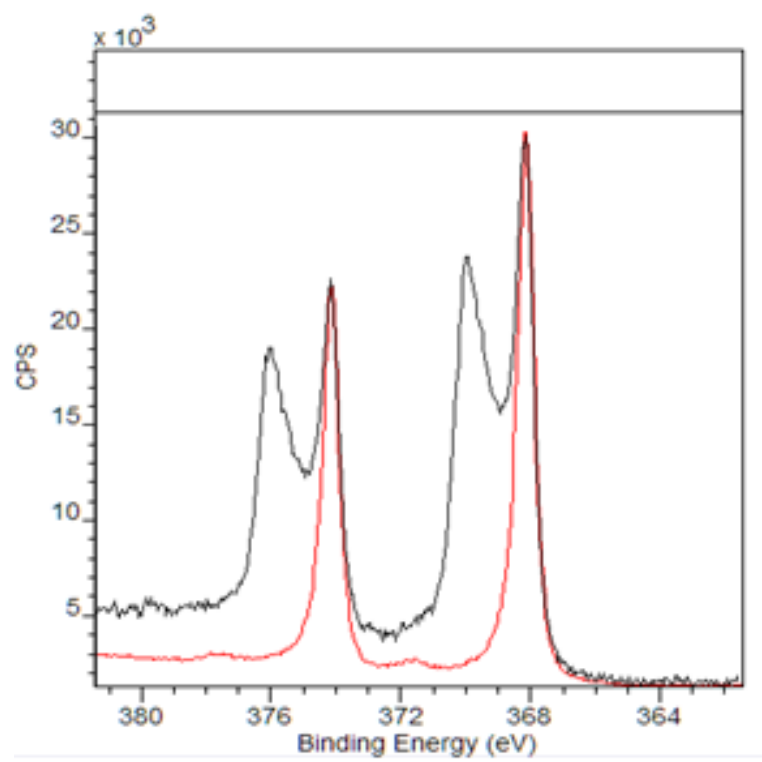

Figure 3-8. XPS spectra showing the binding energy of Ag of pristine AgNPs (red) and those incubated in wastewater for 24-hours (black) obtained through high-resolution XPS.

Table 3-3Error! Reference source not found. below shows the summary based on all the TEM images and EDS data from the samples. The nanoparticles were observed to aggregate, agglomerate, and associate with chlorine and sulfur when exposed to wastewater, regardless of origin and previous weathering. Images shown in this paper were selected as being the best representation of the AgNPs on the $100 \mathrm{~nm}$ scale. Some agglomerations were observed to reach the microscale, which supports the recent reports where pristine AgNP exposed to simulated detergent and bleach wash cycles form large agglomerates ${ }^{33,59}$. The elemental association with $\mathrm{Cl}$ and $\mathrm{S}$ observed with EDS is likely because of the formation of $\mathrm{AgCl}$ and $\mathrm{Ag}_{2} \mathrm{~S}$. A similar interpretation of the EDS data for AgNPs in simulated wash cycles had been reported in a recent study ${ }^{35}$.

However, not all the nanoparticles imaged and analyzed appeared to have oxidized to $\mathrm{AgCl}$ and $\mathrm{Ag}_{2} \mathrm{~S}$. According to a previous study that exposed commercial 
AgNP-laden fabric to the bleach and detergent, only $50 \%$ of the AgNPs were converted to $\mathrm{AgCl}^{60}$. Since X-ray Absorption Near Edge Spectroscopy (XANES) was used for elemental analysis in that study, only the surface of the nanoparticles was analyzed for the presence of $\mathrm{AgCl}$. The percent $\mathrm{AgCl}$ would potentially be lower if the entire core of the nanoparticle was analyzed ${ }^{61}$. $\mathrm{AgCl}$ and $\mathrm{Ag}_{2} \mathrm{~S}$ are stable forms of ionic silver with $\mathrm{Ag}_{2} \mathrm{~S}$ being the more stable form in the environment ${ }^{62}$. Recent research had shown that despite the lack of short term toxicity of $\mathrm{Ag}_{2} \mathrm{~S}$, long term toxic effects were observed on soil microflora ${ }^{63}$. AgNPs had been shown to form passivation layers of $\mathrm{Ag}_{2} \mathrm{~S}$ on the surface of the nanoparticles. The passivation slows down the dissolution of the nanoparticle but does not completely stop it ${ }^{64}$. The dissolution as $\mathrm{Ag}^{+}$ions continues to impart toxic effects on microbes ${ }^{64}$. 
Table 3-3. Summary of the type of morphological changes and chemical associations of AgNPs when pristine or embedded into the fabric and applied to various use and exposure conditions.

\begin{tabular}{|c|c|c|c|c|}
\hline \multirow[t]{2}{*}{ Samples } & \multirow[t]{2}{*}{ Conditions } & \multicolumn{3}{|c|}{ Changes } \\
\hline & & Agglomeration $^{23}$ & Aggregation $^{23}$ & $\begin{array}{l}\text { Elemental } \\
\text { Association }\end{array}$ \\
\hline \multirow{6}{*}{$\begin{array}{l}\text { Laboratory- } \\
\text { prepared } \\
\text { Socks }\end{array}$} & Sweat & No & No & $\mathrm{Cl}, \mathrm{S}$ \\
\hline & Bleach & Yes & Yes & $\mathrm{Cl}$ \\
\hline & Detergent & No & No & $\mathrm{Cl}, \mathrm{S}$ \\
\hline & $\begin{array}{c}\text { Sweat }+ \\
\text { Wastewater }\end{array}$ & Yes & Yes & $\mathrm{Cl}, \mathrm{S}$ \\
\hline & $\begin{array}{c}\text { Bleach }+ \\
\text { Wastewater }\end{array}$ & Yes & Yes & $\mathrm{Cl}, \mathrm{S}$ \\
\hline & $\begin{array}{l}\text { Detergent + } \\
\text { Wastewater }\end{array}$ & Yes & Yes & $\mathrm{Cl}, \mathrm{S}$ \\
\hline \multirow{6}{*}{$\begin{array}{c}\text { Pristine } \\
\text { Nanoparticles }\end{array}$} & Sweat & No & Yes & $\mathrm{Cl}$ \\
\hline & Bleach & Yes & Yes & $\mathrm{Cl}$ \\
\hline & Detergent & No & Yes & $\mathrm{Cl}, \mathrm{S}$ \\
\hline & $\begin{array}{c}\text { Sweat }+ \\
\text { Wastewater }\end{array}$ & Yes & Yes & $\mathrm{Cl}, \mathrm{S}$ \\
\hline & $\begin{array}{c}\text { Bleach + } \\
\text { Wastewater } \\
\end{array}$ & Yes & Yes & $\mathrm{Cl}, \mathrm{S}$ \\
\hline & $\begin{array}{l}\text { Detergent }+ \\
\text { Wastewater }\end{array}$ & Yes & Yes & $\mathrm{Cl}, \mathrm{S}$ \\
\hline
\end{tabular}

Despite the qualitative nature of this study, some important conclusions can be drawn about the transformations of AgNPs during these simulated day-to-day use and wastewater exposure processes. While AgNPs do not retain their pristine form from product incorporation through use scenarios, to travel in the wastewater collection stream, they are also not fully dissolved throughout these steps. Given that others have detected AgNPs in the wastewater influent and effluent as well as in sewage sludge, our 
use of these simulated scenarios represents the real-world scenarios of the nanoparticle

life-cycle ${ }^{45,65}$. Furthermore, despite evidence for transformations throughout the use and exposure steps, the most dramatic changes to the nanoparticle morphology and composition appear to have occurred in our final step mimicking the time spent in the wastewater collection stream. The presence of both biotic and abiotic factors in wastewater causes the most pronounced transformation in the nanoparticles ${ }^{66}$. Numerous ligands, thiols and dissolved organic matter and inorganic ligands such as chlorides in wastewater will modify the speciation and cause the dissolution of AgNPs ${ }^{65}$. Moreover, the interaction and uptake of AgNPs by the microbes in wastewater will further transform the nanoparticles due to the various cellular processes ${ }^{67}$. As a result, using a 24-hour wastewater incubation of pristine nanoparticles alone may be sufficient to prepare a more relevant model "weathered nanoparticle" sample that can be used for environmental studies seeking to inform future decision making. In future studies, further transformations on the weathered AgNPs through scenarios that mimic wastewater and biosolid treatment processes will be explored.

\subsection{Acknowledgments}

MCD and BO acknowledge Environment and Climate Change Canada for a Grants and Contributions Agreement to support this research. This work would not be possible without the technical help of Jianqun Wang, the TEM and SEM facility manager in obtaining TEM images and troubleshooting the instrument. We would like to thank Nimal DeSilva for his assistance in the ICP-MS analysis of our sample. 


\subsection{References}

1. Zhang, Z. \& Lin, P.-C. Noble metal nanoparticles: synthesis, and biomedical implementations. Emerg. Appl. Nanoparticles Archit. Nanostructures 177-233 (2018). doi:10.1016/B978-0-323-51254-1.00007-5

2. Cassano, D., David, J., Luin, S. \& Voliani, V. Passion fruit-like nanoarchitectures: a general synthesis route. Sci. Rep. 7, 43795 (2017).

3. The Project on Emerging Nanotechnologies. (2013). Available at: http://www.nanotechproject.org/cpi/about/analysis/. (Accessed: 1st April 2015)

4. Vance, M. E. et al. Nanotechnology in the real world: Redeveloping the nanomaterial consumer products inventory. Beilstein J. Nanotechnol. 6, 17691780 (2015).

5. Walser, T., Demou, E., Lang, D. J. \& Hellweg, S. Prospective environmental life cycle assessment of nanosilver T-shirts. Environ. Sci. Technol. 45, 4570-8 (2011).

6. Colman, B. P. et al. Low Concentrations of Silver Nanoparticles in Biosolids Cause Adverse Ecosystem Responses under Realistic Field Scenario. PLoS One 8, (2013).

7. Panyala, N. R., Peña-Méndez, E. M. \& Havel, J. Silver or silver nanoparticles: a hazardous threat to the environment and human health? J. Appl. Biomed. 6, 117129 (2008).

8. Miseljic, M. \& Olsen, S. I. Life-cycle assessment of engineered nanomaterials: a literature review of assessment status. J. Nanoparticle Res. 16, 2427 (2014).

9. Diez-Ortiz, M. et al. Short-term soil bioassays may not reveal the full toxicity potential for nanomaterials; bioavailability and toxicity of silver ions (AgNO3) and silver nanoparticles to earthworm Eisenia fetida in long-term aged soils. Environ. Pollut. 203, 191-198 (2015).

10. Fernández, M. D., Alonso-Blázquez, M. N., García-Gómez, C. \& Babin, M. Evaluation of zinc oxide nanoparticle toxicity in sludge products applied to agricultural soil using multispecies soil systems. Sci. Total Environ. 497-498, 688-96 (2014).

11. Fabrega, J., Fawcett, S. R., Renshaw, J. C. \& Lead, J. R. Silver nanoparticle impact on bacterial growth: effect of $\mathrm{pH}$, concentration, and organic matter. Environ. Sci. Technol. 43, 7285-90 (2009).

12. Gao, J., Lin, L., Wei, A. \& Sepúlveda, M. S. Protein Corona Analysis of Silver Nanoparticles Exposed to Fish Plasma. Environ. Sci. Technol. Lett. 4, 174-179 (2017). 
13. Shukla, S., Seal, S. \& Mishra, S. R. Synthesis and Characterization of Silver Sulfide Nanoparticles Containing Sol-Gel Derived HPC-Silica Film for IonSelective Electrode Application. J. Sol-Gel Sci. Technol. 23, 151-164 (2002).

14. Kim, D. et al. Selective Killing of Pathogenic Bacteria by Antimicrobial Silver Nanoparticle-Cell Wall Binding Domain Conjugates. ACS Appl. Mater. Interfaces 10, 13317-13324 (2018).

15. Navarro, E. et al. Toxicity of silver nanoparticles to Chlamydomonas reinhardtii. Environ. Sci. Technol. 42, 8959-8964 (2008).

16. Fabrega, J., Luoma, S. N., Tyler, C. R., Galloway, T. S. \& Lead, J. R. Silver nanoparticles: Behaviour and effects in the aquatic environment. Environ. Int. 37, 517-531 (2011).

17. Laban, G., Nies, L. F., Turco, R. F., Bickham, J. W. \& Sepúlveda, M. S. The effects of silver nanoparticles on fathead minnow (Pimephales promelas) embryos. Ecotoxicology 19, 185-195 (2010).

18. Dhas, S. P., Shiny, P. J., Khan, S., Mukherjee, A. \& Chandrasekaran, N. Toxic behavior of silver and zinc oxide nanoparticles on environmental microorganisms. J. Basic Microbiol. 916-927 (2013). doi:10.1002/jobm.201200316

19. Prabhu, S. \& Poulose, E. K. Silver nanoparticles: mechanism of antimicrobial action, synthesis, medical applications, and toxicity effects. International Nano Letters 2, 32 (2012).

20. Marambio-Jones, C. \& Hoek, E. M. V. A review of the antibacterial effects of silver nanomaterials and potential implications for human health and the environment. J. Nanoparticle Res. 12, 1531-1551 (2010).

21. El-Shishtawy, R. M., Asiri, A. M., Abdelwahed, N. a M. \& Al-Otaibi, M. M. In situ production of silver nanoparticle on cotton fabric and its antimicrobial evaluation. Cellulose 18, 75-82 (2011).

22. Servin, A. D. \& White, J. C. Nanotechnology in agriculture: Next steps for understanding engineered nanoparticle exposure and risk. NanoImpact 1, 9-12 (2016).

23. Nichols, G. et al. A review of the terms agglomerate and aggregate with a recommendation for nomenclature used in powder and particle characterization. $J$. Pharm. Sci. 91, 2103-2109 (2002).

24. Mitrano, D. M. et al. Presence of nanoparticles in wash water from conventional silver and nano-silver textiles. ACS Nano 8, 7208-19 (2014).

25. Garg, S., Rong, H., Miller, C. J. \& Waite, T. D. Oxidative Dissolution of Silver Nanoparticles by Chlorine: Implications to Silver Nanoparticle Fate and Toxicity. 
Environ. Sci. Technol. 50, 3890-6 (2016).

26. Meier, C. et al. Transformation of Silver Nanoparticles in Sewage Sludge during Incineration. Environ. Sci. Technol. 50, 3503-10 (2016).

27. Dale, A. L., Lowry, G. V \& Casman, E. A. Modeling nanosilver transformations in freshwater sediments. Environ. Sci. Technol. 47, 12920-8 (2013).

28. Chambers, B. A. et al. Effects of chloride and ionic strength on physical morphology, dissolution, and bacterial toxicity of silver nanoparticles. Environ. Sci. Technol. 48, 761-9 (2014).

29. Liu, J., Wang, Z., Liu, F. D., Kane, A. B. \& Hurt, R. H. Chemical transformations of nanosilver in biological environments. ACS Nano 6, 9887-99 (2012).

30. Nowack, B. et al. Potential scenarios for nanomaterial release and subsequent alteration in the environment. Environ. Toxicol. Chem. 31, 50-9 (2012).

31. Benn, T. M. \& Westerhoff, P. Nanoparticle silver released into water from commercially available sock fabrics. Environ. Sci. Technol. 42, 4133-9 (2008).

32. Mitrano, D. M., Lombi, E., Dasilva, Y. A. R. \& Nowack, B. Unraveling the Complexity in the Aging of Nanoenhanced Textiles: A Comprehensive Sequential Study on the Effects of Sunlight and Washing on Silver Nanoparticles. Environ. Sci. Technol. 50, 5790-5799 (2016).

33. Hedberg, J. et al. Sequential studies of silver released from silver nanoparticles in aqueous media simulating sweat, laundry detergent solutions and surface water. Environ. Sci. Technol. 48, 7314-7322 (2014).

34. Kulthong, K., Srisung, S., Boonpavanitchakul, K., Kangwansupamonkon, W. \& Maniratanachote, R. Determination of silver nanoparticle release from antibacterial fabrics into artificial sweat. Part. Fibre Toxicol. 7, 8 (2010).

35. Lorenz, C. et al. Characterization of silver release from commercially available functional (nano)textiles. Chemosphere 89, 817-24 (2012).

36. Brar, S. K., Verma, M., Tyagi, R. D. \& Surampalli, R. Y. Engineered nanoparticles in wastewater and wastewater sludge - Evidence and impacts. Waste Manag. 30, 504-520 (2010).

37. Sheng, Z. \& Liu, Y. Effects of silver nanoparticles on wastewater biofilms. Water Res. 45, 6039-6050 (2011).

38. Hou, L. et al. Removal of silver nanoparticles in simulated wastewater treatment processes and its impact on COD and NH 4 reduction. Chemosphere 87, 248-252 (2012). 
39. García, A. et al. Effect of cerium dioxide, titanium dioxide, silver, and gold nanoparticles on the activity of microbial communities intended in wastewater treatment. J. Hazard. Mater. 199-200, 64-72 (2012).

40. Kaegi, R. et al. Release of silver nanoparticles from outdoor facades. Environ. Pollut. 158, 2900-2905 (2010).

41. Kaiser, J. P., Roesslein, M., Diener, L. \& Wick, P. Human health risk of ingested nanoparticles that are added as multifunctional agents to paints: An in vitro study. PLoS One 8, 1-11 (2013).

42. Kaegi, R. et al. Fate and transformation of silver nanoparticles in urban wastewater systems. Water Res. 47, 3866-3877 (2013).

43. Martin, L. \& Kelso, G. Use of biosolids in agriculture. NSW DPI Primefacts (2009).

44. Kaegi, R. et al. Behavior of metallic silver nanoparticles in a pilot wastewater treatment plant. Environ. Sci. Technol. 45, 3902-3908 (2011).

45. Kim, B., Park, C.-S. S., Murayama, M. \& Hochella, M. F. Discovery and characterization of silver sulfide nanoparticles in final sewage sludge products. Environ. Sci. Technol. 44, 7509-7514 (2010).

46. Karande, V. S., Bharimalla, A. K., Vigneshwaran, N., Kadam, P. G. \& Mhaske, S. T. Cotton linter nano-fibers as the potential reinforcing agent for guar gum. Iran. Polym. J. 23, 869-879 (2014).

47. Vigneshwaran, N., Kathe, A. ., Varadarajan, P. ., Nachane, R. . \& Balasubramanya, R. . Functional Finishing of Cotton Fabrics Using Silver Nanoparticles. J. Nanosci. Nanotechnol. 7, 1893-1897 (2007).

48. Douad, M., Unpublished.

49. Mwilu, S. K. et al. Separation and measurement of silver nanoparticles and silver ions using magnetic particles. Sci. Total Environ. 472, 316-23 (2014).

50. LLC, N.-T. Nanoparticle-based permanent treatments for textiles. US6607994 B2 (2002).

51. University, B.-I. Sonochemical coating of textiles with metal oxide nanoparticles for antimicrobial fabrics. EP2294260 A1 (2010). doi:PCT/IL2009/000645

52. Eastern Research Group. State of the Science Literature Review : Nano Titanium Dioxide Environmental Matters. Epa (2010).

53. Benn, T. M. \& Westerhoff, P. Nanoparticle Silver Released into Water from Commercially Available Sock Fabrics. Environ. Sci. Technol. 42, 4133-4139 
(2008).

54. Badawy, A. M. El et al. Impact of Environmental Conditions (pH, Ionic Strength, and Electrolyte Type) on the Surface Charge and Aggregation of Silver Nanoparticles Suspensions. Environ. Sci. Technol. 44, 1260-1266 (2010).

55. Kapo, K. E., Paschka, M., Vamshi, R., Sebasky, M. \& McDonough, K. Estimation of U.S. sewer residence time distributions for national-scale risk assessment of down-the-drain chemicals. Sci. Total Environ. 603-604, 445-452 (2017).

56. Fox, B. S., Beyer, M. K. \& Bondybey, V. E. Coordination chemistry of silver cations. J. Am. Chem. Soc. 124, 13613-13623 (2002).

57. Hu, P., Hou, D., Huang, Y., Hu, X. \& Chen, C. Biomaterial-assisted synthesis of $\mathrm{AgCl} @ \mathrm{Ag}$ concave cubes with efficient visible-light-driven photocatalytic activity. CrystEngComm 16, 649-653 (2013).

58. Kaushik, V. K. XPS core level spectra and Auger parameters for some silver compounds. J. Electron Spectros. Relat. Phenomena 56, 273-277 (1991).

59. Geranio, L., Heuberger, M. \& Nowack, B. The behavior of silver nanotextiles during washing. Environ. Sci. Technol. 43, 8113-8118 (2009).

60. Impellitteri, C. A., Tolaymat, T. M. \& Scheckel, K. G. The Speciation of Silver Nanoparticles in Antimicrobial Fabric Before and After Exposure to a Hypochlorite/Detergent Solution. J. Environ. Qual. 38, 1528-1530 (2009).

61. Hemraj-Benny, T. et al. Near-Edge X-ray Absorption Fine Structure Spectroscopy as a Tool for Investigating Nanomaterials. Small 2, 26-35 (2006).

62. Yin, Y. et al. Photo- and thermo-chemical transformation of $\mathrm{AgCl}$ and $\mathrm{Ag} 2 \mathrm{~S}$ in environmental matrices and its implication. Environ. Pollut. 220, 955-962 (2017).

63. Kraas, M. et al. Long-term effects of sulfidized silver nanoparticles in sewage sludge on soil microflora. Environ. Toxicol. Chem. 36, 3305-3313 (2017).

64. Le Ouay, B. \& Stellacci, F. Antibacterial activity of silver nanoparticles: A surface science insight. Nano Today 10, 339-354 (2015).

65. Cervantes-Avilés, P., Huang, Y. \& Keller, A. A. Incidence and persistence of silver nanoparticles throughout the wastewater treatment process. Water Res. 156, 188-198 (2019).

66. Azimzada, A., Tufenkji, N. \& Wilkinson, K. J. Transformations of silver nanoparticles in wastewater effluents: links to Ag bioavailability. Environ. Sci. Nano 4, 1339-1349 (2017).

67. Tripathi, D. K. et al. Uptake, Accumulation and Toxicity of Silver Nanoparticle in 
Autotrophic Plants, and Heterotrophic Microbes: A Concentric Review. Front. Microbiol. 08, 7 (2017). 


\subsection{Supporting Information}

Table S 3-1: The test results for raw wastewater composition. Sample 1 of raw wastewater was used for the pristine NPs. Sample 2 of raw wastewater was used for the solutions from day-to-day wear of laboratory-prepared socks and pristine NPs.

\begin{tabular}{|c|c|c|c|}
\hline Test & Units & Sample 1 & Sample 2 \\
\hline $\begin{array}{c}\text { Chemical Oxygen Demand, } \\
\text { COD }\end{array}$ & $\mathrm{mg} / \mathrm{L}$ & 434 & 495 \\
\hline Total Nitrogen, N & $\mathrm{mg} / \mathrm{L} \mathrm{N}$ & 47 & 51 \\
\hline Total Phosphorus, $\mathrm{mg} / \mathrm{L} \mathrm{PO}_{4}{ }^{3-}$ & 28 & 42 \\
\hline Total Sulfate, S & $\mathrm{mg} / \mathrm{L} \mathrm{SO}_{4}-$ & 60 & 87 \\
\hline Ammonia, $\mathrm{NH}_{3}-\mathrm{N}$ & $\mathrm{mg} / \mathrm{L} \mathrm{NH}_{3}-\mathrm{N}$ & 30.9 & 33 \\
\hline Nitrate, NO $3-\mathrm{N}$ & $\mathrm{mg} / \mathrm{L} \mathrm{NO}_{3}{ }^{-}-\mathrm{N}$ & 0.9 & $1 *$ \\
\hline Turbidity & $\mathrm{NTU}$ & 35 & 84 \\
\hline Absorbance $@ 254 \mathrm{~nm}$ & - & 1.0489 & 1.5692 \\
\hline Total Solids & $\mathrm{mg} / \mathrm{L}$ & 1655.6 & 1072.5 \\
\hline Total Volatile Solids & $\mathrm{mg} / \mathrm{L}$ & 1189 & 392 \\
\hline
\end{tabular}

*Test performed the following day 

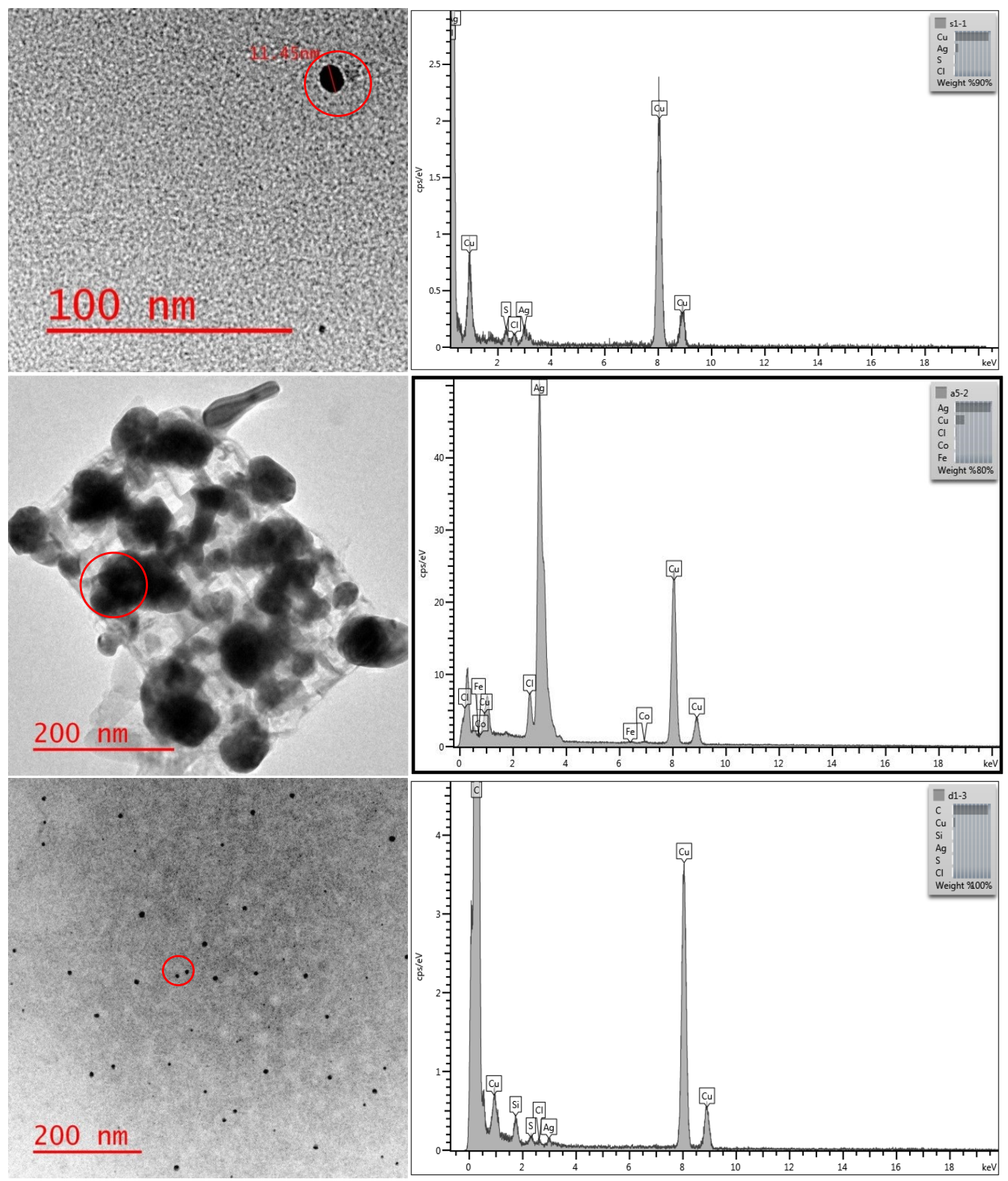

Figure S 3-1: TEM image and EDS data of extracted samples from laboratory-prepared socks exposed to a) sweat b) bleach, c) detergent. Circles indicate areas probed for EDS. 


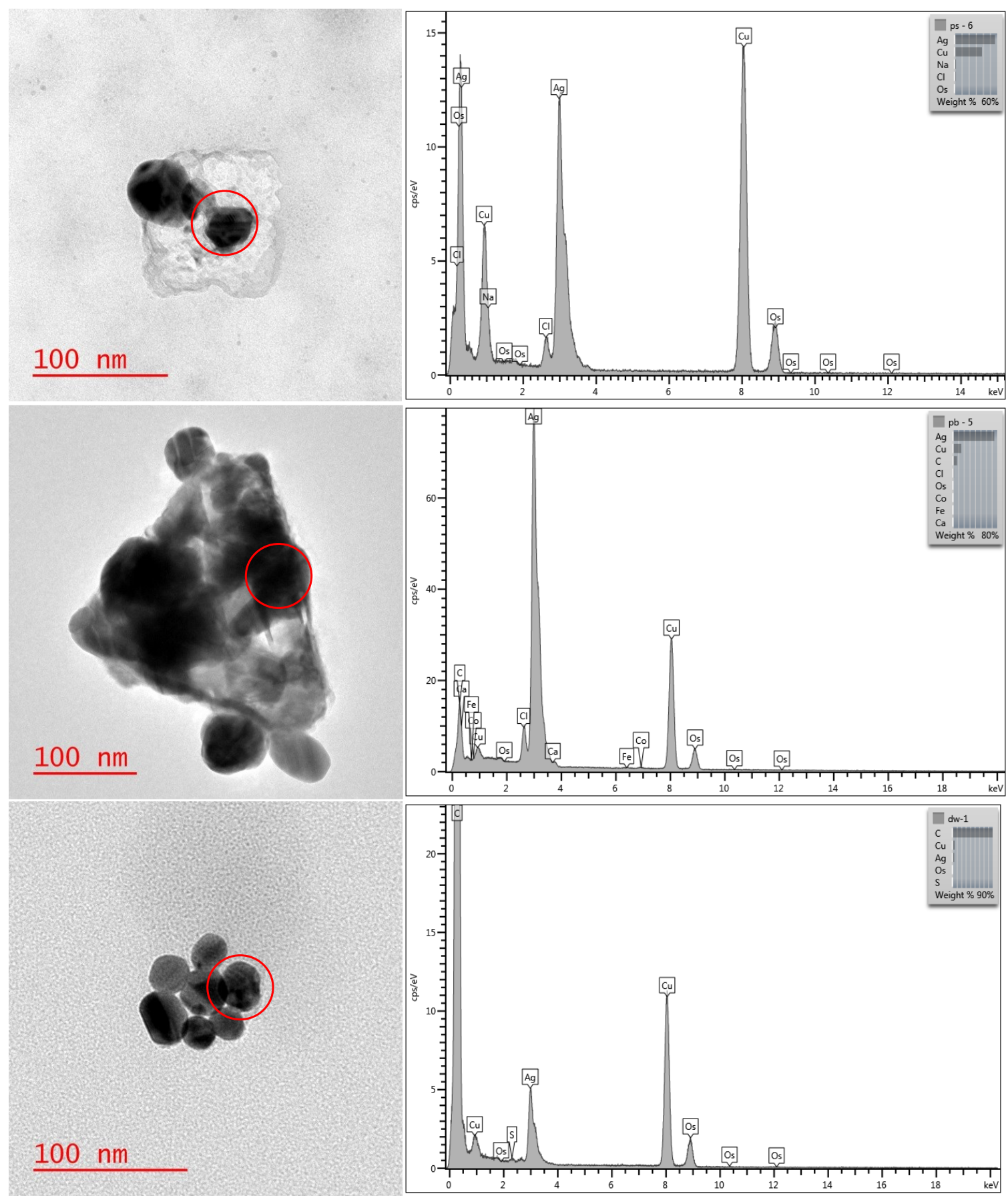

Figure S 3-2: TEM image and EDS data of pristine NPs weathered with a) sweat b) bleach and c) detergent. Circles indicate areas probed for EDS. 


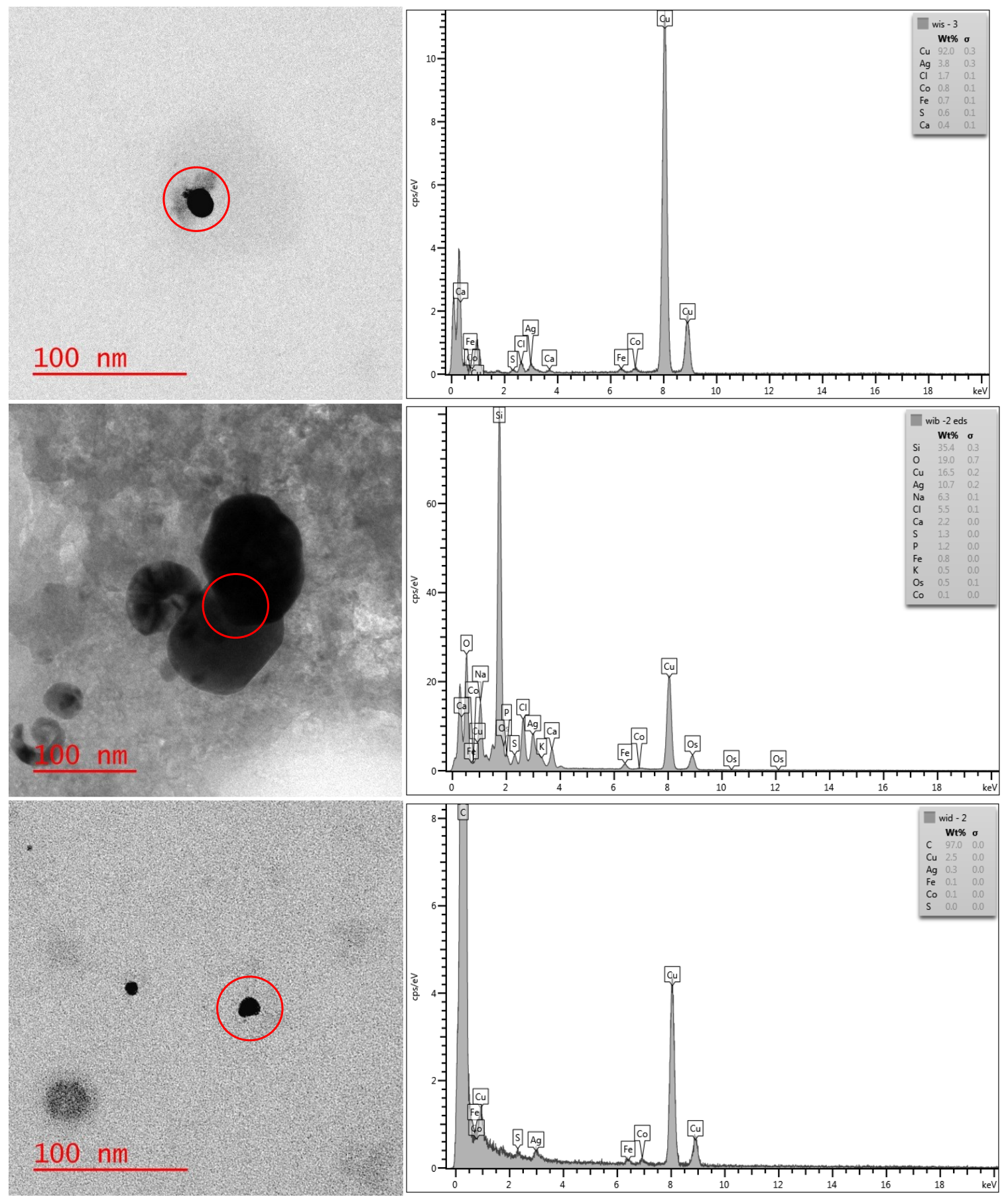

Figure S 3-3: TEM image and EDS data of extracted samples from a) sweat b) bleach c) detergent solutions after treatment with wastewater. Circles indicate areas probed for EDS. 

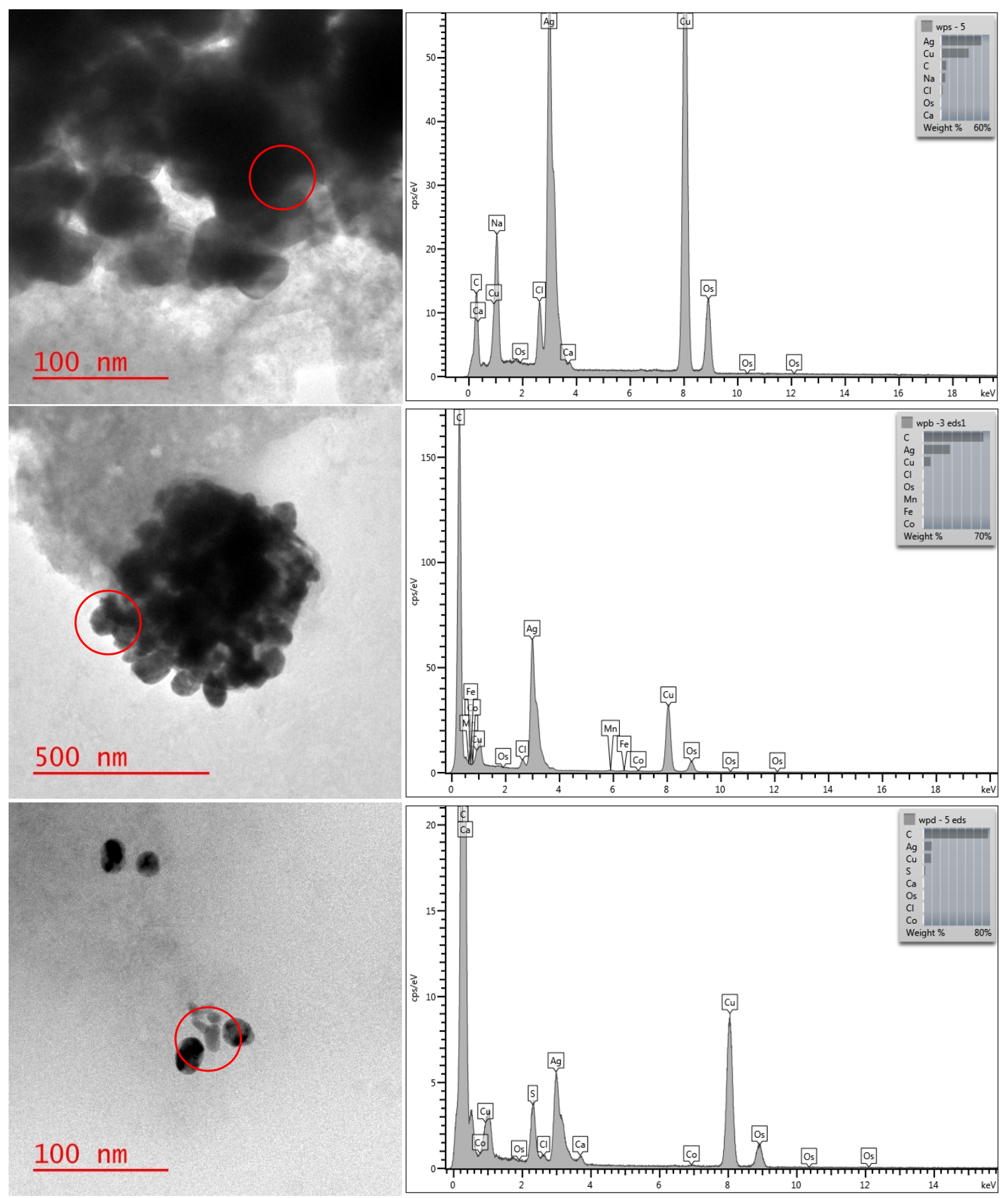

Figure S 3-4: TEM image and EDS data of pristine NPs treated with wastewater after weathering with a) sweat b) bleach c) detergent. Circles indicate areas probed for EDS. 


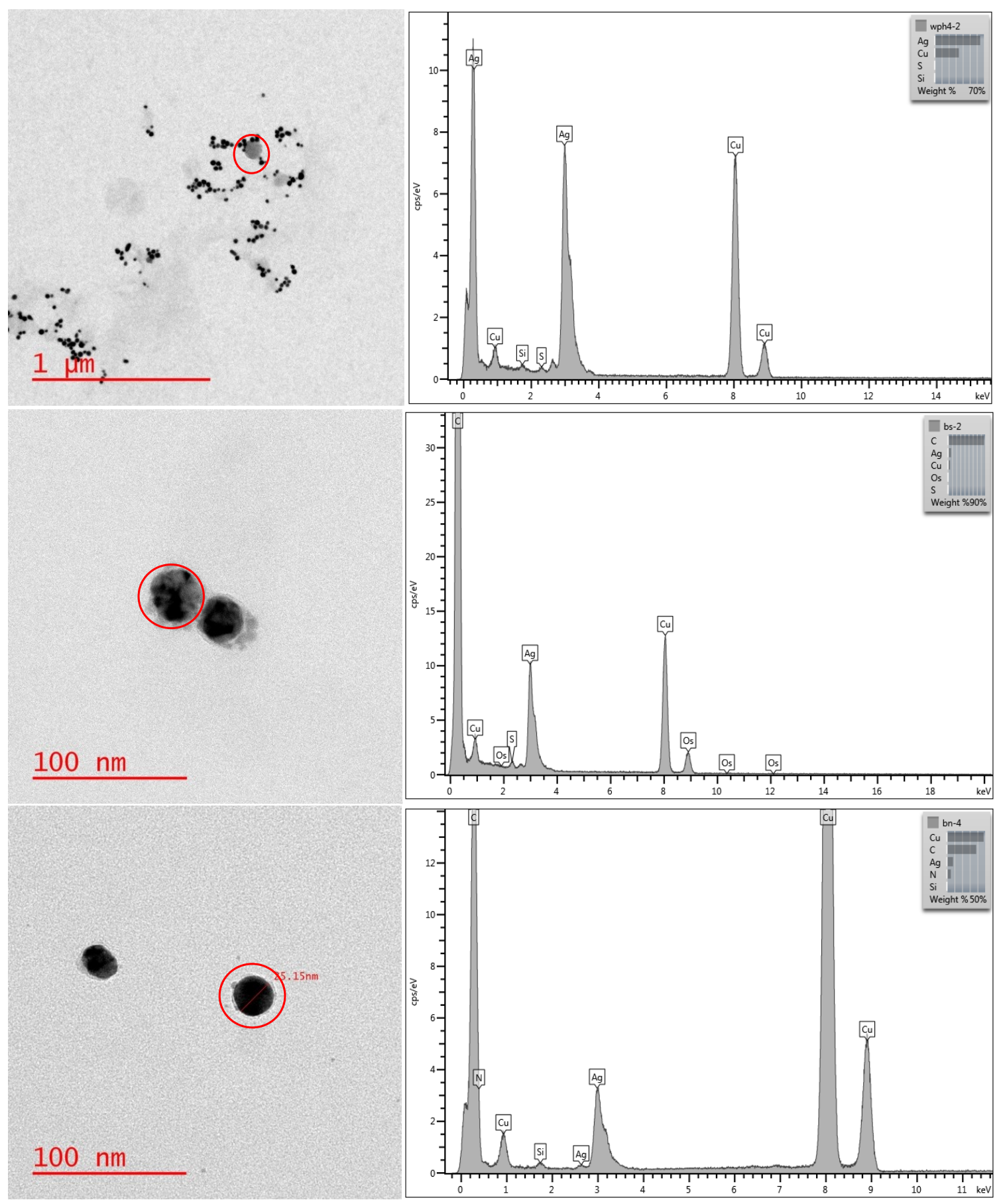




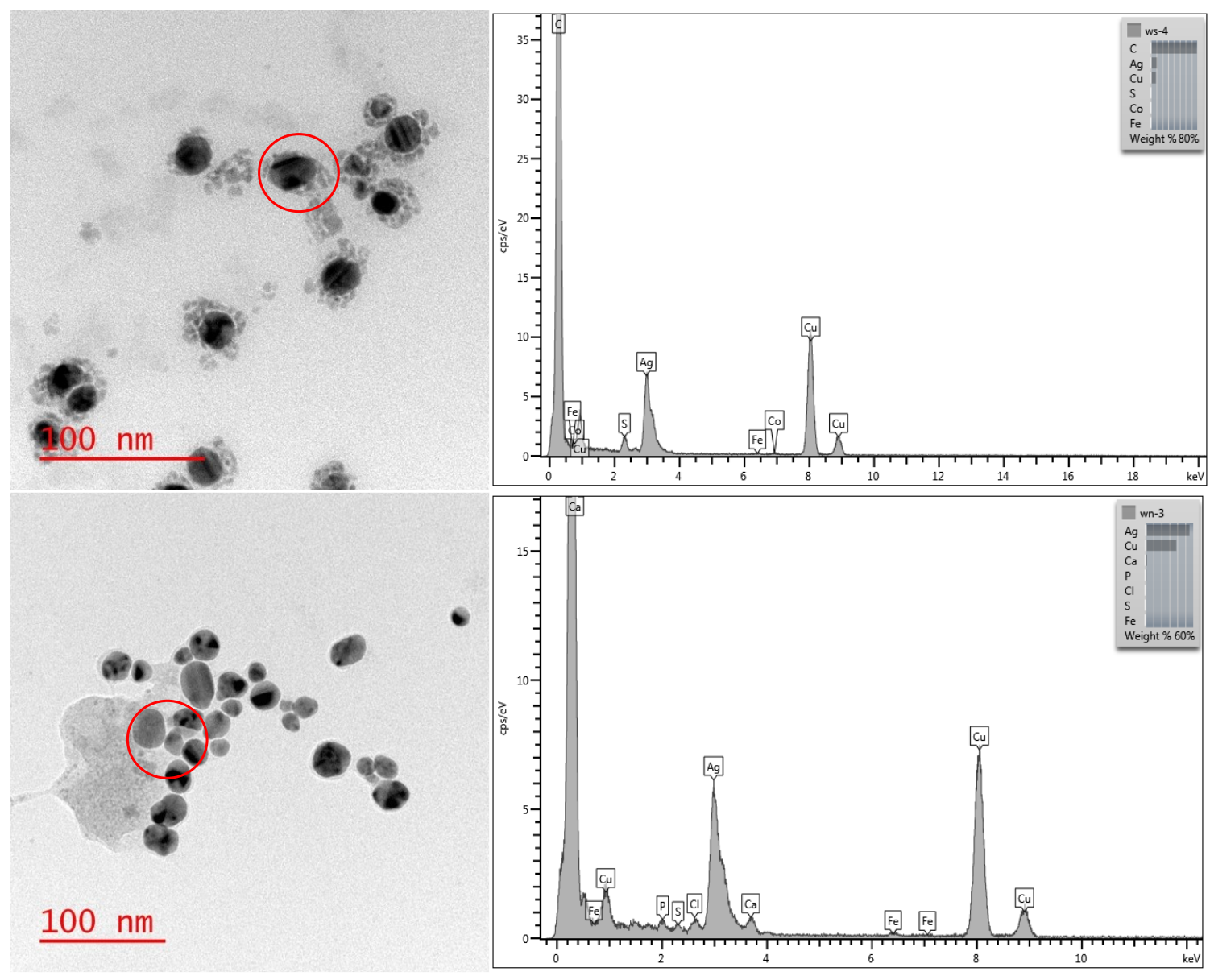

Figure S 3-5: TEM image and EDS data of pristine AgNPs in a) wastewater b) sulfurrich solution c) nitrogen-rich solution d) wastewater + sulfur-rich solution e) wastewater + nitrogen-rich solution. Circles indicate areas probed for EDS. 


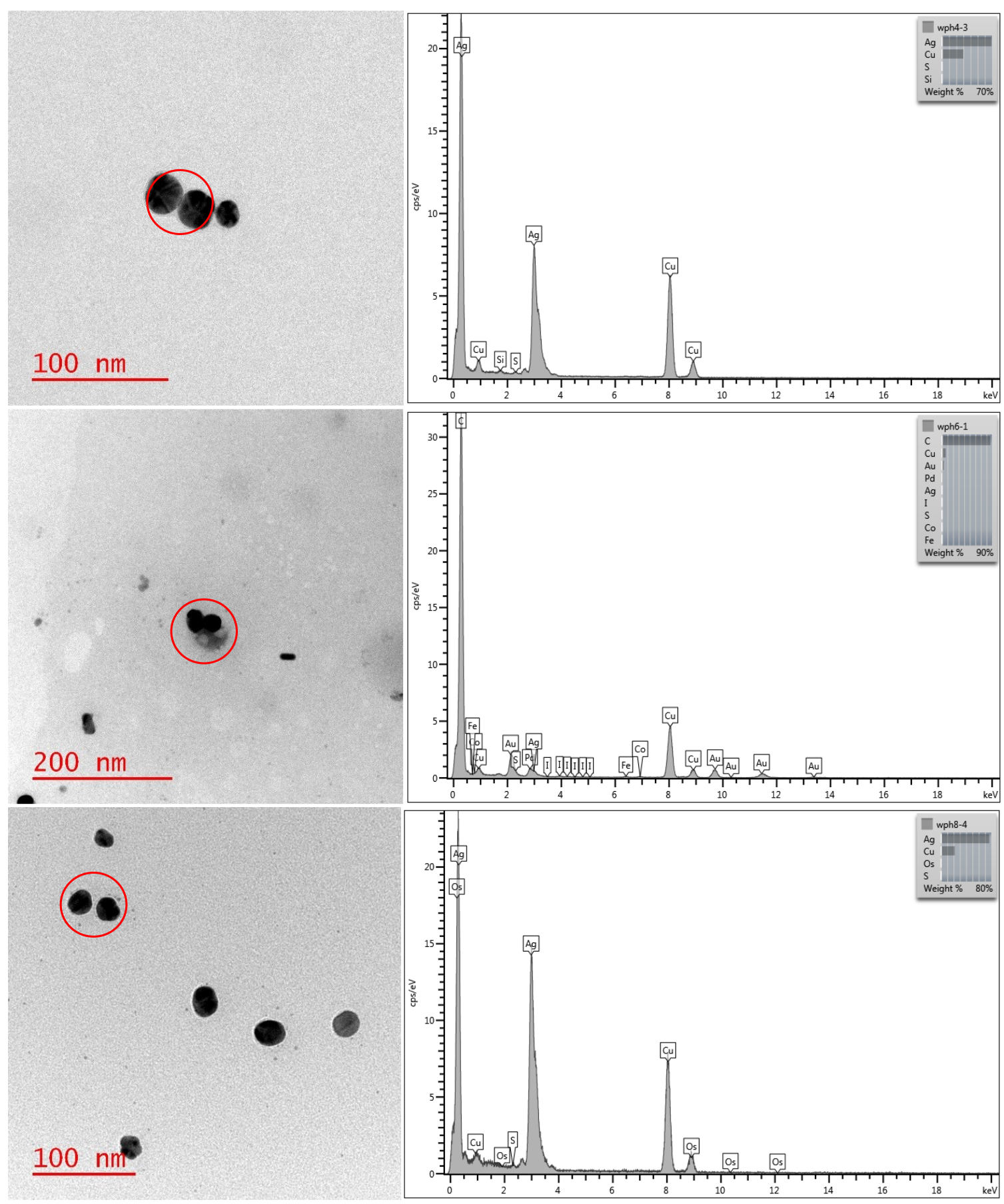

Figure S 3-6: TEM image and EDS data of pristine AgNPs in wastewater with $\mathrm{pH}$ altered to a) $\mathrm{pH} 4.0$ b) $\mathrm{pH} 6.0 \mathrm{c}$ ) $\mathrm{pH}$ 8.0. Circles indicate areas probed for EDS. 


\section{Chapter 4: Release and Transformation of Zinc Oxide Nanoparticles from Commercial Products Throughout Their Life Cycle}

Selvan Mohan, Kelsey Phillips, Juliska Princz, Maria C. DeRosa.

\subsection{Abstract}

Zinc Oxide nanoparticles (ZnO NPs) are used in commercial products for their ability to absorb UV light and for their antimicrobial activity. ZnO NPs can be found in sports clothing, UV protective clothing, sunscreen, and medicated baby powder. The widespread use of $\mathrm{ZnO}$ NPs can be expected to lead to increased concentrations within the environment. To accurately understand the effects of ZnO NPs on the environment, the inevitable transformation of these nanoparticles throughout their life cycle must be considered. The effects of sweat from contact with human skin, chlorine from swimming pool water, ultraviolet rays from sun exposure, and detergent and bleach from laundering were explored as potential use scenarios. The ZnO NPs were later introduced to wastewater to represent the path taken after release from its intended products. Inductively coupled plasma-mass spectrometer (ICP-MS), transmission electron microscopy (TEM), and energy dispersive X-ray spectroscopy (EDS) were used to analyze the release and transformation of these materials. ZnO NPs were observed to readily dissolve under acidic conditions. This process is accelerated in an acidic environment. Moreover, salts of $\mathrm{ZnCl}_{2}$ and $\mathrm{ZnS}$ are observed to form after the various treatments. 


\subsection{Introduction}

Nanoparticles, with their unique and often advantageous properties, have become integrated into many of our commercial products ${ }^{1}$. Zinc Oxide nanoparticles (ZnO NPs), in particular, have been employed within sunscreens ${ }^{2}$ for their UV protection, as well as within medicated baby powder and sports clothing for their antimicrobial properties ${ }^{3}$. The widespread use of ZnO NPs will inevitably lead to their introduction and accumulation in the environment. In fact, $\mathrm{ZnO}$ NP concentration found in agricultural soils treated with sewage sludge has been found to be higher than silver nanoparticles, another material that is found almost ubiquitously within nano-enabled products ${ }^{4}$.

There are many ways to synthesize $\mathrm{ZnO} \mathrm{NPs}^{5,6,7}$, as well as ways to deposit them onto a surface or integrate them into a product ${ }^{8}$. Wet deposition of $\mathrm{ZnO}$ NPs on a polymer or natural fiber leads to weak association with the surface, allowing for the nanoparticles to easily be removed from the fabric by simple water washes ${ }^{9}$. This would, of course, lead to the question of the effects these nanoparticles might have if they are released into the environment.

Numerous studies have looked at $\mathrm{ZnO}$ toxicological effects, such as on E.coli colonies, zebrafish embryos, and mammalian cells such as macrophages and epithelial cells $^{10,11,12}$. ZnO NPs have the ability to kill bacteria in a size-dependent fashion (within the range of $5-80 \mathrm{~nm}$ ) due to their ability to disrupt the cell membrane of even grampositive bacteria ${ }^{3,13,14}$. The dissolution of ZnO NPs also contributes towards their toxicity. The formation of ionic $\mathrm{Zn}^{2+}$ during the $\mathrm{ZnO}$ dissolution creates ROS that leads to the destruction of cells ${ }^{15,16}$. 
It is important to help understand the effects of nanoparticles on biological organisms, but ideally, the system under study should mimic what would actually be present within that biological system. Many toxicological studies employ purely pristine nanoparticles. Whether they were synthesized within the lab or purchased from a commercial source, these $\mathrm{ZnO}$ NPs may not be an accurate representation of the nanoparticle morphology and composition that would be likely introduced into the environment.

Nanoparticles have been shown to aggregate and agglomerate under non-pristine conditions, altering their morphology and perhaps even their behavior ${ }^{17}$. ZnO NPs can be transformed through their use: from their incorporation into the product matrix, their contact with sweat or other bodily fluids, detergents and bleaches, as well as weathering from the environment ${ }^{18,19}$. Moreover, these nanoparticles could be washed off the product or the user and find their way into the wastewater collection stream and, eventually, the treatment plant; both chemically complex environments could support further transformation.

ZnO NPs incorporated into textiles will come into contact with bleach, detergent, and other solutions present in a standard washing cycle, which may not only alter the nanoparticles themselves but also allow them leach from the fabrics ${ }^{20}$. Other weathering processes might involve contact from chlorine disinfected pools or simply contact with sweat, easily introduced by the consumers themselves (e.g., nano-enabled sunscreen or powder applied on the skin). Furthermore, the $\mathrm{ZnO}$ NPs could be altered by exposure to UV-radiation given that they are employed for their ability to block these rays ${ }^{21}$. In this paper, the morphology and composition of $\mathrm{ZnO}$ NPs are compared throughout a variety 
of treatments meant to mimic use scenarios in the lifecycle of the nanomaterial from

product incorporation up to right before the wastewater treatment process (Figure 4-1).

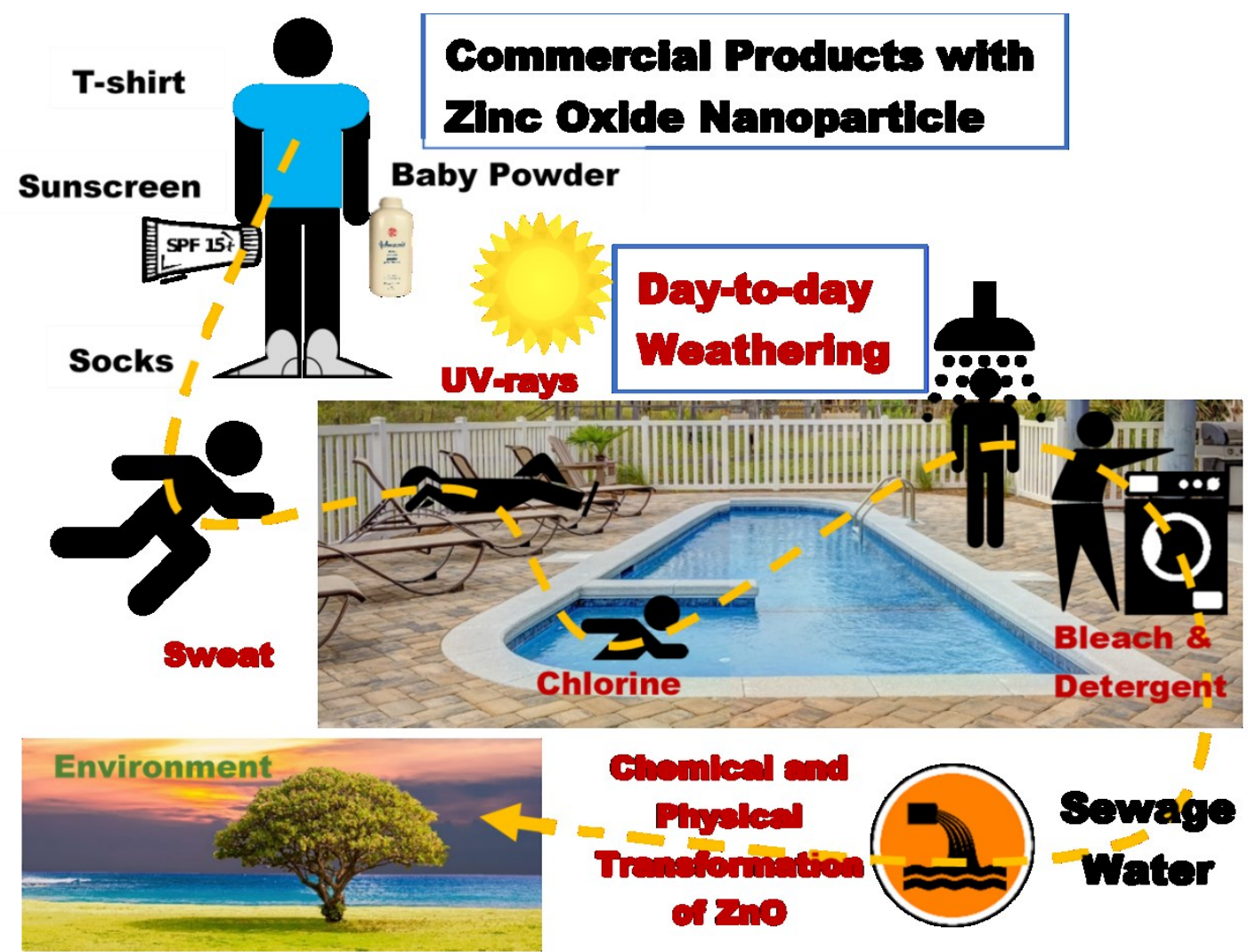

Figure 4-1: Overview of the path of potential transformation for $\mathrm{ZnO}$ nanoparticles studied in this paper.

\subsection{Materials and Methods}

Socks with ZnO nanoparticles (Sports Socks) were purchased from Nanosilver.

White Hanes sport cut socks, White George Men’s Short-Sleeve Basic T-shirt, Johnson's ${ }^{\circledR}$ baby powder, Clorox ${ }^{\circledR}$ bleach, and Tide ${ }^{\circledR}$ Original laundry detergent were purchased from Walmart. Johnson's ${ }^{\circledR}$ medicated baby powder was purchased from drugstrore.com. White Coolibar Long Sleeve T-shirt XXL was purchased from 
coolibar.com. Aveeno Active Naturals sensitive skin mineral sunscreen (SPF 50), Aveeno Baby sunscreen lotion (SPF 55), Garnier Ombrella Kids hypoallergenic lotion (SPF 50+), and Neutrogena Ultra Sheer face sunscreen (SPF 60) were purchased from Shoppers Drug Mart. Exo-terra Sunray metal halide fixture with ballast and bulb (70 W), as well as a Large Zilla Fresh Air screen habitat, were purchased from bigalspets.ca. Raw wastewater was obtained from the Robert O. Pickard Environmental Centre wastewater plant (Ottawa, Ontario, Canada). TEM grids were purchased from Electron Microscopy Sciences. All acids used were ultrapure reagent grade unless specified. Only Milli-Q double deionized water was used in this experiment. All other chemicals were purchased from Sigma Aldrich. All glassware was cleaned with aqua-regia before used.

\subsubsection{ZnO nanoparticle synthesis}

$200 \mathrm{~mL}$ of $0.2 \mathrm{M}$ zinc acetate dihydrate, $\mathrm{Zn}\left(\mathrm{O}_{2} \mathrm{CCH}_{3}\right)_{2}\left(\mathrm{H}_{2} \mathrm{O}\right)_{2}$ solution was prepared in a $1 \mathrm{~L}$ round bottom flask. $200 \mathrm{~mL}$ of $0.5 \mathrm{M}$ sodium hydroxide, $\mathrm{NaOH}$ solution was prepared in a $500 \mathrm{~mL}$ round bottom flask. Both solutions were heated to 60 ${ }^{0} \mathrm{C}$ in separate oil baths. The $\mathrm{Zn}\left(\mathrm{O}_{2} \mathrm{CCH}_{3}\right)_{2}\left(\mathrm{H}_{2} \mathrm{O}\right)_{2}$ solution was stirred vigorously for 15 minutes once the desired temperature was reached. Next, the heated $\mathrm{NaOH}$ solution was quickly added ( $<3$ seconds) to the $\mathrm{Zn}\left(\mathrm{O}_{2} \mathrm{CCH}_{3}\right)_{2}\left(\mathrm{H}_{2} \mathrm{O}\right)_{2}$ and left to stir for 1 hour at 60 ${ }^{0} \mathrm{C}$. Then the solution was allowed to cool to room temperature without stirring ${ }^{22}$. A murky white solution was observed. 
The nanoparticle suspension was then transferred to $8 \times 50 \mathrm{~mL}$ centrifuge tubes. These tubes were centrifuged at $10000 \mathrm{rpm}$ for $10 \mathrm{mins}$. The supernatant was discarded and replaced with the same volume of water. The pellet was re-suspended into solution by sonicating for 10 mins along with vigorous periodical shaking. The centrifuge tubes are centrifuged again. These steps are repeated until the supernatant was clear to sight after centrifugation (3-5 times) $)^{22}$.

The particles (solid white pellet) were left to dry for 12 hours. Once dry, the particles were ground manually into a fine white powder using a pestle and $\operatorname{mortar}^{22}$. The powder was left again to dry for 12 hours, giving a $92 \%$ yield of ZnO NPs. The nanoparticles were characterized using TEM and EDS after resuspension in water.

\subsubsection{Nanoparticle Extraction}

\subsubsection{Incineration}

Consumer products were incinerated to isolate the metal contained within them. A pre-weighed amount of consumer product sample $(\sim 100 \mathrm{mg})$ was placed in a $10 \mathrm{ml}$ Pyrex beaker. The beaker was placed into a TEMCO 1520 benchtop muffle furnace at $600{ }^{\circ} \mathrm{C}$ and left for 3 hours or until the sample turned to white ash. The sample was left to cool to room temperature before being removed from the furnace ${ }^{23}$. The ash was dissolved in 1 $\mathrm{mL}$ of $\mathrm{H}_{2} \mathrm{O}$ and $10 \mu \mathrm{L}$ of $\mathrm{HNO}_{3}$ before any analysis was done. 


\subsubsection{Solvent washing}

Consumer products were washed with various polar and non-polar solvents to isolate the metal nanoparticles within the product. 2-3 $\mathrm{g}$ of consumer product sample was added into a $50 \mathrm{~mL}$ centrifuge tube along with $30 \mathrm{~mL}$ of hexane. The solution was sonicated and vortexed until the sample was dissolved. The sample was then centrifuged using Sorvall Legend XIR Centrifuge at $7000 \mathrm{rpm}$ for 2 minutes. The supernatant was removed, and the washing process was repeated on the pellet with ethanol, water, and repeated aliquots of acetone until a clear supernatant was observed (3-5) ${ }^{24}$. The pellet was stored dry and dissolved into acetone when needed.

\subsubsection{Day-to-day use scenarios with bleach, chlorine, detergent, sweat.}

Day-to-day use scenarios were performed to detect the transformation and measure the leaching of nanoparticles from commercial products. $4 \mathrm{~g}$ of the consumer product containing nanoparticles was added to a $250 \mathrm{~mL}$ Erlenmeyer flask along with $100 \mathrm{~mL}$ of four respective solutions. $1.3 \mathrm{~mL}$ and $0.8 \mathrm{~mL}$ of bleach (Clorox), and detergent (Tide Original) respectively were added to $100 \mathrm{~mL}$ of $\mathrm{ddH}_{2} \mathrm{O}$ each to model typical laundry concentrations ${ }^{25} .5 .8 \mu \mathrm{L}$ chlorine $(60 \% \mathrm{NaClO})$ was added to $100 \mathrm{~mL}$ of $\mathrm{H} 2 \mathrm{O}$ to model a pool water system ${ }^{26}$. For the sweat solution, a $1.08 \% \mathrm{NaCl}, 0.12 \%$ lactic acid, and $0.13 \%$ urea solution was made ${ }^{23}$. This was then placed on an Innowa 40 Incubator Shaker Series at 100 oscillations per minute at $37^{\circ} \mathrm{C}$. This was left for 24 hours to mimic the wear the sock will go through during multiple uses. The artificial sweat, 
chlorine, detergent, and bleach solution were $\mathrm{pH} 3^{27}, \mathrm{pH} 10, \mathrm{pH} 9$, and $\mathrm{pH} 11$, respectively.

\subsubsection{UV Exposure}

Consumer products were exposed to UV radiation to mimic exposure to the sun. $\mathrm{ZnO}$ samples were separately exposed to UV light. The sock and T-shirt were exposed to full-spectrum UV light (280-1000 nm wavelength) for 7 days in 12 hours/day cycles. The sunscreen, powder and synthesized $\mathrm{ZnO}$ nanoparticles were continuously exposed to UV light for 24 hours. The samples were placed at the bottom of the terrarium, 0.6 meters below the light, exposing the sample to 19600 lux of illuminance. The samples were only placed in a small area directly under the lamp to ensure maximum and even exposure to the UV light. These samples were then exposed to day-to-day use.

\subsubsection{Wastewater exposure}

Leached nanoparticles from consumer products obtained after day-to-day use scenarios were mixed in at 1:1 weight ratio with raw wastewater for a total mass of $2.0 \mathrm{~g}$. These mixtures were then vortexed on a Vortex Genie 2, Scientific Industries for 2 minutes and left for 24 hours. The mixture was vortexed again before the TEM grids were prepared.

The raw wastewater was used within 6 hours of collection from the sewage treatment plant, and the content of raw wastewater was analyzed within 48 hours. Sewage water content can be found in supporting information (Table S4-1) 


\subsubsection{Analysis}

The TEM images were taken using FEI Tecnai G2 TEM at $200 \mathrm{kV}$. The solutions were then vortexed for 2 minutes. $6 \mu \mathrm{L}$ of the sample was placed on the grid and left for a minute. Residual liquid was removed and another $6 \mu \mathrm{L}$ of the sample was added. The residual sample was removed for a second time, and the grid was left to air dry for 2 hours. The CF300-Cu grid was used.

The EDS of the sample was taken through an attachment to the TEM, Aztec EDS, from Oxford X-ray detection systems. The beam was made to focus directly on the nanoparticle.

ICP-MS was analyzed on a Varian 820-MS (Varian, Australia now Analytik-Jena, Germany). The solutions were diluted appropriately to have a metal concentration within the standard curve. $0.1 \%$ aquaregia solution was run between samples to ensure no compounding of the signal is observed.

\subsection{Results and Discussion}

Out of the numerous commercial products containing $\mathrm{ZnO}$ NPs, four products were chosen based on local availability. A control product with no indicated $\mathrm{ZnO}$ content was also analyzed for comparison in each type. Pristine ZnO NPs were also synthesized to compare their behavior to the nanoparticles contained in the commercial products when exposed to different environmental factors. 
The various sources containing $\mathrm{ZnO}$ NPs will be here on referred to as socks, tshirt, powder, sunscreen, and pristine nanoparticles. The control sources will be referred to as respective controls. The samples above were analyzed for the presence of ZnO NPs using TEM and EDS. Figure 4-2 below shows the TEM image and EDS data for the $\mathrm{ZnO}$ NP containing sources. Samples for socks, t-shirt, powder, and sunscreen were prepared by incinerating the sample and then extracting the nanoparticles. The synthesized nanoparticles were analyzed with no further modification. 


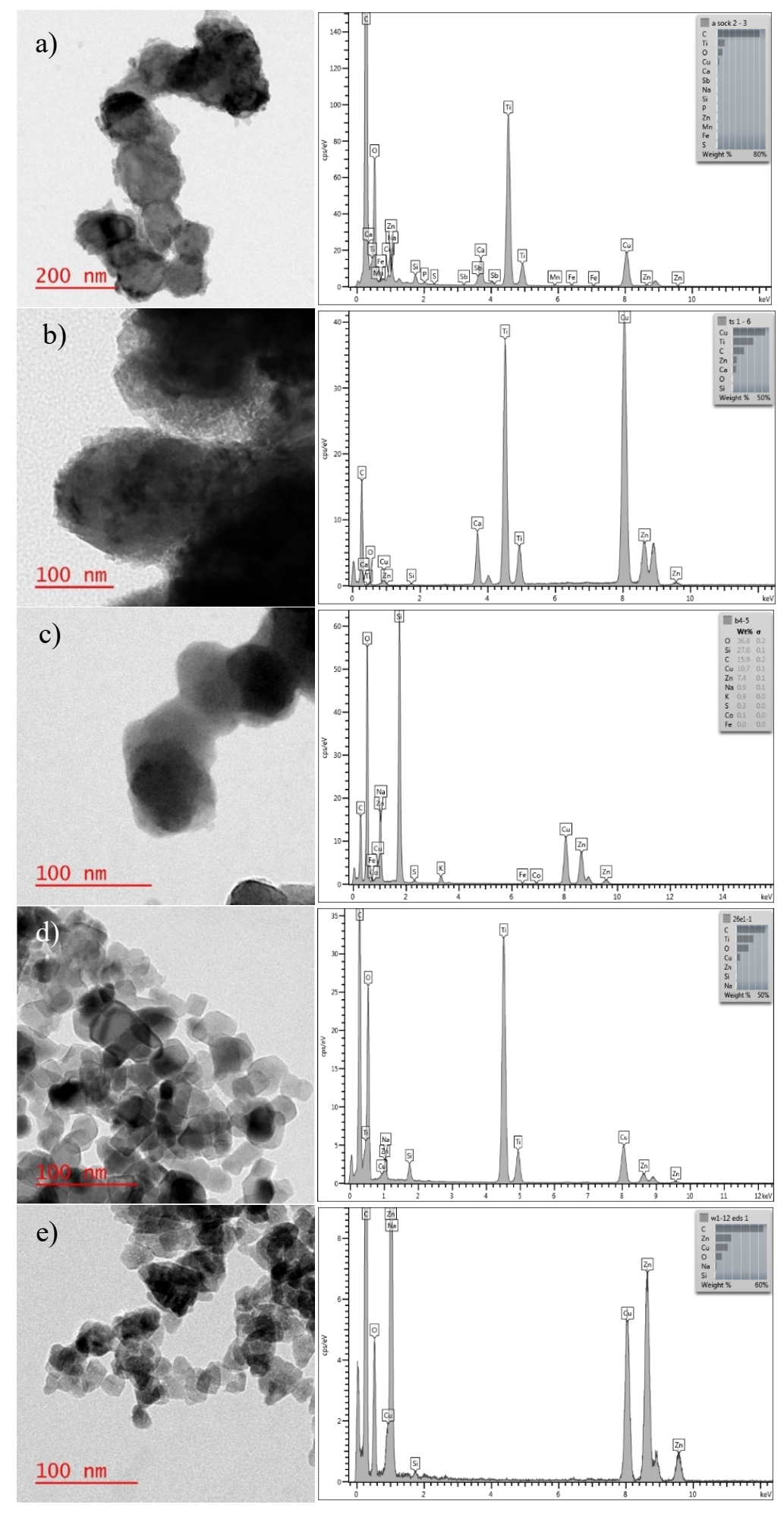

Figure 4-2: TEM images and EDS spectrum of ZnO NPs of sources: a) White Nanosilver Sports socks, b) White Coolibar Long Sleeve T-shirt, c) Johnson's medicated baby powder, d) Aveeno Active Naturals sensitive skin mineral sunscreen SPF 50 and e) Labsynthesized pristine nanoparticles. Samples were incinerated for the extraction of nanoparticles. 
Nanoparticles were also extracted through washes of hexane, ethanol, water and acetone as shown in Figure 4-3

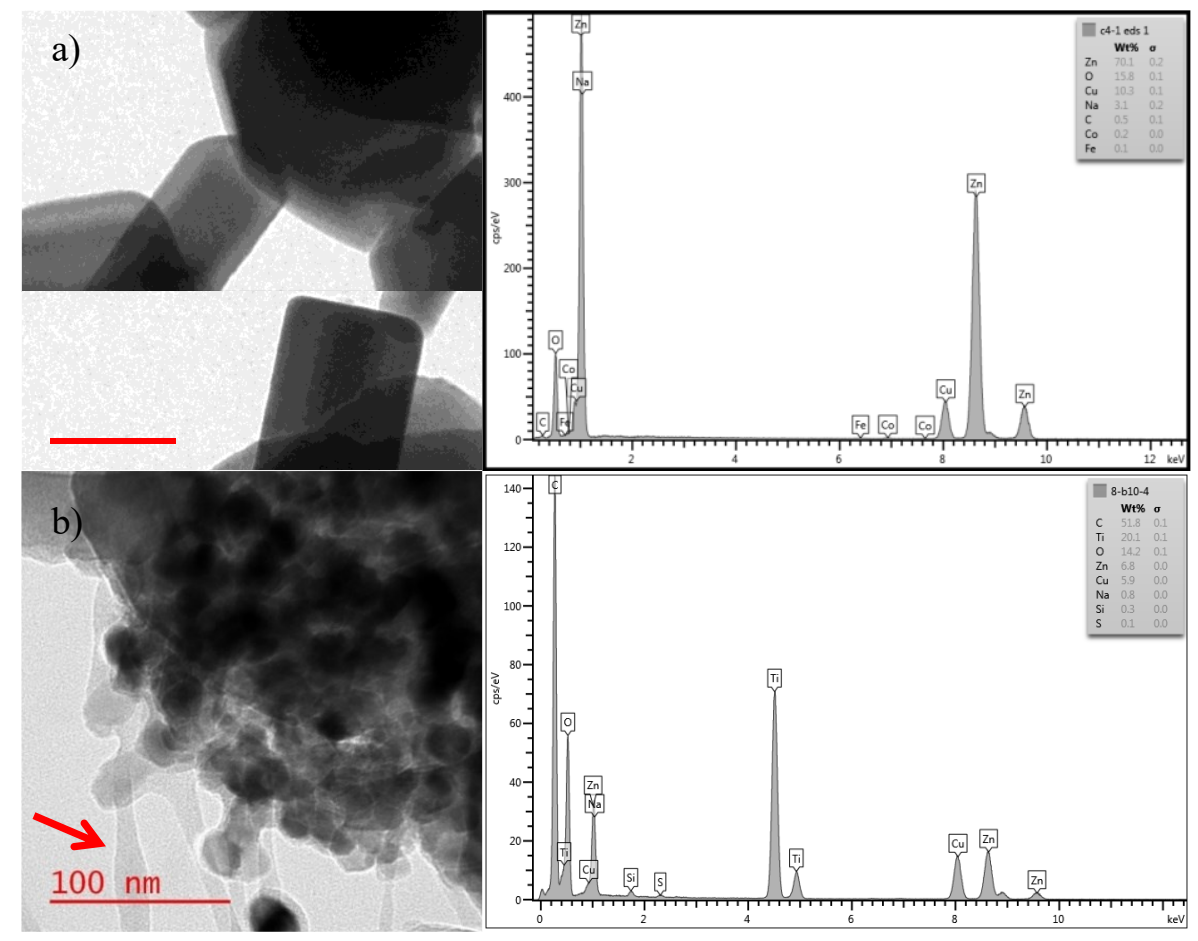

Figure 4-3: TEM images EDS spectrum of ZnO NPs of sources, a) Johnson's

medicated baby powder b) Aveeno Active Naturals sensitive skin mineral sunscreen SPF 50. Samples were extracted through the solvent washing process.. The solvent washing extraction method was applied to powder and sunscreen. Nanoparticles were observed to be physically and chemically similar to those extracted via incineration (Figure 4-2). However, there is an irregular shape observed around the nanoparticles extracted through the solvent extraction process, indicated by the arrow in Figure 4-3. Upon performing EDS analysis on the irregular shape, it was determined that the irregular shapes consist of $\mathrm{ZnO}$ NPs that are dissolving into the solution and drying on the grid as a thin film ${ }^{28-30}$. This dissolution pattern of $\mathrm{ZnO}$ NPs is observed in other data presented throughout this study. 


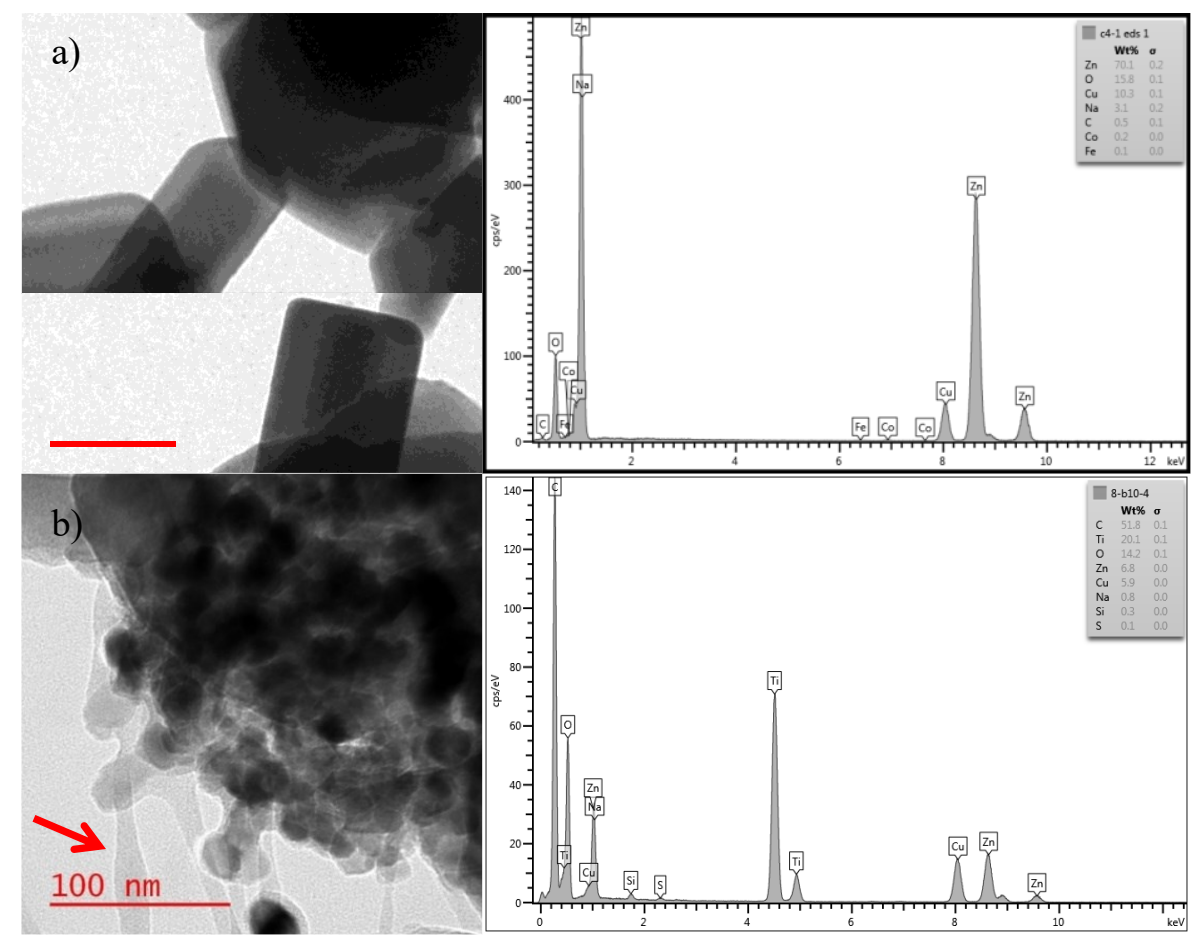

Figure 4-3: TEM images EDS spectrum of ZnO NPs of sources, a) Johnson's medicated baby powder b) Aveeno Active Naturals sensitive skin mineral sunscreen SPF 50. Samples were extracted through the solvent washing process.

The $\mathrm{ZnO}$ NPs in the socks, $\mathrm{t}$-shirt, and sunscreen were found together with titanium dioxide nanoparticles $\left(\mathrm{TiO}_{2} \mathrm{NPs}\right)$. These two nanoparticles were needed to provide complete UV protection through the UVA and UVB spectrum ${ }^{31}$. $\mathrm{TiO}_{2} \mathrm{NPs}_{\text {are }}$ also used to give the white color to socks and t-shirt ${ }^{32}$. TEM and EDS analysis were done on BEHR Ultra white paint, which showed the presence of $\mathrm{TiO}_{2} \mathrm{NPs}_{\text {s }}$ used to obtain a white color (Figure S 4-1, Supporting information).

$\mathrm{ZnO}$ NPs are far more soluble in water than $\mathrm{TiO}_{2} \mathrm{NPs}^{33,34}$. The high solubility would cause the structure of the $\mathrm{ZnO}$ NPs to change due to dissolution during product storage, life cycle, and sample preparation. The images above (Figure 4-2 and Figure 4-3) show the sample contains both $\mathrm{ZnO}$ and $\mathrm{TiO}_{2}$ NPs at the same location. This could 
be due to the $\mathrm{ZnO}$ precipitating on the surface of the $\mathrm{TiO}_{2}$ when the sample is dried on the TEM grid. In the powder, $\mathrm{Zn}$ was observed to have precipitated around the silica present in the talc (Figure $\mathbf{S} \mathbf{4 - 2}$, supporting information).

It can also be noted that the synthesized nanoparticles have a similar physical appearance to those in sunscreen. This could be because the nanoparticles in sunscreen are mixed with the product post-synthesis. Meanwhile, $\mathrm{ZnO}$ NPs are likely synthesized in situ on fabrics (socks and t-shirt) or on silica (powder). In situ synthesis ensure adhesion of the nanoparticles to the material ${ }^{35}$. Moreover, to aid with synthesis, cotton and silica could serve as nucleation sites for the formation of $\mathrm{ZnO}$ nanoparticles ${ }^{36}$.

Commercial products with no ZnO NPs were analyzed as controls. Figure 4-4 below shows the various control products and their methods of extraction. TEM images of powder and sunscreen extracted via incineration can be found in supporting information (Figure S 4-3). It was found that the control sock and sunscreens have $\mathrm{TiO}_{2}$ nanoparticles due to the desired white pigmentation ${ }^{32}$. 


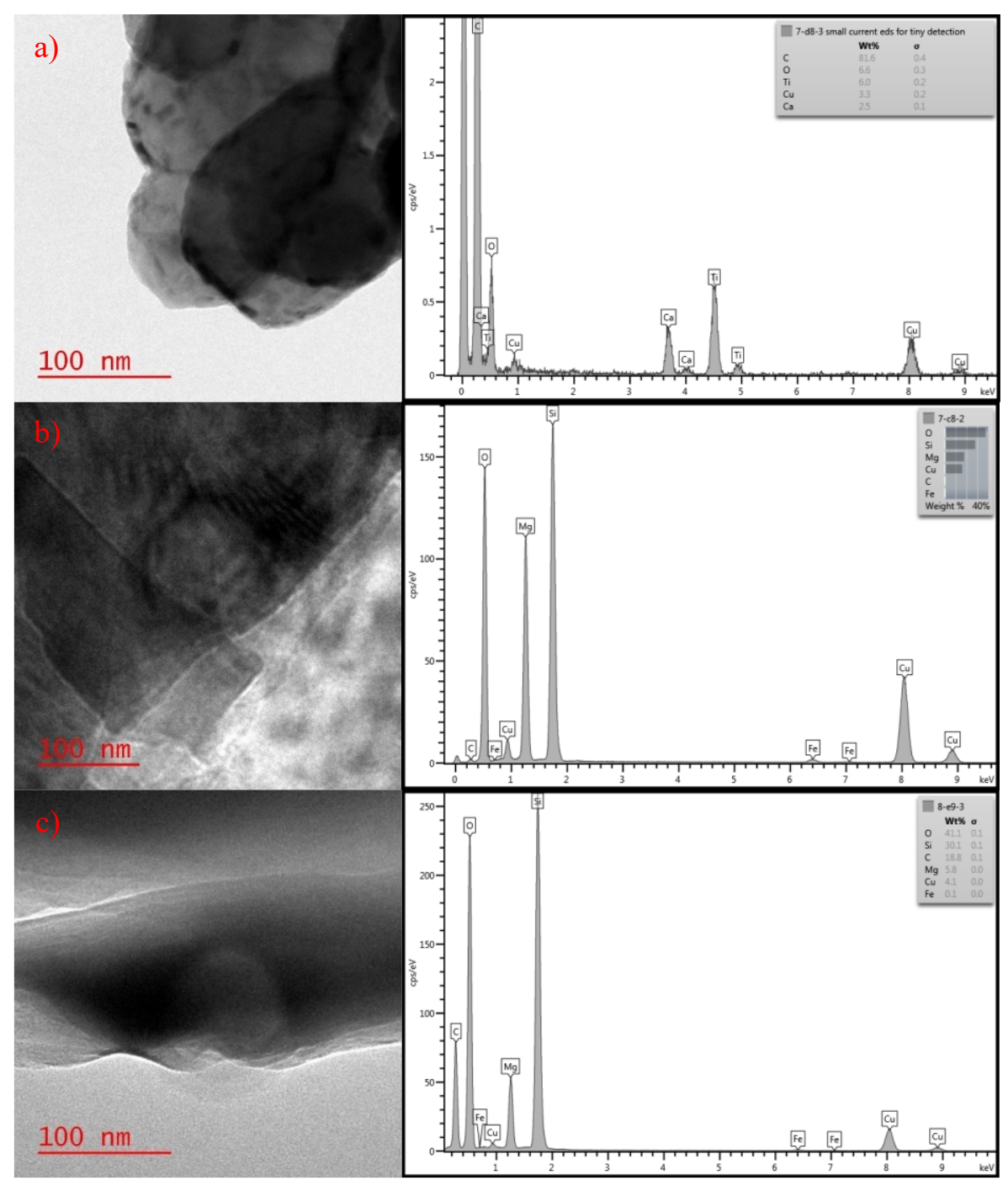




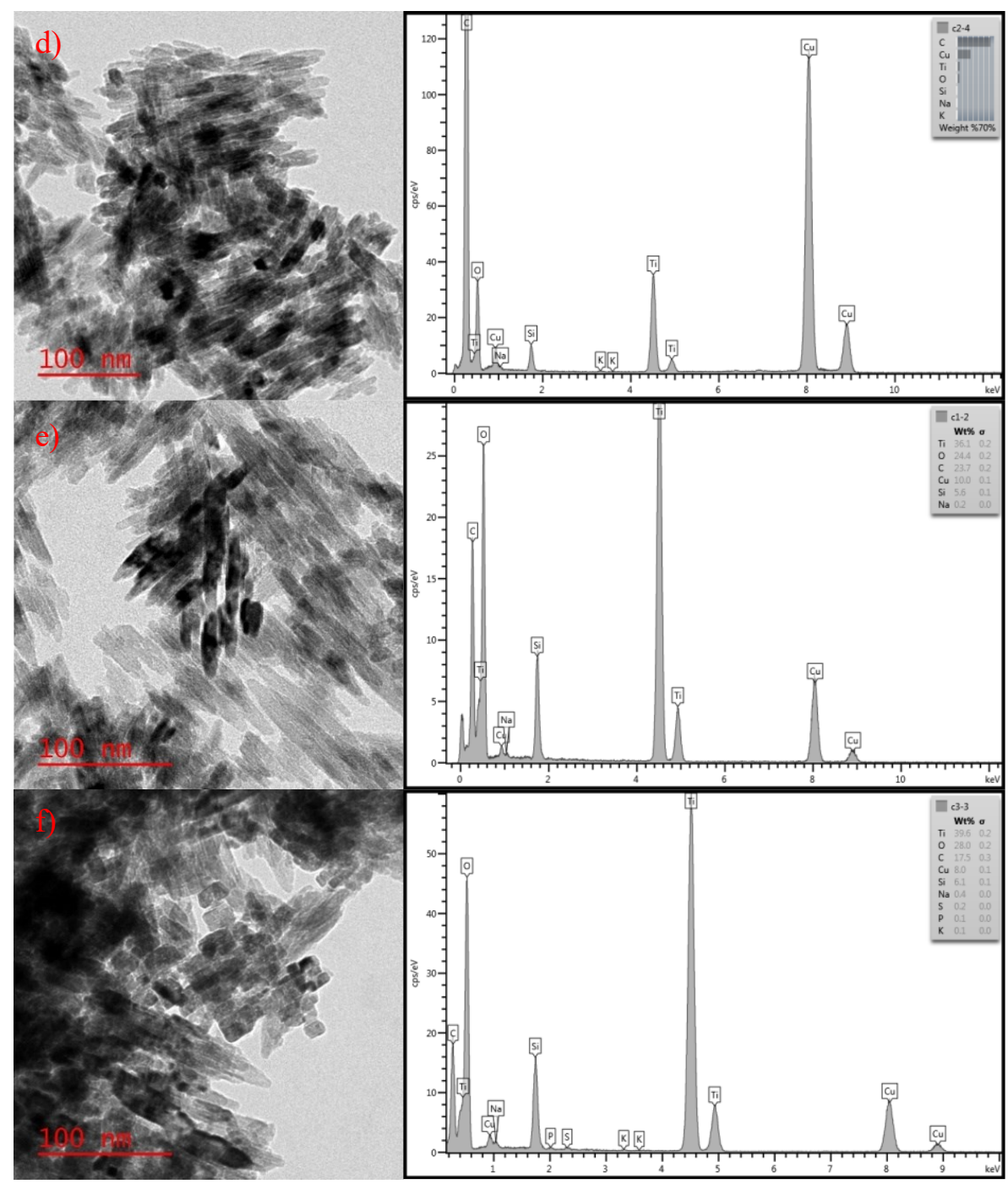

Figure 4-4: TEM images and EDS spectrum of control sources: a)White Hanes Sports Cut Socks (incineration), b)White George Men's Short-Sleeve Basic T-shirt (incineration) c) Johnson's baby powder (solvent washing) d)Aveeno Baby sunscreen lotion SPF 55 (solvent washing), e)Garnier Ombrella Kids hypoallergenic lotion SPF 50+ (solvent washing) and f)Neutrogena Ultra Sheer face sunscreen SPF 60. Samples were extracted through the solvent washing process.

The different commercial products containing nanoparticles were then put through day-to-day use conditions (sweating, chlorine from swimming pools, washing with detergent and bleaching) to observe leaching as well as the physical and chemical transformation that will occur. These solutions were analyzed using a TEM after 
incubating with the sample for 24 hours. TEM images are shown in Table 4-1 below. There were no TEM data presented for socks due to the low concentration of $\mathrm{ZnO}$ nanoparticles in the extracted solution making it impossible to locate on a TEM grid.

The dissolution of $\mathrm{ZnO}$ nanoparticles was observed throughout all samples. Irregular shapes around the nanoparticles were analyzed through EDS to confirm the presence of $\mathrm{Zn}$ hence supporting the theory of dissolution. It is also observed that the dissolution of $\mathrm{ZnO}$ nanoparticles was more pronounced in acidic conditions (e.g., sweat $)^{37}$.

In powder, $\mathrm{ZnO}$ nanoparticles were initially observed to be present on the surface of silica (Figure 4-2). However, looking at the powder sample treated with sweat in Table 4-1, individual nanoparticles resembling what was found in sunscreen and the pristine nanoparticles seemed to be more prevalent. The ZnO NPs in the powder may have dislodged or dissolved into the sweat solution and reprecipitated during the drying process on the TEM grid. The recrystallization of $\mathrm{ZnO}$ is observed later in Table 4-3, where the incubation of powder with raw wastewater was done. $\mathrm{ZnO}$ precipitating as nanoparticles when drying had been previously reported in literature ${ }^{38}$.

Association of $\mathrm{Zn}$ with $\mathrm{Cl}$ and $\mathrm{S}$ was observed throughout the samples in this paper. This suggests the formation of $\mathrm{ZnCl}_{2}$ and $\mathrm{ZnS}$ salts. It has been previously reported that $\mathrm{ZnO}$ does transform to $\mathrm{ZnS}$ during anaerobic digestion of the wastewater treatment process ${ }^{39} . \mathrm{Cl}$ and $\mathrm{S}$ on the surface of the nanoparticles will promote the dissolution of the nanoparticle causing $\mathrm{Zn}$ to dissolve into solution as they can form complexes with $\mathrm{Zn}^{2+}$ and instantly precipitate away from the particle ${ }^{29,40}$. 
Table 4-1: TEM images of nanoparticles extracted from commercial products after subjected to day-to-day use conditions for 24-hours. Please refer to supporting information (Figure S 4-4, Figure S 4-5, Figure S 4-6, and Figure S 4-7) for EDS spectra.

\begin{tabular}{|c|c|c|c|c|}
\hline $\begin{array}{l}\text { Use } \\
\text { Condition }\end{array}$ & Sweat & Chlorine & Detergent & Bleach \\
\hline T-shirt & & & & \\
\hline Powder & & & & \\
\hline Sunscreen & & & & \\
\hline $\begin{array}{l}\text { Pristine } \\
\text { Nanoparticles }\end{array}$ & & & & \\
\hline & & & & $100 \mathrm{~nm}$ \\
\hline
\end{tabular}

The various commercial products were also exposed to UV light before day-today use conditions were applied. The images after UV exposure can be found in Table 4-2. The UV exposure of the commercial products did not make much difference in the morphology of the released nanoparticles. The nanoparticles are of the same size and 
show similar aggregation and agglomeration patterns when compared to those without UV exposure. The aggregation can be observed by the clusters of closely associated nanoparticles while the agglomeration is observed by the larger particles that are forming. The EDS results showed $\mathrm{Cl}$ and $\mathrm{S}$ present alongside with $\mathrm{Zn}$. 
Table 4-2: TEM images of nanoparticles extracted from commercial products that had been subjected to day-to-day use conditions for 24-hours after being exposed to UV light for 24-hours. Please refer to supporting information (Figure S 4-8, Figure S 4-9, Figure S 4-10, and Figure $S$ 4-11) for EDS spectra.

\begin{tabular}{|c|c|c|c|c|}
\hline $\begin{array}{l}\text { Use } \\
\text { Condition } \\
\text { Sources }\end{array}$ & Sweat & Chlorine & Detergent & Bleach \\
\hline T-shirt & & & & \\
\hline Powder & & & & \\
\hline Sunscreen & & & & \\
\hline $\begin{array}{l}\text { Pristine } \\
\text { Nanoparticles }\end{array}$ & & & & \\
\hline
\end{tabular}

Running single particle-ICP-MS on ZnO NP samples did not provide conclusive particle size data due to very high background noise from the sample. The high noise is caused by $\mathrm{ZnO}$ dissolved in solution in ionic form (dissolution) when the sample is heavily diluted for single-particle analysis. SP-ICP-MS requires a low background signal 
to distinguish individual nanoparticles accurately ${ }^{41}$. Therefore, in Figure 4-5, the quantitative leaching of $\mathrm{ZnO}$ from commercial products was analyzed and compared with UV treatment using ICP-MS alone.

ICP-MS was run on the solutions used to simulate day-to-day conditions (the same solutions used for TEM). The data in Figure 4-5 represents the amount of $\mathrm{ZnO}$ leaching from $4.00 \mathrm{~g}$ of commercial product leaching into $100 \mathrm{ml}$ solutions of day-to-day use conditions over a 24-hour incubation. Do note that due to the low concentration of $\mathrm{Zn}$ in socks, ICP-MS results were presented in ppb. 


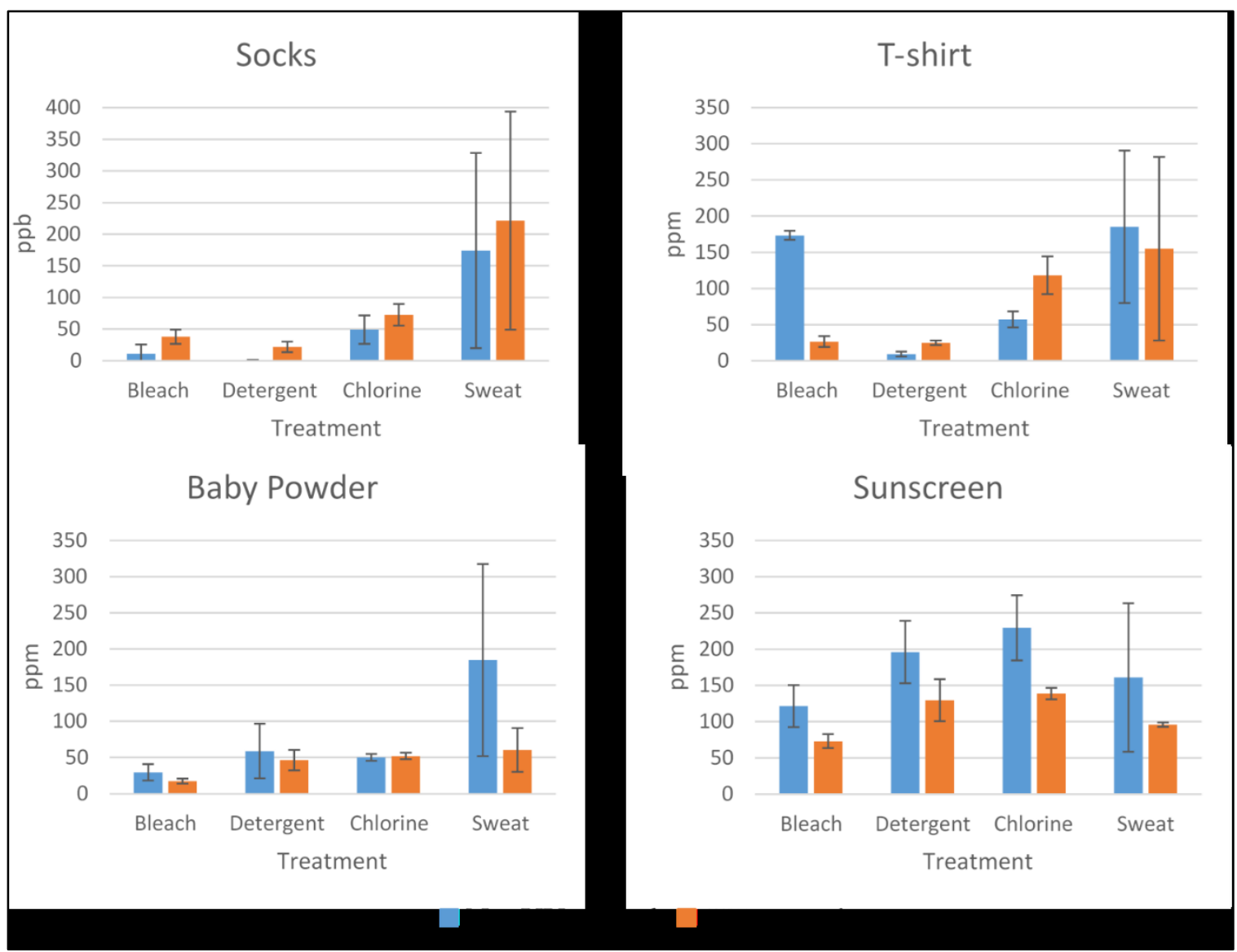

Figure 4-5: Leaching of nanoparticles from socks, t-shirt, powder, and sunscreen when incubated in bleach, detergent, chlorine, and sweat solution for 24-hours to mimic day-today use conditions with and without UV treatment analyzed by ICP-MS. Error bars represent standard deviation with $\mathrm{n}=9$.

The difference between leaching of Zn for samples with UV treatment compared to that without were not significant nor consistent. TEM images in Table 4-1 and Table 4-2 also indicated no additional transformations due to UV treatment. The sweat solution, which is acidic, caused the most amount of $\mathrm{Zn}$ leaching from socks, $\mathrm{t}$-shirt, and powder. Due to sunscreen being in a liquid form, all solutions showed a high amount of $\mathrm{Zn}$ leaching. The effects of an acidic solution on the leaching $\mathrm{Zn}$ is consistent with dissolution observed in TEM images (Table 4-1 and Table 4-2). 
Once these nanoparticles have leached out of commercial products, they will make their way into the sewage system, where they would mix with raw wastewater before being going through the various processes of the wastewater treatment plant. To simulate this phase of the life cycle of nanoparticles, the solutions of leached nanoparticles obtained after day-to-day use (Table 4-1) were mixed with raw wastewater at a 1:1 ratio and incubated for 24 hours (Table 4-3). 
Table 4-3: TEM images of nanoparticles extracted from commercial products that had been incubated with raw wastewater for 24-hours after being subjected to day-to-day use conditions. Please refer to supporting information (Figure $\mathbf{S}$ 4-13, Figure $\mathbf{S}$ 4-14, Figure S 4-15, and Figure S 4-16) for EDS spectra. Arrows indicate agglomerated particles due to dissolution.

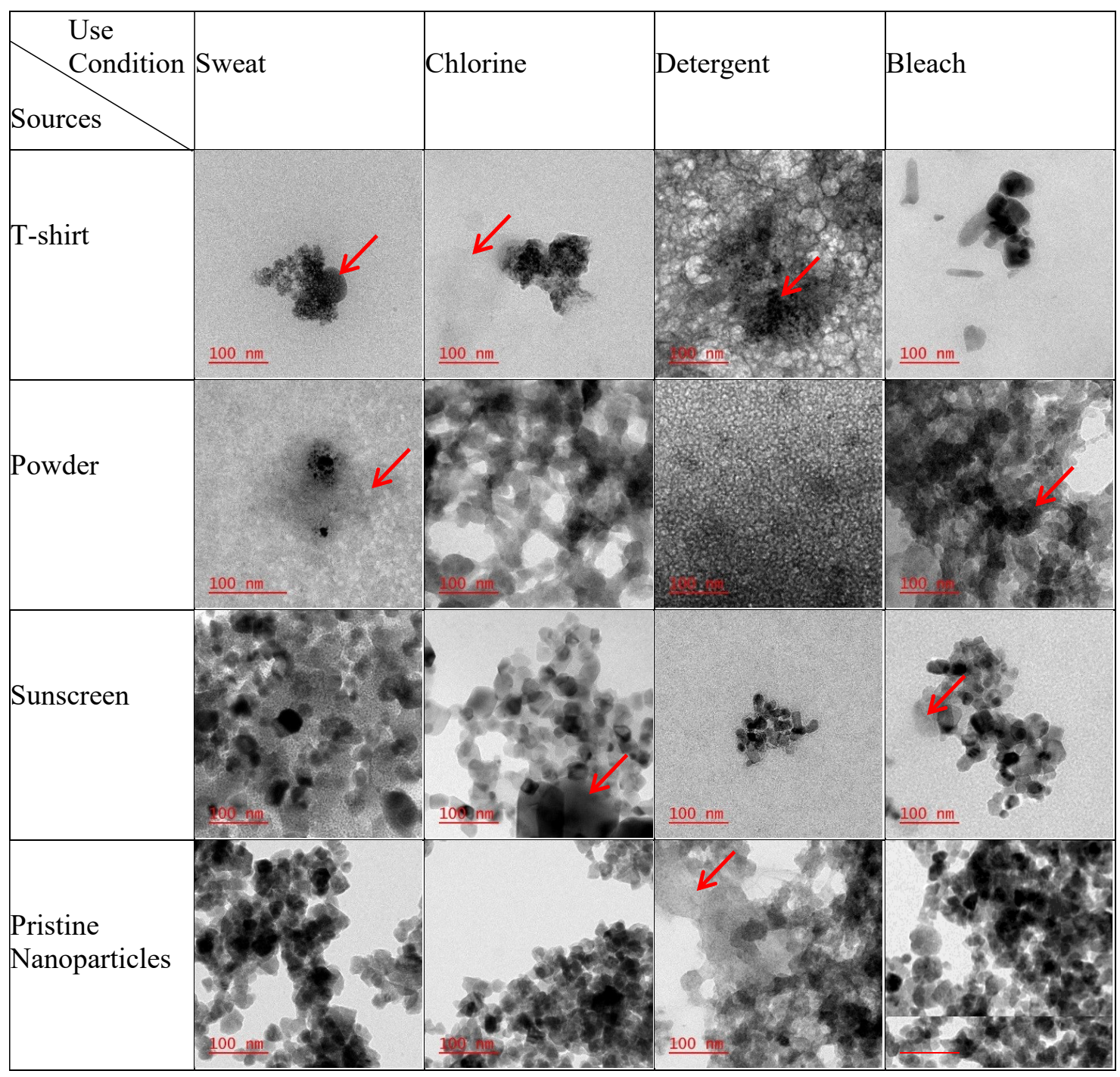

Agglomeration and dissolution of the nanoparticles were observed after treatment

with wastewater due to the low $\mathrm{pH}$ and the various contaminants present (Table 4-3).

The agglomeration was shown by the larger particles that are forming and the dissolution of the nanoparticles are observed as a cloudy haze around the nanoparticles. EDS was performed on the cloudy haze and $\mathrm{Zn}$ was detected, which suggests the dissolution of 
$\mathrm{ZnO}$ NPs. Moreover, the association of $\mathrm{S}$ and $\mathrm{Cl}$ with $\mathrm{Zn}$ was consistently observed in the EDS analysis (Figure S 4-13, Figure S 4-14, Figure S 4-15, and Figure S 4-16) throughout all the samples.

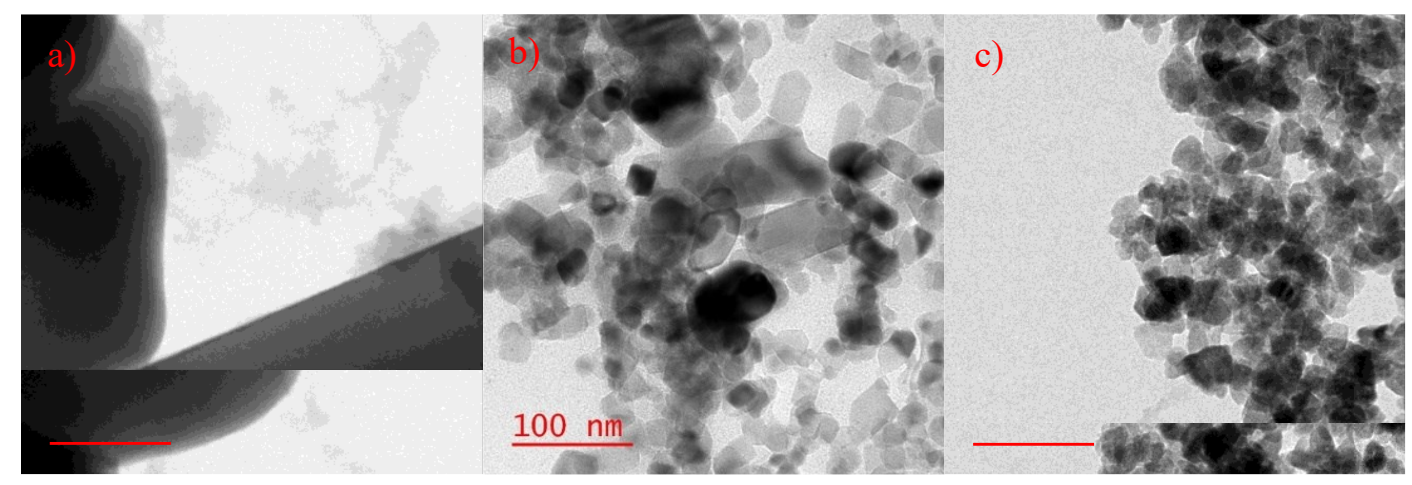

Figure 4-6: TEM images of a) powder, b) sunscreen, and c) pristine nanoparticles incubated in raw wastewater for 24-hours to mimic wastewater collection. Please refer to supporting information (Figure S 4-12) for EDS peaks.

Since wastewater had shown to impart the greatest degree of transformation, the powder, sunscreen, and pristine nanoparticles were directly introduced to wastewater without any prior weathering (Figure 4-6). The physical and chemical morphology of $\mathrm{ZnO}$ nanoparticles directly incubated in wastewater was similar to the nanoparticles that were weathered through day-to-day use before treating with wastewater.

Through this study, it can be concluded that ZnO NPs do show extensive physical and chemical transformation during the day-to-day use and wastewater treatment process. Dissolution of $\mathrm{ZnO}$ as $\mathrm{Zn}^{2+}$ and the formation of $\mathrm{ZnCl}_{2}$ and $\mathrm{ZnS}$ are the primary outcome of the weathering. The toxicity of the transformed ZnO NPs would not be diminished as the particle solubility is one of the factors that contribute to the cytotoxicity of $\mathrm{ZnO}^{42,43}$. $\mathrm{ZnO}$ in the nanoparticle form will still be present in wastewater as the complete dissolution was not observed through the parameters in this study. The final weathered 
nanoparticles must be used to assess the environmental effects of $\mathrm{ZnO}$ accurately. However, following the steps of the full life cycle of the nanoparticles can be lengthy. This study has shown that wastewater incubation had the most pronounced conformational and morphological transformation on ZnO NPs. Therefore, pristine nanoparticle exposure to a 24-hour wastewater incubation alone should be sufficient in obtaining samples for more accurate environmental studies. To further this study, the transformation of pristine and weathered $\mathrm{ZnO}$ NPs during the wastewater and biosolids treatment process will be pursued.

\subsection{Acknowledgment}

MCD acknowledges Environment and Climate Change Canada for a Grants and Contributions Agreement to support this research. This work would not be possible without the technical help of Jianqun Wang, the TEM and SEM facility manager in obtaining TEM images and troubleshooting the instrument. 


\subsection{References}

1. The Project on Emerging Nanotechnologies. (2013). Available at: http://www.nanotechproject.org/cpi/about/analysis/. (Accessed: 1st April 2015)

2. Wiegand, C., Hipler, U.-C., Boldt, S., Strehle, J. \& Wollina, U. Skin-protective effects of a zinc oxide-functionalized textile and its relevance for atopic dermatitis. Clin. Cosmet. Investig. Dermatol. 6, 115-21 (2013).

3. Jones, N., Ray, B., Ranjit, K. T. \& Manna, A. C. Antibacterial activity of ZnO nanoparticle suspensions on a broad spectrum of microorganisms. FEMS Microbiol. Lett. 279, 71-76 (2008).

4. Gottschalk, F., Sonderer, T., Scholz, R. W. \& Nowack, B. Modeled Environmental Concentrations of Engineered Nanomaterials ( $\mathrm{TiO}_{2}, \mathrm{ZnO}, \mathrm{Ag}, \mathrm{CNT}$, Fullerenes) for Different Regions. Environ. Sci. Technol. 43, 9216-9222 (2009).

5. Meulenkamp, E. A. Synthesis and Growth of ZnO Nanoparticles. J. Phys. Chem. B 102, 5566-5572 (1998).

6. Wang, Z. et al. Low-temperature synthesis of $\mathrm{ZnO}$ nanoparticles by solid-state pyrolytic reaction. Nanotechnology 14, 11 (2003).

7. Hong, R. Y. et al. Synthesis, surface modification and photocatalytic property of ZnO nanoparticles. Powder Technol. 189, 426-432 (2009).

8. Min, Y.-S. et al. $\mathrm{ZnO}$ nanoparticle growth on single-walled carbon nanotubes by atomic layer deposition and a consequent lifetime elongation of nanotube field emission. Appl. Phys. Lett. 90, (2007).

9. Vrinceanu, N. et al. Zinc Oxide - Linen Fibrous Composites: Morphological, Structural, Chemical, Humidity Adsorptive and Thermal Barrier Attributes. in Modern Surface Engineering Treatments 21-44 (2013).

10. Brayner, R. et al. Toxicological impact studies based on Escherichia coli bacteria in ultrafine $\mathrm{ZnO}$ nanoparticles colloidal medium. Nano Lett. 6, 866-870 (2006).

11. Bai, W. et al. Toxicity of zinc oxide nanoparticles to zebrafish embryo: a physicochemical study of toxicity mechanism. J. Nanoparticle Res. 12, 1645-1654 (2009).

12. Xia, T. et al. Comparison of the mechanism of toxicity of zinc oxide and cerium oxide nanoparticles based on dissolution and oxidative stress properties. ACS Nano 2, 2121-2134 (2008).

13. Premanathan, M., Karthikeyan, K., Jeyasubramanian, K. \& Manivannan, G. Selective toxicity of $\mathrm{ZnO}$ nanoparticles toward Gram-positive bacteria and cancer 
cells by apoptosis through lipid peroxidation. Nanomedicine Nanotechnology, Biol. Med. 7, 184-192 (2011).

14. Ma, H., Williams, P. L. \& Diamond, S. A. Ecotoxicity of manufactured ZnO nanoparticles - A review. Environ. Pollut. 172, 76-85 (2013).

15. Xia, T. et al. Comparison of the mechanism of toxicity of zinc oxide and cerium oxide nanoparticles based on dissolution and oxidative stress properties. ACS Nano 2, 2121-2134 (2008).

16. Feris, K. et al. Electrostatic Interactions Affect Nanoparticle-Mediated Toxicity to Gram-Negative Bacterium Pseudomonas aeruginosa PAO1. Langmuir 26, 44294436 (2010).

17. Hotze, E. M., Phenrat, T. \& Lowry, G. V. Nanoparticle aggregation: challenges to understanding transport and reactivity in the environment. J. Environ. Qual. 39, 1909-1924 (2010).

18. Bian, S.-W., Mudunkotuwa, I. A., Rupasinghe, T. \& Grassian, V. H. Aggregation and Dissolution of $4 \mathrm{~nm} \mathrm{ZnO}$ Nanoparticles in Aqueous Environments: Influence of pH, Ionic Strength, Size, and Adsorption of Humic Acid. Langmuir 27, 60596068 (2011).

19. Dale, A. L., Lowry, G. V. \& Casman, E. A. Stream Dynamics and Chemical Transformations Control the Environmental Fate of Silver and Zinc Oxide Nanoparticles in a Watershed-Scale Model. Environ. Sci. Technol. 49, 7285-7293 (2015).

20. Almeida, L. \& Ramos, D. Health and safety concerns of textiles with nanomaterials. IOP Conf. Ser. Mater. Sci. Eng. 254, 102002 (2017).

21. Zhao, H. \& Li, R. K. Y. A study on the photo-degradation of zinc oxide (ZnO) filled polypropylene nanocomposites. Polymer (Guildf). 47, 3207-3217 (2006).

22. Pourrahimi, A. M. et al. Water-based synthesis and cleaning methods for high purity $\mathrm{ZnO}$ nanoparticles - comparing acetate, chloride, sulphate and nitrate zinc salt precursors. RSC Adv. 4, 35568-35577 (2014).

23. Kulthong, K., Srisung, S., Boonpavanitchakul, K., Kangwansupamonkon, W. \& Maniratanachote, R. Determination of silver nanoparticle release from antibacterial fabrics into artificial sweat. Part. Fibre Toxicol. 7, 8 (2010).

24. Barker, P. J. \& Branch, A. The interaction of modern sunscreen formulations with surface coatings. Prog. Org. Coatings 62, 313-320 (2008).

25. Benn, T. M. \& Westerhoff, P. Nanoparticle silver released into water from commercially available sock fabrics. Environ. Sci. Technol. 42, 4133-9 (2008). 
26. Salter, C., Langhus, D. L. \& Kauffman, G. B. Chemistry for Everyone The Chemistry of Swimming Pool Maintenance W Products of Chemistry edited by. Journal of Chemical Education 84, (2007).

27. Mrówka, M. et al. Study of selected physical, chemical and biological properties of selected materials intended for contact with human body. Polish J. Chem. Technol. 21, 1-8 (2019).

28. Song, W. et al. Role of the dissolved zinc ion and reactive oxygen species in cytotoxicity of ZnO nanoparticles. Toxicol. Lett. 199, 389-397 (2010).

29. Pasquet, J. et al. The contribution of zinc ions to the antimicrobial activity of zinc oxide. Colloids Surfaces A Physicochem. Eng. Asp. 457, 263-274 (2014).

30. Meulenkamp, E. A. Size Dependence of the Dissolution of ZnO Nanoparticles. J. Phys. Chem. B 102, 7764-7769 (1998).

31. Smijs, T. G. \& Pavel, S. Titanium dioxide and zinc oxide nanoparticles in sunscreens: focus on their safety and effectiveness. Nanotechnol. Sci. Appl. 4, 95$112(2011)$.

32. Eastern Research Group. State of the Science Literature Review : Nano Titanium Dioxide Environmental Matters. Epa (2010).

33. Wesolowski, D. J., Bénézeth, P. \& Palmer, D. A. ZnO Solubility and Zn2 Complexation by Chloride and Sulfate in Acidic Solutions to $290^{\circ} \mathrm{C}$ with In-Situ pH Measurement. Geochim. Cosmochim. Acta 62, 971-984 (1998).

34. Ziemniak, S. E., Jones, M. E. \& Combs, K. E. S. Solubility behavior of titanium(IV) oxide in alkaline media at elevated temperatures. J. Solution Chem. 22, 601-623 (1993).

35. Vigneshwaran, N., Kathe, A. ., Varadarajan, P. ., Nachane, R. . \& Balasubramanya, R. . Functional Finishing of Cotton Fabrics Using Silver Nanoparticles. J. Nanosci. Nanotechnol. 7, 1893-1897 (2007).

36. Khosravian, S., Montazer, M., Malek, R. M. A. \& Harifi, T. In situ synthesis of nano $\mathrm{ZnO}$ on starch sized cotton introducing nano photo active fabric optimized with response surface methodology. Carbohydr. Polym. 132, 126-133 (2015).

37. Mohd Omar, F., Abdul Aziz, H. \& Stoll, S. Aggregation and disaggregation of $\mathrm{ZnO}$ nanoparticles: Influence of $\mathrm{pH}$ and adsorption of Suwannee River humic acid. Sci. Total Environ. 468-469, 195-201 (2014).

38. Wang, L. \& Muhammed, M. Synthesis of zinc oxide nanoparticles with controlled morphology. J. Mater. Chem. 9, 2871-2878 (1999).

39. Lombi, E. et al. Fate of Zinc Oxide Nanoparticles during Anaerobic Digestion of 
Wastewater and Post-Treatment Processing of Sewage Sludge. Environ. Sci. Technol. 46, 9089-9096 (2012).

40. Sirelkhatim, A. et al. Review on Zinc Oxide Nanoparticles: Antibacterial Activity and Toxicity Mechanism. Nano-Micro Lett. 7, 219-242 (2015).

41. Schwertfeger, D. M., Velicogna, J. R., Jesmer, A. H., Scroggins, R. P. \& Princz, J. I. Single Particle-Inductively Coupled Plasma Mass Spectroscopy Analysis of Metallic Nanoparticles in Environmental Samples with Large Dissolved Analyte Fractions. Anal. Chem. 88, 9908-9914 (2016).

42. Franklin, N. M. et al. Comparative Toxicity of Nanoparticulate $\mathrm{ZnO}$, Bulk ZnO, and $\mathrm{ZnCl}_{2}$ to a Freshwater Microalga (Pseudokirchneriella subcapitata): The Importance of Particle Solubility. Environ. Sci. Technol. 41, 8484-8490 (2007).

43. Brunner, T. J. et al. In Vitro Cytotoxicity of Oxide Nanoparticles: Comparison to Asbestos, Silica, and the Effect of Particle Solubility †. Environ. Sci. Technol. 40, 4374-4381 (2006). 


\subsection{Supporting Information}

Table S4-1: The test results for the content raw wastewater

\begin{tabular}{|c|c|c|}
\hline Test & Units & Sample \\
\hline $\begin{array}{c}\text { Chemical Oxygen Demand, } \\
\text { COD }\end{array}$ & $\mathrm{mg} / \mathrm{L}$ & 407 \\
\hline Total Nitrogen, $\mathrm{mg} / \mathrm{L} \mathrm{N}^{-1}$ & 31 \\
\hline Total Phosphorus, $\mathrm{P}$ & $\mathrm{mg} / \mathrm{L} \mathrm{PO}_{4}{ }^{3-}$ & 2.2 \\
\hline Total Sulfate, $\mathrm{S}$ & $\mathrm{mg} / \mathrm{L} \mathrm{SO}_{4}{ }^{-}$ & 98 \\
\hline Ammonia, $\mathrm{NH}_{3}-\mathrm{N}$ & $\mathrm{mg} / \mathrm{L} \mathrm{NH}_{3}-\mathrm{N}$ & 28 \\
\hline Nitrate, NO${ }_{3}-\mathrm{N}$ & $\mathrm{mg} / \mathrm{L} \mathrm{NO}_{3}{ }^{-}-\mathrm{N}$ & 0.7 \\
\hline Turbudity & $\mathrm{NTU}$ & 123 \\
\hline Absorbance $@ 254 \mathrm{~nm}$ & - & 1.3303 \\
\hline Total Solids & $\mathrm{mg} / \mathrm{L}$ & 907 \\
\hline Total Volatile Solids & $\mathrm{mg} / \mathrm{L}$ & 326 \\
\hline
\end{tabular}



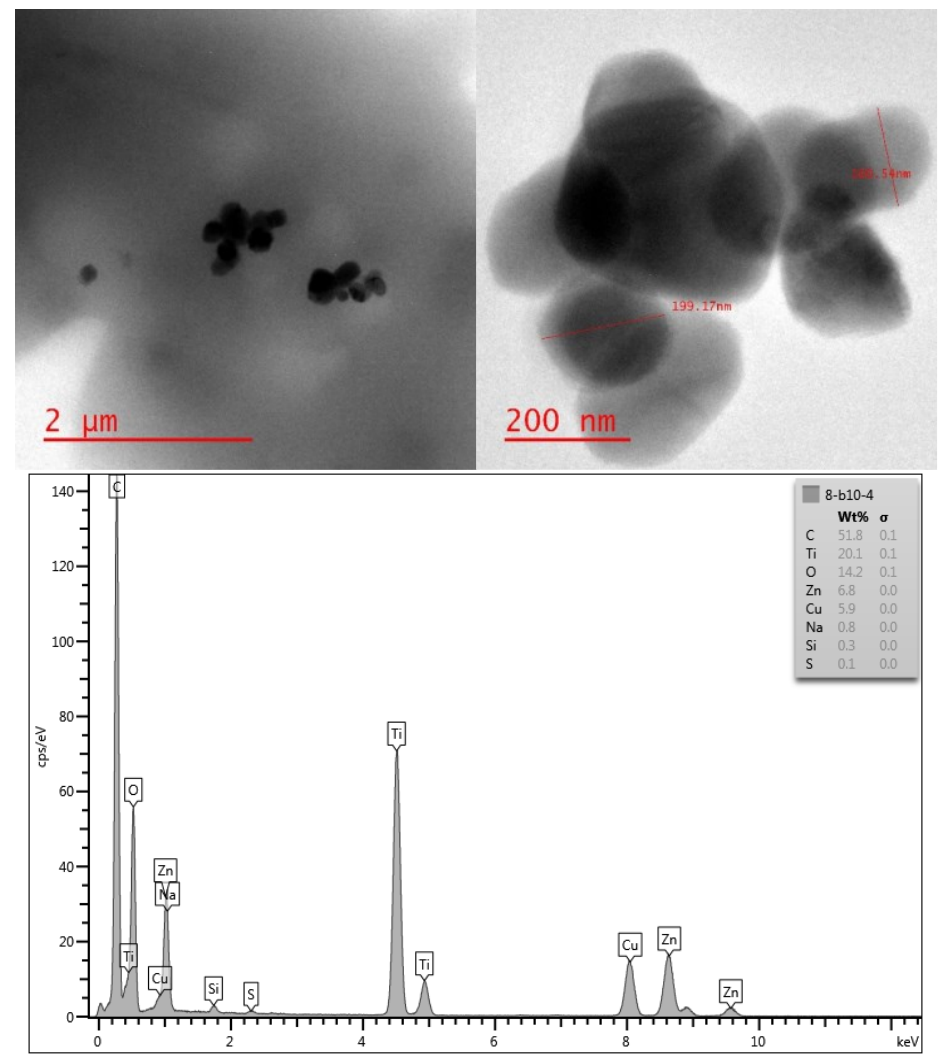

Figure S 4-1: (top) TEM images and (bottom) EDS peaks of BEHR Ultra white paint extracted through incineration. 

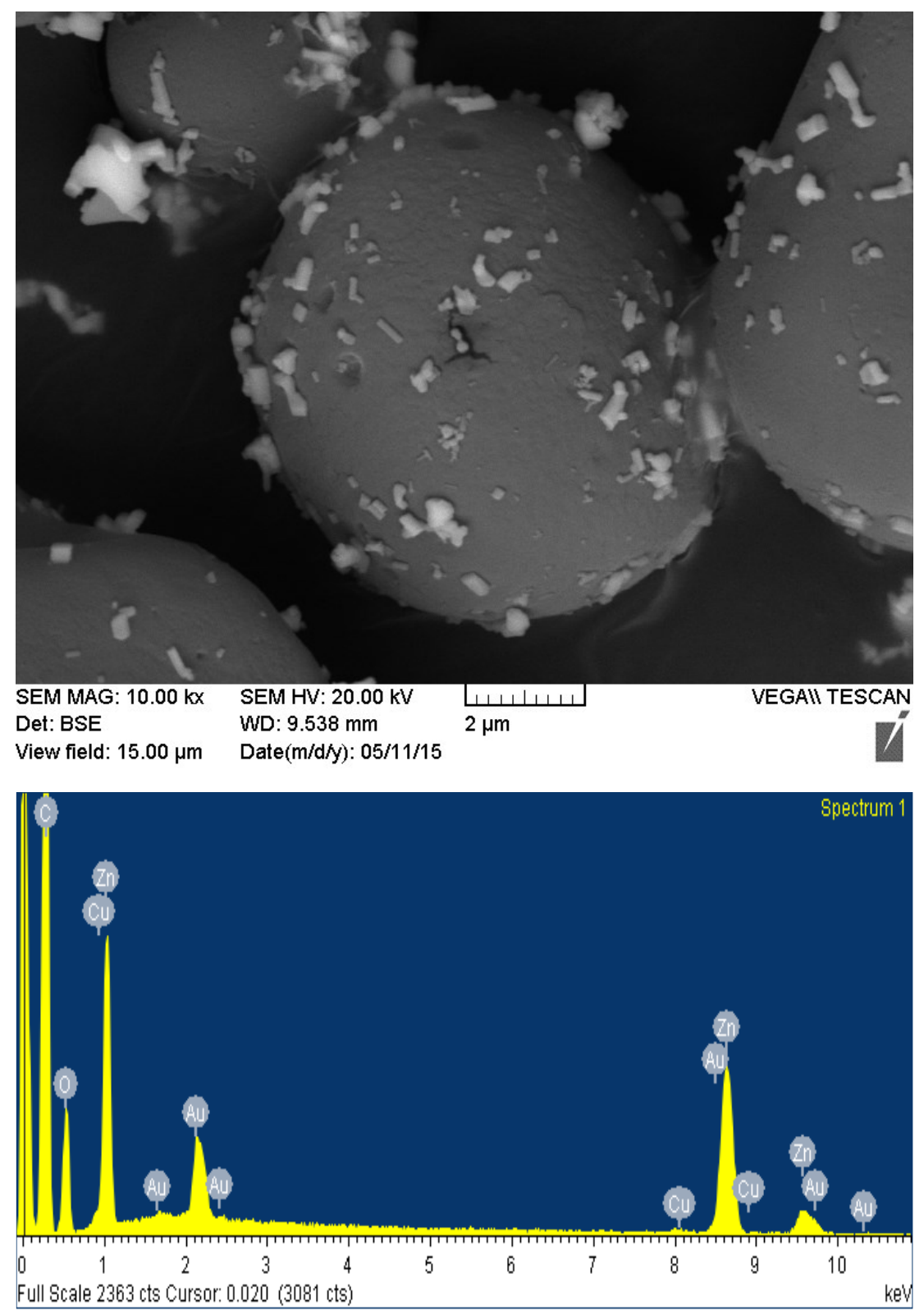

Figure S 4-2: SEM image and EDS spectrum of Johnson's baby powder showing ZnO around the silicon particles present in talc. 


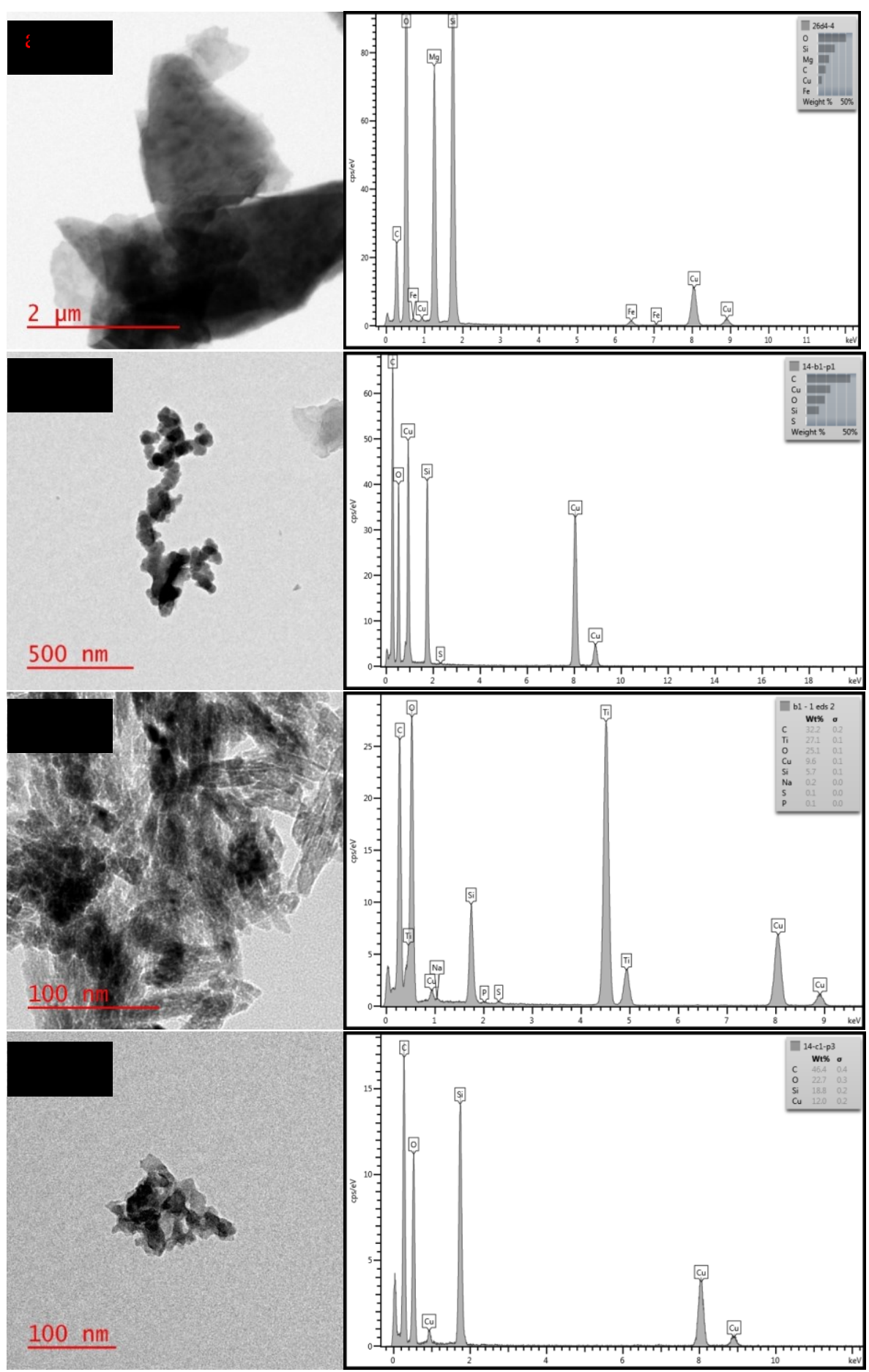

Figure S 4-3: TEM images and EDS peaks of control sources prepared by incineration: a) Johnson's baby powder, b)Aveeno Baby sunscreen lotion SPF 55, c)Garnier Ombrella Kids hypoallergenic lotion SPF 50+ and d)Neutrogena Ultra Sheer face sunscreen SPF 60 . 


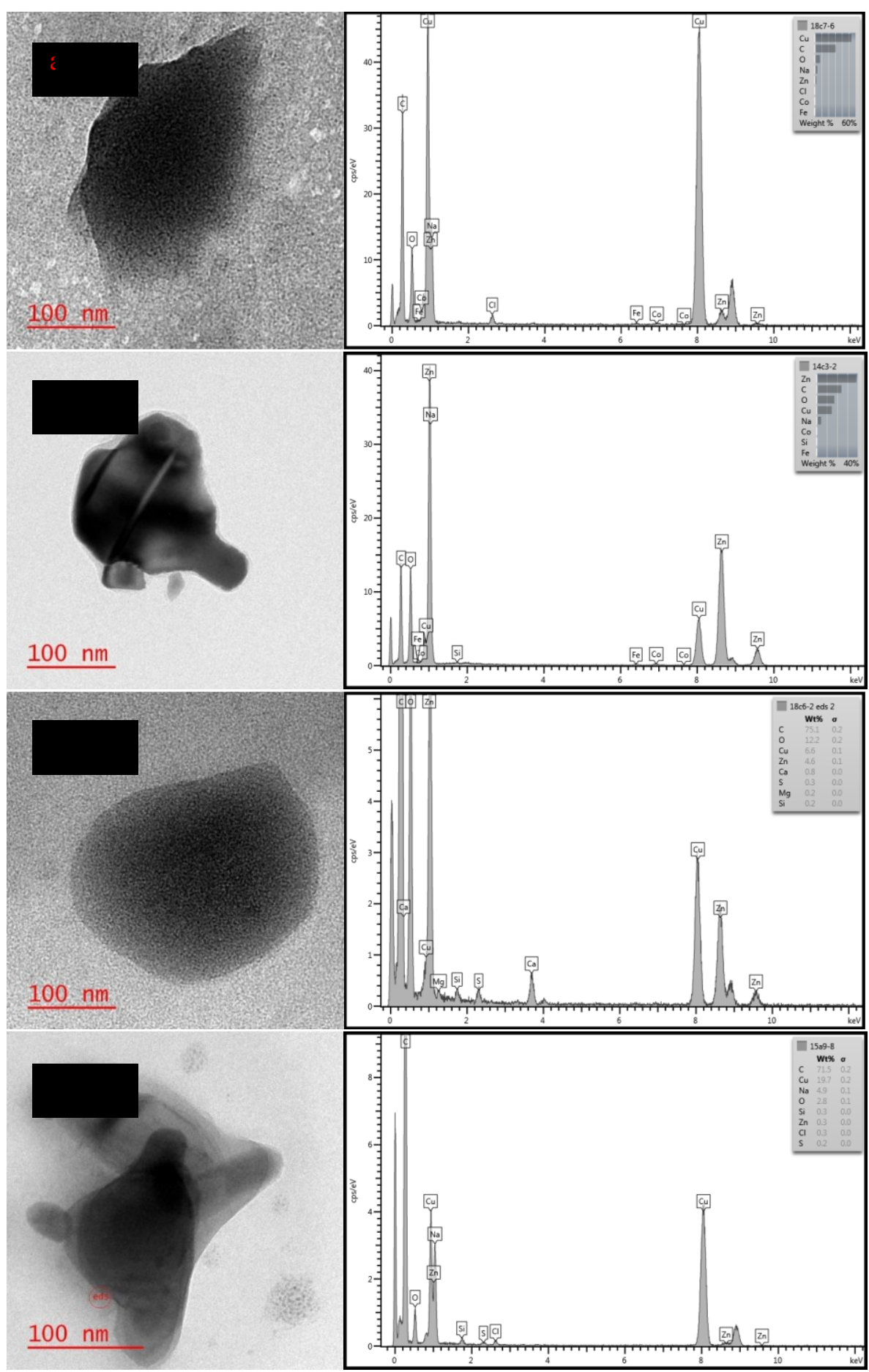

Figure S 4-4: TEM images and EDS peaks of solutions of $\mathrm{ZnO}$ from t-shirt after day-today use conditions: a)sweat, b)chlorine, c)detergent and d)bleach. 


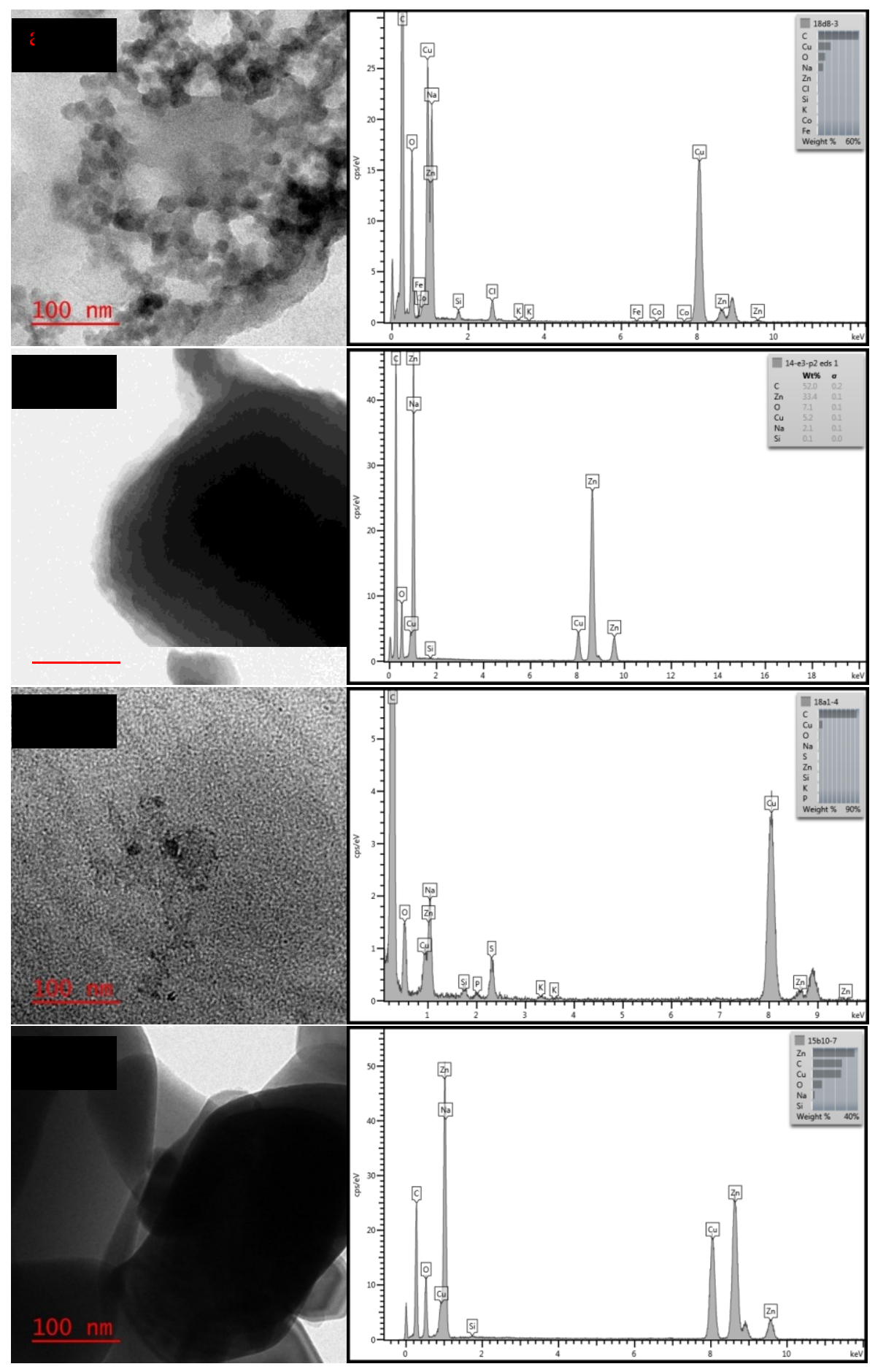

Figure S 4-5: TEM images and EDS peaks of solutions of $\mathrm{ZnO}$ from baby powder after day-to-day use conditions: a) sweat, b) chlorine, c) detergent and d) bleach. 


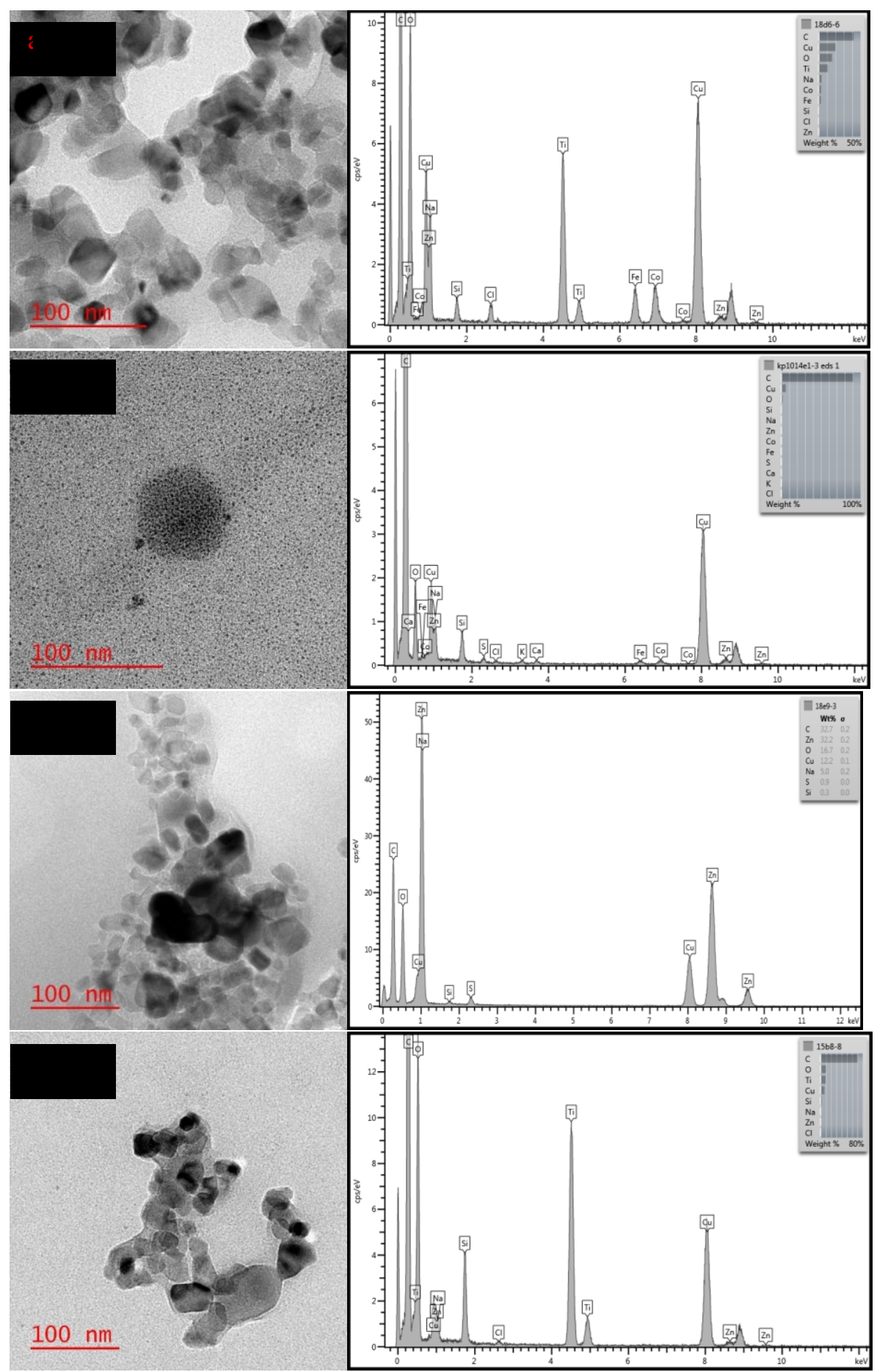

Figure S 4-6: TEM images and EDS peaks of solutions of $\mathrm{ZnO}$ from sunscreen after dayto-day use conditions: a) sweat, b) chlorine, c) detergent and d) bleach. 


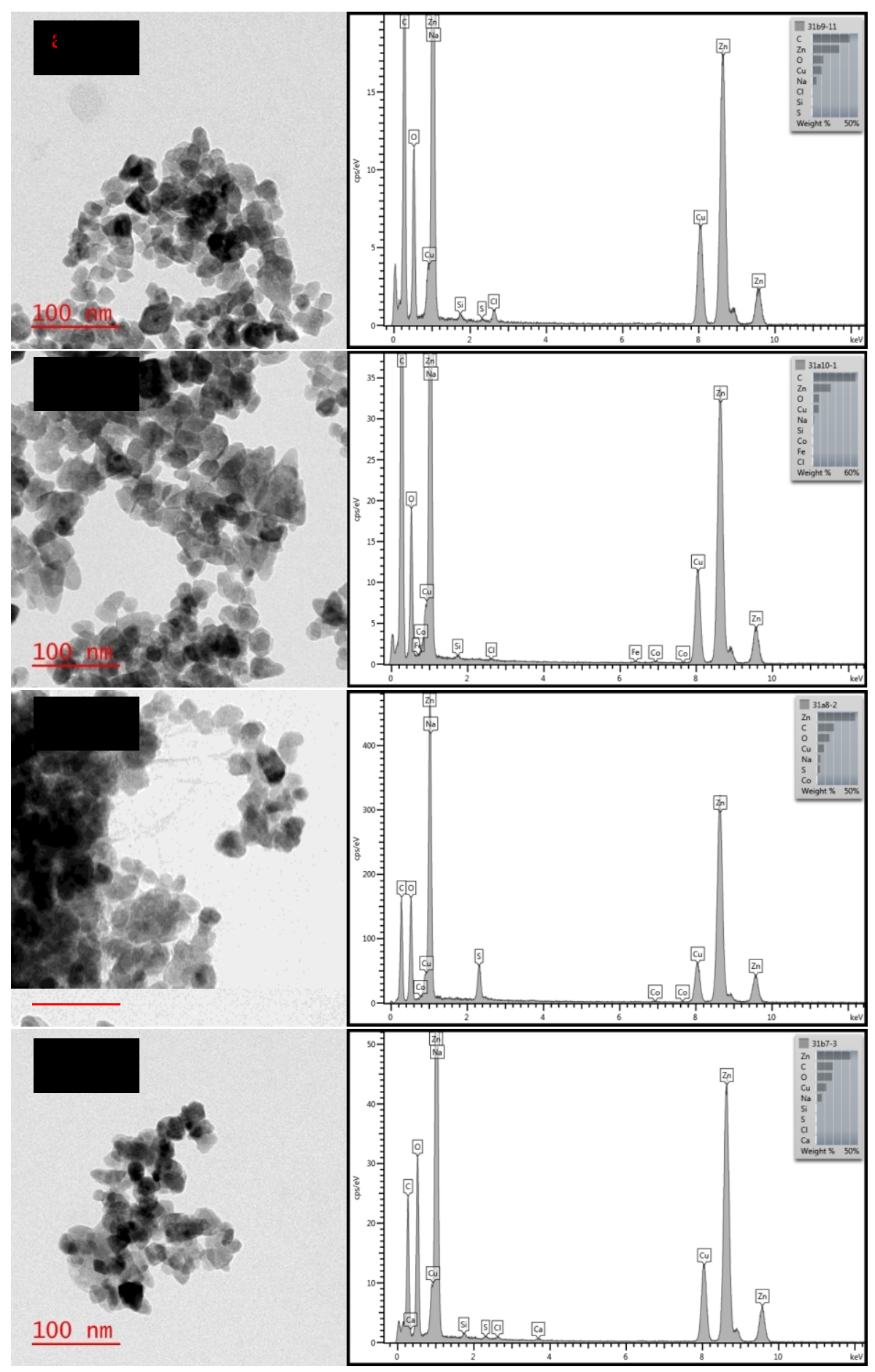

Figure S 4-7: TEM images and EDS peaks of solutions of $\mathrm{ZnO}$ from pristine nanoparticles after day-to-day use conditions: a)sweat, b)chlorine, c)detergent and d)bleach. 


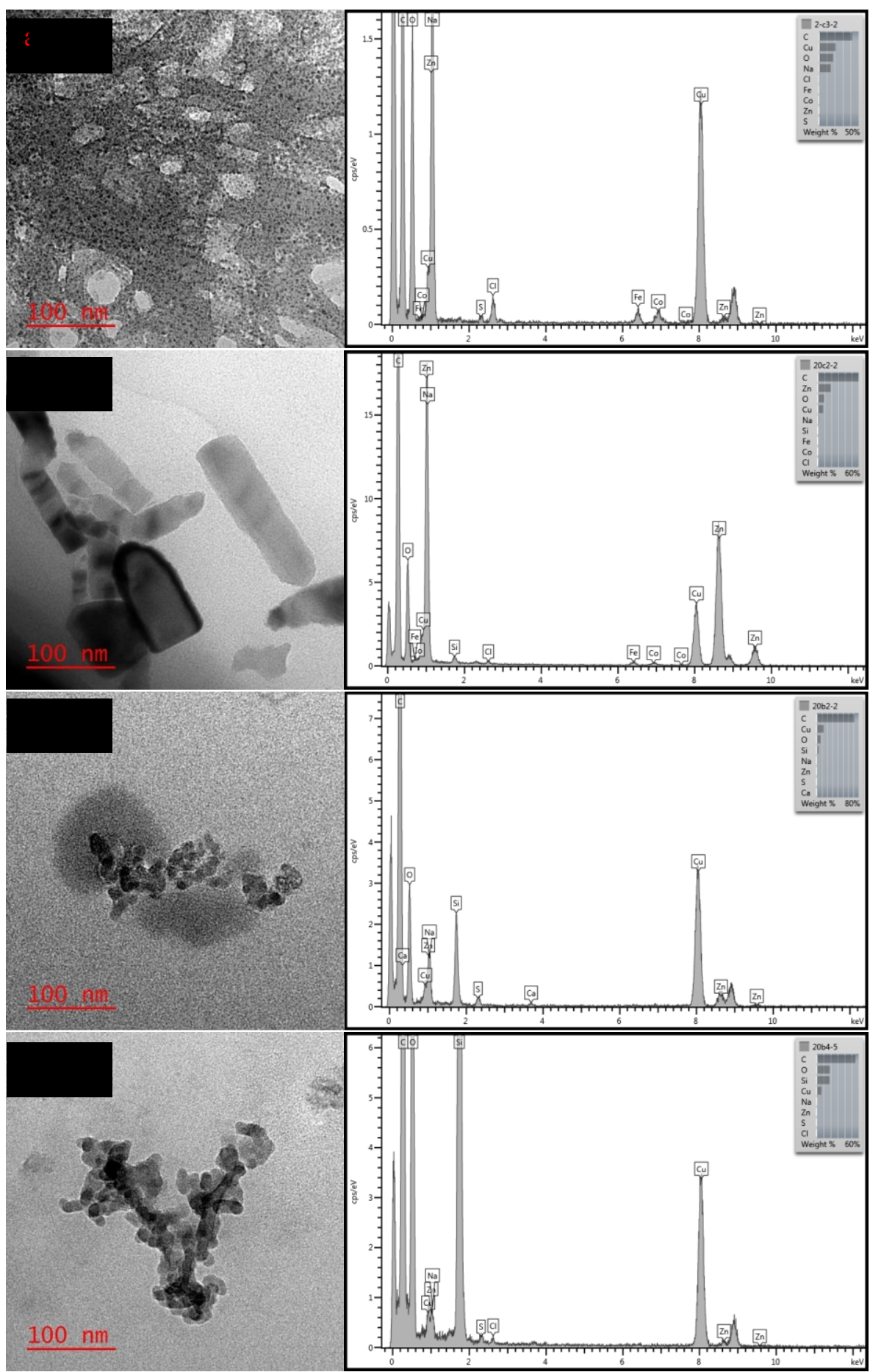

Figure S 4-8: TEM images and EDS peaks of solutions of $\mathrm{ZnO}$ from t-shirt after exposed to UV light and day-to-day use conditions: a)sweat, b)chlorine, c)detergent and d)bleach. 


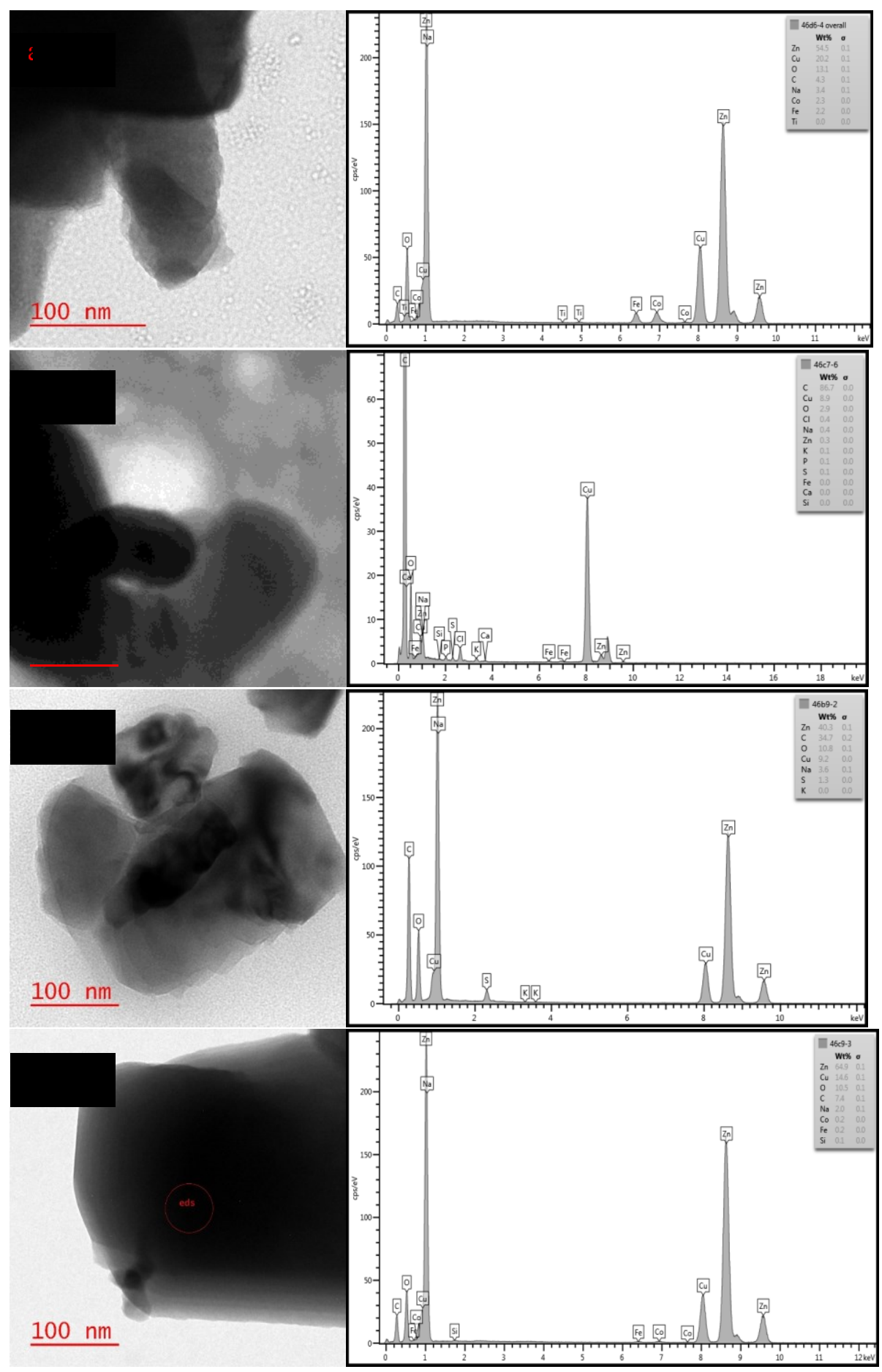

Figure S 4-9: TEM images and EDS peaks of solutions of $\mathrm{ZnO}$ from baby powder after exposed to UV light and day-to-day use conditions: a)sweat, b)chlorine, c)detergent and d)bleach. 


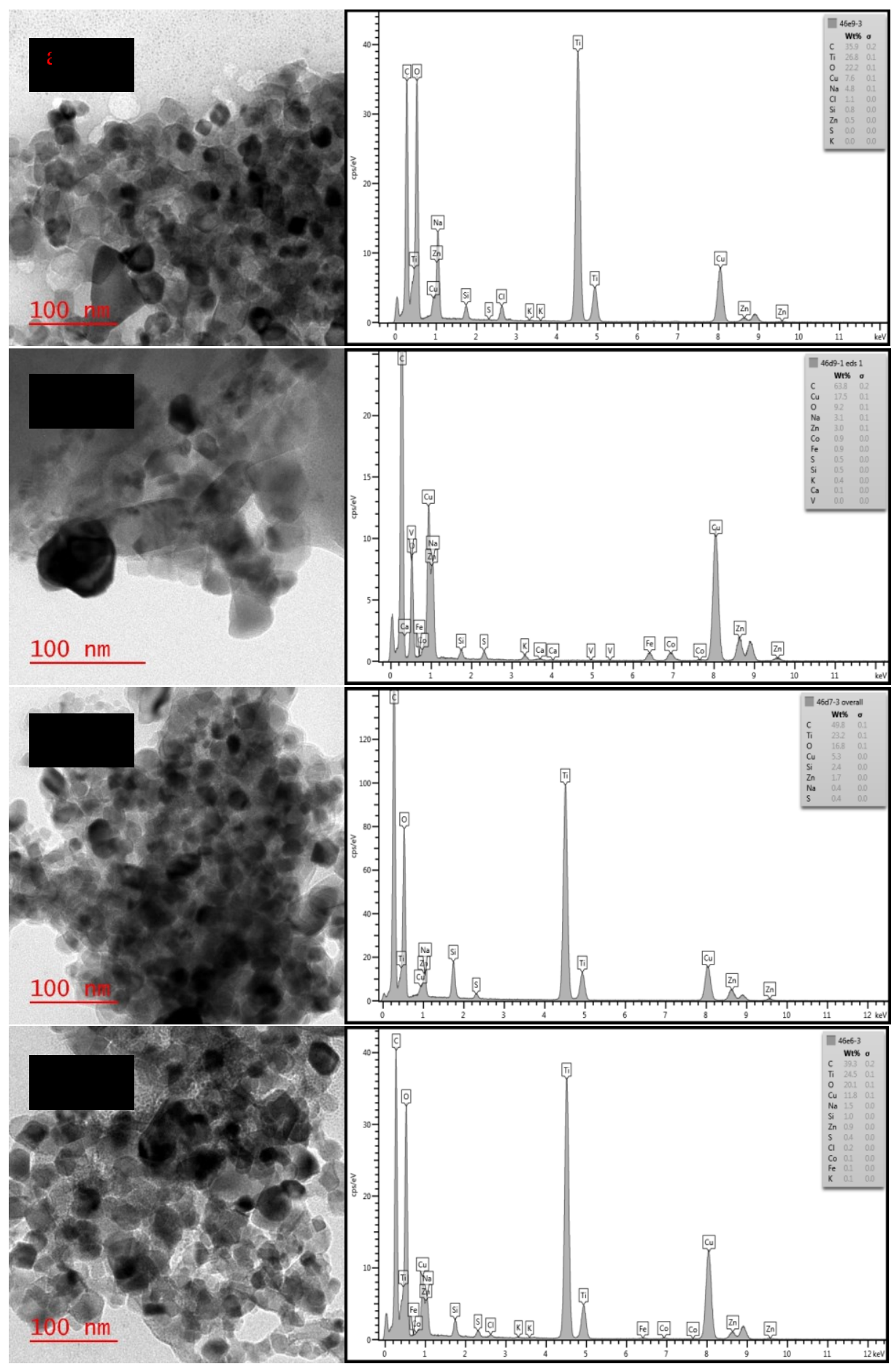

Figure S 4-10: TEM images and EDS peaks of solutions of $\mathrm{ZnO}$ from sunscreen after exposed to UV light and day-to-day use conditions: a)sweat, b)chlorine, c)detergent and d)bleach. 


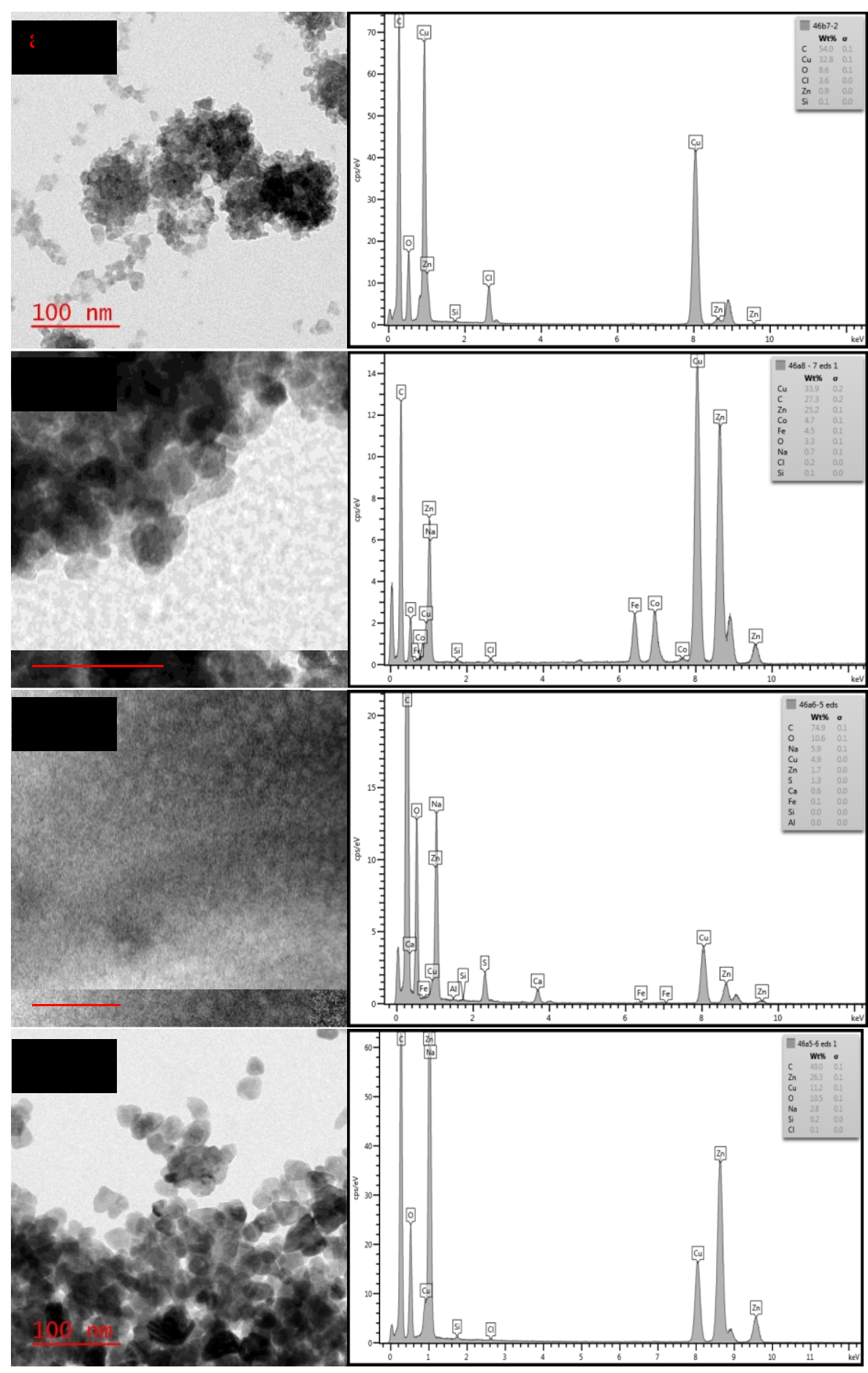

Figure S 4-11: TEM images and EDS peaks of solutions of $\mathrm{ZnO}$ from pristine nanoparticles after exposed to UV light and day-to-day use conditions: a)sweat, b)chlorine, c)detergent and d)bleach 


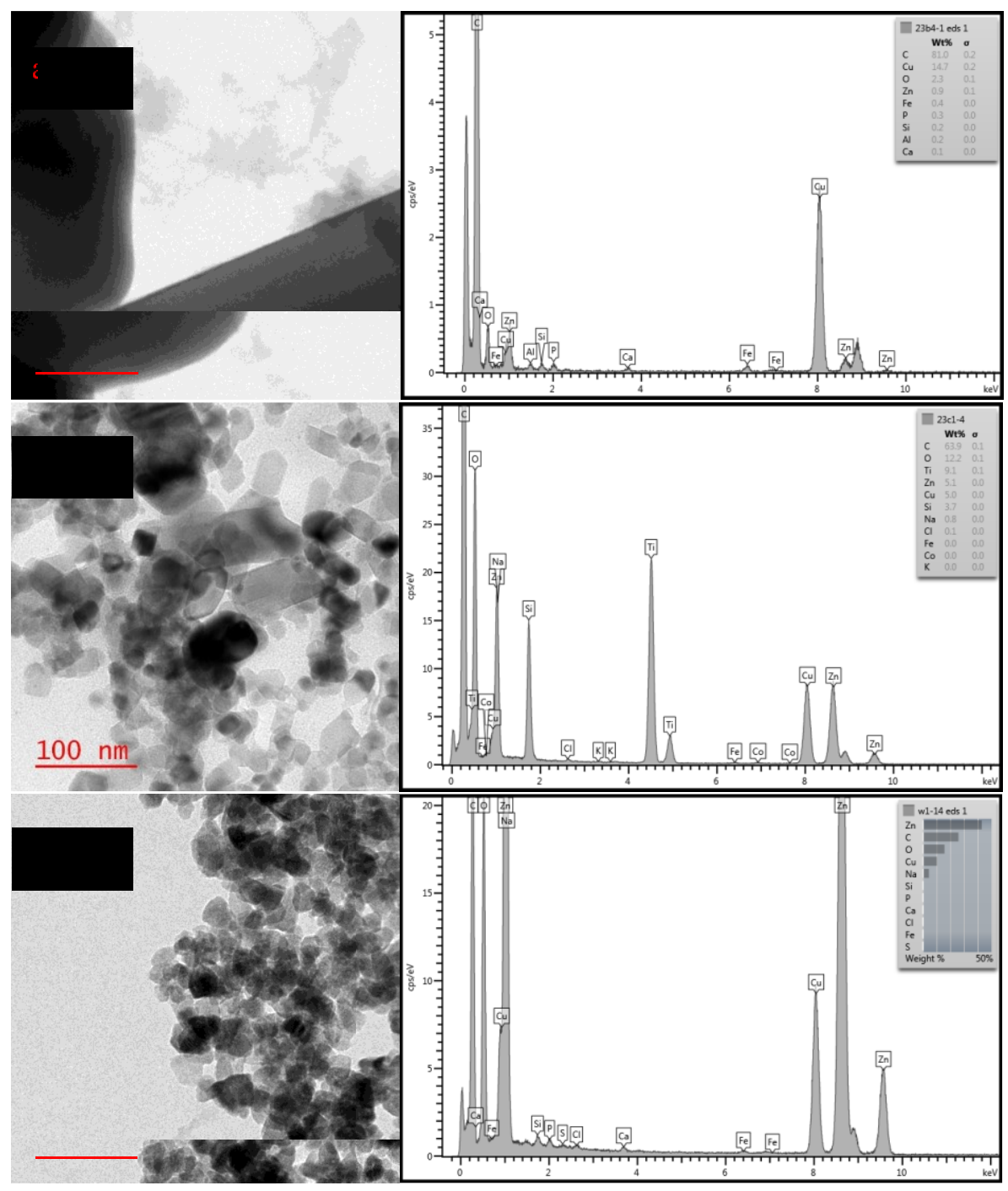

Figure S 4-12: TEM images and EDS peaks of a) baby powder, b) sunscreen and c) pristine nanoparticles in wastewater. 


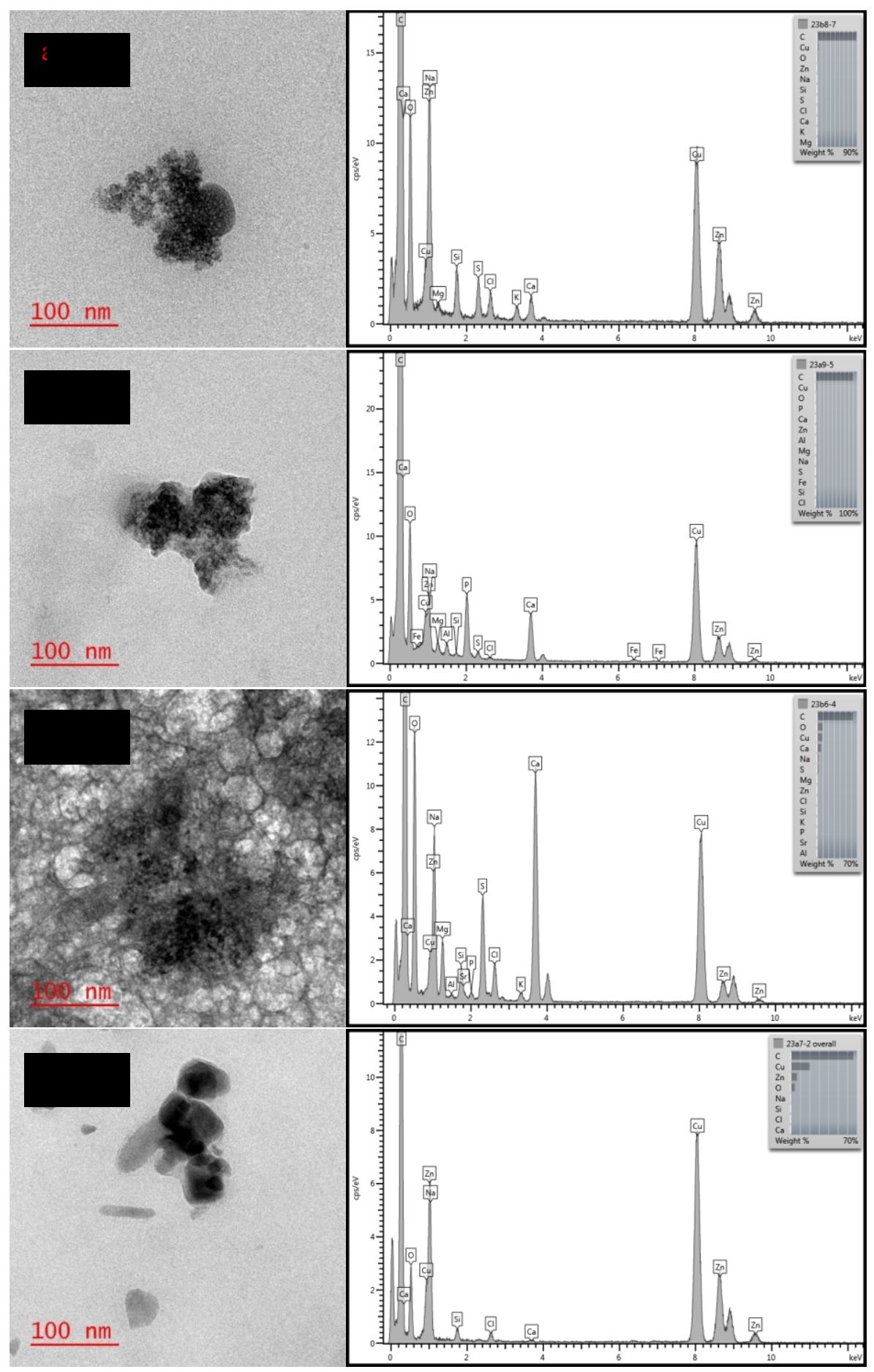

Figure S 4-13: TEM images and EDS peaks of mixture of wastewater and solutions of tshirt after day-to-day conditions are applied a) sweat, b) chlorine, c) detergent and d) bleach. 


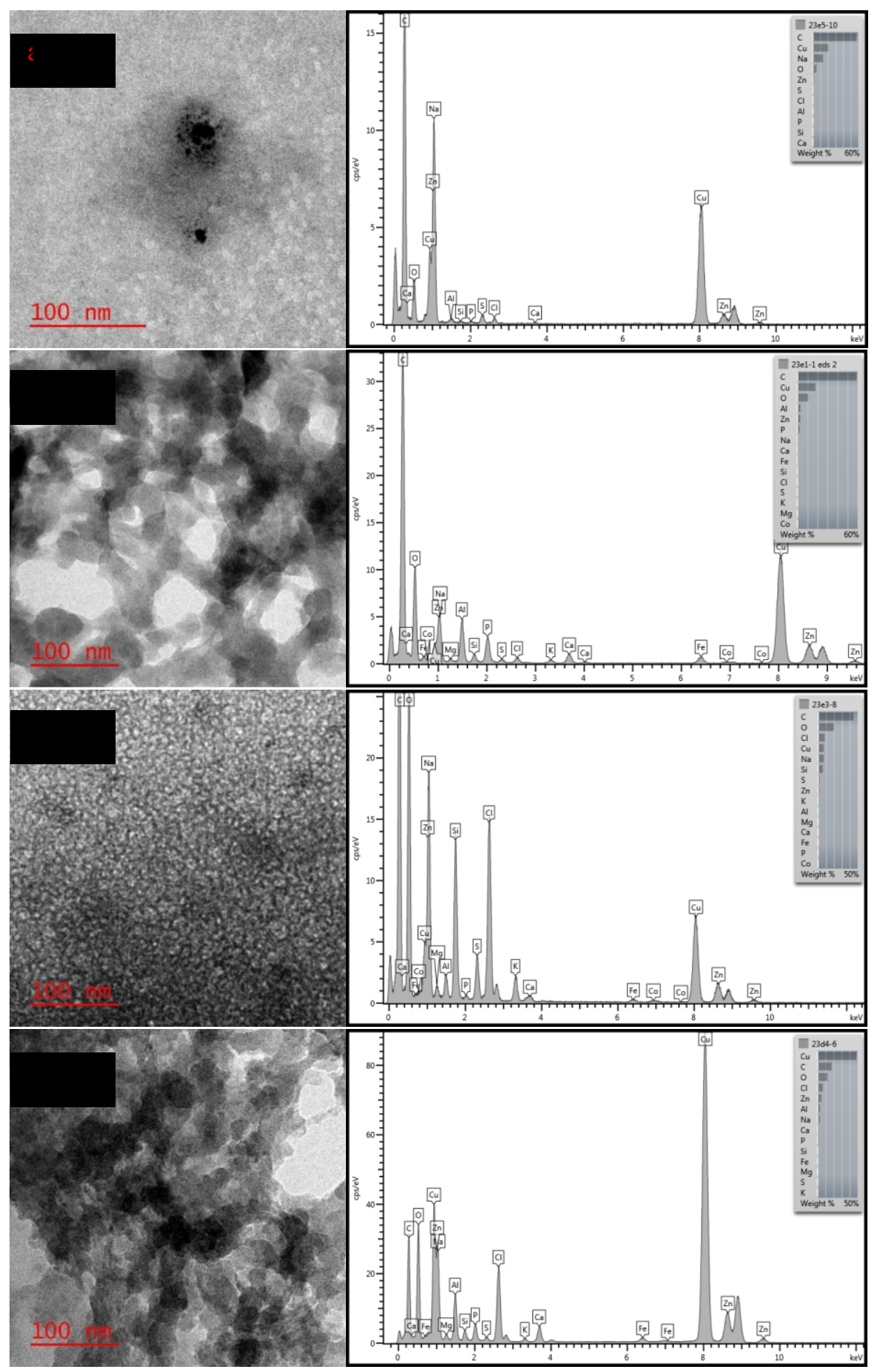

Figure S 4-14: TEM images and EDS peaks of mixture of wastewater and solutions of baby powder after day-to-day conditions are applied a) sweat, b) chlorine, c) detergent and d) bleach. 


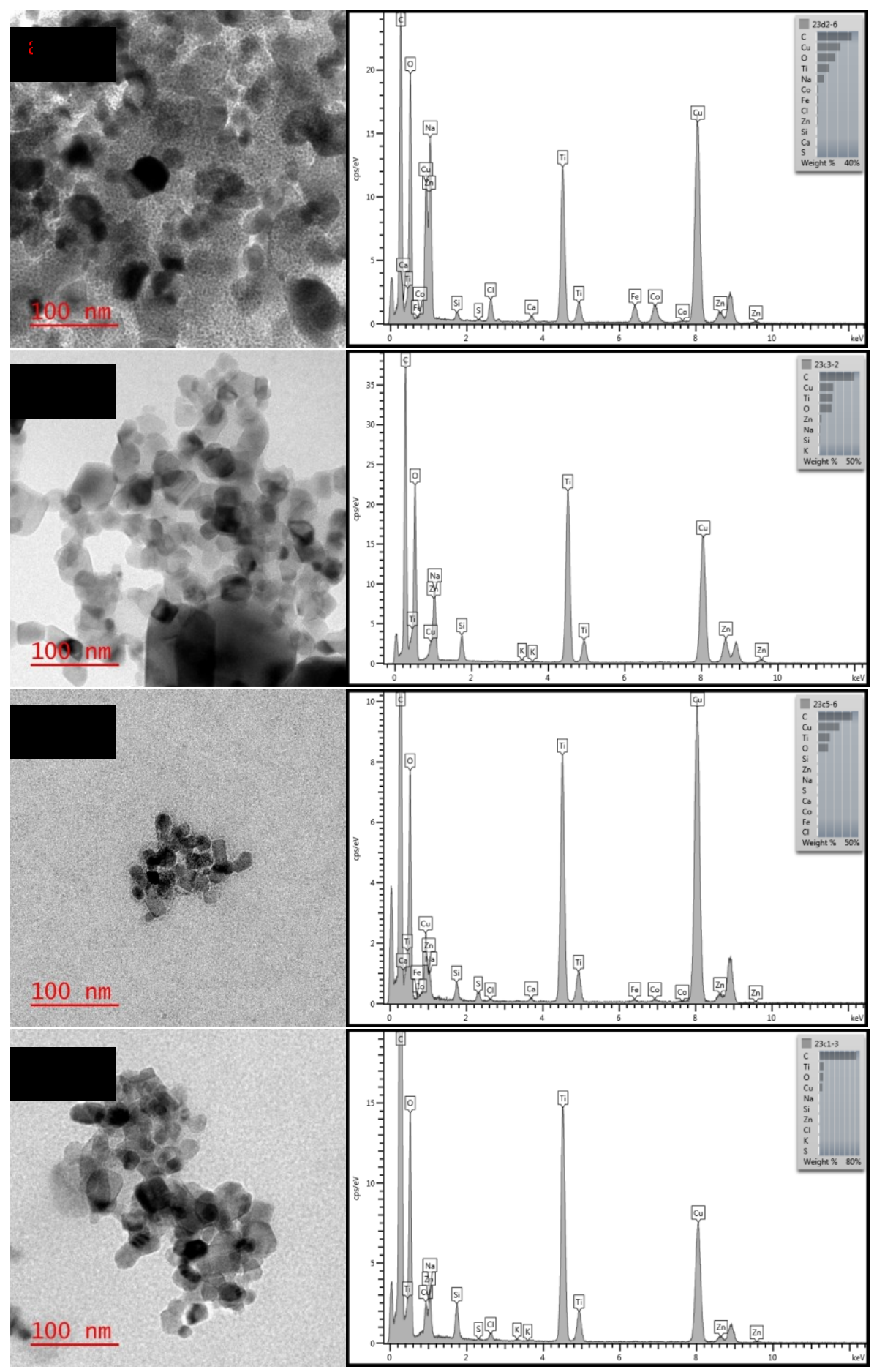

Figure S 4-15: TEM images and EDS peaks of mixture of wastewater and solutions of sunscreen after day-to-day conditions are applied a) sweat, b) chlorine, c) detergent and d) bleach. 


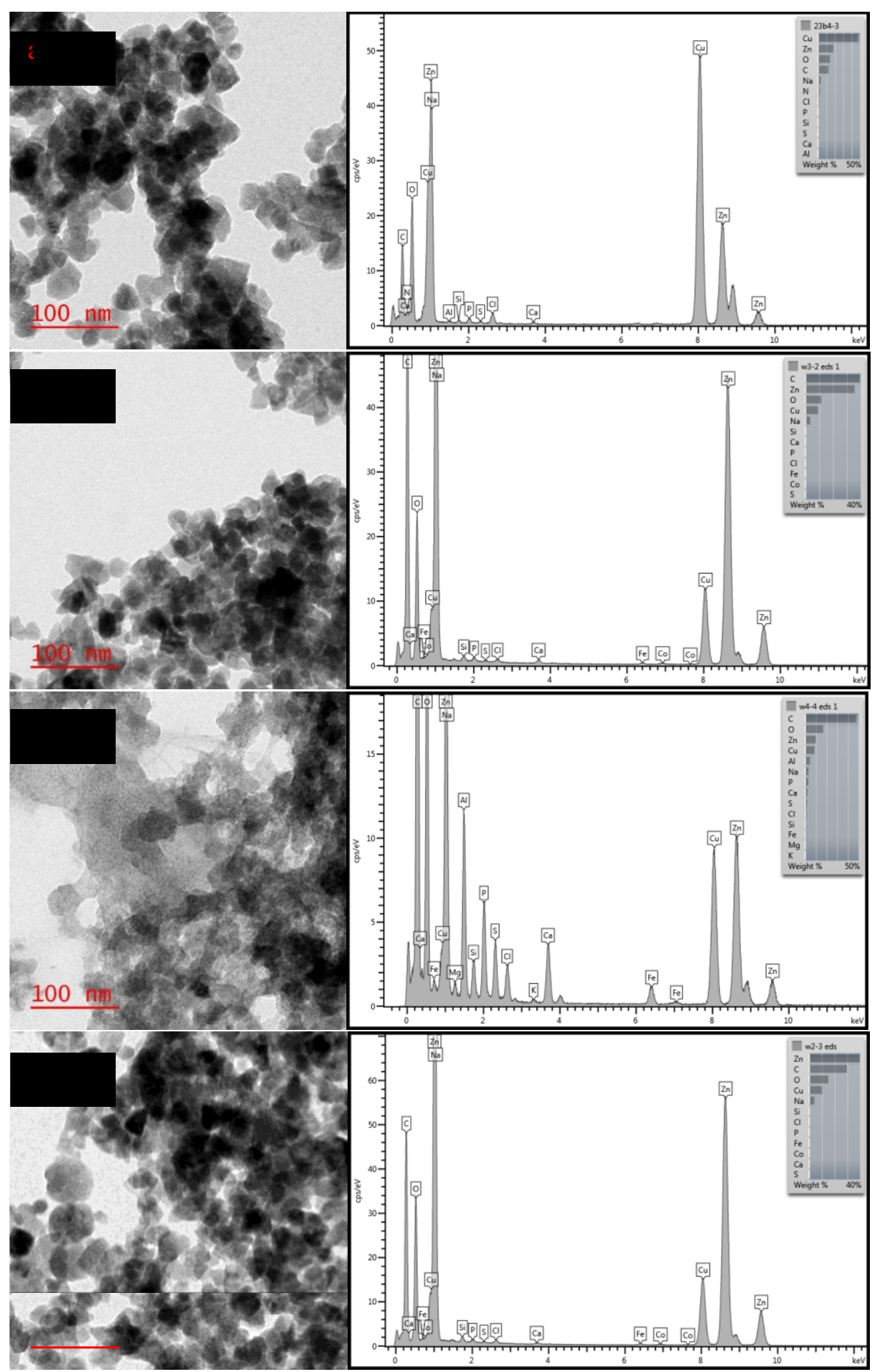

Figure S 4-16: TEM images and EDS peaks of mixture of wastewater and solutions of pristine nanoparticles after day-to-day conditions are applied a) sweat, b) chlorine, c) detergent and d) bleach. 


\section{Chapter 5: Transformation of Cerium Oxide nanoparticles during potential use scenarios from product incorporation to collection in the wastewater stream.}

Selvan Mohan, Kelsey Phillips, Juliska Princz, Maria C. DeRosa.

\subsection{Abstract}

The unique properties of nanoparticles lend themselves to incorporation within a range of commercial products. Cerium oxide nanoparticles, also known as nanoceria, exhibit radical scavenging ability, similar to superoxide dismutase. They also exhibit similar UV absorbance properties of current nanoparticles used in sunscreens (e.g., ZnO and $\mathrm{TiO}_{2}$ ). This suggests that cerium oxide nanoparticles may eventually find applications as additives in sunscreen and UV protective clothing. Inevitably, nanoparticles introduced into commercial products will eventually find their way into the environment. This study focuses on elucidating the transformations that nanoceria may undergo before releasing into the environment by modeling potential use and leaching scenarios. The cerium oxide nanoparticles were spiked into sunscreen to replicate a potential future use in consumer products. The nanoceria-loaded sunscreen was exposed to UV light, chlorinated water, and sweat to mimic potential environments where weathering could take place during use. The final weathering step took place by incubation in wastewater to mimic the impacts of leaching into wash water and traveling through the wastewater collection system. The transformations were analyzed by transmission electron microscopy (TEM) and energy-dispersive x-ray spectroscopy (EDS), which showed both aggregations of cerium oxide when exposed to chlorinated 
water, sweat, and wastewater, as well as elemental associations with sulfur and chlorine. X-ray photoelectron spectroscopy (XPS) was performed on the nanoparticles to confirm a chemical change of the $\mathrm{CeO}_{2}$ nanoparticles.

\subsection{Introduction}

Nanoparticles are defined as a matter between the size ranges of 1 to $100 \mathrm{~nm}$ that display unique, size-dependent properties, which allow for innovative applications ${ }^{1}$. They have found themselves increasingly used in commercial products such as in sunscreens for their UV protective properties ${ }^{2}$ and in fabrics for their anti-microbial activities ${ }^{3}$, among other applications.

One emerging nanomaterial of interest is that of cerium oxide nanoparticles

(nanoceria) due to their potential anti-oxidant properties ${ }^{4,5,6}$. Currently used in automotive catalytic converters and as fuel additives ${ }^{7}$, the radical scavenging properties of this material are thought to lead to its ability to act as an anti-oxidant and are opening up new applications $^{8}$. This property is similar to the activity of the superoxide dismutase enzyme, which takes oxygen radicals and catalyzes them to hydrogen peroxide, which can then be

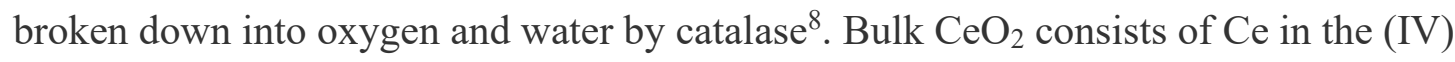
oxidation state. Due to the high surface area of $\mathrm{CeO}_{2}$ nanoparticles, some oxygen atoms leave the surface of the nanoparticles leaving behind reduced $\mathrm{Ce}^{3+}$ atoms ${ }^{9}$. The vacancy of oxygen atoms in the crystalline structure of nanoceria allows for the anti-oxidant property ${ }^{9}$. This anti-oxidant ability alone makes nanoceria a likely candidate for future research in consumer products, as well as medical applications ${ }^{10,11}$. Some possible 
medical applications of $\mathrm{CeO}_{2}$ nanoparticles are as neuroprotectants, cardiovascular disease inhibitors and magnetic resonance imaging (MRI) - contrast agents ${ }^{12-17}$.

Additionally, Cerium oxide has also been found to protect against UVA and UVB light as effectively as other nanoparticles currently being used in commercial products, such as titanium oxide and zinc oxide ${ }^{2}$. Moreover, $\mathrm{CeO}_{2}$ nanoparticles also block in the UVC spectrum at $230-260 \mathrm{~nm}^{18}$. Furthermore, $\mathrm{CeO}_{2}$ nanoparticles being transparent in the visible spectrum makes them ideal to be used in the cosmetic industry where products will appear natural on the $\operatorname{skin}^{19}$. Due to the combination of the properties above, $\mathrm{CeO}_{2}$ nanoparticles had been proposed to be a suitable alternative to $\mathrm{TiO}_{2}$ and $\mathrm{ZnO}$ in sunscreens ${ }^{19-21}$.

Given the growing interest in nanoceria, there is an increased potential for human exposure to these materials from the product as well as leaching into the aquatic and terrestrial compartments. The freshwater $\mathrm{CeO}_{2}$ nanoparticle content is expected to increase from $1 \mathrm{pg} / \mathrm{l}$ in 2017 to a few hundred $\mathrm{ng} / \mathrm{l}$ in $2050^{7}$. The quick adoption of nanoparticles into new products means that they may be introduced into the environment with little understanding of what effects they might cause ${ }^{22}$. The study of the toxicology of cerium oxide nanoparticles has generally met with positive results, where cerium oxide is less toxic than zinc oxide ${ }^{23}$ and as toxic as titanium oxide nanoparticles ${ }^{24}$. Nevertheless, some studies have been concerning. For example, 4 hours of continuous breathing at the $\mathrm{CeO}_{2}$ concentration of $641 \mathrm{mg} / \mathrm{m}^{3}$ causes inflammation of the lungs in $\mathrm{rat}^{25}$. Numerous other studies have concluded that the inflammation caused by the oxidative stress of $\mathrm{CeO}_{2}$ nanoparticles can cause cell apoptosis in humans ${ }^{26-28}$. On the 
other hand, exposing plants to $\mathrm{CeO}_{2}$ nanoparticles to investigate the potentially toxic effects, caused the plants to grow taller and have longer roots ${ }^{29}$.

As is the case of most nanotoxicology work, research that investigates the toxicity of cerium oxide nanoparticles examines pristine nanoparticles ${ }^{30,31}$. Possible transformations of nanoceria in the product life-cycle are not considered. Even if there are minimal harmful effects in their pristine form when observed in a laboratory setting $^{32,33}$, transformations could occur through the life-cycle of the nanoparticle that will change how the nanoparticles could interact with the environment or the living system.

With the life-cycle of commercial products in mind, it is our goal to examine the transformations of nanoceria that may take place during modeled use scenarios. An understanding of the compositional and morphological effects of daily use scenarios from product incorporation all the way to sewage water collection may allow for future nanotoxicological experiments to be performed with materials that better match what would be encountered in the environment.

Analysis of the nanoparticles was achieved through Transmission Electron Microscopy (TEM) for observations of the structural changes in the nanoparticles, such as aggregation and agglomeration. With Energy-dispersive X-ray spectroscopy (EDS), the analysis of the specific elemental associations with cerium oxide nanoparticles was achieved. X-ray photoelectron spectroscopy (XPS) was also used to show the chemical transformation of the nanoparticles (Figure 5-1). 

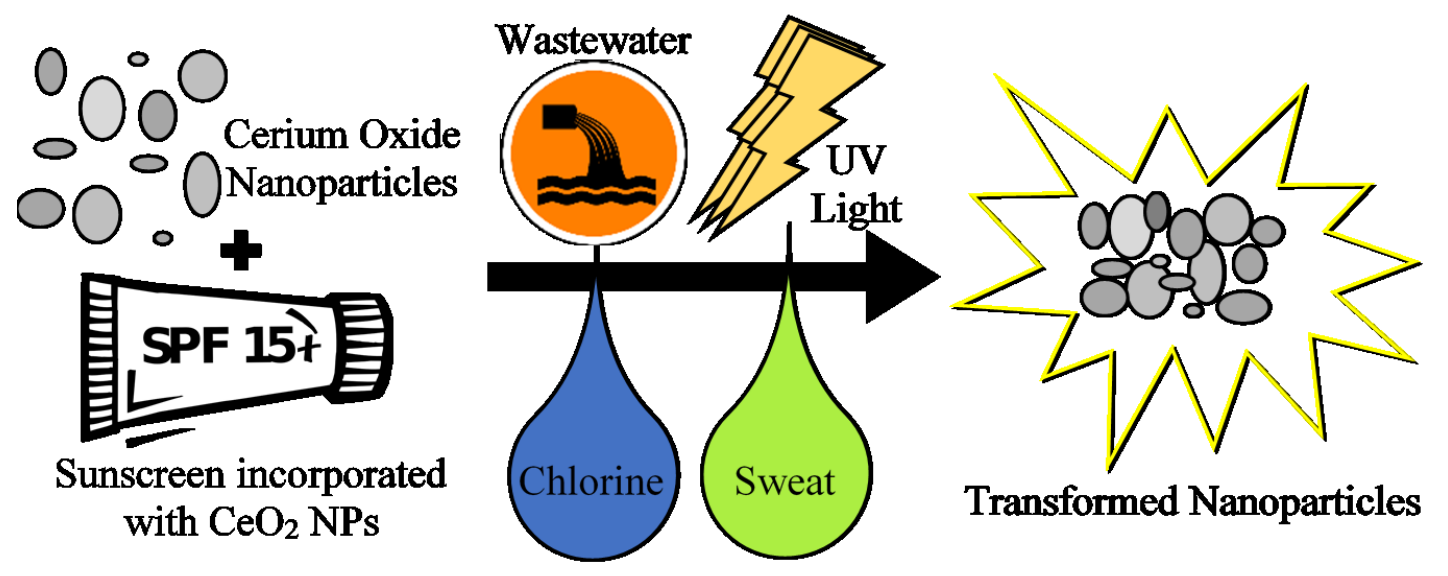

Figure 5-1: Transformation of nanoparticles (left) may occur when introduced into a consumer product and then exposed to chlorination, sweat, light, and wastewater (right).

\subsection{Materials and Methods}

All solutions were prepared with Millipore Milli-Q double deionized water at $18 \mathrm{M} \Omega$. Microcentrifuge tubes, reagents, and salts were purchased from Sigma-Aldrich. Carbon Film 300 Mesh Copper grids were purchased from Electron Microscopy Sciences (Hatfield, PA, USA). Aveeno Active Naturals sensitive skin mineral sunscreen (SPF 50) (Sunscreen A) and Neutrogena Ultra Sheer face sunscreen (SPF 60) (Sunscreen B) was purchased from Shoppers Drug Mart (Ottawa, Ontario, Canada). Exo-terra Sunray metal halide fixture with ballast and bulb (280-1000 $\mathrm{nm}$ wavelength, $70 \mathrm{~W})$ as well as a Large Zilla Fresh Air screen habitat was purchased from www.bigalspets.ca. Raw wastewater was obtained from the Robert O. Pickard Environmental Centre wastewater plant (Ottawa, Ontario, Canada). The Transmission Electron Microscope used was an FEI Tecnai G2F20. The EDS was an Aztec from Oxford-Instruments. The centrifuge used 
was a Sorvall Legend Micro 21r centrifuge from Thermofisher. The XPS used was Nova AXIS XPS from Kratos.

\subsubsection{Cleaning of Glassware with Aqua-Regia}

Aqua regia was used to clean all glassware before use. Nitric acid was mixed with $\mathrm{HCl}$ at a 1:3 ratio. After exposure of all glassware surfaces to aqua-regia, the glassware was then thoroughly rinsed with deionized water followed by distilled water and acetone before being left to dry. The used aqua-regia is neutralized with $\mathrm{NaOH}$ before disposal.

\subsubsection{Synthesis of Cerium Oxide Nanoparticles}

In a $50 \mathrm{~mL}$ round-bottom flask, $0.8618 \mathrm{~g}(2 \mathrm{mmol})$ of Cerium nitrate hexahydrate was added and dissolved in $10 \mathrm{~mL}$ of $\mathrm{dH}_{2} \mathrm{O}$. A stirring rod was added to the sample and the solution was heated to $70{ }^{\circ} \mathrm{C}$ with a stirring rate of $600 \mathrm{RPM}$. Then $5 \mathrm{~mL}$ of ammonium hydroxide, $3 \mathrm{M}$ was added to the solution, whereby a cloudy white precipitate immediately formed. A syringe connected to an air bubbler was inserted into the solution, and the air was bubbled into the solution. The precipitate changed from white to purple in colour. Parafilm was wrapped over the top of the flask to prevent moisture from escaping. The sample was left to stir under heat for approximately 12 hours and recovered as a yellowish-white solution. This synthesis method was adopted and modified from that proposed by Huey-Ing Chen and Hung-Yi Chang ${ }^{34}$. 

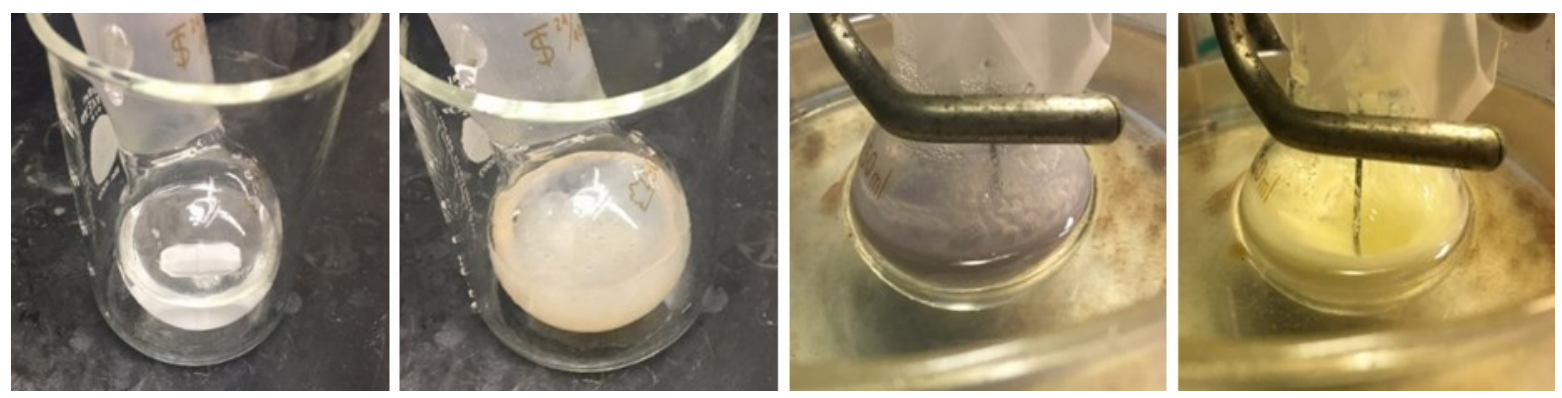

Figure 5-2: (left to right) Solution of Cerium nitrate and water; 10 seconds after the ammonium hydroxide added to the solution; Colour change to purple minutes after stirring the solution; Colour change to yellowish-white colour overnight.

\subsubsection{Washing of Cerium Oxide Nanoparticles}

The solution was divided into $2 \mathrm{~mL}$ centrifuge tubes. Each tube was centrifuged at 10,000 RPM for 10 minutes, and the supernatant was discarded. Ethanol was added to fill the centrifuge tubes, and then the samples were put into a water sonicator and vortexed until the nanoparticles were resuspended. The sample was then centrifuged at 10,000 RPM for 10 minutes. Deionized water was then added to fill the centrifuge tubes as the above process. This cycle of ethanol and water washes was repeated two more times. Finally, ethanol was added to the sample, which was then sonicated and vortexed to suspend the nanoparticles in the solution ${ }^{34}$.

After the final washing step, the cerium oxide nanoparticles were suspended in ethanol solution. Samples were transferred to a $50 \mathrm{~mL}$ round-bottom flask. The roundbottom flask was attached to a rotovap set at $40{ }^{\circ} \mathrm{C}$ at $70 \mathrm{RPM}$ for 1 hour to dry the samples. This synthesis yielded $81.9 \% \mathrm{CeO}_{2}$. The flask was then wrapped in aluminum foil and stored at room temperature. 


\subsubsection{Preparation of Ceria-doped Sunscreen}

$3.00 \mathrm{~g}$ of sunscreens (Sunscreen A and B) were added into respective $50 \mathrm{~mL}$ round bottom flasks. Then $0.090 \mathrm{~g}$ of synthesized nanoceria were added to each sample. This equates to $3.0 \% \mathrm{w} / \mathrm{w}$ of nanoparticles, which is equal to the zinc oxide content in Sunscreen A. A stir-bar was placed into the round bottom flask before capping. The sunscreen and nanoceria were left to stir for 12 hours.

\subsubsection{Nanoparticle Extraction}

\subsubsection{Extraction Through Incineration}

$30 \mathrm{mg}$ Cerium Oxide Nanoparticles and $0.50 \mathrm{~g}$ nanoparticle-impregnated sunscreens were added to separate $10 \mathrm{~mL}$ Pyrex beakers. They were placed into an incinerator at $600{ }^{\circ} \mathrm{C}$ for 3 hours. After the 3 hours, the samples were allowed to cool for

6 hours inside the incinerator ${ }^{35}$. The beakers were then removed to the incinerator and 1.5 $\mathrm{mL}$ of $1.0 \% \mathrm{HCl}$ solution was added to each beaker. The solution was stirred to suspend the nanoparticles into the solution.

\subsubsection{Water Extraction}

30 mg Cerium Oxide Nanoparticles and 0.50 g nanoparticle-impregnated sunscreens were added to separate $50 \mathrm{~mL}$ centrifuge tubes. $12.5 \mathrm{~mL}$ of $\mathrm{dH}_{2} \mathrm{O}$ was added to each tube and put onto a shaker set at $160 \mathrm{RPM}$ at $37^{\circ} \mathrm{C}$ for 24 hours. The suspension was used for analysis. 


\subsubsection{Liquid-Liquid Extraction}

For pure nanoceria, Sunscreen A and Sunscreen B, 0.0489 g, 0.1109 g, and $0.1060 \mathrm{~g}$, respectively, were added to $2.0 \mathrm{~mL}$ centrifuge tubes. Then, $2 \mathrm{~mL}$ of hexanes was added to each centrifuge tube, which was then sonicated and vortexed until dissolved. The tubes were centrifuged at 7000 RPM for 2 minutes, with the supernatant decanted. Next, $2 \mathrm{~mL}$ of ethanol was added to each centrifuge and sonicated and vortexed until dissolved. Then the samples were centrifuged at 7000 RPM for 2 minutes. This process was repeated for a total of three times with ethanol. The same procedure was used with $\mathrm{dH}_{2} \mathrm{O}$ and then finally with acetone, both repeated three times ${ }^{36}$. For the final acetone step, the extracted samples were added to the copper TEM grid for analysis.

\subsubsection{Weathering of nanoparticles}

\subsubsection{1 $\underline{\text { Sweat }}$}

Sweat solutions were made in a $50 \mathrm{~mL}$ Erlenmeyer flask, with $0.2700 \mathrm{~g}$ of NaCl, $0.0325 \mathrm{~g}$ Urea added to the flask before $25 \mathrm{~mL}$ of $\mathrm{dH}_{2} \mathrm{O}$, and then $23.3 \mu \mathrm{L}$ of lactic acid was added to the solution ${ }^{35}$. Once the sweat solution was prepared, doped-sunscreens were added to the flask and allowed to incubate at $160 \mathrm{RPM}$ at $37^{\circ} \mathrm{C}$ overnight.

\subsubsection{Chlorine}

Chlorinated water solutions were prepared by adding $25 \mathrm{~mL}$ of $\mathrm{dH}_{2} \mathrm{O}$ to a $50 \mathrm{~mL}$ Erlenmeyer flask and then pipetting $2.0 \mu \mathrm{L}$ of sodium hypochloride into the water ${ }^{37}$. 
Once prepared, the doped-sunscreen was added to the flask, and the solution was then incubated at $160 \mathrm{RPM}$ at $37^{\circ} \mathrm{C}$ overnight.

\subsubsection{Wastewater}

Wastewater was obtained from the Robert O. Pickard Environmental Centre. The raw wastewater was used within 6 hours of collection from the sewage treatment plant, and the content of raw wastewater was analyzed within 48 hours. Sewage water content can be found in supporting information (Table S1)

To each of the samples being tested, (pure Ceria, Sunscreen A and Sunscreen B) a 1:1 mixture of $\mathrm{dH}_{2} \mathrm{O}$, and wastewater were added to the samples (200 mg sample, $1 \mathrm{~mL}$ $\mathrm{ddH}_{2} \mathrm{O}, 1 \mathrm{~mL}$ wastewater). The samples were vortexed and then allowed to incubate at $160 \mathrm{RPM}$ and $37^{\circ} \mathrm{C}$ for 24 hours. TEM grids were then prepared for analysis.

\subsubsection{UV Light Exposure}

The samples, whether doped-sunscreen or the controls, were spread onto a glass Petri-dish and set 0.6 meters under the lamp for 24 hours of exposure time. The samples were removed the following day.

\subsubsection{Preparation of Samples for TEM, EDS, and XPS Analysis}

All samples were vortexed, and $6 \mu \mathrm{L}$ of the sample was added onto the Carbon Film 3000 Mesh, copper grid and allowed to sit for 1 minute. Then, the sample was removed from the grid by pipetting. Another $6 \mu \mathrm{L}$ of the sample was added to the TEM 
grid, and the residual sample was removed after 1 minute for a second time. The grids were then left to air dry for 2 hours before placing it into the TEM. The EDS of the sample was analyzed through an attachment to the TEM. The area of analysis of the beam is highlighted in the images with a red circle.

The XPS spectrum was obtained using a Kratos Nova AXIS spectrometer equipped with an $\mathrm{Al} \mathrm{X}$-ray source, using $\mathrm{AlK}_{\alpha}$ radiation, charge neutralizer, and a delayline detector (DLD). The sample used for XPS were all in the liquid form wherein $10 \mu \mathrm{L}$ of the sample was applied onto a glass coverslip. The sample was left to air dry for two hours. Once dried, another $10 \mu \mathrm{L}$ of the sample was applied to the exact same spot and again left to air dry for two hours. This step was repeated 5 times or more until a visible spot was observed on the glass coverslip.

\subsection{Results and Discussion}

\subsubsection{Synthesis of Pure Ceria}

The synthesis method used in this paper had been adopted and modified from that proposed by Huey-Ing Chen and Hung-Yi Chang ${ }^{34}$. Variations of temperature and ambient $\mathrm{O}_{2} \%$ were tested in their synthesis of $\mathrm{CeO}_{2}$ nanoparticles. Based on the particle size and shape uniformity, the synthesis at $70{ }^{\circ} \mathrm{C}$ was chosen for this work. The $\% \mathrm{O}_{2}$ that was bubbled into the reaction was $21 \%$ (ambient air). The first attempt at synthesis yielded nanoparticles with very similar morphology to that found in the paper. The size distribution analyzed with DLS showed two distinct particle sizes (Figure 5-3). The DLS peaks represent the hydrodynamic radius that affects the light scattering ${ }^{38}$. The $\mathrm{CeO}_{2}$ 
nanoparticle size is smaller than its hydrodynamic radius and a broad peak can indicate the existence of nanoclusters ${ }^{39,40}$.
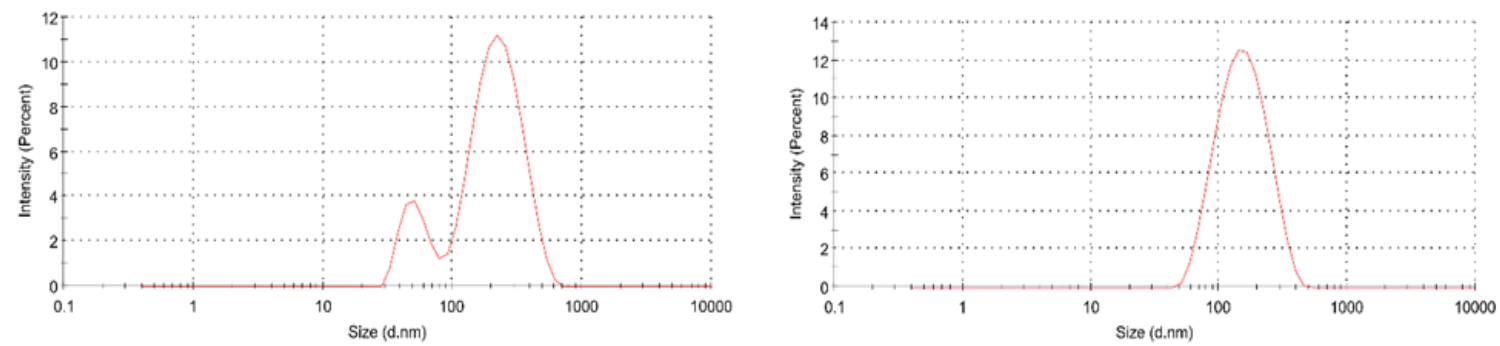

Figure 5-3: DLS distribution nanoceria from initial synthesis (left) and modified synthesis technique (right).

To improve the size distribution, the vigorousness of the bubbling was varied.

Continuous bubbling (approximately 10 bubbles per second) helped to synthesize smaller nanoparticles, but a slower bubbling at approximately 2 bubbles per second with an increase of reaction stir time gave a single distribution peak on the DLS chart and a size range of $12 \pm 7 \mathrm{~nm}$ found from the TEM images. Moreover, the EDS and XPS spectra found in Figure 5-4 and Figure 5-6, respectively, confirm the synthesis of pure $\mathrm{CeO}_{2}$. It must be noted that $\mathrm{CeO}_{2}$ nanoparticles tend to aggregate and form clusters ${ }^{39-41}$, which is supported by a zeta potential of $21.3 \pm 6.1 \mathrm{mV}$. Nanoparticles with zeta potential value between $+25 \mathrm{mV}$ and $-25 \mathrm{mV}$ eventually aggregate due to interparticle interactions, including van der Waals and hydrophobic interactions, and hydrogen bonding ${ }^{42}$. 


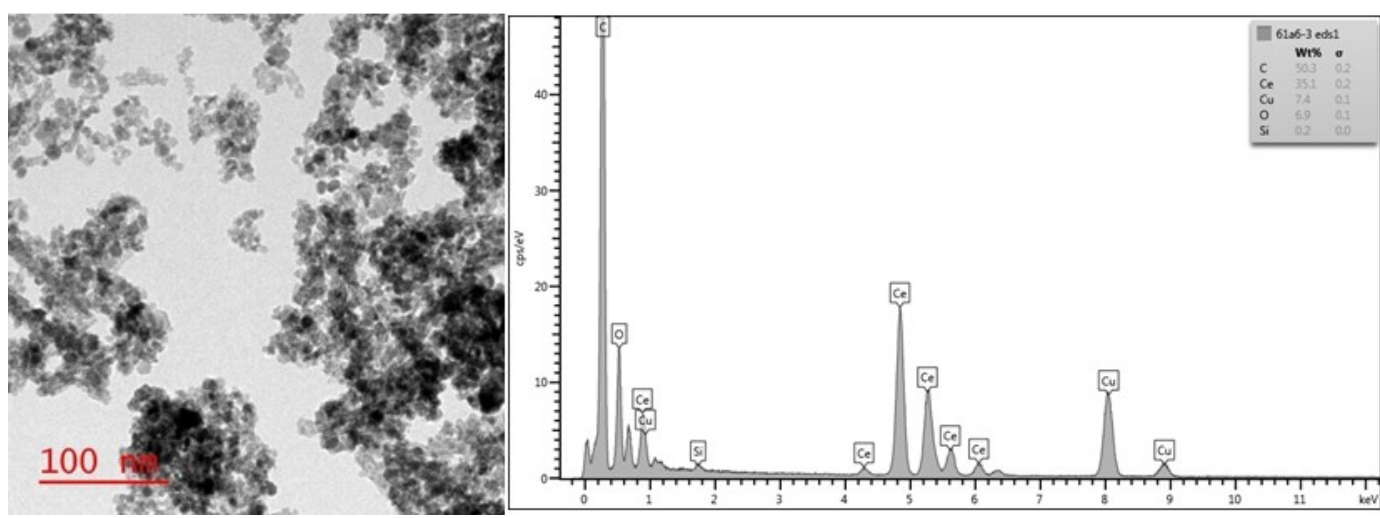

Figure 5-4: TEM and EDS image of synthesized $\mathrm{CeO}_{2}$ nanoparticles. A large-scale view image can be found in Figure S 5-1 of the supporting information.

\subsubsection{Doped-Sunscreens with nanoceria}

The exceptional property of $\mathrm{CeO}_{2}$ in absorbing $\mathrm{UV}$ rays as well and being a nanoparticle that can be an excellent antioxidant makes it an ideal candidate for use in sunscreen. This will be a use scenario that may lead to the introduction of a large amount of these nanoparticles into the environment. Given that these particles are not currently used in this application, this study represents an opportunity to provide some foresight on a potential future use-scenario and any anticipated morphological and chemical changes that may result.

Aveeno sensitive skin sunscreen (Sunscreen A) and Neutrogena Ultrasheer sunscreen (Sunscreen B) were impregnated with $\mathrm{CeO}_{2}$ nanoparticles (Table 5-1). Both sunscreens are commonly used in commercial brands. Sunscreen A contains both Titanium oxide and Zinc oxide nanoparticles, but Sunscreen B does not contain any metal-oxide nanoparticles. This difference makes them an ideal candidate to compare the stability of $\mathrm{CeO}_{2}$ nanoparticles in the sunscreen matrix. 
After adding the nanoparticles to the sunscreens, the sunscreens were analyzed after 24 hours, 1-week, 2-weeks, and 4-weeks. The results are shown in Table 5-1. The integration of nanoparticles into the sunscreen matrix was observed at the 24-hour mark. The chemical and physical morphology was not observed to change with time. During the analysis of TEM samples, no agglomerated nanoceria was located, further confirming the successful integration of $\mathrm{CeO}_{2}$ nanoparticles into the sunscreen matrix. All further experiments on the transformation of cerium oxide nanoparticles were carried out on sunscreen that had been resting for at least one week after impregnation. 
Table 5-1: TEM images and EDS spectrum of $\mathrm{CeO}_{2}$ nanoparticles after impregnation into sunscreen A and sunscreen B at 24 hours, 1-week, and 2-week duration to show the stability of the nanoparticles in the sunscreen matrix.

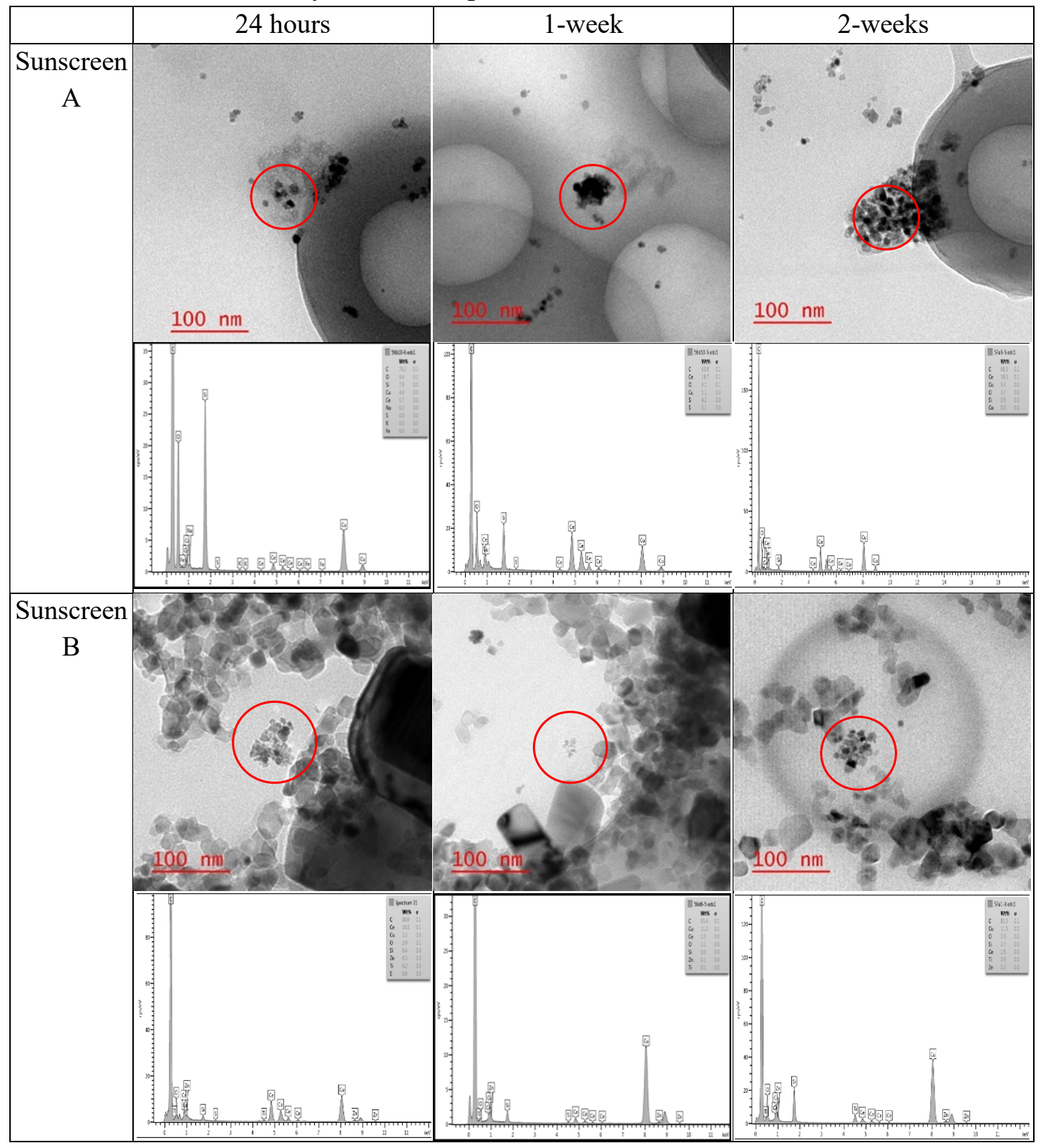

The sunscreen was also analyzed after 4-weeks. Instead of just dissolving the sunscreen in $\mathrm{dH}_{2} \mathrm{O}$, the $\mathrm{CeO}_{2}$ nanoparticles were also extracted from the sunscreen by incineration and liquid-liquid extraction. Despite reports that incineration is not destructive to $\mathrm{CeO}_{2}$ nanoparticles ${ }^{43}$, the silicone in sunscreens formed large hetero- 
agglomerates with the nanoparticles (Table S 5-2, Supporting Information). Using polar and non-polar solvent to dissolve the sunscreen followed by centrifugation to retain the heavier metal nanoparticles provided a sample with less amount of impurities; however, similar results were obtained by just diluting the nanoparticles with $\mathrm{dH}_{2} \mathrm{O}$ (Table $\mathbf{S ~ 5 - 2}$, Supporting Information).

The analysis after 4-weeks has also shown the integration of the nanoparticles into sunscreen, but more importantly, there were no clear indications of chemical transformation observed from the EDS analysis (Table S 5-2, Supporting Information). When other elements are observed with cerium, the background beside the nanoparticles had shown the existence of the same elements. Moreover, these elements were not observed consistently throughout the sample, indicating that EDS had shown these elements due to its proximity to cerium but not because of a chemical transformation of cerium. As a result, we can conclude that this product incorporation has minimal impact on the morphology and composition of nanoceria.

\subsubsection{Weathering of $\mathrm{CeO}_{2}$ nanoparticles through day-to-day use, $\mathrm{UV}$ and wastewater exposure}

Commercial products containing nanoparticles will go through specific use scenarios. In the case of sunscreen that is used outdoors, exposure to sweat on the skin, as well as chlorine from pool water, will be a common occurrence. Sunscreen A, Sunscreen $\mathrm{B}$, and pristine nanoparticles were subjected to incubation with sweat and chlorine for 24hours to mimic an extreme use case. 
Table 5-2: TEM images and EDS spectrum of $\mathrm{CeO}_{2}$ nanoparticles after incubation of sunscreen A, sunscreen B, and pristine nanoparticles in sweat and chlorine for 24 hours to mimic day-to-day use. Elemental association with chlorine was observed on all samples treated with sweat through EDS.

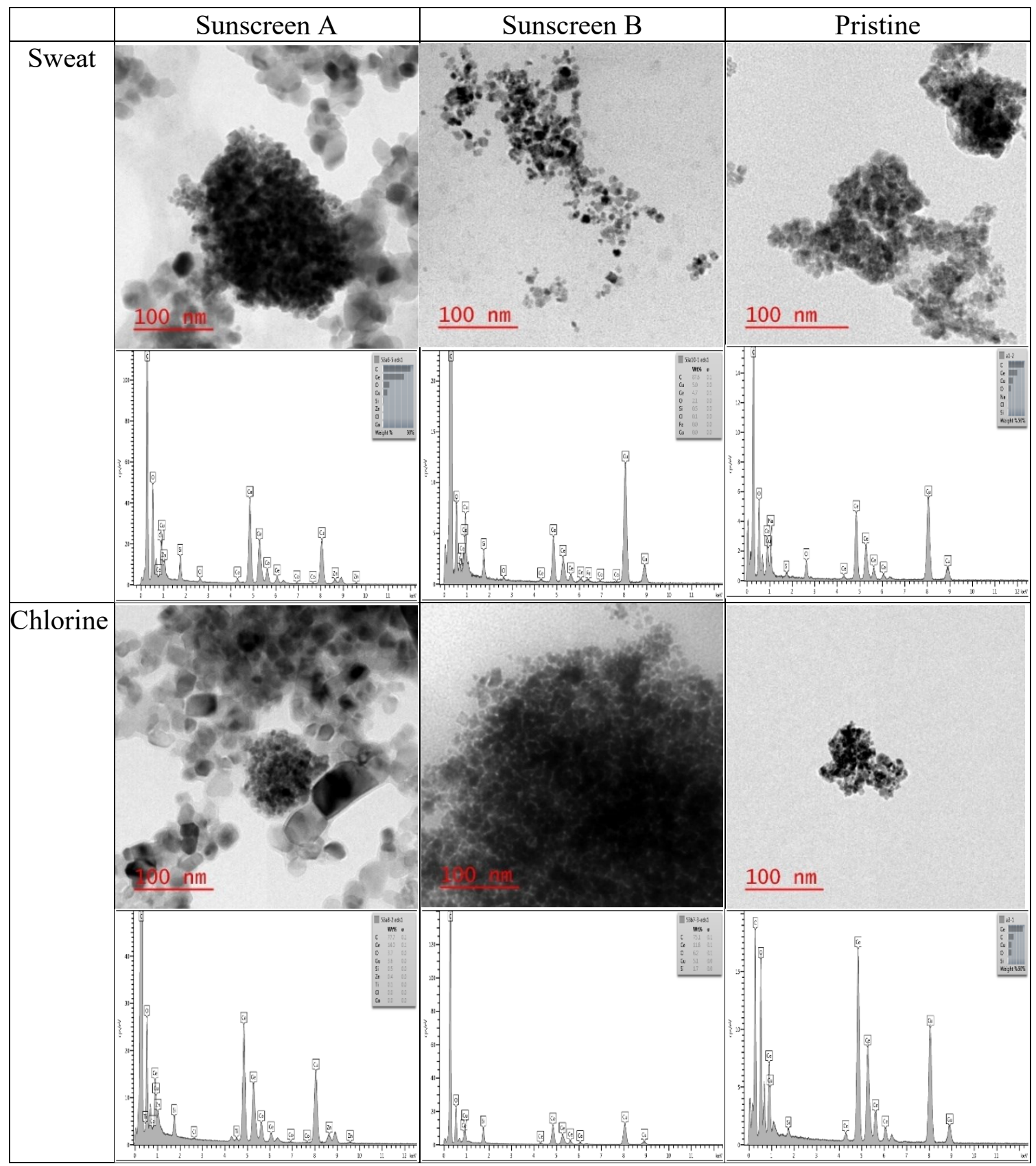

Upon incubating the sunscreens and pristine nanoparticles in sweat and chlorine, a more pronounced aggregation of the nanoparticles as large dense clusters was observed 
throughout all the TEM images (Table 5-2). Studying the elemental analysis of the samples through EDS showed chlorine association to all the $\mathrm{CeO}_{2}$ nanoparticles of samples incubated with sweat. The association with chlorine was only observed in sweat due to its low $\mathrm{pH}^{35}$, which is known to cause transformation in $\mathrm{CeO}_{2}$ nanoparticles ${ }^{44}$. To confirm the chemical transformation of $\mathrm{CeO}_{2}$ nanoparticles, XPS analysis was performed (Figure 5-6).

The physical and chemical transformation of $\mathrm{CeO}_{2}$ nanoparticles could also be affected by exposure to UV light. Another set of samples of sunscreen A, sunscreen B, and pristine nanoparticles were incubated in sweat and chlorine while under a full spectrum lamp ${ }^{45}$. 
Table 5-3: TEM images and EDS spectrum of $\mathrm{CeO}_{2}$ nanoparticles after incubation of sunscreen A, sunscreen B, and pristine nanoparticles in sweat and chlorine for 24 hours while under UV light to mimic day-to-day use. Elemental association with chlorine was observed on all samples treated with sweat + UV through EDS. Elemental association with sulfur was observed in both sunscreens treated with sweat + UV.

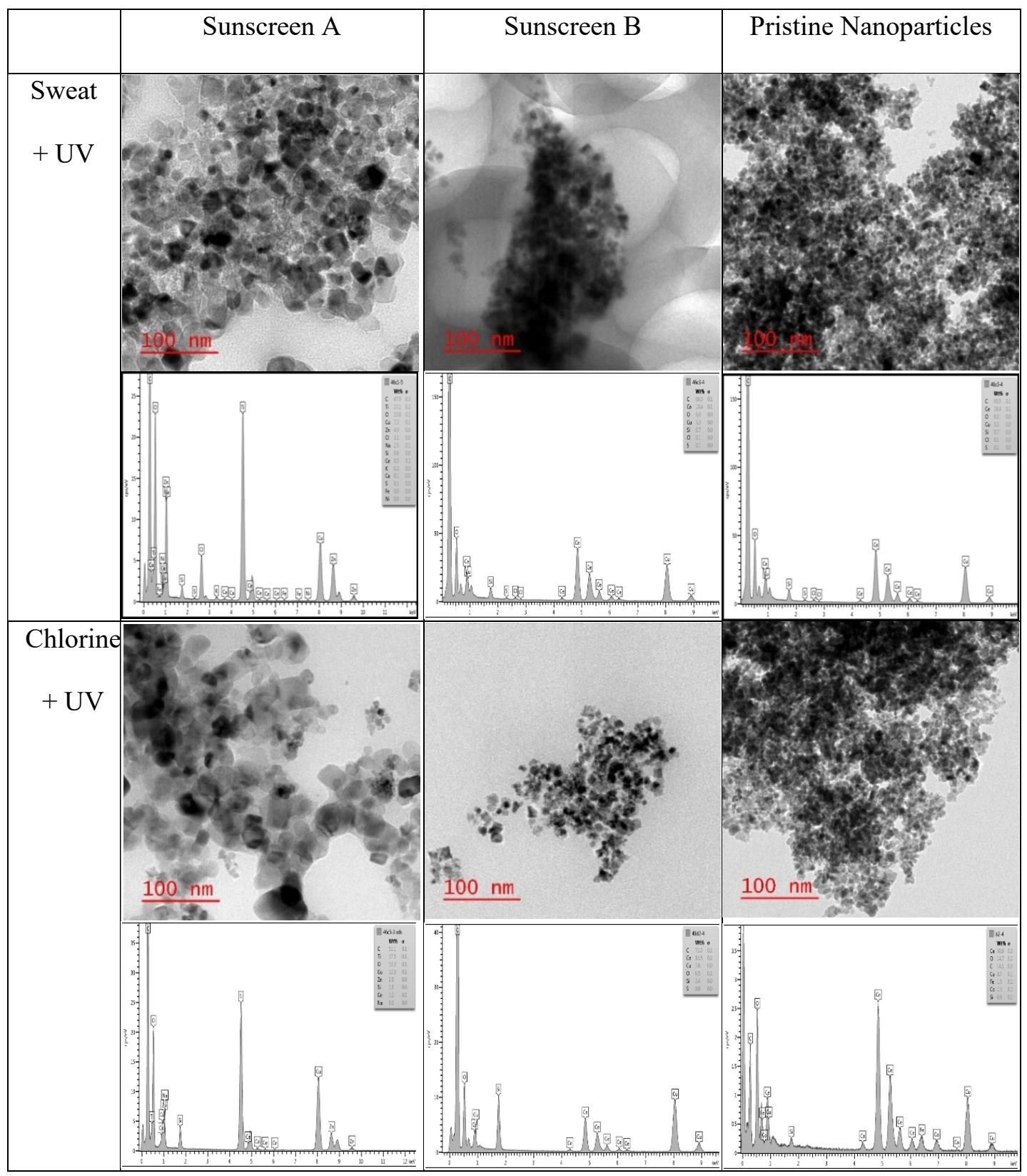

TEM images in Table 5-3 showed no difference in the aggregation of the nanoparticles when compared to samples that were not exposed to UV light. However, 
EDS analysis of the sweat and UV treated sunscreens showed association with sulfur in addition to chlorine that was observed with no UV exposure. The formation of sulfate groups on the surface of $\mathrm{Ce}_{2} \mathrm{O}$ NPs had been previously reported ${ }^{44} \cdot \mathrm{CeO}_{2}$ had also been shown to form cerium sulfide $\left(\mathrm{Ce}_{2} \mathrm{~S}_{3}\right)$ when exposed to gaseous sulfur ${ }^{46}$ (Eqs. 1 below)

$\mathrm{CeO}_{2}+3 \mathrm{H}_{2} \mathrm{~S}+\mathrm{H}_{2} \leftrightarrow \mathrm{Ce}_{2} \mathrm{~S}_{3}+4 \mathrm{H}_{2} \mathrm{O}$

The sulfur was only present in the sunscreen samples due to the presence of phenylbenzimidazole sulfonic acid which is a common compound found in sunscreens due to the ability to absorb at the UV-B wavelength ${ }^{47}$. The chemical transformation of $\mathrm{CeO}_{2}$ nanoparticles was confirmed by performing XPS analysis (Figure 5-6).

After the sunscreens are used, they would end up in the water treatment plant through showering or draining of the pool water. On their way to the treatment plant, they could come into contact with a very complex chemical environment. To test the physical and chemical transformations of the next part of $\mathrm{CeO}_{2}$ lifecycle, the sunscreens and pristine nanoparticles were incubated with wastewater. 


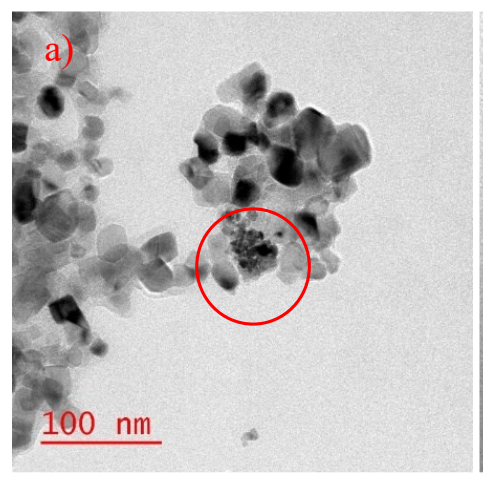

b)

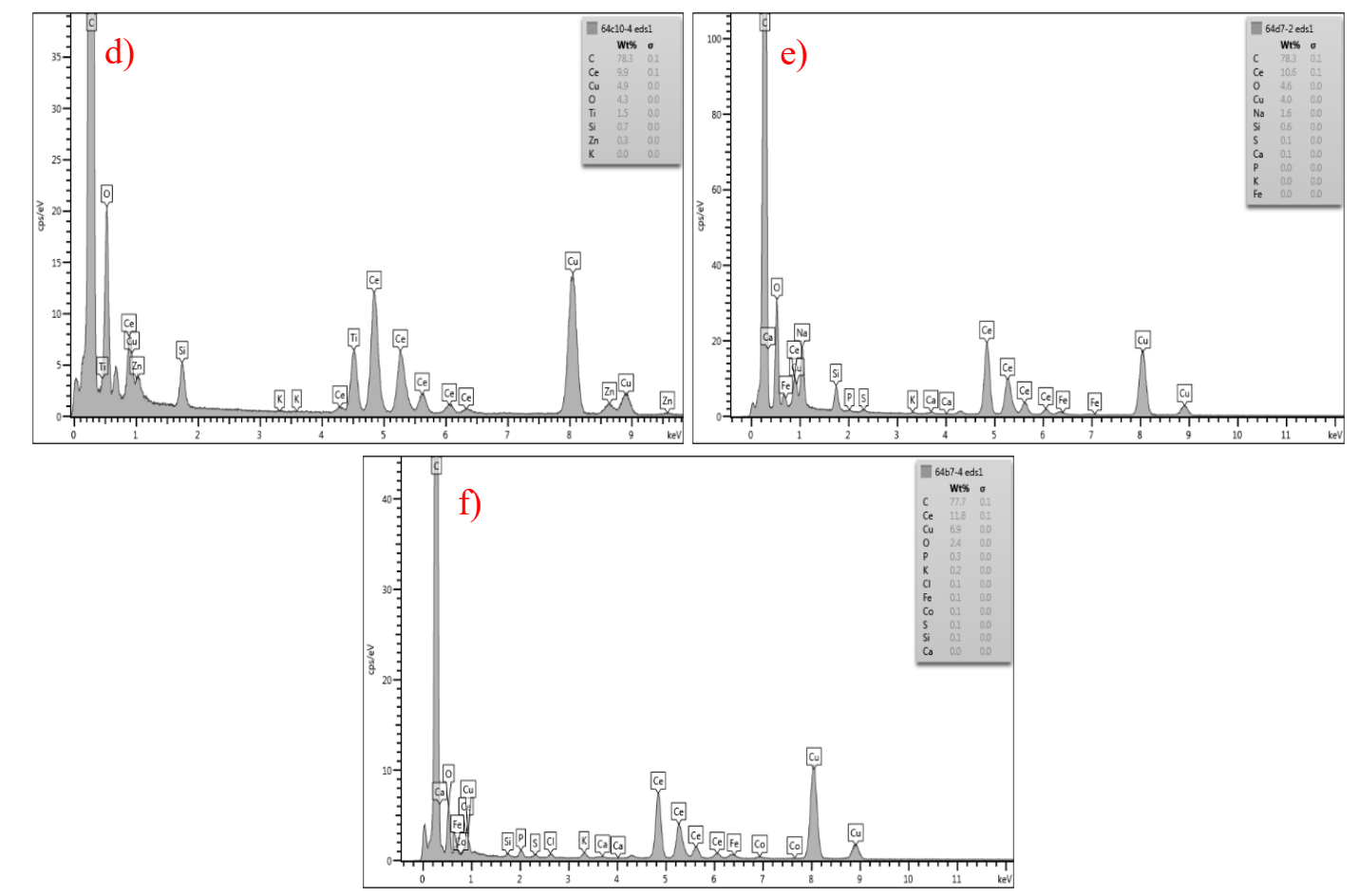

$100 \mathrm{~nm}$

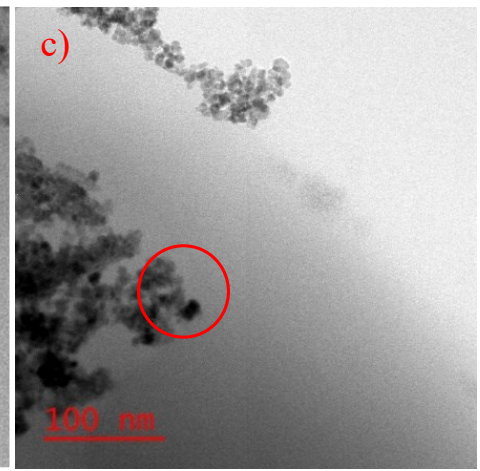

Figure 5-5: TEM images and EDS peaks of Sunscreen A (a,d), Sunscreen B (b,e), and Pristine $\mathrm{CeO}_{2}$ nanoparticles (c,f), after incubated with sewage water for 24 hours to mimic wastewater collection. Chlorine and sulfur were found in all samples via EDS analysis.

Nanoparticle aggregation into clusters was again observed in the TEM images of all the samples incubated in sewage water. Moreover, all samples show chemical associations with sulfur and chlorine. Agglomeration of $\mathrm{CeO}_{2}$ nanoparticles was also observed as large particle formations in wastewater weathered samples (Figure 5-5 (b), 
red circle). The agglomeration can be caused by the dissolution of the nanoparticles, followed by precipitation during the process of TEM sample preparation ${ }^{44,48}$. The outcome for wastewater exposure alone was enough to show the greatest degree of chemical changes in the nanoparticles. To confirm that the nanoparticles were indeed being chemically transformed by the various exposures, XPS analysis was performed on the pristine nanoparticles and compared to that of pristine nanoparticles exposed to wastewater (Figure 5-6). 


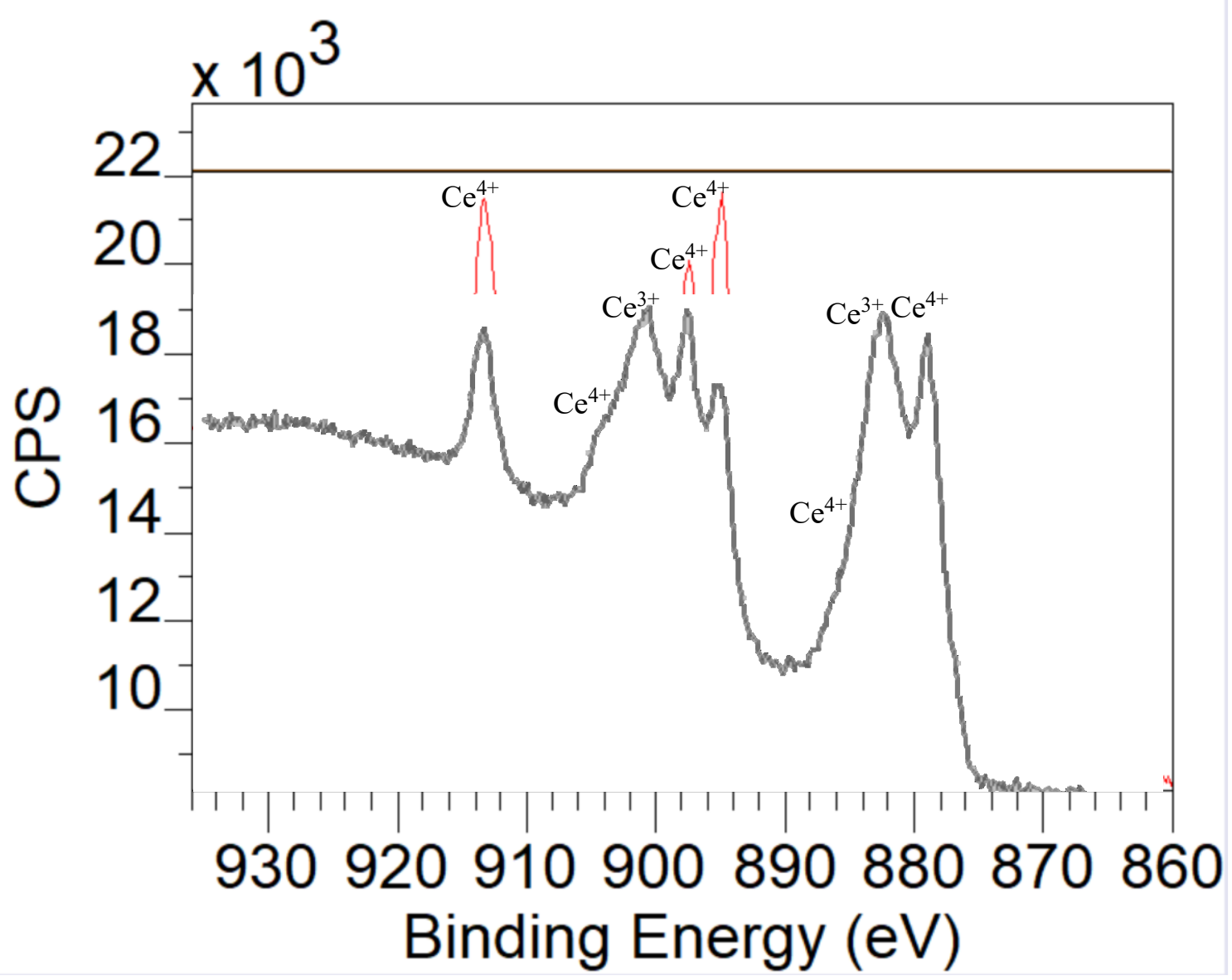

Figure 5-6: XPS spectrum (860- $935 \mathrm{eV}$ ) of cerium peaks in synthesized pristine $\mathrm{CeO}_{2}$ nanoparticles (red) compared to $\mathrm{CeO}_{2}$ nanoparticles incubated in wastewater for 24-hours (grey). The oxidation state of cerium represented by the peaks are labeled directly over the peaks ${ }^{49-51}$.

XPS analysis was performed to show the chemical change that could occur in the $\mathrm{CeO}_{2}$ nanoparticles. By comparing $\mathrm{Ce}^{3+}$ to $\mathrm{Ce}^{4+}$ ratios, the change of the cerium oxidation state can be determined. Based on Figure 5-6, the wastewater sample shown in grey has two peaks that are more intense than in the pristine nanoparticles The greater extent of $\mathrm{Ce}^{3+}$ oxidation state after wastewater incubation could be easily seen with contribution from peaks at $903 \mathrm{eV}$ and $885 \mathrm{eV}$. Thus, the wastewater exposed nanoparticles have a higher $\mathrm{Ce}^{3+}$ to $\mathrm{Ce}^{4+}$ ratio compared to pristine nanoparticles ${ }^{49,52}$. The reduction of $\mathrm{Ce}^{4+}$ to 
$\mathrm{Ce}^{3+}$ was also found to occur in sewage sludge ${ }^{53,54}$. The increase of the $\mathrm{Ce}^{3+}$ oxidation state further supports the chemical association with chlorine and sulfur in forming $\mathrm{CeCl}_{3}$ and $\mathrm{Ce}_{2} \mathrm{~S}_{3}$ respectively. $\mathrm{CeO}_{2}$ nanoparticles had been shown to be slightly soluble in water making the formation of cerium salts more likely $y^{48,55}$.

To conclude, despite the qualitative approach of this study, $\mathrm{CeO}_{2}$ nanoparticles have been significantly transformed physically and chemically during the simulated dayto-day use and wastewater exposure processes. Despite the aggregation and reduction of $\mathrm{CeO}_{2}$ nanoparticles, there is no evidence suggesting the full dissolution of the nanoparticles. Furthermore, the most significant transformation for $\mathrm{CeO}_{2}$ nanoparticles was observed during the final weathering with wastewater exposure. Having a higher ratio of $\mathrm{Ce}^{3+}$ to $\mathrm{Ce}^{4+}$ in $\mathrm{CeO}_{2}$ nanoparticles can more toxic to algal cells ${ }^{56}$. Consequently, the incubation of pristine $\mathrm{CeO}_{2}$ nanoparticles in wastewater for 24-hours will produce weathered nanoparticles that can be used for environmental studies to accurately understand the environmental impact of these nanoparticles. In the future, other potential commercial use scenarios for $\mathrm{CeO}_{2}$ nanoparticles will be investigated. Moreover, studies on the morphological and compositional transformation of $\mathrm{CeO}_{2}$ nanoparticles during the wastewater and biosolid treatment process will be conducted. 


\subsection{References}

1. Foss Hansen, S., Larsen, B. H., Olsen, S. I. \& Baun, A. Categorization framework to aid hazard identification of nanomaterials. Nanotoxicology 1, 243-250 (2007).

2. Zholobak, N. M. et al. UV-shielding property, photocatalytic activity and photocytotoxicity of ceria colloid solutions. J. Photochem. Photobiol. B Biol. 102, 32-38 (2011).

3. Zhang, F., Wu, X., Chen, Y.\& Lin, H. Application of silver nanoparticles to cotton fabric as an antibacterial textile finish. Fibers Polym. 10, 496-501 (2009).

4. Karakoti, A. S. et al. Nanoceria as antioxidant: Synthesis and biomedical applications. JOM 60, 33-37 (2008).

5. Das, S. et al. Cerium oxide nanoparticles: applications and prospects in nanomedicine. Nanomedicine 8, 1483-1508 (2013).

6. Xu, C. \& Qu, X. Cerium oxide nanoparticle: a remarkably versatile rare earth nanomaterial for biological applications. NPG Asia Mater. 6, e90 (2014).

7. Giese, B. et al. Risks, Release and Concentrations of Engineered Nanomaterial in the Environment. Sci. Rep. 8, 1565 (2018).

8. Korsvik, C., Patil, S., Seal, S. \& Self, W. T. Superoxide dismutase mimetic properties exhibited by vacancy engineered ceria nanoparticles. Chem. Commun. 1056-1058 (2007).

9. Deshpande, S., Patil, S., Kuchibhatla, S. V. \& Seal, S. Size dependency variation in lattice parameter and valency states in nanocrystalline cerium oxide. Appl. Phys. Lett. 87, 133113 (2005).

10. Yabe, S. Cerium oxide for sunscreen cosmetics. J. Solid State Chem. 171, 7-11 (2003).

11. Das, S. et al. The induction of angiogenesis by cerium oxide nanoparticles through the modulation of oxygen in intracellular environments. Biomaterials 33, 77467755 (2012).

12. Das, M. et al. Auto-catalytic ceria nanoparticles offer neuroprotection to adult rat spinal cord neurons. Biomaterials 28, 1918-1925 (2007).

13. Niu, J., Azfer, A., Rogers, L., Wang, X. \& Kolattukudy, P. Cardioprotective effects of cerium oxide nanoparticles in a transgenic murine model of cardiomyopathy. Cardiovasc. Res. 73, 549-559 (2007).

14. Chen, J., Patil, S., Seal, S. \& Mcginnis, J. F. Rare earth nanoparticles prevent 
retinal degeneration induced by intracellular peroxides. 1, 142-150 (2006).

15. Eriksson, P. et al. Cerium oxide nanoparticles with antioxidant capabilities and gadolinium integration for MRI contrast enhancement. Sci. Rep. 8, 6999 (2018).

16. Schubert, D., Dargusch, R., Raitano, J. \& Chan, S.-W. Cerium and yttrium oxide nanoparticles are neuroprotective. Biochem. Biophys. Res. Commun. 342, 86-91 (2006).

17. Niu, J., Wang, K. \& Kolattukudy, P. E. Cerium oxide nanoparticles inhibit oxidative stress and nuclear factor- $\mathrm{\kappa B}$ activation in $\mathrm{H} 9 \mathrm{c} 2$ cardiomyocytes exposed to cigarette smoke extract. J. Pharmacol. Exp. Ther. 338, 53-61 (2011).

18. Medalia, A. \& Byrne, B. Spectrophotometric Determination of of Cerium (IV). Anal. Chem. 23, 453-456 (1951).

19. Yabe, S. \& Sato, T. Cerium oxide for sunscreen cosmetics. J. Solid State Chem. 171, 7-11 (2003).

20. Li, R. et al. UV-shielding properties of zinc oxide-doped ceria fine powders derived via soft solution chemical routes. Mater. Chem. Phys. 75, 39-44 (2002).

21. Caputo, F. et al. Cerium oxide nanoparticles, combining antioxidant and UV shielding properties, prevent UV-induced cell damage and mutagenesis. Nanoscale 7, 15643-15656 (2015).

22. Pelletier, D. A. et al. Effects of engineered cerium oxide nanoparticles on bacterial growth and viability. Appl. Environ. Microbiol. 76, 7981-9 (2010).

23. Xia, T. et al. Comparison of the Mechanism of Toxicity of Zinc Oxide and Cerium Oxide Nanoparticles Based on Dissolution and Oxidative Stress Properties. ACS Nano 2, 2121-2134 (2008).

24. García, A. et al. Acute toxicity of cerium oxide, titanium oxide and iron oxide nanoparticles using standardized tests. Desalination 269, 136-141 (2011).

25. Srinivas, A., Rao, P. J., Selvam, G., Murthy, P. B. \& Reddy, P. N. Acute inhalation toxicity of cerium oxide nanoparticles in rats. Toxicol. Lett. 205, 105-115 (2011).

26. Thill, A. et al. Cytotoxicity of $\mathrm{CeO} 2$ Nanoparticles for Escherichia coli. PhysicoChemical Insight of the Cytotoxicity Mechanism. Environ. Sci. Technol. 40, 61516156 (2006).

27. Park, E.-J., Choi, J., Park, Y.-K. \& Park, K. Oxidative stress induced by cerium oxide nanoparticles in cultured BEAS-2B cells. Toxicology 245, 90-100 (2008).

28. Eom, H.-J. \& Choi, J. Oxidative stress of $\mathrm{CeO} 2$ nanoparticles via p38-Nrf-2 signaling pathway in human bronchial epithelial cell, Beas-2B. Toxicol. Lett. 187, 
$77-83$ (2009).

29. Morales, M. I. et al. Toxicity Assessment of Cerium Oxide Nanoparticles in Cilantro ( Coriandrum sativum L.) Plants Grown in Organic Soil. J. Agric. Food Chem. 61, 6224-6230 (2013).

30. Zhang, Z. et al. Uptake and distribution of ceria nanoparticles in cucumber plants. Metallomics 3, 816 (2011).

31. Wang, G. et al. Influence of phosphate on phytotoxicity of ceria nanoparticles in an agar medium. Environ. Pollut. 224, 392-399 (2017).

32. Wason, M. S. \& Zhao, J. Cerium oxide nanoparticles: potential applications for cancer and other diseases. Am. J. Transl. Res. 5, 126-31 (2013).

33. Pelletier, D. A. et al. Effects of Engineered Cerium Oxide Nanoparticles on Bacterial Growth and Viability. Appl. Environ. Microbiol. 76, 7981-7989 (2010).

34. Chen, H.-I. \& Chang, H.-Y. Synthesis of nanocrystalline cerium oxide particles by the precipitation method. Ceram. Int. 31, 795-802 (2005).

35. Kulthong, K., Srisung, S., Boonpavanitchakul, K., Kangwansupamonkon, W. \& Maniratanachote, R. Determination of silver nanoparticle release from antibacterial fabrics into artificial sweat. Part. Fibre Toxicol. 7, 8 (2010).

36. Barker, P. J. \& Branch, A. The interaction of modern sunscreen formulations with surface coatings. Prog. Org. Coatings 62, 313-320 (2008).

37. Salter, C., Langhus, D. L. \& Kauffman, G. B. Chemistry for Everyone The Chemistry of Swimming Pool Maintenance W Products of Chemistry edited by. Journal of Chemical Education 84, (2007).

38. Bhattacharjee, S. DLS and zeta potential - What they are and what they are not? $J$. Control. Release 235, 337-351 (2016).

39. Buettner, K. M., Rinciog, C. I. \& Mylon, S. E. Aggregation kinetics of cerium oxide nanoparticles in monovalent and divalent electrolytes. Colloids Surfaces A Physicochem. Eng. Asp. 366, 74-79 (2010).

40. Wang, C. et al. Quantitative measurement of aggregation kinetics process of nanoparticles using nanoparticle tracking analysis and dynamic light scattering. $J$. Nanoparticle Res. 21, 87 (2019).

41. Hou, J. et al. Nanoparticle tracking analysis versus dynamic light scattering: Case study on the effect of $\mathrm{Ca} 2+$ and alginate on the aggregation of cerium oxide nanoparticles. J. Hazard. Mater. 360, 319-328 (2018).

42. Nimesh, S., Chan, R. \& Gupta, N. Advances in nanomedicine for the delivery of 
therapeutic nucleic acids.

43. Wiesner, M. R. \& Plata, D. L. Incinerator filters nanoparticles. Nat. Nanotechnol. 7, 487-488 (2012).

44. Schwabe, F. et al. Dissolution and transformation of cerium oxide nanoparticles in plant growth media. J. Nanoparticle Res. 16, 2668 (2014).

45. Exo Terra : Sunray fixture / Metal Halide Fixture with Ballast and Bulb. Available at: http://www.exo-terra.com/en/products/sunray_fixture.php. (Accessed: 24th April 2018)

46. He, D. et al. Microwave-assisted rapid synthesis of $\mathrm{CeO} 2$ nanoparticles and its desulfurization processes for $\mathrm{CH} 3 \mathrm{SH}$ catalytic decomposition. Chem. Eng. J. 289, 161-169 (2016).

47. Bastien, N., Millau, J.-F., Rouabhia, M., Davies, R. J. H. \& Drouin, R. The sunscreen agent 2-phenylbenzimidazole-5-sulfonic acid photosensitizes the formation of oxidized guanines in cellulo after UV-A or UV-B exposure. J. Invest. Dermatol. 130, 2463-71 (2010).

48. Xia, T. et al. Comparison of the Mechanism of Toxicity of Zinc Oxide and Cerium Oxide Nanoparticles Based on Dissolution and Oxidative Stress Properties. ACS Nano 2, 2121-2134 (2008).

49. Heckert, E. G., Karakoti, A. S., Seal, S. \& Self, W. T. The role of cerium redox state in the SOD mimetic activity of nanoceria. Biomaterials 29, 2705-2709 (2008).

50. Inerbaev, T. M. et al. Aqueous medium induced optical transitions in cerium oxide nanoparticles. Phys. Chem. Chem. Phys. 17, 6217-6221 (2015).

51. Bêche, E., Charvin, P., Perarnau, D., Abanades, S. \& Flamant, G. Ce 3d XPS investigation of cerium oxides and mixed cerium oxide (CexTiyOz). Surf. Interface Anal. 40, 264-267 (2008).

52. Gupta, A., Das, S., Neal, C. J. \& Seal, S. Controlling the surface chemistry of cerium oxide nanoparticles for biological applications. J. Mater. Chem. B 4, 3195$3202(2016)$.

53. Gogos, A., Wielinski, J., Voegelin, A., Emerich, H. \& Kaegi, R. Transformation of cerium dioxide nanoparticles during sewage sludge incineration. Environ. Sci. Nano 6, 1765-1776 (2019).

54. Rajasulochana, P. \& Preethy, V. Comparison on efficiency of various techniques in treatment of waste and sewage water - A comprehensive review. Resour. Technol. 2, 175-184 (2016). 
55. Schymura, S., Fricke, T., Hildebrand, H. \& Franke, K. Elucidating the Role of Dissolution in $\mathrm{CeO} 2$ Nanoparticle Plant Uptake by Smart Radiolabeling. Angew. Chemie - Int. Ed. 56, 7411-7414 (2017).

56. Pulido-Reyes, G. et al. Untangling the biological effects of cerium oxide nanoparticles: the role of surface valence states. Sci. Rep. 5, 15613 (2015). 


\subsection{Supporting Information}

Table S5-1: The test results for the content of raw wastewater

\begin{tabular}{|c|c|c|}
\hline Test & Units & Sample \\
\hline $\begin{array}{c}\text { Chemical Oxygen Demand, } \\
\text { COD }\end{array}$ & $\mathrm{mg} / \mathrm{L}$ & 407 \\
\hline Total Nitrogen, N & $\mathrm{mg} / \mathrm{L} \mathrm{N}^{\mathrm{m}}$ & 31 \\
\hline Total Phosphorus, $\mathrm{P}$ & $\mathrm{mg} / \mathrm{L} \mathrm{PO}_{4}{ }^{3-}$ & 2.2 \\
\hline Total Sulfate, S & $\mathrm{mg} / \mathrm{L} \mathrm{SO}_{4}{ }^{-}$ & 98 \\
\hline Ammonia, NH${ }_{3}-\mathrm{N}$ & $\mathrm{mg} / \mathrm{L} \mathrm{NH}_{3}-\mathrm{N}$ & 28 \\
\hline Nitrate, NO $3-\mathrm{N}$ & $\mathrm{mg} / \mathrm{L} \mathrm{NO}_{3}{ }^{-}-\mathrm{N}$ & 0.7 \\
\hline Turbidity & $\mathrm{NTU}$ & 123 \\
\hline Absorbance @254nm & - & 1.3303 \\
\hline Total Solids & $\mathrm{mg} / \mathrm{L}$ & 907 \\
\hline Total Volatile Solids & $\mathrm{mg} / \mathrm{L}$ & 326 \\
\hline
\end{tabular}


Table $\mathrm{S}$ 5-2: TEM Images and EDS peaks of $\mathrm{CeO}_{2}$ nanoparticles extracted from sunscreen A and sunscreen B after 4-weeks of incubation by incineration, solvent extraction and water extraction.

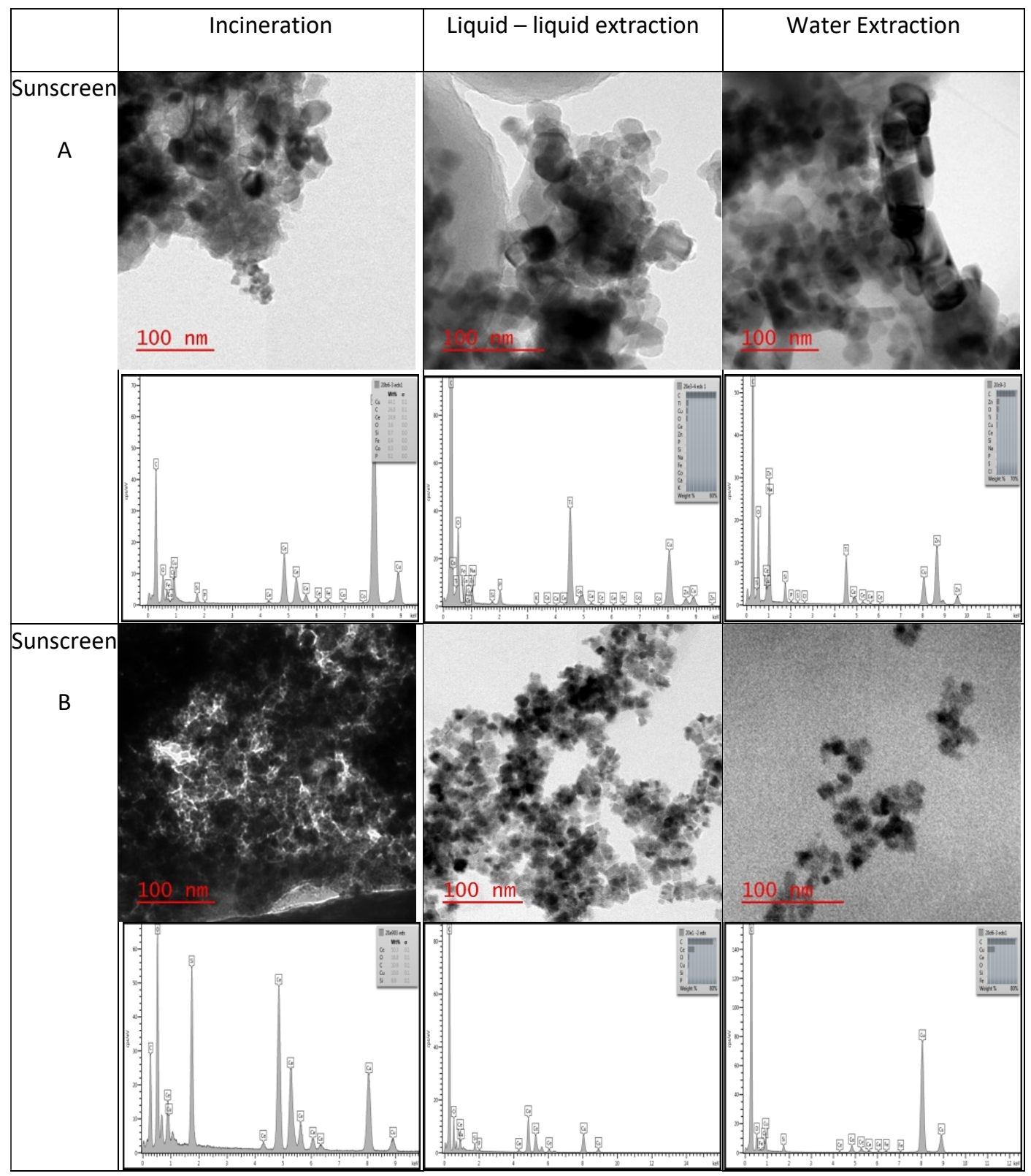



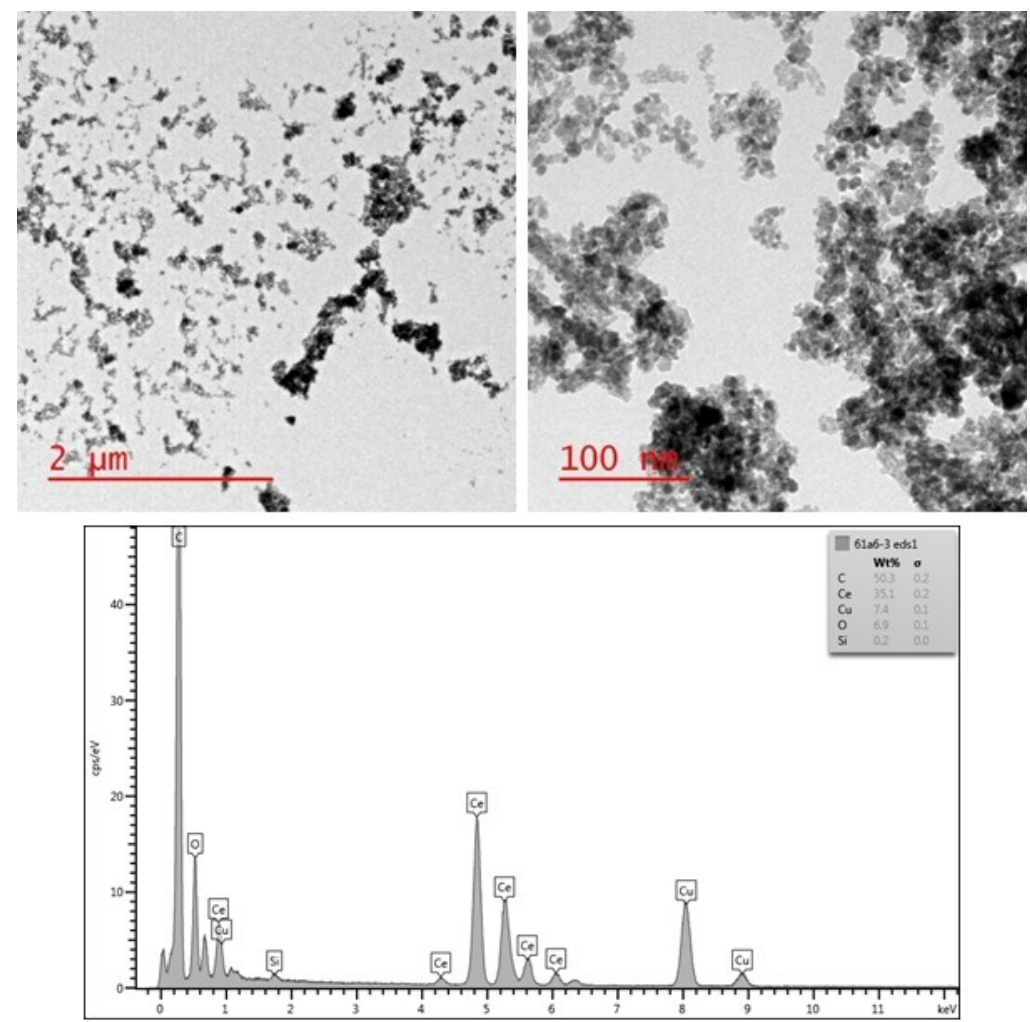

Figure S 5-1: Cerium oxide nanoparticles imaged from the TEM. The EDS reveals the pure cerium oxide. 


\section{Chapter 6: Concluding Remarks}

\subsection{Conclusion}

The ever-increasing use of nanoparticles in commercial products will lead to rising nanoparticle levels in the environment ${ }^{1}$. Although the environmental effects of pristine nanoparticles have been extensively researched in the past few decades, the final form of nanoparticles entering the environment has remained relatively unexplored ${ }^{2-4}$. Commercial products are exposed to various physical and chemical stimuli throughout their lifecycle that will transform the nanoparticles contained in the product. This study investigates the morphological and compositional transformations of $\mathrm{Ag}, \mathrm{ZnO}, \mathrm{CeO}_{2}$ nanoparticles in commercial products as they are put through day-to-day use scenarios followed by the leaching of these nanoparticles into the wastewater collection stream on its way to the treatment facility.

AgNPs are applied to commercial products, mainly textiles, for its antimicrobial properties $^{5}$. In order to mimic the life cycle of the product, pristine nanoparticles and nanoparticle-containing fabrics were exposed to sweat, washed with bleach and detergent, and incubated in wastewater. The weathering resulted in the nanoparticles aggregating and agglomerating. Moreover, the likely formation of $\mathrm{AgCl}$ and $\mathrm{Ag}_{2} \mathrm{~S}$ was observed.

ZnO NPs can absorb light in the UV range as well as being antimicrobial, like AgNPs. Pristine ZnO NPs, along with T-shirt, sunscreen, socks, and baby powder containing ZnO NPs, were exposed to sweat, UV light, chlorinated pool water, as well as washing with bleach and detergent, and incubation in wastewater. The extensive 
dissolution of $\mathrm{ZnO}$ NPs accelerated by a low $\mathrm{pH}$ was observed. Observation of prevalent agglomeration was attributed to the clustered precipitation of $\mathrm{ZnO}$ after dissolution. The likely transformation into $\mathrm{ZnCl}_{2}$ and $\mathrm{ZnS}$ was observed.

$\mathrm{CeO}_{2} \mathrm{NPs}$ are effective in absorbing UV light, as well as being an antioxidant and a radical scavenger. In order to explore new commercial applications, experiments were focused on commercial products that will utilize the UV absorbing property of $\mathrm{CeO}_{2} \mathrm{NPs}$. Pristine $\mathrm{CeO}_{2} \mathrm{NPs}$ and sunscreens containing $\mathrm{CeO}_{2} \mathrm{NPs}$ were exposed to sweat, chlorinated pool water, UV light, and then incubated in wastewater. $\mathrm{CeO}_{2} \mathrm{NPs}$ contain cerium at a +3 and +4 oxidation state in a constant ratio. After weathering the nanoparticles, there was a higher percentage of $\mathrm{Ce}^{3+}$ in the nanoparticles. Furthermore, the nanoparticles were found to aggregate but not agglomerate. The possible formation of $\mathrm{CeCl}_{3}$ and $\mathrm{Ce}_{2} \mathrm{~S}_{3}$ was observed.

Despite the dissolution and transformations of the nanoparticles found in this study, they are not completely dissolved as they make their way through the wastewater collection system. Aggregation of the nanoparticles, as well as association with chlorine and sulfur, had been found to be a common transformation of the nanoparticles. It was also noted that the nanoparticles exhibited the most drastic transformation when incubated in wastewater. The transformation exhibited by pristine nanoparticles incubated in wastewater alone was identical to the pristine and commercial sourced nanoparticles that had been put through the simulated day-to-day use followed by wastewater incubation. 


\subsection{Limitations and Applications of this Research}

Despite observing multiple transformations that take place on the nanoparticles, some transformations that take place in the nanoparticles could have been undetected due to limitations of the methods used. TEM samples of leached nanoparticles were prepared from solution, which avoids the analysis of precipitates like $\mathrm{AgCl}$ that could have formed. Moreover, additional analysis techniques like X-ray absorption near edge structure (XANES) could have been used to determine the concentration of each transformed chemical species like $\mathrm{ZnCl}_{2}$ and $\mathrm{ZnS}$. In order to better characterize the extent of nanoparticle aggregation and agglomeration, atomic force microscopy (AFM) could provide a three-dimensional image of the distribution and size of nanoparticles.

The morphological and compositional transformations of the nanoparticles found in this study could alter the effects the nanoparticles have on the environment when compared to their pristine form. Using weathered nanoparticles in environmental studies would be best. Moreover, knowing the transformed form of nanoparticles entering the wastewater treatment facility will help in designing new treatment processes. Instead of having to simulate the life cycle of the product to achieve accurately weathered nanoparticles, this research had shown that 24-hours of incubation with sewage water alone is likely sufficient to prepare a relevant weathered sample. Despite the attempts to artificially simulate and weather possible life-cycle scenarios, simulating a natural lifecycle for nanoparticles in commercial products is impossible due to the seemingly infinite paths the nanoparticle can take through its journey from synthesis, product incorporation, product use case, disposal and finally leaching into the environment. However, applying 
the proposed weathering model will provide a more accurate understanding of the environmental effects of nanoparticles and aid in future decision making.

\subsection{Future work recommendations}

In the future, further transformations of the nanoparticles beyond the wastewater incubation will be studied. The nanoparticles will be weathered by wastewater and biosolid treatment scenarios. Moreover, in the pursuit to thoroughly understand nanoparticle transformation, $\mathrm{Ag}, \mathrm{ZnO}$, and $\mathrm{CeO}_{2} \mathrm{NPs}$ with various surface coatings as well as other metal and metal oxide nanoparticles such as $\mathrm{CuO}, \mathrm{TiO}_{2}, \mathrm{Au}$, and $\mathrm{Fe}_{2} \mathrm{O}_{3}$ nanoparticles will be exposed to respective relevant product life-cycle scenarios ${ }^{6}$. Furthermore, as the industrial use of carbon-based nanoparticles, polymeric nanoparticles, quantum dots, and nanoclays, as well as other advanced materials like aptamers and supramolecules, are becoming more prevalent ${ }^{7}$, their possible transformations due to its life cycle will be explored. Based on the acquired knowledge from this thesis, it can be deduced that the transformation during the life-cycle of these

advanced materials will be imminent but the type and extent of transformations will vary between each material. 


\subsection{References}

1. Keller, A. A. \& Lazareva, A. Predicted Releases of Engineered Nanomaterials: From Global to Regional to Local. Environ. Sci. Technol. Lett. 1, 65-70 (2014).

2. Pulido-Reyes, G. et al. Untangling the biological effects of cerium oxide nanoparticles: the role of surface valence states. Sci. Rep. 5, 15613 (2015).

3. Nel, A., Xia, T., Mädler, L. \& Li, N. Toxic potential of materials at the nanolevel. Science 311, 622-7 (2006).

4. Manzo, S. et al. Investigation of ZnO nanoparticles' ecotoxicological effects towards different soil organisms. Environ. Sci. Pollut. Res. 18, 756-763 (2011).

5. Kim, D. et al. Selective Killing of Pathogenic Bacteria by Antimicrobial Silver Nanoparticle_-Cell Wall Binding Domain Conjugates. ACS Appl. Mater. Interfaces 10, 13317-13324 (2018).

6. Tourinho, P. S. et al. Metal-based nanoparticles in soil: Fate, behavior, and effects on soil invertebrates. Environ. Toxicol. Chem. 31, 1679-1692 (2012).

7. Santos, C. S. C. et al. Industrial Applications of Nanoparticles - A Prospective Overview. Mater. Today Proc. 2, 456-465 (2015). 